\title{
ZEITSCHRIFT FÜR INTERPRETATIVE SCHUL- UND UNTERRICHTSFORSCHUNG
}
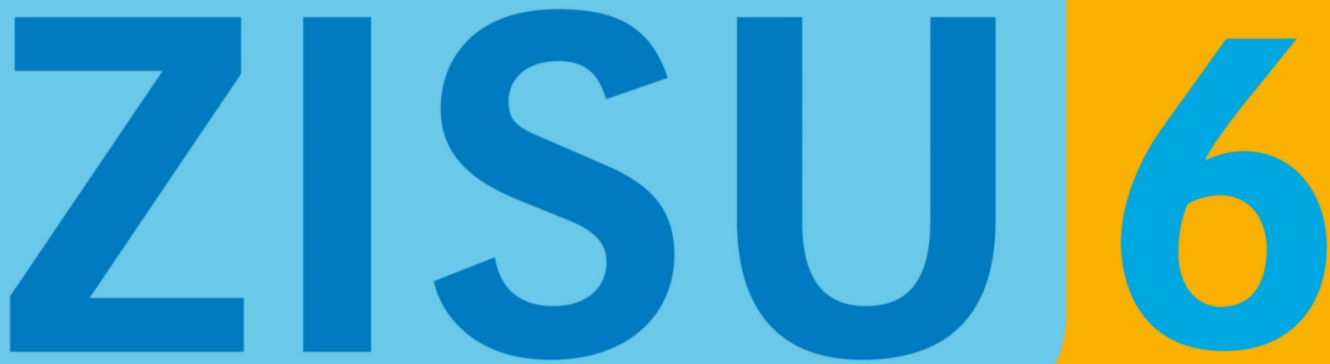

Empirische Beiträge

aus Erziehungswissenschaft

und Fachdidaktik

\section{Thema: Performativität und Medialität im Unterricht}




\section{Inhalt}

\section{ZISU}

\section{Zeitschrift für interpretative Schul- und Unterrichtsforschung}

\section{Editorial}

Matthias Proske,

Medialität und Performativität im Unterricht:

Anne Niessen

Zwischen Hervorbringen und Übertragen, Inszenieren und Wahrnehmbarmachen schulischen Wissens, Könnens und Sollens. Eine Einführung

\section{Thementeil}

Johanna Ahlrichs, Felicitas Macgilchrist

Maria Hallitzky, Beate Beyer, Christopher Hempel, Johanna Leicht, Ellen Schroeter

Ute Bender, Edina Krompak

Regula Fankhauser, Angela Kaspar

May Jehle
Medialität im Geschichtsunterricht: Die Rolle des Schulbuchs beim Vollzug von ,Geschichte“

,Das Märchen von dem Machandelbaum“ oder wie Unterrichtsstoff durch Medien inszeniert wird

Essbare Dinge in Lehr- und Lernprozessen - Sprachförderung mit Lebensmitteln im Kindergartenalltag

Der bewegte Körper im Unterricht: zwischen medialer Inszenierung und Manifestation des Leiblichen

Möglichkeitsräume des Performativen. Potenziale handlungsorientierter Methoden zur Förderung von Mündigkeit im Politikunterricht Subjektpositionen im Spannungsfeld zwischen Aufgabenstellung und Peers

Zwischen Mitgliedschaft und Teilhabe. Praxeologische

Thorsten Merl Rekonstruktionen von Teilhabeformen im inklusiven Unterricht 


\section{Rezensionen}

Till-Sebastian Idel

Alkemeyer, Thomas/Kalthoff, Herbert/Rieger-Ladich, Markus (2015). Bildungspraxis. Körper, Räume, Objekte. Weilerswist: Velbrück Wissenschaft. 


\section{Editorial}

Matthias Proske und Anne Niessen

\section{Medialität und Performativität im Unterricht: Zwischen Hervorbringen und Übertragen, Inszenieren und Wahrnehmbarmachen schulischen Wissens, Könnens und Sollens}

\section{Eine Einführung}

Eine Herausforderung für schulische Vermittlungsprozesse besteht darin, dass das, was Schülerinnen und Schüler sich aneignen sollen, im Unterricht zunächst erst einmal sichtbar, hörbar und fassbar (gemacht) werden muss. Die Wahrnehmung des zu Vermittelnden im Modus sinnbasierter Erlebnisse und Erfahrungen ist in unterschiedlicher Weise die Voraussetzung dafür, dass Kenntnisse erworben, Fertigkeiten habitualisiert und Haltungen ausgebildet werden können, kurzum dass Lernen oder Bildung überhaupt stattfinden kann.

\section{Schulische Vermittlung und die Frage nach den sozio-materiellen Bedingungen von Unterricht}

Auf die Herausforderung, dass die zu behandelnden ,Sachen' des Unterrichts nicht einfach ,da sind' bzw. vorliegen, hat die didaktische Tradition mit dem Begriff der „Artikulation“ reagiert (vgl. Prange 2005) und damit die soziale, sachliche und zeitliche Gliederung des Unterrichtsganges zum Zwecke der Synchronisation von Unterrichten und Lernen gemeint. Unübersehbar ist jedoch, dass im didaktischen Diskurs die medialen und körperlich-inszenatorischen Implikationen des schulischen Vermittlungsgeschehens in systematischer Hinsicht entweder keine oder nur eine äußert nachgeordnete Rolle spielen.

Dies trifft auch auf Pranges Konzeptualisierung einer auf der Zeigegebärde aufruhenden operativen Pädagogik zu. Zwar liefern sowohl der Rückgriff auf die körperliche Fundierung und den performativen Vollzug des Zeigens (Prange 2005: 67ff.) als auch dessen Zweckbestimmung, ,Mensch und Welt für die Lernenden zur Erscheinung zu bringen“" (Prange/Strobel-Eisele 2006: 47), Anschlussstellen für eine systematische Klärung der Bedeutung von körperlichen Vollzügen einerseits und von medialen Repräsentationen der thematischen Bezugspunkte pädagogischer Vermittlung andererseits. Diese werden in (fach-)didaktischen Diskursen jedoch nur selten aufgegriffen. Es dominiert eine Sichtweise, in der die Darstellungsproblematik meist an die Mediendidaktik delegiert und auf die Frage reduziert wird, welche mediale Repräsentation als didaktisch angemessen im Hinblick auf Situation, Ziele, Lernvoraussetzungen, Inhalte und organisatorische Bedingungen des jeweiligen Unterrichtsarrangements gelten kann (vgl. Tulodziecki/Herzig/Blömeke 2017: 187f.). ${ }^{1}$ Auch für die Körperlichkeit des Vollzugs

1 Diese Beschränkung verwundert umso mehr, als bereits im pädagogisch-erziehungswissenschaftlichen Diskurs, etwa mit dem Erfahrungsbegriff (vgl. dazu Bollnow 1968; Prange 
von Unterricht und der Hervorbringung von Wissen, Können und Sollen hat der (fach-) didaktische Diskurs so gut wie keine Begrifflichkeit entwickelt. ${ }^{2}$

In der qualitativen Unterrichtsforschung ist dagegen in den letzten Jahren ein deutlich erkennbares Interesse an den sozio-materiellen Voraussetzungen und Bedingungen der Hervorbringung von Wissen, Können und Sollen zu beobachten (vgl. als Überblick: Rabenstein 2017 sowie Hackl/Stifter 2017). In den Forschungsfokus gerückt ist dabei neben der Räumlichkeit unterrichtlicher Arrangements (z. B. Breidenstein 2004; Putzier 2012; Hackl 2015; Rißler 2015) und der Materialität schulischer Wissensobjekte (z. B. Fetzer 2012, 2015; Rabenstein/Wienike 2012; Röhl 2013; Martens/Asbrand/ Spieß 2015) insbesondere die körper- und kommunikationsgebundene Hervorbringung und die mediale Repräsentation schulischen Wissens und Könnens (z. B. Alkemeyer/ Pille 2016; Greiffenhagen 2015; Macgilchrist 2017; Putzier 2011). Körper- und kommunikationsgebundene Hervorbringung und Medialisierung sind die beiden Aspekte einer sozio-materiellen Perspektive auf Unterricht, an die wir mit dem vorliegenden Schwerpunktthema der ZISU anschließen. Dabei soll es in grundlegender Weise um die Frage der pädagogischen Inszenierung dessen gehen, was in Schule vermittelt und gelernt wird.

\section{Hervorbringen und Übertragen, Inszenieren und Wahrnehmbarmachen: Der Beitrag der Theorieangebote Performativität und Medialität}

Um die sozialen und kulturellen Praxen der pädagogischen Inszenierung von Wissen, Können und Sollen sowie deren Voraussetzungen beschreib- und analysierbar zu machen, bieten sich die Theorieangebote der Performativität und Medialität an, insofern ihr gemeinsamer Fluchtpunkt darin besteht, zunächst das Konstitutions- und Hervorbringungsvermögen von Körpern, Dingen und symbolischen Zeichen in solchen Praxen zu betonen (Krämer 2004: 19). Diese Betonung zeige sich - zumindest dann, wenn man in medientheoretischer Hinsicht einer generativistischen Position folgt, die die Prägekraft des Mediums gegenüber ihren Botschaften hervorhebt -, ,,in der Privilegierung des Machens im Darstellen, des Erzeugens im Widerfahren“ (ebd.: 23, Herv. d. Hrsg.). Die generativistische Prämisse im Konzept der Medialität, dass Repräsentationen immer strukturiert werden durch die genutzten Medien, ist nicht nur im Zentrum der meisten gegenwärtigen medientheoretischen Ansätze zu finden. Sie verweist auch auf das jedem Medium eigentümliche Set von Eigenschaften, die wiederum aus dem Zusammen-

1989; Hollstein 2011) oder dem Vermittlungsbegriff (Kade 1997; Gruschka 2002), durchaus begrifflich-systematische Arbeiten zur Repräsentationsproblematik existieren, an die angeschlossen werden könnte.

2 Als Ausnahme ließe sich auf die Überlegung Gottfried Hausmanns (1959) verweisen, der die didaktische Gestaltung des Unterrichts als dramaturgische Inszenierung in Analogie zum Theaterspiel beschreibt. 
hang von technisch-materiellen Merkmalen und kulturellem Gebrauch resultieren (vgl. Hickethier 2010). Zu denken ist dabei an die Eigenschaften der grundlegenden Medialitäten der Oralität und Literalität ${ }^{3}$ sowie der Audiovisualität, wobei sich in letzterer am deutlichsten die Technisierung von Kommunikation zeigt.

Vor allem auf die Arbeiten Erika Fischer-Lichtes (1998a, 1998b, 2004a, 2004b) ist zurückzuführen, dass die mediale Trias von Oralität, Literalität und Audiovisualität inzwischen um das Konzept der Theatralität bzw. Performativität ergänzt gedacht wird (vgl. Hickethier 2010: 27f.). Das Konzept der Performativität stammt ursprünglich aus der Sprachphilosophie, wurde dann - inspiriert von der Besonderheit künstlerischer Inszenierung und Aufführung - kulturwissenschaftlich weiterentwickelt (vgl. als Überblick: Wirth 2002b) und wird inzwischen auch in den Fachwissenschaften und im erziehungswissenschaftlichen Kontext als sensibilisierendes Konzept breiter rezipiert (vgl. z. B. Wulf/Zirfas 2007). Während Wulf und Zirfas einen ,performative turn in den Kultur- und Sozialwissenschaften" ausmachen und dessen Auswirkungen auf die Erziehungswissenschaft zu erschließen versuchen (Wulf/Zirfas 2007: 7), ordnet Werner Helsper erziehungswissenschaftliche „Studien zu ,Kulturen des Performativen““ in den Kontext des cultural turn in der Schultheorie ein: „Die Besonderheit dieses Ansatzes beruht darauf, dass die performative alltägliche Gestaltung der Schule als Inszenierung und Darstellung in schulischen Ritualen und körperlichen Praktiken im Zentrum steht.“ (Helsper 2008: 65) Auch wenn das Konzept der Performativität in der Unterrichtsforschung über keine lange Tradition verfügt, lassen sich doch in Bezug auf seine Verwendung (mindestens) zwei Ebenen unterscheiden: a) Performativität als Merkmal einzelner Unterrichtssituationen, also beispielsweise eines Rollenspiels oder eines - auch musikalischen - Vortrags, und b) Performativität als übergeordnete Perspektive auf den Inszenierungszusammenhang Unterricht.

Performativität ist stark durch Referentialität geprägt, weil sie Bezug nimmt auf die vorhandene ,praktische, soziale und kulturelle Ordnung von Phänomenen“, „ihre Prozesse, Modalitäten, Logiken und Funktionen“ (Wulf/Zirfas 2007: 16). Vier Aspekte lassen sich an dem Konzept unterscheiden: Erstens bezieht sich Performativität auf performance als „,Vorgang einer Darstellung durch Körper und Stimme vor körperlich anwesenden Zuschauern“, zweitens auf Inszenierung als „spezifische[m] Modus der Zeichenverwendung in der Produktion“, drittens auf Körperlichkeit als Resultat der „Darstellung bzw. des Materials“ und viertens auf Wahrnehmung im Hinblick ,auf den Zuschauer, seine Beobachtungsfunktion und -perspektive“ (Wirth 2002a: 38f. mit Bezug auf Fischer-Lichte). Mit diesen Unterscheidungen wird deutlich, dass Fischer-Lichte Performativität sowohl semiotisch im Sinne symbolischer Zeichennutzungen versteht, gleichzeitig den Begriff aber auch nutzt, um über die Aspekte der Inszenierung, der Körperlichkeit und der Wahrnehmung die kulturerzeugende Funktion von Praxen und Ereignissen zu unterstreichen (vgl. Fischer-Lichte 1998a: 86f.; Wirth 2002a: 38f.). Jeder medialen Darstellung und Hervorbringung von Phänomenen des Wissens, Könnens und Sollens sind in diesem Sinne performative Effekte inhärent.

3 Dabei ist unstrittig, dass auch mündliche Praktiken wie etwa der Vortrag oder die Präsentation konzeptuell durch schriftliche strukturiert sein können. Und umgekehrt zeigt der Gebrauch einer Reihe neuer Kommunikationsmedien, dass schriftliche Praktiken beeinflusst werden durch solche alltäglichen Sprechens. 
Als ein Schlüssel zur Verknüpfung der beiden Konzepte Medialität und Performativität wird der Begriff der „Aisthetisierung“ angesehen, der sowohl den Vollzug von Praktiken und Ereignissen als auch deren Wahrnehmung bezeichnen soll (Krämer 2004: 21). Ereignisse sind in dieser Perspektive ,in Szene gesetztes Geschehen“ (ebd.: 14), die nicht nur in bestimmter medial verkörperter Weise etwas zeigen, sondern deren Bedeutung durch die eigensinnigen Wahrnehmungen der unterschiedlichen Beteiligten strukturiert wird.

Versucht man die Einsätze der jüngeren kultur- und medientheoretischen Diskussion über Medialität und Performativität zuzuspitzen, dann scheint in unseren Augen das wesentliche Merkmal der Verknüpfung beider Konzepte darin zu bestehen, das ihnen inhärente generativistische Theorem, Wirklichkeit immer als im operativen Vollzug hervorgebrachte zu verstehen, nicht einseitig gegen die „Mittlerfunktion“ von Medien auszuspielen. Diese besteht vor allem darin, ,das ,Sozialpotenzial des Mediums‘ gegenüber seinem ,Technikpotenzial' in den Vordergrund“" zu rücken, d. h. das Vermögen, als Medium soziale Relationen über Differenzen hinweg zu stiften (Krämer 2008: 115). Mit dem Gebrauch des Mittlerbegriffes werden dann Übertragung und Dissemination, Wahrnehmbarmachen und Erscheinenlassen als wesentliche Leistungen von Medien mitdenkbar (vgl. ebd., insb.: 32-36). Diese anti-demiurgische Rehabilitierung des „postalischen Prinzips der Kommunikation“ (ebd.: 12ff.) will nicht unterkomplexen Formen der Widerspiegelung oder Abbildung von Wirklichkeit durch Medien das Wort reden $^{4}$, sondern darauf aufmerksam machen, dass es in sozial eingebetteten Übertragungsprozessen angesichts von Kontingenz und Unzugänglichkeit immer um das Offenbarmachen und die Artikulation von Differenzen geht (ebd.: 264ff).

\section{Das Potential der Konzepte Performativität und Medialität für die qualitative Unterrichtsforschung}

Was lässt sich für Unterrichtsforschung gewinnen, wenn man die hier skizzierten theoretischen Gehalte der Konstrukte Medialität und Performativität nutzt? Insbesondere die differenztheoretische Wendung im Verständnis von Übertragung und Repräsentation bzw. Hervorbringung und Inszenierung macht den neueren Diskurs zu Medialität und Performativität unseres Erachtens zu einer interessanten Referenz für die erziehungswissenschaftliche wie fachdidaktische Unterrichtsforschung.

Diese Fokussierung erlaubt es, Unterrichtsmedien und deren Medialitäten auf ihre wechselseitigen, aber kontingenten Referenzen zu beobachten, z. B. im Verhältnis von Demonstrationsexperiment, darüber geführtem Unterrichtsgespräch sowie darauf bezogenen Tafelanschrieben und Hefteinträgen. Untersuchbar wird damit die Frage, welches Wissen entlang der Differenzlinien von disziplinär-fachlicher, unterrichtlicher und alltäglicher epistemischer Ordnung im Unterricht in welcher Weise wahrnehmbar gemacht wird, etwa wenn über die Wandtafel das Unterrichtsgespräch oder ein Demonstrations-

4 Vgl. für die Diskussion um den Begriff der Repräsentation aus der Perspektive einer historischen Epistemologie z. B. Rheinberger et al. (1997), aus philosophischer Perspektive z. B. Mormann (1997). 
experiment in bestimmter Weise kommentiert und vereindeutigt wird, dabei aber die Medien dieser Kommentierung und Vereindeutigung als Medien selbst hinter ihren Effekten verschwinden.

Der differenztheoretische Blick auf Hervorbringungen lenkt zudem die Aufmerksamkeit auf die Frage, wie unterrichtliche Ereignisse in Szene gesetzt werden bzw. sich in Szene setzen, damit Abwesendes und Unzugängliches sich dennoch zeigen kann und vergegenwärtigt wird. Betrachtet man Unterricht unter dem Vorzeichen der ,wirklichkeitskonstituierenden Kräfte" medial-performativen Handelns (Forster 2007: 225) relativiert dies die didaktische Intentionalität der Repräsentation, mit der das als ,eigentlich wichtig' markiert, was durch die unterrichtliche Inszenierung vermittelt werden soll. In den Vordergrund rückt stärker der jeweilige operative Vollzug des Unterrichts, in dem die Gegenwärtigkeit und Präsenz des ,Aufführens` von Wissen, Können und Sollen ihre eigenen Wirkungen entfaltet.

Versteht man Unterricht als kommunikativ-materielle Ordnung des Pädagogischen, dann scheint den Konzepten der Performativität und Medialität eine hohe theoretische Produktivität zuzukommen. Sie ermöglichen es der Unterrichtsforschung, Praxen des Unterrichtens zwischen der Hervorbringung und der Übertragung, der Inszenierung und der Wahrnehmbarmachung schulischen Wissens, Könnens und Sollens zu untersuchen.

\section{Beiträge zum Heftthema}

Johanna Ahlrichs und Felicitas Macgilchrist zeigen in ihrem ethnographisch angelegten Beitrag Medialität im Geschichtsunterricht: Die Rolle des Schulbuchs beim Vollzug von ,Geschichte', wie die medialen Eigenschaften des gedruckten Schulbuchs (als Leseobjekt mit bestimmten graphischen Strukturmerkmalen) eher beiläufig - die Autorinnen sprechen hier von den „kleinen Praktiken“ im alltäglichen Unterricht - spezifische Verhaltensweisen des Umgangs mit ihm konstituieren. Mit dieser Fokussierung auf das Zusammenspiel von Medialität und Performativität, von technisch-materiellen Merkmalen und sozio-kulturellem Gebrauch wird das, so die zentrale These des Beitrages, was in der Schule unter ,Geschichte' verstanden wird, wahrnehmbar gemacht und in diesem Sinne hervorgebracht. Die ethnographische Rekonstruktion zeigt, dass für dieses schulische Wahrnehmbarmachen von Geschichte das wahrheitserzeugende „Vergegenständlichen“ und das dekontextualisierende „Segmentieren“ eine zentrale Rolle spielen. Gegen eine rein formale Deutung dieser Befunde insistieren die Autorinnen zudem darauf, dass solche Medienpraktiken eine inhaltliche und insofern auch politische Wirkkraft haben: Zum einen in Bezug auf die Relevanzsetzung bestimmter Geschichten mit dabei vollzogenen In- und Exklusionen, zum anderen breiter in Bezug auf die Modalitäten des Denkbaren sowie Sicht- und Sagbaren über historisch grundlegende Phänomene von Autorität und sozialer Ordnung.

In ihrem Beitrag ,Das Märchen von dem Machandelbaum 'oder wie Unterrichtsstoff durch Medien inszeniert wird schließen Maria Hallitzky, Beate Beyer, Christopher Hempel, Johanna Leicht und Ellen Schroeter unmittelbar an die oben skizzierte Doppelperspektive der Konzepte Medialität und Performativität an, wenn sie in ihrer vi- 
deobasierten und sequenzanalytisch vorgehenden Rekonstruktion des Mediengebrauchs im Literaturunterricht zu Goethes Faust sowohl auf die repräsentative als auch auf die wirklichkeitskonstituierende Bedeutung der eingesetzten Unterrichtsmedien fokussieren. Ihre Analyse zeigt, dass das Verstehensproblem der Schülerinnen im Umgang mit der Komplexität und Mehrdeutigkeit des unterrichtlichen Gegenstandes in der Unterrichtssituation dadurch ,gelöst' wird, dass die Lehrerin die inhaltliche Bearbeitung an ein den Schülerinnen zur Verfügung gestelltes Tablet delegiert. Ähnlich wie Ahlrichs/ Macgilchrist in ihrer Analyse der Bedeutung des Schulbuches im Fach Geschichte interpretieren Hallitzky/Beyer/Hempel/Leicht/Schroeter dieses auf das Tablet bezogene Medienhandeln als Inszenierung einer Autorität des Wissens, von der eine vereindeutigende ,Lösung' des Verstehensproblems erwartet wird. Die Leipziger Forschungsgruppe deutet das Misslingen dieser Delegation als Hinweis auf das ,grundlegende Handlungsproblem von Lehrkräften, die eigene Vermittlungsarbeit zu der in Medien realisierten Vermittlungsarbeit in Beziehung zu setzen“. Es zeige, so die Autorinnen und der Autor weiter, „,dass das Zusammenspiel zwischen personal-medialer und im weitesten Sinn technisch-medialer Vermittlungsarbeit nicht selbstverständlich ist" (Hallitzky et al. i.d.B.).

Sich in einer sozio-materiellen Perspektive verortend untersuchen Ute Bender und Edina Krompak in ihrem Beitrag Essbare Dinge in Lehr- und Lernprozessen - Sprachförderung mit Lebensmitteln im Kindergartenalltag, wie Lebensmittel als ,didaktische Objekte" in Lehr-Lernprozessen verwendet werden. Basierend auf einem ethnographischen Zugang zum Untersuchungsfeld Schweizer Kindergärten werden die erzeugten Feldvignetten mit Hilfe der Grounded-Theory-Methodologie gerade deshalb einer eingehenden Re-Analyse unterzogen, weil die Bezugnahme auf Lebensmittel im Zusammenhang mit dem ursprünglichen Untersuchungsfokus auf unterrichtliche Sprachförderung bei den Beobachterinnen starke Irritationen auslöste. Diese wiederum nehmen die beiden Autorinnen zum Anlass, die „Transformationsleistungen“ durch Lehrende und Lernende bei der Nutzung von Lebensmitteln als didaktische Objekte genauer zu analysieren. Als zentraler Bezugspunkt, aber gleichzeitig auch als Auslöser von Widerständigkeit und Sperrigkeit, - mit Sybille Krämer (s. o.) ließe sich auch formulieren: als Offenbarmachen und Artikulation von Differenzen (2008, S. 264ff) - erweist sich dabei die Essbarkeit der gebrauchten Lebensmittel, die einerseits didaktische Inszenierungen und auch Instrumentalisierungen erlaubt, andererseits aber auch von den pädagogischen Akteuren so beobachtet, behandelt und kontrolliert wird, dass eigensinnige Formen der Bezugnahme (etwa im Genuss der Lebensmittel) die unterrichtliche Ordnung nicht stören (sollen). Dass dies nur zum Teil gelingt, ließe sich auch als Hinweis auf Grenzen didaktischer Objektivierung lesen.

Regula Fankhauser und Angela Kaspar beziehen sich in ihrem Beitrag Der bewegte Körper im Unterricht auf die Konstrukte Medialität und Performativität, indem sie Erika Fischer-Lichtes Reflexion über die Frage aufgreifen, ob der Körper eines Schauspielers auf der Bühne als Medium oder als Leib zu denken sei, nämlich letzterer als ,der gefühlte, empfundene Körper, welcher in seiner Materialität eigengesetzlich auf unser Fühlen, Denken und Handeln einwirkt" (Fankhauser/Kaspar i.d.B.). Fischer-Lichte beschreibt ein Changieren der beiden Betrachtungsweisen (Fischer-Lichte 2004b), was Fankhauser und Kaspar als fruchtbares Motiv für die Analyse der Unterrichtswahrnehmung durch 
Lehrkräfte aufnehmen. Im Rahmen eines qualitativen Forschungsprojekts, in dem Lehrerinnen Videoaufnahmen des eigenen Unterrichts kommentierten, identifizierten die Verfasserinnen Situationen, in denen Lehrerinnen ihren eigenen Körper als Medium verstanden und einsetzten - wenn sich eine Kollegin beispielsweise vornahm, durch die Vermeidung unnötiger Bewegung vor der Klasse eine beruhigende Wirkung auf die Schülerinnen und Schüler auszuüben. Demgegenüber deutete eine andere Lehrkraft die Anzeichen von Übermüdung der Schülerinnen und Schüler als (legitimen) Ausdruck von Leiblichkeit, was bei ihr zusätzlich die Reflexion darüber anregte, welche Zumutungen das Unterrichtsgeschehen für Schülerinnen und Schüler bedeutet und welche Widersprüche sich im alltäglichen Vollzug des Unterrichts in den Manifestationen des Leiblichen spiegeln. Obwohl Fankhauser und Kaspar darauf hinweisen, dass auch die Grundstruktur des Unterrichts immer wieder performativ erzeugt wird, beziehen sie in ihrem Beitrag das Konstrukt Performativität also weniger auf das Unterrichtsgeschehen insgesamt; vielmehr nutzen sie ein Motiv aus der Performativitätstheorie von Erika Fischer-Lichte, um mit seiner Hilfe die Akzentuierungen der Aussagen von Lehrpersonen über ihren eigenen Körper und die Körper der Schülerinnen und Schüler unterrichtstheoretisch fruchtbar zu machen.

Auch May Jehle nutzt in ihrem Beitrag Möglichkeitsräume des Performativen. Potenziale handlungsorientierter Methoden zur Förderung von Mündigkeit im Politikunterricht das Konstrukt Performativität: Anhand der Videoaufnahme eines Rollenspiels zeigt die Verfasserin, dass Schülerinnen und Schüler im Rahmen von Simulationen leibliche Erfahrungen sammeln, die durch die in der Theaterpädagogik beschriebene Erfahrung des ,Dazwischenstehens' geprägt sind. Vor allem interessiert Jehle sich für die dabei möglichen Ambiguitätserfahrungen, die sich in der Spannung zwischen der persönlichen Perspektive und der der Rolle ergeben und denen sie für politische Bildung besondere Bedeutung beimisst: In der Analyse von stills des unterrichtlichen Rollenspiels identifiziert sie emotional aufgeladene Situationen, die neben rationalen Argumenten auch Wertorientierungen sichtbar und damit grundsätzlich bearbeitbar machen. So helfen ihr Elemente aus der Performativitätstheorie, Besonderheiten und Bildungspotentiale simulativer Methoden im Politikunterricht zu erschließen. Dieser und der Beitrag von Fankhauser/Kaspar orientieren sich also an einem Verständnis des Performativen, das an Theorieelemente der Theaterpädagogik anknüpft. Die Autorinnen nutzen Elemente der Reflexion über Performativität, um mit ihrer Hilfe bestimmte Aspekte des Unterrichtsgeschehens (die Perspektive von Lehrenden auf Rolle und Funktion des Körpers im Unterricht und das Bildungspotential von Rollenspielen) hervortreten zu lassen und theoretisch zu erschließen. Auf diese Weise wird eine Möglichkeit der Verbindung von Unterrichtsforschung und der Theorie der Performativität realisiert.

\section{Beiträge des allgemeinen Teils}

Alexandra Flügel nutzt eine anerkennungstheoretische Perspektive für ihre Untersuchung der Organisation der Arbeit am Gruppentisch - in Bezug auf die Subjektpositionen im Spannungsfeld zwischen Aufgabenstellung und Peers. Sie nimmt die Tatsache, dass 
Schülerinnen und Schüler auch an Gruppentischen häufig zur Einzelarbeit aufgefordert sind, zum Anlass, diese Konstellation mit Hilfe videographischer Daten zu erforschen. In der von ihr im Beitrag vorgestellten Szene an einem Vierertisch werden beispielhaft soziale Dynamiken sichtbar, in die Schüler in ihrer Tischgruppe geraten können und die sich deutlich auf die Intensität ihrer Bearbeitung einer Aufgabenstellung auswirken. In Flügels Analyse wird die Problematik deutlich, die aus der Verflechtung peerorientierter und unterrichtsorientierter Anforderungen für Schülerinnen und Schüler erwachsen kann - gerade in der Einzelarbeit am Gruppentisch.

Petra Herzmann und Thorsten Merl betrachten im Kontext einer ethnographischen Feldstudie zu Konzepten inklusiver Schulen die Frage, wie die Anwesenheit von Schülerinnen und Schülern in inklusiven Lernsettings ausgehandelt wird: Zwischen Mitgliedschaft und Teilhabe. Praxeologische Rekonstruktionen von Teilhabeformen im inklusiven Unterricht. Herzmann und Merl unterscheiden dabei eine institutionell gesicherte Mitgliedschaft aller Schülerinnen und Schüler von einer tatsächlichen Teilhabe am Unterricht, die auf einer gemeinsamen Praxis beruht und die - wie sich herausstellt - durch ein zeitweises Verlassen des Klassenraums oder eine Nicht-Beteiligung am Unterrichtsgeschehen nicht grundsätzlich in Frage gestellt wird. Herzmann und Merl identifizieren darüber hinaus Zuschreibungen und Begründungen von Seiten der Lehrenden, die sich auf phasenweise oder partielle Reduktionen unterrichtlicher Anforderungen für einzelne Schülerinnen und Schüler als besondere, aber legitime Form der Teilhabe beziehen. Auf diese Weise treten Aushandlungen der Lehrpersonen untereinander oder mit den Schülerinnen und Schülern sowie Artefakte in Erscheinung, die auf die Besonderheiten der Praxen in inklusiven Lerngruppen bezogen sind.

Markus Hoffmann stellt in seinem Beitrag Deutungsmuster in der Professionsforschung. Anwendung und Ertrag dieses Analyseverfahrens am Beispiel schulischer Sexualerziehung vor. Im Rahmen einer qualitativen Studie führte er Interviews mit Lehrkräften zur schulischen Sexualerziehung. Zum Thema Prostitution stellt er Analysen dreier Interviewausschnitte vor und identifiziert darin zwei biographisch geprägte Deutungsmuster von Lehrkräften, denen zufolge wegen persönlicher Unerfahrenheit bzw. im Hinblick auf eine antizipierte Empfindlichkeit der Schülerinnen und Schüler das Thema Prostitution im Unterricht für problematisch gehalten wird. Laut des dritten erschlossenen Deutungsmusters wird das Thema weniger in einem wertenden als in einem beschreibenden Modus für durchaus unterrichtbar gehalten. Auf diese Weise zeigt Hoffmann die Handlungsrelevanz der rekonstruierten Deutungsmuster auf, was er als Beleg für die Fruchtbarkeit der Analyse von Deutungsmustern in der erziehungswissenschaftlichen Professionsforschung interpretiert. 


\section{Autorenangaben}

\author{
Matthias Proske \\ Professur für Schulforschung mit dem Schwer- \\ punkt Unterrichtstheorien und Schulsystem \\ Department Erziehungs- und Sozialwissen- \\ schaften \\ Humanwissenschaftliche Fakultät \\ Universität zu Köln \\ Innere Kanalstraße 15 \\ D-50823 Köln \\ matthias.proske@uni-koeln.de
}

\author{
Anne Niessen \\ Hochschule für Musik und Tanz Köln \\ Kölner Institut für musikpädagogische \\ Forschung \\ Unter Krahnenbäumen 87 \\ 50668 Köln \\ anne.niessen@hfmt-koeln.de
}

\section{Literatur}

Alkemeyer, Thomas/Pille, Thomas (2016): Bindende Verflechtung. Zur Materialität und Körperlichkeit der Anerkennung im Alltag der Schule. In: Vierteljahresschrift für wissenschaftliche Pädagogik 92, 1, S. 170-194.

Bollnow, Otto Friedrich (1968): Der Erfahrungsbegriff in der Pädagogik. In: Zeitschrift für Pädagogik 14, 3, S. 221-253.

Breidenstein, Georg (2004): KlassenRäume - eine Analyse räumlicher Bedingungen und Effekte des Schülerhandelns. In: Zeitschrift für qualitative Bildungs-, Beratungs- und Sozialforschung 5, 1, S. 87-107.

Fetzer, Marei (2012): Lernen in einer Welt der Dinge. Methodologische Diskussion eines Objektintegrierenden Ansatzes zur mikroethnografischen Unterrichtsanalyse. In: Friebertshäuser, Barbara/Kelle, Helga/Boller, Heike/Bollig, Sabine/Huf, Christina/Langer, Antje/Ott, Marion/ Richter, Sophia (Hrsg.): Feld und Theorie. Herausforderungen erziehungswissenschaftlicher Ethnographie. Opladen: Budrich, S. 121-136.

Fetzer, Marei (2015): Mit Objekten rechnen. Empirische Unterrichtsforschung auf den Spuren von Materialien im Mathematikunterricht. In: Alkemeyer, Thomas/Kalthoff, Herbert/RiegerLadich, Markus (Hrsg.): Bildungspraxis. Körper - Räume - Objekte. Weilerswist: Velbrück Wissenschaft, S. 309-338.

Fischer-Lichte, Erika (1998a): Inszenierung und Theatralität. In: Willems, Herbert/Jurga, Martin (Hrsg.): Inszenierungsgesellschaft. Wiesbaden: Westdeutscher Verlag, S. 81-90.

Fischer-Lichte, Erika (1998b): Grenzgänge und Tauschhandel. Aufdem Weg zu einer performativen Kultur/Verwandlung einer ästhetischen Kategorie. Zur Entwicklung einer neuen Ästhetik des Performativen. In: Dies./Kreuder, Friedemann/Pflug, Isabel (Hrsg.): Theater seit den 60er Jahren, Tübingen/Basel: Franke, S. 1-92.

Fischer-Lichte, Erika (2004a): Ästhetik des Performativen. Frankfurt a.M.: Suhrkamp.

Fischer-Lichte, Erika (2004b): Was verkörpert der Körper des Schauspielers? In: Krämer, Sybille (Hrsg.): Performativität und Medialität. München: Wilhelm Fink, S. 141-162.

Forster, Edgar (2007): Radikale Performativität. In: Wulf, Christoph/Zirfas, Jörg (Hrsg.): Pädagogik des Performativen. Theorien, Methoden, Perspektiven. Weinheim: Beltz, S. 224-237.

Greiffenhagen, Christian (2015): Die Materialität der Mathematik. Wie Mathematik an der Tafel vorgeführt wird. In: Alkemeyer, Thomas/Kalthoff, Herbert/Rieger-Ladich, Markus (Hrsg.): Bildungspraxis. Körper - Räume - Objekte. Weilerswist: Velbrück, S. 283-308. 
Gruschka, Andreas (2002): Didaktik. Das Kreuz mit der Vermittlung. Wetzlar: Büchse der Pandora.

Hackl, Bernd (2015): Zimmer mit Aussicht. Räumlichkeiten als Medium von Bildungsprozessen. In: Alkemeyer, Thomas/Kalthoff, Herbert/Rieger-Ladich, Markus (Hrsg.): Bildungspraxis. Körper - Räume - Objekte. Weilerswist: Velbrück, S. 131-158.

Hackl, Bernd/Stifter, Alois (2017): In Bewegung. Körperliche Performanz im Unterricht. In: Proske, Matthias/Rabenstein, Kerstin (Hrsg.): Unterricht beobachten - beschreiben - rekonstruieren. Kompendium qualitativer Unterrichtsforschung. Bad Heilbrunn: Klinkhardt (im Erscheinen).

Hausmann, Gottfried (1959): Didaktik als Dramaturgie des Unterrichts. Heidelberg: Quelle \& Meyer.

Helsper, Werner (2008): Schulkulturen - die Schule als symbolische Sinnordnung. In: Zeitschrift für Pädagogik 54, 1, S. 63-80.

Hickethier, Knut (2010): Einführung in die Medienwissenschaft (2. Aufl.). Stuttgart/Weimar: J.B. Metzler.

Hollstein, Oliver (2011): Vom Verstehen zur Verständigung. Die erziehungswissenschaftliche Beobachtung einer pädagogischen Denkform. Frankfurter Beiträge zur Erziehungswissenschaft. Monographien, 13, Frankfurt a.M.: BoD

Kade, Jochen (1997): Vermittelbar/nicht-vermittelbar: Vermitteln: Aneignen. Im Prozeß der Systembildung des Pädagogischen. In: Lenzen, Dieter/Luhmann, Niklas (Hrsg.): Bildung und Weiterbildung im Erziehungssystem. Frankfurt a.M.: Suhrkamp, S. 30-70.

Krämer, Sybille (2004): Was haben ,Performativität‘ und ,Medialität‘ miteinander zu tun? Plädoyer für eine in der ,Aisthetisierung ' gründende Konzeption des Performativen. In: Krämer, Sybille (Hrsg.): Performativität und Medialität. München: Fink, S. 13-32.

Krämer, Sybille (2008): Medium, Bote, Übertragung. Kleine Metaphysik der Medialität. Frankfurt a.M.: Suhrkamp.

Macgilchrist, Felicitas (2017): Zur Medialität des Schulbuchs. In: Proske, Matthias/Rabenstein, Kerstin (Hrsg.): Unterricht beobachten - beschreiben - rekonstruieren. Kompendium qualitativer Unterrichtsforschung. Bad Heilbrunn: Klinkhardt (im Erscheinen).

Martens, Matthias/Asbrand, Barbara/Spieß, Christian (2015): Lernen mit Dingen - Prozesse zirkulierender Referenz im Unterricht. In: Zeitschrift für interpretative Schul- und Unterrichtsforschung 4, S. 48-65.

Mormann, Thomas (1997): Ist der Begriff der Repräsentation obsolet? In: Zeitschrift für philosophische Forschung 51, 3, S. 349-366.

Peters, Maria (2005): Performative Handlungen und biografische Spuren in Kunst und Pädagogik. In: Kunstpädagogische Positionen 11. Hamburg: University Press 2005, [http://hup.sub. uni-hamburg.de/volltexte/2008/36/pdf/HamburgUP_KPP11_Peters.pdf], letzter Zugriff am 16.9.2015.

Prange, Klaus (1989): Pädagogische Erfahrung. Vorträge und Aufsätze zur Anthropologie des Lernens. Weinheim: Deutscher Studien-Verlag.

Prange, Klaus (2005): Die Zeigestruktur der Erziehung. Grundriss der operativen Pädagogik. Paderborn: Schöningh.

Prange, Klaus/Strobel-Eisele, Gabriele (2006): Die Formen des pädagogischen Handelns. Eine Einführung. Stuttgart: Kohlhammer.

Putzier, Eva-Maria (2011): Das Chemieexperiment: Inszenierung im naturwissenschaftlichen Unterricht. In: Schmitt, Reinhold (Hrsg.): Unterricht ist Interaktion. Analysen zur De-factoDidaktik. Mannheim: Institut für deutsche Sprache, S. 69-107.

Putzier, Eva-Maria (2012): Der ,Demonstrationsraum“ als Form der Wahrnehmungsstrukturierung. In: Hausendorf, Heiko/Mondada, Lorenza/Schmitt, Reinhold (Hrsg.): Raum als interaktive Ressource. Tübingen: Narr, S. 275-315. 
Rabenstein, Kerstin (2017): Ding-Praktiken: Zur sozio-materiellen Dimension von Unterricht. In: Proske, Matthias/Dies. (Hrsg.): Unterricht beobachten - beschreiben - rekonstruieren. Kompendium qualitativer Unterrichtsforschung. Bad Heilbrunn: Klinkhardt (im Erscheinen).

Rabenstein, Kerstin \& Wienike, Johanna (2012): Der Blick auf die Dinge des Lernens. Überlegungen zur Beobachtung der materiellen Dimension pädagogischer Praktiken. In: Reh, Sabine/de Boer, Heike (Hrsg.): Beobachtung in der Schule - beobachten lernen. Wiesbaden: Springer VS, S. 189-202.

Rheinberger, Hans Jörg/Hagner, Michael/Wahrig-Schmidt, Bettina (Hrsg.) (1997): Räume des Wissens. Repräsentation, Codierung, Spur. Berlin: Akademie-Verlag.

Rißler, Georg (2015): (Un-)Ordnung und Umordnung - Theoretische und empirische Suchbewegungen zum Verhältnis von Differenz(en), Materialität(en), Raum. In: Budde, Jürgen/ Blasse, Nina/Bossen, Andrea/Rißler, Georg (Hrsg.): Heterogenitätsforschung. Empirische und theoretische Perspektiven. Weinheim und Basel: Beltz Juventa, S. 211-238.

Röhl, Tobias (2013): Dinge des Wissens. Schulunterricht als sozio-materielle Praxis. Stuttgart: Lucius \& Lucius.

Tulodziecki, Gerhard/Herzig, Bardo/Blömeke, Sigrid (2017): Gestaltung von Unterricht. Eine Einführung in die Didaktik (3. überarbeitete und erweiterte Auflage). Bad Heilbrunn: Klinkhardt.

Wirth, Uwe (2002a): Der Performanzbegriff im Spannungsfeld von Illokution, Iteration und Indexikalität. In: Ders. (Hrsg.): Performanz zwischen Sprachphilosophie und Kulturwissenschaften. Frankfurt a.M.: Suhrkamp, S. 9-60.

Wirth, Uwe (Hrsg.) (2002b): Performanz zwischen Sprachphilosophie und Kulturwissenschaften. Frankfurt a.M.: Suhrkamp.

Wulf, Christoph/Zirfas, Jörg (Hrsg.) (2007): Pädagogik des Performativen. Theorien, Methoden, Perspektiven. Weinheim: Beltz.

Wulf, Christoph (2007): Ästhetische Erziehung: Aisthesis - Mimesis - Performativität. Eine Fallstudie. In: Wulf, Christoph/Zirfas, Jörg (Hrsg.): Pädagogik des Performativen. Theorien, Methoden, Perspektiven. Weinheim: Beltz, S. 42-48. 


\title{
Thementeil
}

Johanna Ahlrichs, Felicitas Macgilchrist

\section{Medialität im Geschichtsunterricht: Die Rolle des Schulbuchs beim Vollzug von ,Geschichte“}

\begin{abstract}
Zusammenfassung
Beruhend auf ethnographischer Forschung beschreibt dieser Beitrag zwei allägliche Medienpraktiken, die das, was im schulischen Kontext als ,Geschichte“ bezeichnet wird, performativ hervorbringen. (1) Vergegenständlichen: Beim Lesen im Geschichtsunterricht wird eine körperliche Distanz zum Buch eingenommen; dabei werden die abgedruckten Inhalte als „etwas Handfestes“ mit vermeintlichem Wahrheitscharakter vollzogen. (2) Segmentieren: Durch das Aufteilen und Bereiche-Markieren bei der Schulbuchnutzung werden ,Geschichte“ als segmentierbar, Ereignisse und Prozesse als dekontextualisiert und in sich geschlossen hervorgebracht. Die Medienspezifizität des gedruckten Schulbuchs hat - so eine These dieses Beitrags - eine konstitutive politische Wirkkraft im Geschichtsunterricht, welche die Modalitäten des Denkens, Wahrnehmens und Kommunizierens von bzw. über Autorität und sozialer Ordnung prägen kann.

Schlagwörter: Bildungsmedien, Diskursforschung, Ethnographie, Geschichtsunterricht, Materialität, Medialität, Schulbuch, sozio-materielle Analyse
\end{abstract}

\section{Mediality in History Education: The Role of the Textbook in “Doing History"}

Drawing on ethnographic research, this paper describes two media practices which enact what is called 'history' in a school context. (1) Thingification: When students read in class they adopt a bodily distance to the textbook; the textbook becomes 'something physical' which exudes truthfulness. (2) Segmentation: By marking and segmenting space on the pages, the students' bodies enact history as something which can be segmented, events and processes as decontextualized and autonomous. Overall, the paper suggests that the specific mediality of the printed history textbook has a political 'effectfulness' in history education. This not only presents specific topics and issues as particularly relevant, but also shapes broader modalities of perceiving, thinking and communicating about authority and social orderings.

Keywords: educational media, discourse studies, ethnography, history education, materiality, mediality, textbooks, socio-material analysis

\section{Einleitung}

Was bedeutet es, wenn Schülerinnen und Schüler im Geschichtsunterricht mit Schulbüchern arbeiten? Was für eine Art von ,Geschichte ' wird in Praktiken des Lesens, Blätterns oder Abschreibens hervorgebracht und welche potentielle, politische Wirkkraft entfaltet sich dabei? Diesen Fragen widmet sich der vorliegende Beitrag. Er basiert auf ethnographischer Forschung zu sozio-materiellen Schulbuchpraktiken im Geschichtsunterricht. Damit verortet er sich als Teil der aktuellen, wie Meyer-Drawe es ausdrückt, „Rehabilitierung der Dingwelt, die gegen deren Verwandlung ins bloß Gedachte Einspruch erhebt“" (2012: 24). Diese Rehabilitierung, wie sie in den letzten Jahren zu beob- 
achten ist (vgl. z.B. Gebhard u. a. 2015), ${ }^{1}$ irritiert und provoziert zwar die Unterrichtsforschung in produktiver Weise, aber sie vernachlässigt - so unsere Beobachtung - die politische Dimension, die in anderer Forschung zum Geschichtsunterricht eine wichtige Rolle spielt.

So ist beispielsweise in der Schulbuchforschung eine zentrale Frage, wie über staatlich legitimierte Curricula und Schulbücher bestimmte Geschichtsbilder vermittelt und stabilisiert werden. Dabei geht es vor allem um die Inhalte von Schulbüchern. In diesem Beitrag behalten wir diese politische Dimension bei, lassen aber soweit möglich die Inhalte aus dem Blick ${ }^{2}$ und fokussieren stattdessen auf das Schulbuch als materiellen Gegenstand sowie die „small events“ (Callon 2005: 18): kleinste, anscheinend banale alltägliche Medienpraktiken im Unterricht, bzw. was das Schulbuch macht und was mit ihm gemacht wird. In diesen Praktiken entfaltet sich - so unsere Annahme - eine (politische) „Wirkkraft“, welche über den Geschichtsunterricht hinausgeht und „die Modalitäten unseres Denkens, Wahrnehmens, Erfahrens, Erinnerns und Kommunizierens prägt“" (Krämer 1998: 14).

Nachdem wir in Abschnitt 1 einen Überblick über ausgewählte relevante Forschungsperspektiven gegeben haben, beschreiben wir in Abschnitt 2 die methodische Herangehensweise und klären zentrale Begrifflichkeiten. Abschnitt 3 präsentiert die Analyse anhand zweier Medienpraktiken, die ,Geschichte' nicht nur vermitteln, sondern performativ hervorbringen. Diese These wird in Abschnitt 4 zusammengefasst.

\section{Forschungsstand}

Da wir Unterricht aus einer ethnographischen Perspektive als kulturelle Praxis betrachten, fokussieren wir zum einen auf die Konstitution von Geschichte, zum anderen auf die gesellschaftliche und politische Wirkkraft von Unterrichtsmedien. D. h. neben den empirischen, pragmatischen und normativen Fragen zum historischen Lernen (vgl. Rüsen 2008: 73) greifen wir hier zwei weitere Perspektiven auf, die vor allem die unintendierten, unbemerkten und beiläufigen Prozesse des alltäglichen Unterrichtsgeschehens untersuchen.

\subsection{Politische Dimensionen des Geschichtsunterrichts}

Jede Bezugnahme auf Vergangenes ist zwangsläufig mit Selektionen und deutender Reorganisation verbunden. Vergangenheitsbezügen wird daher auch zugesprochen, dem

1 Neben dem ZISU Thementeil in 2015 gibt es z. B. Sonderhefte zu diesem Thema in der Zeitschrift für Pädagogik (2012), Zeitschrift für Erziehungswissenschaft (2013), Educational Philosophy and Theory (2011), Pedagogy, Culture \& Society (2012) und International Journal of Child, Youth and Family Studies (2014).

2 Die Inhalte können nie gänzlich außer Acht gelassen werden; Inhalt und Form sind bekanntlich miteinander verwoben. Zum analytischen Zweck blenden wir aber - aufgrund des begrenzten Platzes, der für diesen Beitrag vorgesehen ist - die Inhalte der Schulbücher weitgehend aus und hoffen, dadurch die Nutzungspraktiken, die nicht unmittelbar mit bestimmten historischen Ereignissen, Prozessen o. ä. zu tun haben, stärker in den Blick nehmen zu können. 
„Zwecke der Stabilisierung oder Destabilisierung kollektiver Identität“ (Assmann 2002: 414) zu dienen oder die Macht zu haben, gut und schlecht, richtig und falsch, wir und sie zu definieren (vgl. Seixas 2000: 21). Insbesondere Geschichtsunterricht, als staatlich organisierter Bezug auf Vergangenes, hat diese potentiell politische Kraft. Die Frage, wie Vergangenes aktualisiert wird, ist aus dieser Sicht also von entscheidender politischer Bedeutung.

Diese politische Dimension des Geschichtsunterrichts ist für verschiedene Ansätze in der geschichtsdidaktischen und kulturwissenschaftlichen Schulbuchforschung zentral, die hier nur exemplarisch angedeutet werden können (vgl. Handro/Schönemann 2011; Fuchs/Bock im Erscheinen). Schulbuchtexten wird dabei zugesprochen, als „Konstruktionen und zugleich auch Konstrukteure sozialer Ordnungen und gesellschaftlichen Wissens" zu fungieren, dabei Selbst- und Fremdbilder herzustellen sowie bestimmte Perspektiven auf und Versionen der Geschichte zu exkludieren oder abzuwerten (Lässig 2010: 203). Für den Geschichtsunterricht wird u. a. untersucht, wie ,nationale Identität“ oder ,die Anderen“ in Unterrichtsmaterialien dargestellt werden. Zudem wird das Exklusionspotential von Geschichtsschulbüchern kritisiert, z. B. durch die Beobachtung, dass primär nur dominante Perspektiven angeboten und weitere Perspektiven (z. B. der Zugewanderten) ausgeschlossen werden (vgl. Alavi 2004; Beauftragte der Bundesregierung 2015; Lücke 2012; Marmer/Sow 2015).

Solche Studien legen den Fokus auf die inhaltlichen Vermittlungsabsichten, die durch Schulbuchtexte und Curricula an den Geschichtsunterricht herangetragen werden. Was dabei jedoch weitgehend außer Acht bleibt, ist die Frage, was mit den Schulbüchern im Unterricht geschieht (Ausnahmen bilden z.B. Ahlrichs u. a. 2015; Gautschi u. a. 2007). Da davon ausgegangen werden kann, dass sich Unterricht nicht als linearer Vermittlungsprozess vollzieht, in dem bestimmte Absichten die gezielte Wirkung erreichen (vgl. Edwards 2009; Meseth/Proske/Radtke 2012), können vor allem die nicht-intendierten Praktiken von Interesse sein, wenn das Erkenntnisinteresse auf der politischen Dimension liegt. Dazu fordert Barricelli, angesichts der ,ethischen Implikationen“ und der politischen „Aussagekraft“" von Geschichtsdarstellungen, Inhaltsfragen zugunsten von Formfragen zurückzustellen (2012: 221f.). Diese Forderungen aufgreifend widmen wir uns dem Geschichtsunterricht als Ort, an dem nicht nur die Inhalte und Strukturen der Medien, sondern auch eine Beobachtung der Formen/Materialitäten und der Schulbuchpraktiken Einsichten bieten können in die beiläufige Hervorbringung sozialer Ordnungen und damit einhergehenden In- und Exklusionen.

\subsection{Unterricht als performative Praxis}

Ansätze, die helfen, diese Schulbuchpraktiken zu fassen, nehmen Unterricht als performativen Vollzug bzw. als eigenlogische Praxis in den Blick (vgl. z. B. Alkemeyer et al. 2015; Reh et al. 2011). Auf epistemologischer Ebene wird dabei das unterrichtliche Geschehen als kontingent angesehen statt als eine Realisation von konkreten Vermittlungsintentionen. Auf methodischer Ebene gerät die situierte Performativität der unterrichtlichen Praktiken in den Blick, d. h. die Frage, wie etwas namens „Unterricht“ im konkreten praktischen Tun hervorgebracht wird. Das Interesse richtet sich dabei auch auf den Zusammenhang körperlicher und sprachlicher Praktiken und auf ihren wirk- 
lichkeitskonstituierenden bzw. performativen Charakter (vgl. Wulf/Zirfas 2014; Evans 2016).

Aber auch Dingen wird zugesprochen, am Vollzug von Unterricht maßgeblich beteiligt zu sein (vgl. z.B. Alkemeyer et al. 2015: 24; Kalthoff/Röhl 2011). Unterricht vollzieht sich, so diese Ansätze, nicht nur auf sprachlicher Ebene, sondern in einem Zusammenspiel körperlicher, sinnlicher, stofflicher und semiotischer Dimensionen. Für das Fach Englisch stellen z. B. Kress u. a. fest, dass ,however powerful the regulatory frameworks that scaffold [das Fach] may be, they do not determine its nature - for this is not pre-established, but realized in practice" (2005: 13). Das Fach Englisch wird also nicht vermittelt, sondern erst in bestimmten Praktiken vollzogen. Auch der Geschichtsunterricht wurde in seiner ,operativen Eigenständigkeit“ (Hollstein et al. 2002: 18) oder in seiner Materialität (vgl. Martens et al. 2015) betrachtet. Geschichtsunterricht gerät so als Ort in den Blick, an dem ,Geschichte' nicht vermittelt oder gelernt, sondern gemacht wird. Was hierbei jedoch bisher unbeachtet geblieben ist, ist die politische Dimension des praktischen Geschehens, $d$. h. wie die performative Hervorbringung des Faches mit der Stabilisierung oder Irritation sozialer Ordnung zusammenhängt.

\subsection{Das Politische der kleinen Praktiken - eine Synthese}

Wie deutlich geworden sein sollte - und bei aller Gefahr der Verkürzung - nehmen die politisch orientierten Zugänge (Abschnitt 1.1) inhaltliche Fragen in den Blick und analysieren Texte und Deutungsangebote statt Praktiken oder Materialitäten. Die Studien hingegen, die sich mit Unterricht als praktischem Vollzug beschäftigen (Abschnitt 1.2), gehen selten auf die politischen Implikationen ein, sondern konzentrieren sich auf die Beschreibung der Performativität der Mikrosituationen.

In diesem Beitrag verbinden wir diese Ansätze, indem wir nach der politischen Dimension der ,kleinen“ Praktiken des Geschichtsunterrichts fragen. Damit verorten wir uns innerhalb des entstehenden Feldes einer Schul- und Unterrichtsforschung, die darüber reflektiert, dass die Reproduktion von Ungleichheiten und die Formierung des Sicht- und Sagbaren nicht ohne Einbezug der materiellen Ebene gedacht werden kann. Ziel dieses Vorgehens ist, das, was Sørensen (2006) „politics of things“ nennt, für den Geschichtsunterricht empirisch zu fassen: die politischen Implikationen des sozio-materiellen Zusammenspiels von Räumen, Körpern, Dingen und Zeichen (vgl. hierzu auch Kontopodis 2009 und Evans 2016).

\section{Methodische Herangehensweise}

Unsere Analyse basiert auf einer zweijährigen ethnographischen Begleitung des Geschichtsunterrichts einer 9. bzw. 10. Gymnasialklasse zwischen 2013 und 2015. Die erhobenen Daten umfassen Audioaufnahmen vom Unterricht, Feldnotizen und Protokolle, Interviews und informelle Gespräche mit Lehrenden, Schülerinnen und Schülern, Fokusgruppendiskussionen sowie die Analyse von im Unterricht verwendeten Medien.

In Anlehnung an Mohns kamera-ethnographische Studien erstellen wir hier wenngleich in schriftlicher Form - „Porträts, Blickschneisen und Miniaturen“, die sich 
an einem „dichten Zeigen“ orientieren (Mohn/Breidenstein 2013: 5). Kurze Szenen, kreativ aus ihren Kontexten genommen und zusammengeschnitten, ermöglichen „eine Fokussierung der Körperlichkeit pädagogischer Interaktion, der Materialität des Unterrichtsgeschehens und der Choreographie von Personen und Dingen im Klassenzimmer" (ebd.). In diesem Sinne richtet sich unser Blick im Folgenden auf das, was sich neben den sprachlichen, sinnbildenden, abstrahierenden Tätigkeiten des Geschichtsunterrichts vollzieht: Was geschieht, wenn Vergangenes in Schulbuchform und in den damit zusammenhängenden Praktiken des Lesens, Blätterns und Aufteilens präsent gemacht wird?

Als ethnographisch Forschende sind wir mit der Datenerhebung, der Analyse und den Ergebnissen immer schon eng verstrickt. Unsere Auswahl an Szenen hat nicht den Anspruch, ,repräsentativ' zu sein oder universelle ,Wahrheiten' darüber aufzudecken, wie Vergangenes in Unterrichtspraktiken präsent gemacht wird. Eine „Kultur des genauen Hinsehens“ (Schiffauer 2008: 15) soll stattdessen Einsichten in Wie-Fragen schaffen, die über die spezifischen Fälle hinausweisen.

Zwei Begriffe müssen geklärt werden: Medien und Praktiken. Ohne auf die vielfältigen Definitionen im komplexen Feld der Medientheorien einzugehen, beschreiben wir hier ,Medien“ - in Anlehnung an die Medienhistorikerin Gitelman - als soziale Kommunikationsformen, die sowohl technologische Formate als auch die mit diesen Technologien assoziierten Praktiken beinhalten (vgl. Gitelman 2006: 7). Für die Beobachtung von Geschichtsunterricht erscheint uns dieser Medienbegriff zielführender als ein Verständnis von Medien im Sinne von z. B. Trägern oder Speichern, oder Definitionen, die alleine durch die inhaltliche Ebene bzw. die medial übermittelten Informationen operieren, z.B. „Darstellungen“ und „Quellen“ (zur kritischen Diskussion, vgl. die Beiträge in Pallaske 2015). Zum einen ist bei Gitelmans Verständnis von Medien deren Historizität wichtig, die u. a. die in sich wandelnden Nutzungspraktiken zeigt. Zum anderen fängt dieser Medienbegriff die vielfältigen Normen, Konventionen und sozialen, ökonomischen, kulturellen, politischen und materiellen Relationen auf, die mit einer Technologie einhergehen.

Mit Schatzki verstehen wir unter Praktiken ein ,temporally unfolding and spatially dispersed nexus of doings and sayings“ (1996: 89). Soziale Ordnungen, Individualisierungen usw. werden durch Praktiken konstituiert (ebd.: 13). D. h., auch ,Vergangenheit“ und ,Geschichte' konstituieren sich erst in Praktiken (vgl. Barad 2012; Verran 2001). $\mathrm{Da}$,Vergangenheit [...] ebenso wie Geschichte [...] nicht unmittelbar erfahrbar ist“ (Pallaske 2015: 8), sondern immer nur über Interpretationen und Konstruktionen, interessiert uns also neben den sprachlichen Konstruktionen, die bisher im Forschungsfokus standen (vgl. z.B. Coffin 2006), insbesondere die Rolle der alltäglichen, performativen und sozio-materiellen Praktiken, also das Zusammenspiel von Sprache, Dingen, Artefakten, Körpern, Räumen, Nutzungsweisen, implizitem Wissen und Routinen.

\section{Geschichte hervorbringen}

Im Folgenden beschreiben und analysieren wir ausgewählte Zusammenstellungen von Unterrichtszenen, in denen wiederkehrende (Medien-)Praktiken im Geschichtsunterricht im Vordergrund stehen: „Vergegenständlichen“ und „Segmentieren“. 


\subsection{Vergegenständlichen}

Eine zentrale unterrichtliche Tätigkeit in Bezug auf das Schulbuch ist das Lesen. Lesen, ob Darstellung, Quelle oder Fiktion, ist zunächst einmal eine körperliche Aktivität. Um lesen zu können, muss man den Körper in bestimmter Weise ausrichten und seinen Blick auf das Geschriebene fokussieren. Als multimodale Tätigkeit (vgl. Jewitt 2006) ist Lesen dabei ein Zusammenwirken unterschiedlicher Sinne, Haltungen und Gesten. Die sechs folgenden Szenen geben einen Einblick, wie sich diese Körperlichkeit im Unterricht zeigt. Alle Namen sind Pseudonyme:

1) Alle wenden sich wieder dem Buch zu. Sahra hat es auf ihren Beinen aufgestellt, sodass sie ihr Kinn ganz eng an den Brustkorb legen muss, um hineinschauen zu können. Dabei hängt sie tief in ihrem Stuhl und der Blick ist gesenkt. Sie sieht auf diese Weise sehr in sich versunken aus; es ist eine verschlossene Haltung. Aisha hat ihr Buch auf dem Tisch liegen und liest tief darüber gebeugt, Semas Buch liegt auf der Tischkante. Alle vertiefen ihren Blick in das Buch.

2) Lukas hebt das vor ihm liegende Buch an der Oberseite an, sodass es senkrecht auf dem Tisch steht und geht mit seinem Kopf und seinen Augen ganz nah an das Bild heran. Er hängt so tief im Stuhl, dass er sich gar nicht weiter bücken muss.

3) Wieder senken alle den Kopf und schauen in ihre Bücher. 40 Sekunden vergehen. Dann schaut Aisha auf, reckt die Hände in die Höhe, streckt sich und lehnt sich mit dem Oberkörper nach hinten.

4) Melissa liest mit dem Rücken an die Wand gelehnt, seitwärts am Tisch sitzend und das Buch auf dem Schoß. Mit einem Bleistift verfolgt sie die Zeilen.

5) Beim Klassengespräch suchen die Schülerinnen und Schüler teilweise nach der Antwort auf die Lehrerfrage im Buch bzw. vergewissern sich noch einmal, bevor sie sich melden. Bastian hält seinen Finger auf einer bestimmten Textstelle, während er sich meldet, um die Stelle nicht zu verlieren, und liest sie dann vor, als er drangenommen wird.

6) Nach einem Vortrag nimmt Ania ihren Arm hoch, lässt ihn dann wieder sinken und liest noch einmal etwas im Buch nach. Dabei fährt sie mit ihrem Finger über die Zeilen. Dann schaut sie hoch und meldet sich wieder. Die Lehrerin nimmt sie dran. Ania sagt zu den Vortragenden: „Ihr habt auf dem Plakat geschrieben, dass die meisten Frauen Sekretärinnen waren. Das stimmt ja nicht wirklich. Also da steht zwar hier, dass ein paar davon waren, aber ihr habt das so hervorgebracht, als ob alle Frauen, die einen Beruf ausüben wollten, Sekretärinnen wurden. “ Die Lehrerin fragt, was die Frauen noch wurden. Ania antwortet: ,, Also hier stand... “, sie greift das Buch, das auf dem Tisch vor ihr liegt, klappt es hoch, orientiert sich kurz und liest vor.

Die Schülerinnen und Schüler wenden sich hier mit ihrem Oberkörper und Kopf dem Geschriebenen zu und nähern sich diesem, sodass ihre Augen die Schrift gut entziffern können. Die Haltungen sind dabei durchaus unterschiedlich und individuell, aber alle vereint in einem ,Gebeugtsein', einem ,Sich-Vertiefen“. Deutlich wird das Besondere und Geschlossene dieser Haltung vor allem, wenn Aisha in der dritten Szene sich wie- 
der aus ihr löst und vom Gebeugten in eine offene, gestreckte Haltung übergeht. Sahra, Lukas und Aisha wirken beim Lesen mit dem Buch als kleine, in sich geschlossene Einheiten ohne Bezug oder Öffnung nach außen. In der individualisierten Haltung dieser ersten Szenen hat der Sehsinn Priorität - es wird nicht explizit gehorcht, gefühlt oder gerochen, sondern geschaut. Dabei gehen die Schülerinnen und Schüler nicht nur in eine analytische, sondern auch in eine körperliche Distanz zum Schulbuch, um es betrachten zu können. Der Text wird dabei zu einem Gegenüber.

Die Distanz wird aber auch in den Szenen (4) bis (6) durch das Berühren des Schulbuchs verringert. Die Schülerinnen und Schüler ,begreifen“ hier den Text im wörtlichen Sinne, indem sie mit Stiften und Fingern die Lesebewegung ihres Blickes mitzeichnen. Sie stellen einen körperlichen Kontakt zu dem Text her, doch bleibt das Geschriebene auch in seiner Greifbarkeit äußerlich und in sich geschlossen. Für Bastian und Ania ist der Schulbuchtext in dieser Situation präsent als etwas, auf das sie körperlich sowie sprachlich Bezug nehmen. Die Materialität des Textes bietet eine physisch greifbare und betrachtbare Grundlage für das Gesagte.

Im Zusammenspiel von Wort, Geste, Blick, Körperausrichtung und dem Schulbuch als Gegenstand wird auf diese Weise bei Ania das, was „,wirklich stimmt“, als etwas, das im Buch vorzufinden ist, hervorgebracht. Wie die Schülerin Aleksandra in einem Interview sagte: „Und hier hat man eben was Handfestes, wo man immer wieder drin gucken kann: Okay, es ist so gewesen. Und, ja, dass man da eben etwas Handfestes hat" (Interview, 14.07.2015). ${ }^{3}$ Diese Gegen-Ständlichkeit des Geschichtlichen, die in (routinierten) Praktiken des Schulbuchlesens hervorgebracht wird, macht Vergangenes als etwas präsent, das bereits ,da steht“ beziehungsweise als etwas, dem man sich zuwenden kann, das man betrachten und berühren, aber nicht ändern oder beeinflussen kann. Möglicherweise anders wäre dies beispielsweise bei mündlichen Vorträgen, dem Betrachten flüchtiger Bilder bei Filmvorführungen oder einer Internetrecherche. Die Szenen zeigen hier auf, wie die Onto-Epistemologie des Geschichtsunterrichts und die Frage, inwiefern Schülerinnen und Schüler zu Infragestellungen angeregt werden, auch im Zusammenhang mit den verwendeten Medien und den spezifischen Körperpraktiken (Körper beugen, Kopf senken, mit Finger berühren) betrachtet werden muss.

Die Vergegenständlichung von Geschichte, die dem Gelesenen eine Wahrhaftigkeit verleiht, wird zudem unabhängig aller Intentionen der Lehrpersonen oder der Geschichtsdidaktik hervorgebracht. Dies lenkt den Blick weg von der Frage, ob die Lehrperson einen ,besseren“ Unterricht hätte vorbereiten können. Dass Geschichte hier nicht als Deutung von Vergangenem, sondern als etwas, das „so gewesen“ ist, vollzogen wird, scheint mehr mit den „affordances“ (Aufforderungscharakter bzw. Angebot, vgl. Kress 2010) des Schulbuchs und mit den eingespielten Nutzungspraktiken zu tun als mit den Absichten der Lehrperson.

Doch welche Rolle spielen diese „small events“ (Callon), die kleinen, alltäglichen Praktiken der Szenen, in der Aufrechterhaltung oder Veränderung von sozialer

3 Obwohl wir viele solcher Aussagen über Geschichte als „Vergangenheit“, „alles, was passiert ist“, „das, was wirklich mal passiert ist“ usw. von den SuS gehört haben, geben sie gleichzeitig ein differenziertes Bild vom Geschichtsunterricht wider. Letzteres bedeutet „kritisch“ sein, „analysieren“, „nicht direkt alles glauben“, „weitere Quellen nehmen, erforschen“, „zuordnen können“ usw. (alle SuS-Zitate aus Ahlrichs, 2017). 
Ordnung? Zunächst einmal legen sie ein statisches Weltbild nahe. Vergangenheit, die „so gewesen ist“, negiert die Kontingenz jedes historischen, gegenwärtigen und auch zukünftigen Geschehens und engt damit das ein, was für möglich gehalten wird. Zudem beinhaltet ein „so war es“ auch immer ein „so war es nicht“, was alternative Erzählungen abwertet und exkludiert. Aus dieser Sicht ist die Vergegenständlichung der tradierten (dominanten) Geschichtsbilder auch für ihre Wirkkraft auf die soziale Ordnung (Exklusionen und Marginalisierungen) kritisch zu betrachten. Auch allgemeiner: Wenn z. B. Darstellungstexte als handfestes Wissen statt als Deutungen bzw. selektive Versionen behandelt werden, wird ein Vertrauen in Autoritätsfiguren vollzogen. ${ }^{4}$ Dass eine solche Haltung für die Gesellschaft problematisch ist, ist auf inhaltlicher und struktureller Ebene oft beschrieben worden (vgl. z.B. Apple 2000). Wir möchten mit diesem Beitrag die Frage aufwerfen, ob nicht, selbst wenn die Inhalte verändert werden würden, die Handfestigkeit des Schulbuches weiterhin ein Vertrauen in Autorität und somit eine politische Wirkkraft auf die Aufrechterhaltung der gegenwärtigen sozialen Ordnung entfalten würde.

\subsection{Segmentieren}

Die Szenen im folgenden Abschnitt beschreiben Situationen, in denen die Struktur und Gestaltung des Buches bzw. seine Materialität eine entscheidende Rolle in den Nutzungspraktiken gespielt hat.

7) Die Schülerinnen und Schüler werden in Gruppen aufgeteilt und bekommen die Aufgabe, eine Doppelseite im Buch (S. 150f.) zu lesen und ein „Informationsplakat“ zum Thema „Die ,neue“ Frau“ zu erstellen. Nachdem sich die Gruppen zusammengesetzt haben, fangen alle an zu lesen. Plötzlich sagt Sema in die Stille zu ihrer Gruppe: ,Wollen wir nicht aufteilen?" Wenn jeder einen Teil läse, ginge es doch viel schneller. Ihre Gruppe stimmt zu und schon beginnen sie damit, den Text aufzuteilen. Sema zeigt auf Textabsätze und sagt: ,, Also du nimmst das und das und ich nehm diese Seite. "Die Ethnographin fragt, nach welchen Kriterien sie aufteilen. Zusammenhang? „Nee, Textlänge. So, dass alle ungefähr gleich viel haben.“ Die Gruppe erstellt schließlich ein Plakat, auf dem in einer Tabelle Stichpunkte unter den Überschriften ,politisch“, „, beruflich“ und „privat“ stehen.

8) Martin beschreibt in einem Vortrag das Bild auf S. 142, das seiner Gruppe zugeteilt war. Er erzählt, dass während der Inflation von 1923 die Reichsmark praktisch nichts mehr wert war. Die Lehrerin fragt, ob er auch etwas über die Ursachen der Inflation sagen könne. Martin wird etwas verlegen und sagt, das sei auf der Doppelseite 142 und 143 nicht ausführlich erklärt. Die Lehrerin fragt nach S. 141, auf der zwar keine ausführliche, aber doch eine Erklärung gegeben werde. Martin lacht verlegen. Er sagt, er habe das Problem erkannt. Die Gruppe habe das Bild auf S. 142 bekommen und dachte, sie müsse sich also nur auf diese [Doppel]Seite konzentrieren.

4 Andere Schulfächer mögen eine ähnliche Wirkkraft haben. Wir können nur empirische Aussagen über den Geschichtsunterricht treffen; möchten aber keinesfalls behaupten, dass die Vergegenständlichung von ,Wissen“ nur im Geschichtsunterricht passiert. 
9) Während einer Einheit zum Thema Imperialismus sollen die Schülerinnen und Schüler in Partnerarbeit , Informationsplakate “ über ,, das Vorgehen der Kolonialmächte" in verschiedenen Regionen erstellen. Grundlage dafür sind jeweils Abschnitte im Buch. Sema und Martin bekommen S. 55 und 56 (Kolonisierung in Afrika) im Buch zugeteilt. Sie lesen und machen Notizen. Sema hört am Ende von Seite 55 auf zu lesen und plädiert in der anschließenden Diskussion mit Martin dafür, nur das auf dem Plakat zu behandeln, was auf der von ihr gelesenen Seite steht.

In diesen drei Szenen sehen wir exemplarisch die Arbeit des Aufteilens und BereicheMarkierens. In jeder Szene wird pragmatisch und produktorientiert gearbeitet (vgl. Breidenstein 2006): Die Gruppen erarbeiten Poster, Vorträge und Plakate. Die Szenen haben wir ausgewählt, weil die Relevanzsetzungen der Inhalte hier anhand des Layouts und der materiellen Begrenzungen des Schulbuchs sowie der zu erstellenden Produkte vollzogen werden. Semas Gruppe teilt den Text nicht nach inhaltlichen Kriterien auf, sondern so, dass alle ungefähr gleich lange Textbruchstücke zu bearbeiten haben. Martins Gruppe hat sich nicht gefragt, wo die historisch relevanten Informationen zu den Ursachen der Inflation stehen könnten, was vor- oder zurückblättern erfordert hätte, sondern hat sich auf ein sichtbares Segment (Doppelseite) mit dem ihnen zugewiesenen Bild konzentriert. Sema hört mit dem Lesen weder an irgendeiner (freigewählten) Stelle auf noch an einer thematisch/inhaltlichen Grenze, sondern dort, wo der Text eine materielle Begrenzung erfährt.

Was geschieht hier mit Geschichte? Zusammenhänge werden unterbrochen, historische Ereignisse oder Aspekte als in sich geschlossene und voneinander getrennte Segmente präsent gemacht und dabei Ursachen, Folgen oder der weitere Kontext als nicht-relevant vollzogen. In der Konsequenz landen nur bestimmte Aspekte des zu behandelnden Themas auf dem Plakat bzw. im Vortrag.

Segmentierung ist konstitutiv für den schulischen Alltag. Die Aufteilung in Fächer, Klassenstufen, Schulstunden, Themen, Arbeitsblöcke usw. vollzieht von vorneherein Unterbrechungen und Schließungen. Auch Schulbuchinhalte und nicht zuletzt das Verhalten der Lehrpersonen können diesen Prozess entscheidend unterstützen. Wir legen in diesem Beitrag jedoch den Fokus auf die materiell-semiotische Dimension des Geschehens und fragen danach, welche Rolle hier auch die stofflich-sinnlichen Medienpraktiken im Unterricht spielen.

Zunächst ein Blick auf den Aufforderungscharakter des Schulbuchs. Schulbücher weisen gattungs- bzw. medienspezifische Merkmale auf, wie das Doppelseitenprinzip, das graphische Layout, die Strukturierungsmodalitäten (Kapitel - Unterkapitel - Spalte - Überschrift 1 - Überschrift 2 - Absatz - Kasten - Marginalie) usw. (vgl. van Leeuwen 1992; Sauer 2016; Schönemann/Thünemann 2010). Zum einen unterteilt das Buch somit Inhalte auf einzelne, materiell begrenzte Seiten und Doppelseiten und bringt diese als semiotische Einheiten (vgl. Kress 2010: 43) hervor. Zum anderen bilden die darauf sichtbaren Schriften und Graphiken ,nicht einfach ,Texte“, sondern zuerst einmal eine ,Textur': ein Gewebe von räumlichen Relationen“" (Krämer 2005: 52). Die visuellen Unterteilungen des Textes sind dabei als die „Verkörperung seiner gedanklichen Ordnung“ (ebd.: 37) aufzufassen.

Die in den Szenen (7) bis (9) relevant gemachten Seiten des Geschichtsschulbuchs bieten ,Geschichte' somit als strukturierte Ansammlung von in sich geschlossenen Seg- 
menten an. Dieses gestalterische Angebot wird von den Schülerinnen und Schüler angenommen, die sich sowohl körperlich als auch inhaltlich nur bestimmten Teilen des Abgebildeten widmen. Wären die Texte in einem anderen medialen Format zugänglich gewesen, z. B. als digitaler Text mit Hyperlinks, hätte das Lesen keines Weiterblätterns oder Zurückblätterns bedurft, sondern des Klickens, Hoch- und Runterscrollens. Hätte Martins Gruppe eventuell auf einen Link zu den Ursachen der Inflation geklickt und hätte Sema weitergescrollt, wenn sie die Möglichkeit dazu gehabt hätte? Dies ist zumindest denkbar. ${ }^{5}$

Diese Relevanzsetzungen, die hier auch anhand des Layouts und der materiellen Begrenzungen des Schulbuchs sowie der erstellten Produkte erfolgen, haben erstens eine Wirkkraft auf die bearbeiteten Inhalte: Die politische Gleichberechtigung, die berufliche Emanzipation, das Selbstbewusstsein und die Eigenständigkeit von Frauen in der Weimarer Republik werden nebeneinandergestellt, ohne deren Verflechtung miteinander oder mit dem Klassenkampf o. ä. zu thematisieren (Szene 7). Die Inflation von 1923 wird ohne Versailler Vertrag und Reparationsleistung (die auf der Seite davor stehen) verständlich gemacht (Szene 8). Das Vorgehen der Kolonialmächte in Afrika wird mit rassistischen Überlegenheitsgefühlen und Sklavenhandel präsentiert, aber ohne die Privatwirtschaft und den erwarteten Reichtum (die auf der Seite nach der bearbeiteten Seite stehen; Szene 9).

Die Schulbuchpraktiken haben zweitens eine potentiell breitere (politische) Wirkkraft. Die Frage ist, ob der Vollzug von ,Geschichte' als segmentierbar und isolierbar auch Resonanz findet in einem Grundverständnis von globaler sozialer Ordnung. Um nur einen Aspekt aufzugreifen: Verflechtungsgeschichte wird auf der stofflich-sinnlichen Ebene der Arbeit mit Schulbüchern nicht nahegelegt, d. h. die Beziehungen, Transfers und gegenseitige Beeinflussung zwischen Weltregionen oder Prozessen, Personen und Ereignissen finden sich in der Architektur des (linearen, rechteckigen, segmentierten) Buchformats nicht wieder. Die Segmentierbarkeit kann somit auch mit der Unsichtbarkeit von rassismuskritischen oder postkolonialen Themen im Unterricht und im öffentlichen Raum verknüpft sein, z. B. die Verbindungen zwischen der Versklavung in den Kolonien und dem Leben der Frauen in der Weimarer Republik (vgl. Grewe 2016; Marmer/Sow 2015; Völkel 2014).

Die hier beschriebenen Beobachtungen legen die Frage nahe, ob, selbst wenn auf der inhaltlichen Ebene z. B. auf Verflechtungsgeschichte gesetzt würde, dennoch in den alltäglichen Praktiken der Schulbuchnutzung (politisch relevante) Grenzziehungen und Segmentierungen vollzogen würden. Könnte zum Beispiel unabhängig von dem, was vermittelt werden soll, auf der stofflich-sinnlichen Ebene des Unterrichts ein Verständnis einer vermeintlichen Unabhängigkeit des heutigen Deutschlands hervorgebracht werden, das dessen Abhängigkeit von u. a. globalen Rohstoffen und Arbeitskräften exkludiert?

\section{Fazit}

Wir haben in diesem Beitrag ethnographische Forschung beschrieben, die die sozio-materiellen Aspekte des Mediengeschehens im Geschichtsunterricht in den Vordergrund

5 Welche potentiellen Auswirkungen die digitalen Medien auf das historische Lernen haben, ist eine spannende Frage, die den Rahmen dieses Beitrags sprengt. 
der Aufmerksamkeit stellt. Das Geschichtsschulbuch und die Schulbuchnutzung standen im Mittelpunkt der Beobachtungen. Die leitende These des Beitrags ist, dass die Schulbuchpraktiken ,Geschichte ' in spezifischer Weise hervorbringen. Diese Praktiken haben eine politische Wirkkraft: Zum einen in Bezug auf die Relevanzsetzungen für die Themen und Inhalte des Unterrichts, d. h. die Hervorbringung von bestimmten Geschichten mit den dabei vollzogenen In- und Exklusionen, und zum anderen in einem breiteren Sinne, in Bezug auf die - um es mit Krämer (1998) zu sagen - Modalitäten des Denkens, Wahrnehmens, Erfahrens, Erinnerns und Kommunizierens.

Anhand von Szenen aus dem Geschichtsunterricht haben wir aufgezeigt, wie Schulbuchpraktiken dem Geschriebenen eine Autorität verleihen und ,Geschichte“ vergegenständlichen und segmentieren. Ohne hier Vollständigkeit zu beanspruchen, wird das Fach Geschichte in diesen Szenen als ein Fach hervorgebracht, in dem man ,Geschichte" als das vollzieht, was ,gewesen ist“" (Aleksandra), und Ereignisse, Gruppen und Prozesse segmentiert sowie isoliert voneinander behandelt. Jede dieser Tätigkeiten des Geschichtsunterrichts ist kritisiert worden, auch innerhalb der Geschichtsdidaktik. Mit den „Blickschneisen und Miniaturen“ zeigen wir auf, dass diese Kritik nicht nur auf der inhaltlichen Ebene, sondern auch eine Ebene tiefer in der „Medienarchäologie“ (vgl. Parikka 2012) des Unterrichts anzusetzen ist. Unser Anliegen ist es, die konstitutive Rolle der Materialität der Unterrichtsmedien, die meistens unsichtbar bleibt, sichtbar zu machen. Die ,small events“, wie das Sich-Beugen über ein Schulbuch, das Umblättern oder das Nicht-Umblättern, konstituieren Geschichte in besonderer Weise und entfalten eine subtile Wirkkraft, die die Modalitäten des Sicht- und Sagbaren prägt.

\section{Autorenangaben}

Johanna Ahlrichs, M.A.

Georg-Eckert-Institut - Leibniz Institut für inter-

Prof. Dr. habil. Felicitas Macgilchrist

nationale Schulbuchforschung

Celler Str. 3

38114 Braunschweig

053159099223

ahlrichs@leibniz-gei.de

Georg-August-Universität Göttingen und

Georg-Eckert-Institut - Leibniz Institut für

internationale Schulbuchforschung

Celler Str. 3

38114 Braunschweig

053159099283

macgilchrist@leibniz-gei.de

\section{Literatur}

Ahlrichs, Johanna (2017): Die Darstellung von Ungleichheit. Geschichtsunterricht und die Relevanz des Beiläufigen. Diss. Mainz: Universität Mainz/Institut für Soziologie.

Ahlrichs, Johanna/Baier, Katharina/Christophe, Barbara/Macgilchrist, Felicitas/Mielke, Patrick/ Richtera, Roman (2015): Memory practices in the classroom. On reproducing, destabilizing and interrupting majority memories. In: Journal of Educational Media, Memory, and Society 7, 2, S. 89-109.

Alavi, Bettina (2004): Geschichtsschulbücher als Erinnerungsorte. Ein Gedächtnis für die Einwanderungsgesellschaft. In: Motte, J./Ohliger, R. (Hrsg.): Geschichte und Gedächtnis 
in der Einwanderungsgesellschaft. Migration zwischen historischer Rekonstruktion und Erinnerungspolitik. Essen: Klartext, S. 199-212.

Alkemeyer, Thomas/Kalthoff, Herbert/Rieger-Ladich, Markus (Hrsg.) (2015): Bildungspraxis. Körper - Räume - Objekte. Weilerswist: Velbrück Wissenschaft.

Apple, Michael W. (2000): Official Knowledge. New York: Routledge.

Assmann, Jan (2002): Nachwort. In: Esposito, E. (Hrsg.): Soziales Vergessen. Formen und Medien des Gedächtnisses der Gesellschaft. Frankfurt am Main: Suhrkamp, S. 400-414.

Barad, Karen (2012): Agentieller Realismus. Berlin: Suhrkamp.

Barricelli, Michele (2012): Darstellungskonzepte von Geschichte im Unterricht. In: Barricelli, M./Lücke, M. (Hrsg.): Handbuch Praxis des Geschichtsunterrichts, Band I. Schwalbach/Ts: Wochenschau, S. 202-223.

Bock, Annekatrin/Niehaus, Inga/Tribukait, Maren (2015): Abschlussbericht. Verwendung elektronischer Bildungsmedieninhalte in Braunschweiger Notebook-Klassen. Durchgeführt vom Georg-Eckert-Institut - Leibniz-Institut für internationale Schulbuchforschung. Eckert. Working Papers 2015/5. [http://www.edumeres.net/urn/urn:nbn:de:0220-2015-00109], Zugriff am 16.12.2016.

Breidenstein, Georg (2006): Teilnahme am Unterricht. Ethnographische Studien zum Schülerjob. Wiesbaden: VS Verlag für Sozialwissenschaften.

BeauftragtederBundesregierung fürMigration,FlüchtlingeundIntegration(2015): Schulbuchstudie Migration und Integration. Berlin. [http://repository.gei.de/handle/11428/65], Zugriff am 19.01.2017.

Callon, Michel (2005): Why Virtualism Paves the Way to Political Impotence. A Reply to Daniel Miller's Critique of The Laws of the Markets. In: Economic Sociology 6, 2, S. 3-20.

Coffin, Caroline (2006): Historical Discourse. The Language of Time, Cause and Evaluation. London: Continuum International.

Edwards, Richard (2009): Translating the Prescribed into the Enacted Curriculum in College and School. In: Educational Philosophy and Theory, 43, 1, S. 38-54.

Evans, Katherine (2016): Beyond a logic of quality: Opening space for material-discursive practices of 'readiness' in early years education. In: Contemporary Issues in Early Childhood 17, 1, S. 65-77.

Fuchs, Eckhardt/Bock, Annekatrin (im Erscheinen): Palgrave Handbook of Textbook Studies. London: Palgrave.

Gautschi, Peter/Moser, Daniel/Kurt, Reusser/Wiher, Pit (Hrsg.) (2007): Geschichtsunterricht heute. Eine empirische Analyse ausgewählter Aspekte. Bern: h.e.p.

Gebhard, Ulrich/Hummrich, Merle/Rabenstein, Kerstin/Reh, Sabine (2015): Räume, Dinge und schulisches Wissen. Eine Einführung In: Zeitschrift für interpretative Schul- und Unterrichtsforschung 4, 4, S. 3-14.

Gitelman, Lisa (2006): Always Already New. Media, History, and the Data of Culture. Cambridge, Mass. u. a.: MIT Press.

Grewe, Bernd-Stefan (2016): Geschichtsdidaktik postkolonial. In: Zeitschrift für Geschichtsdidaktik 15, 1, S. 5-30.

Handro, Saskia/Schönemann, Bernd (2011): Geschichtsdidaktische Schulbuchforschung. Münster: LIT.

Hollstein, Oliver/Proske, Matthias/Meseth, Wolfgang/Radtke, Frank-Olaf (2002): Nationalsozialismus im Geschichtsunterricht. Beobachtungen unterrichtlicher Kommunikation: Bericht zu einer Pilotstudie. Frankfurt am Main: Fachbereich Erziehungswissenschaften der Johann Wolfgang Goethe-Universität.

Jewitt, Carey (2006): Technology, Literacy, Learning. A Multimodal Approach. London: Routledge. 
Kalthoff, Herbert/Röhl, Tobias (2011): Interobjectivity and Interactivity: Material Objects and Discourse in Class. In: Human Studies 34, 4, S. 451-469.

Kontopodis, Michalis (2009): Documents' memories. Enacting pasts and futures at the School for Individual Learning-in-Practice. In: Memory Studies 2, 1, S. 11-26.

Krämer, Sybille (1998): Was haben Medien, der Computer und die Realität miteinander zu tun? In: Krämer, S. (Hrsg.): Medien - Computer - Realität. Wirklichkeitsvorstellungen und Neue Medien. Frankfurt am Main: Suhrkamp, S. 9-26.

Krämer, Sybille (2005): ,Operationsraum Schrift‘. Über einen Perspektivenwechsel in der Betrachtung der Schrift. In: Grube, G./Kogge, W./Krämer, S. (Hrsg.): Schrift. Kulturtechnik zwischen Auge, Hand und Maschine. München: Wilhelm Fink, S. 23-60.

Kress, Gunther (2010): Multimodality. A social semiotic approach to contemporary communication. London und New York: Routledge.

Kress, Gunther/Jewitt, Carey/Bourne, Jill/Franks, Anton/Hardcastle, John/Jones, Ken/Reid, Euan (2005): English in Urban Classrooms. A multimodal perspective on teaching and learning. London: Routledge.

Lässig, Simone (2010): Wer definiert relevantes Wissen? Schulbücher und ihr gesellschaftlicher Kontext. In: Fuchs, E./Kahlert, J./Sandfuchs, U. (Hrsg.): Schulbuch konkret. Kontexte Produktion - Unterricht. Bad Heilbrunn: Klinkhardt.

Lücke, Martin (2012): Multiperspektivität, Kontroversität, Pluralität. In: Barricelli, M./Lücke, M. (Hrsg.): Handbuch Praxis des Geschichtsunterrichts, Band I. Schwalbach/Ts: Wochenschau, S. 281-288.

Marmer, Elina/Sow, Papa (2015): Wie Rassismus aus Schulbüchern spricht. Weinheim: Beltz Juventa.

Martens, Mathias/Asbrand, Barbara/Spieß, Christian (2015): Lernen mit Dingen. Prozesse zirkulierender Referenz im Unterricht. In: Zeitschrift für interpretative Schul- und Unterrichtsforschung 4, 4, S. 48-65.

Meseth, Wolfgang/Proske, Matthias/Radtke, Frank-Olaf (2012): Kontrolliertes Laissez-faire. Auf dem Weg zu einer kontingenzgewärtigen Unterrichtstheorie. In: Zeitschrift für Pädagogik 58,2 , S. 223-241.

Meyer-Drawe, Käte (2012): Empfänglichsein für die Welt. Ein Beitrag zur Bildungstheorie. In: Dörpinghaus, A./Nießeler, A. (Hrsg.): Dinge in der Welt der Bildung. Bildung in der Welt der Dinge. Würzburg: Königshaus \& Neumann, S. 13-28.

Mohn, Bina Elisabeth/Breidenstein, Georg (2013): Arbeitswelten in der Grundschule. Praktiken der Individualisierung von Unterricht. Göttingen: Institut für Visuelle Ethnographie.

Pallaske, Christoph (Ed.) (2015): Medien machen Geschichte. Neue Anforderungen an den geschichtsdidaktischen Medienbegriff im digitalen Wandel. Geschichtsdidaktische Studien, Band 2. Berlin: Logos.

Parikka, Jussi (2012): What is Media Archaeology? Cambridge: Polity Press.

Reh, Sabine/Rabenstein, Kerstin/Idel, Till-Sebastian (2011): Unterricht als pädagogische Ordnung. Eine praxistheoretische Perspektive. In: Meseth, W./Proske, M./Radtke, F.-O. (Hrsg.): Unterrichtstheorien in Forschung und Lehre. Bad Heilbrunn: Klinkhardt, S. 209-222.

Rüsen, Jörn (2008): Historisches Lernen. Grundlagen und Paradigmen. 2. Aufl. Schwalbach/Ts: Wochenschau.

Sauer, Michael (2016): Schulgeschichtsbücher: Herstellung, Konzepte, Unterrichtseinsatz. In: Geschichte in Wissenschaft und Unterricht 67, 9/10, S. 588-603.

Schatzki, Theodore R. (1996): Social Practices. Cambridge: Cambridge University Press.

Schiffauer, Werner (2008): Parallelgesellschaften. Bielefeld: transcript.

Schönemann, Bernd/Thünemann, Holger (2010): Schulbucharbeit. Das Geschichtslehrbuch in der Unterrichtspraxis. Schwalbach/Ts: Wochenschau. 
Seixas, Peter (2000): Schweigen! Die Kinder! or does postmodern history have a place in the schools? In: Stearns, P. /Seixas, P./Wineburg, S. (Hrsg.): Knowing, Teaching, and Learning History. National and international perspectives. New York: New York University Press, S. 19-37.

Sørensen, Estrid (2006): Politics of Things: Interplay of Design and Practice in a Design Workshop with Children. In: Costall, A./Dreier, O. (Hrsg.): Doing Things with Things. The Design and Use of Everyday Objects. Adlershot: Ashgate, S. 147-163.

Van Leeuwen, Theo (1992): The schoolbook as a multimodal text. In: Internationale Schulbuchforschung 14, 1, S. 35-58.

Verran, Helen (2001): Science and an African Logic. Chicago: University of Chicago Press.

Völkel, Bärbel (2014): Nationalism - Ethnicity - Racism. Thinking History in a World of Nations. In: Review of History and Political Science 2, 1, S. 29-50.

Wulf, Christoph/Zirfas, Jörg (2014): Performativität. In: Wulf, C./Zirfas, J. (Hrsg.): Handbuch Pädagogische Anthropologie. Wiesbaden: Springer VS, S. 515-526. 
Maria Hallitzky, Beate Beyer, Christopher Hempel, Johanna Leicht, Ellen Schroeter

\section{,Das Märchen von dem Machandelbaum‘ oder wie Unterrichtsstoff durch Medien inszeniert wird}

\section{Zusammenfassung}

Ausgehend von einer theoretischen Auseinandersetzung mit dem Medienbegriff stellt der Beitrag in performativer Analyseeinstellung die empirische Frage nach der Funktion und Bedeutung von Medien in der Unterrichtsinteraktion. Dazu wird eine exemplarisch ausgewählte Unterrichtsszene aus dem Literaturunterricht, die sich durch den gleichzeitigen Einsatz mehrerer Medien auszeichnet, sequenzanalytisch rekonstruiert und didaktisch reflektiert. Es zeigt sich, dass die Medien in der Unterrichtssituation als Autorität inszeniert werden, mit der sich die Schülerinnen zur Lösung ihrer Verstehensprobleme auseinandersetzen müssen. Die Lehrerin delegiert inhaltliche Aspekte an die Medien und beschränkt sich auf die Eröffnung formaler Zugänge - was Selbstständigkeit ermöglicht, aber Überforderung riskiert.

Schlagwörter: Medien, Literaturunterricht, Unterrichtsinteraktion, Lernunterstützung, Dokumentarische Methode

\section{“The Fairy Tale Of The Juniper Tree” or How The Subject Is Framed Through Use Of Media}

Based on a theoretical debate about the concept of media, this article empirically examines the function and relevance of media in classroom interactions. To do so, we analyze an exemplary selected classroom situation about Goethe's Faust, which is characterized by the simultaneous use of multiple media. We show that the media are staged as an authority with which the students have to deal with to solve their problems of understanding. The teacher is responsible only for the formal access to information and not for substantive support. This allows independent learning, while also risking excessive demands on the students.

Keywords: media, literature classes, classroom interaction, learning support, documentary method

\section{Theoretische Anknüpfungspunkte und Fragestellung}

„Ein Medium ist ein Mittel zur Information.“ So lapidar leitet Stefan Münker (2009: 39) seinen philosophischen Beitrag zur medientheoretischen Debatte um die Frage „Was ist ein Medium" ein und verweist damit auf die mit der Funktion des Daten- und Informationstransfers verbundene Deutung von Medien als Mittler (vgl. Krämer 2008: 103; ähnlich Tulodziecki u. a. 2010). Dies steht einem umgangssprachlichen Verständnis von Medien als ,Realobjekten' entgegen und verweist auf einen Beziehungs- oder Funktionsbegriff (vgl. Kerlen 2003: 9). Dieses Verständnis ist aus der Einsicht gespeist, dass unser Verhältnis zu uns selbst, zu den anderen und zur Welt durch eine Mittelbarkeit charakterisiert ist, die wesentlich auf Übertragungsvorgängen beruht (vgl. Münker 2009: 39). Sybille Krämer (2008: 108ff.) verwendet den Begriff von Medien als „Boten“" und betont damit, wie Münker, die Position des Mediums als Mittler im Sinne der ,Mitte' eines Transferprozesses. Mit dem Bild des Boten ist in der unterrichtlichen Beschreibung von Medien insbesondere die Funktion des „Wahrnehmbarmachen(s)“ (Krämer 2008: 104) relevant. 
Unter Rekurs auf die medienkritische Debatte des „Medial Turn“ wird der Begriff des Mediums auch in einer sinnkonstitutiven Funktion als ,strukturgebende und ordnungsstiftende Kraft" (Krämer 2004a: 18) und damit als performativ wirksam wahrgenommen, indem Medien das, was sie übertragen, zugleich auch hervorbringen oder vollziehen (vgl. Krämer 2004b: 23). Damit sind repräsentative Qualitäten der Wahrnehmbarmachung und zugleich konstruktive Qualitäten der Wirklichkeitskonstitution durch Medien (vgl. ebd.) bestimmt. Letzteres nimmt in einer performativen Deutung nicht einseitig auf einen Sender oder eine als objektiv verstandene Botschaft Bezug, sondern setzt Ereignis und Wahrnehmung, Machen und Rezipieren in ein Wechselverhältnis (vgl. ebd.: 21).

Insbesondere für den Unterricht gilt, dass Medien in Form ,technischer Artefakte „nicht einfach da“ (Münker 2009: 47) sind, sondern einerseits gezielt, andererseits auch spontan in die Lernsituationen eingebunden werden, sodass sich die Bedeutung der jeweilig , aufscheinenden“ Medien erst situativ im Handlungsverlauf herausarbeiten lässt. Selbst oder insbesondere wenn man davon ausgeht, dass Medien ,von uns gemachte, technische Artefakte" (ebd.) sind, stellt sich unter der beschriebenen Annahme einer doppelten Konstitutionsleistung die Frage, wie verschiedene Medien durch ihr je besonderes Arrangement im Unterricht an der Konstitution desselben ebenso wie an der Konstitution des zu verhandelnden Gegenstandes beteiligt sind und ggf. zusammenspielen, also wie Medien von LehrerInnen und SchülerInnen verwendet werden und welche Möglichkeiten der Weltdeutung mit ihrer Verwendung realisiert werden (können).

Damit steckt in einem (artifiziellen Unterrichts-)Medium für die SchülerInnen nicht nur der Gegenstand der Aneignung, sondern potenziell auch der/die LehrerIn in der von ihm/ihr geleisteten Vermittlungsarbeit, nämlich der Gestaltung und Auswahl von Medien und der Organisation der Arbeit mit den gewählten Medien (vgl. Sünkel 1996: 161). Und obgleich das ,Wie' der Nutzung, also die durch die Medien ermöglichte Aneignungsarbeit letztlich allein bei den SchülerInnen liegt, sind es die LehrerInnen, die - gemäß dem didaktischen Dreieck - gewissermaßen als personales ,Medium“ vermittelnd zwischen den Unterrichtsgegenstand und die durch ein (Unterrichts)Medium, also das didaktische Material, auf eine über den Gegenstand hinausweisende ,Sache“ oder ,Welt' treten. Sie können mit der Wahl eines Mediums den Blick auf die Sache eröffnen, fokussieren, aber in einer nicht adäquaten Auswahl des Mediums oder (z. B. einer überdidaktisierenden) Form seiner Bearbeitung (vgl. Gruschka 2005) diesen Blick auch verstellen oder ablenken.

Didaktisch bedeutsam ist für uns zunächst nicht die Frage, welche Unterrichtsmedien wie von der Lehrkraft instrumentell eingesetzt werden, sondern vielmehr, welche Rolle Medien in der Herstellung eines gemeinsamen Lerngegenstandes situativ einnehmen. Wir beschränken uns demnach nicht auf bestimmte Formen von Medien oder Fragen nach deren adäquatem Einsatz, sondern folgen dem performativen Vollzug im Unterrichtsgeschehen und richten unseren Fokus darauf, wie Medien im diskursiven Zusammenspiel mit Akteuren den Unterrichtsgegenstand situativ inszenieren und mitgestalten und welche Funktionen im Interaktionsverlauf damit einhergehen, um daraus prozedurale Regeln des konkreten Umgangs und damit einhergehende Relationen von Akteuren und Informationen beschreiben zu können (vgl. Deterding 2013: 51).

Im Literaturunterricht stellt sich die Frage nach Medien insofern nochmals unter einer erweiterten Perspektive auf den Gegenstand, also den Text. Dieser ist - analog zur Sprache als Inhalt und Medium der Reflexion in der Sprachphilosophie - zugleich Inhalt und Me- 
dium des Unterrichts. Der Gegenstand „Text“ lässt sich damit im Literaturunterricht nicht wie ein Medium neben anderen behandeln. Situativ wird er zwar in mehrfacher Hinsicht zu einem Medium in der Funktion eines „Boten“: Er verweist inhaltlich auf eine vor- und außertextliche Welt und stellt - in der Situation des Unterrichts - didaktisch einen Zusammenhang her zwischen eben diesem Inhalt und den in die Situation eingebundenen Akteuren. Neben den Wirklichkeitsbezug eines Textes kann aber zudem auch eine Bezugnahme auf andere Texte treten (vgl. Berndt/Tonger-Erk 2013: 7), die wiederum je ein spezifisches Bezugsfeld erschließen, sodass eine kaskadenförmige Struktur textueller und medialer Bezugnahmen beschreibbar wird. Diese Kaskadenhaftigkeit erhöht sich in ihrer Komplexität dadurch, dass zu der Relation ,Text - Autor - Rezipient' die didaktische Vermittlung durch die Lehrkraft und damit zur literarischen eine didaktische Medialität hinzutritt bzw. erstere umfasst, also die Wahrnehmung des literarischen Inhalts nochmals neu rahmt.

Die moderierend-performative Funktion der medialen Inszenierung (nachfolgend am Beispiel von Textverweisen aus Goethes „Faust“ auf das Märchen vom Machandelbaum dargestellt) bezieht sich sowohl auf die Konstitution der Situation als auch auf die in dieser Situation hervorgebrachten Perspektiven der Beteiligten auf den Gegenstand bzw. auf die in diesem Gegenstand wahrgenommene vor- und außertextliche Welt. Der Zusammenhang von Textualität und Medialität wird dabei so verstanden, dass - einem weiten Textverständnis entsprechend, das Text als einen bedeutungsgenerativen Verweisungszusammenhang bestimmt - kein bestimmtes Medium (auditiv, visuell, analog, digital, ...) vorausgesetzt ist, um von einem Text zu sprechen (vgl. ebd.: 8f.). Berndt und Tonger-Erk (ebd.) schließen hieraus auf eine gewisse „Medienblindheit“" von Intertextualitätstheorien. Zwar haben nicht nur die Texte selbst, sondern auch unterschiedliche ,Transportmittel' (wie Papier oder das Tablet) performative Bedeutung im Hinblick auf die situative Nutzung und Nutzbarkeit durch die Rezipienten. Mit der Präsentation literarischer Texte in einem digitalen Format beispielsweise erfolgt eine intermediale Transformation (vgl. Hickethier 2013; Wirth 2007), die den Einsatz dieser Texte situativ wiederum (performativ) rahmt. Die ,Transportmittel' selbst prägen aber in der Regel keine eigengültige Sinneinheit des Handelns aus, sodass mit Deterding (2013: 57) gefolgert werden kann, dass ,(p)rimär verstehens- und handlungsleitend ist, was wir tun, nicht womit“". Dabei gilt für den Literaturunterricht der besondere Anspruch, sowohl die Mehrdeutigkeit des literarischen Textes als auch die wiederum mehrdeutigen Perspektiven der SchülerInnen auf diesen Text zu konstituieren. Das impliziert zum einen eine Offenheit für Deutungen durch die SchülerInnen, steht aber zum anderen in Spannung zu schulisch geforderten Vereindeutigungen (vgl. grundsätzlich Pflugmacher 2015).

Vor dem Hintergrund des Zusammenspiels von repräsentativen und performativen Potenzialen von Medien stehen für uns folgende Forschungsfragen im Vordergrund:

1. Wie werden literarische Texte im Unterricht medial inszeniert?

2. Welche Funktionen nehmen die Medien bzw. die Art und Weise ihrer Nutzung in der interaktiven Aushandlung und Konstitution des Unterrichtsgegenstandes ein?

Diese Fragen werden in unserem Beitrag anhand einer Einzelfallanalyse bearbeitet. Dabei geht es insbesondere darum, die eben entfaltete und auf die Mediennutzung fokussierte theoretische Perspektive explorativ anzuwenden, gleichzeitig aber auch darum, 
einen von bisherigen Rekonstruktionen (siehe unten) abweichenden Fall hinsichtlich des modus operandi der unterrichtlichen Aushandlung literarischer Texte darzustellen.

\section{Methodisches Vorgehen}

In unseren empirischen Analysen des Literaturunterrichts rekonstruieren wir die Interaktionspraxis in ihrer sequenziellen Struktur und bezogen auf die übergreifende Frage nach dem Umgang mit der Mehrdeutigkeit des unterrichtlichen Gegenstandes (vgl. Hallitzky u. a. 2016). Den oben dargestellten theoretischen Annahmen entsprechend fokussieren wir die Analyse in diesem Beitrag auf die Praxis des Umgangs mit Medien innerhalb einer Unterrichtssituation sowie auf die Bedingungen dieser Praxis als der ,performativen Struktur“, die sich im Vollzug der Interaktion dokumentiert (vgl. Bohnsack 2007). Gegenstand der Analyse ist eine abgeschlossene Interaktionssequenz, die mit einer gegenseitigen Adressierung und Re-Adressierung von Lehrperson und Lerngruppe initiiert wird und sich auf die Bearbeitung einer die Interaktion rahmende Aufgabenstellung bezieht (vgl. Hallitzky u. a. 2016). Die Rekonstruktion eines so bestimmten Falls, der als ,,sachliche Grundlage der reflexiven Auseinandersetzung mit sozialer Wirklichkeit" (Hummrich 2016: 32) fungiert, ermöglicht ein Verstehen der Eigenlogik unterrichtlicher Situationen - hier insbesondere der Bedeutung der Medien für die gemeinsame Aushandlung des Unterrichtsgegenstandes.

Grundlage der Fallauswahl ist die videographische Aufzeichnung von Unterrichtsstunden zum Lernbereich ,Faust' an Sekundarschulen aus zwei Kameraperspektiven, wobei eine Kamera statisch auf die Lerngruppe gerichtet ist und die andere den Bewegungen der Lehrperson folgt (vgl. Fritzsche/Wagner-Willi 2013; Martens u. a. 2015). Die hier ausgewählte Interaktionssequenz ist für eine explorative Untersuchung der oben genannten Forschungsfragen geeignet, da sie sich durch den gleichzeitigen Einsatz mehrerer Medien auszeichnet. Gleichzeitig zeigt sich auf der Oberflächenebene, die über die Grobtranskription der Unterrichtsstunden erfasst wurde, eine geringe Strukturierung der Situation durch die Interaktionsbeiträge der Lehrerin. Die Sequenz steht damit als Fall kontrastiv zu den Ergebnissen bisheriger Fallrekonstruktionen im Literaturunterricht, in denen sich starke Praktiken der ,Vereindeutigung' des unterrichtlichen Gegenstandes durch die Lehrperson zeigen: Die (Be-)Deutung des literarischen Textes wird als eine eindeutige markiert und SchülerInnengruppen werden in Prozessen des ,Nachsteuerns' zu einem entsprechend, richtigen' Verständnis hin gelenkt (vgl. Hallitzky u. a. 2016; auch Reh/Rabenstein 2013 und Pflugmacher 2015).

Bei der Interpretation der ausgewählten Szenen orientieren wir uns methodisch am Vorschlag von Martens, Petersen und Asbrand (2015), die die dokumentarische Methode als „sequenzielle Interaktionsanalyse“ auf videographisch erhobene Unterrichtsprozesse anwenden und notwendige Anpassungen vornehmen. Die praxeologisch-wissenssoziologisch fundierte dokumentarische Methode erscheint für unser Erkenntnisinteresse deshalb geeignet, da sie sowohl eine Rekonstruktion der Praxis - wie wir sie bisher als Interaktionsanalyse betrieben haben - als auch der ihr zugrundeliegenden Rahmungen erlaubt (vgl. grundlegend Bohnsack 2014). Sowohl die Transkription der Szene als auch die formulierende Interpretation erfolgen demnach in tabellarischer Form, wobei 
verbale und nonverbale Interaktionen, zunächst durch Standbilder dargestellt, unterschieden werden. Bei der formulierenden Interpretation wird der Inhalt der Interaktion, der allgemein verständliche Sinngehalt des Geschehens reformuliert bzw. beschrieben. Anschließend erfolgt die reflektierende Interpretation zur „Rekonstruktion des modus operandi der Interaktion der abgebildeten Akteure miteinander oder mit den Dingen, der Körperhaltungen, Gesten und der Mimik“ (Martens u. a. 2015: 193). Es geht darum, in der Analyse der Art und Weise der Bezugnahme der am Unterricht Beteiligten aufeinander sowie auf die einbezogenen Medien die Orientierungen zu rekonstruieren, die als performative Struktur das habituelle Handeln im Unterricht hervorbringen (vgl. Bohnsack 2007; auch Deterding 2013), wobei bei diesem Analyseschritt die verbale und nonverbale Ebene von Beginn an integriert werden.

Wir gehen dabei strikt sequenziell vor und fokussieren die Anschlüsse an einzelne Interaktionsbeiträge vor dem Hintergrund der Mehrdeutigkeit einzelner Äußerungen und folglich unterschiedlicher Anschlussmöglichkeiten. Dabei steht die hier vor allem in explorativer Absicht vorgenommene Fallanalyse in Spannung zur konstitutiven Bedeutung des Vergleichs in der dokumentarischen Methode. Neben den Gegenhorizonten, die aus bisherigen, allerdings nicht auf die Bedeutung der Medien bezogenen Fallrekonstruktionen vorliegen (vgl. Hallitzky u. a. 2016), suchen wir daher vor allem nach „Gemeinsamkeiten innerhalb dieser einen [...] Sequenz" (Nentwig-Gesemann 2014: 124), nach ,wiederkehrende[n], homologe[n] Interaktions- und Orientierungsmuster[n], die der Herstellung der Situation, so wie sie sich entfaltet, zugrundeliegen“ (ebd.).

\section{Fallrekonstruktion}

Die von uns interpretierte Unterrichtsszene ist Teil einer Gruppenarbeitsphase zur „Gretchentragödie“ in Goethes „Faust I“. Die Lehrerin verteilt zu Beginn der Stunde Aufgaben für die Gruppenarbeiten in Form von Arbeitsblättern, die dem Portal ,Lehrer online' 1 entnommen wurden. Eine Schülerinnengruppe, die im weiteren Verlauf im Fokus steht, erhält folgende Aufgabe:

\section{Kerker und Ende von Faust II}

Lesen Sie die Szene Kerker und achten Sie dabei besonders auf das Lied in den Versen 44124420! Zu diesem Lied schreibt ein Kommentator: „Wie mischt sich hier Erlebtes, Kindliches, Magisches! Kein Satz des Sinns könnte so sehr wie der Wahnsinn diese reine Symbolik der Bilder und Klänge erreichen. Aus dem Bereich des Märchens, den Goethe ganz nebenher kennen gelernt hatte, ergreift er gerade das, was wie nichts anderes hierher passt [...]. Die Sicherheit und Selbständigkeit des Griffs und die Fülle der Weltkenntnis bleibt immer wieder bewundernswert - und damit ist an den eigentlichen Zauber der Szene, ihre künstlerische Fügung und menschliche Größe, noch gar nicht gerührt.“ (Erich Trunz in: Goethe. Faust. Der Tragödie erster und zweiter Teil. Urfaust. Hg. u. Komm. v. Erich Trunz. München1986, S. 580)

Überprüfen Sie diese Aussage und nehmen Sie dazu Stellung (ca. 1/2 Seite)!

Das Märchen „Von dem Machandelbaum“ finden Sie an der Adresse

http://www.hekaya.de/maerchen/von-dem-machandelbaum--grimm_khm_47.html

1 Das Material findet sich online unter: http://www.lehrer-online.de/faust.php (aufgerufen am 16.08.2016). 
Im Vergleich zu den anderen Gruppenaufgaben erscheint die Aufgabe, eine Stellungnahme zu einem literaturwissenschaftlichen Kommentar zu verfassen, sehr herausfordernd. Die Komplexität liegt darin, dass verschiedene Textbezüge hergestellt werden sollen, die kaskadenhaft auf spezifische Bezugsrahmen verweisen, welche zunächst im Verborgenen bleiben. Als Texte dienen die Gretchentragödie mit dem Gretchenlied (Buch), die Formulierung der Aufgabenstellung und der zu bewertende Kommentar (Arbeitsblatt) sowie das Märchen (Tablet). ${ }^{2}$ Insofern steht der Unterricht nun vor der Herausforderung, intertextuelle Bezüge aufzudecken und herzustellen.

Die Lehrerin geht nach der Verteilung der Aufgabe von Gruppe zu Gruppe und begibt sich schließlich zum Tisch der Gruppe „Machandelbaum“:

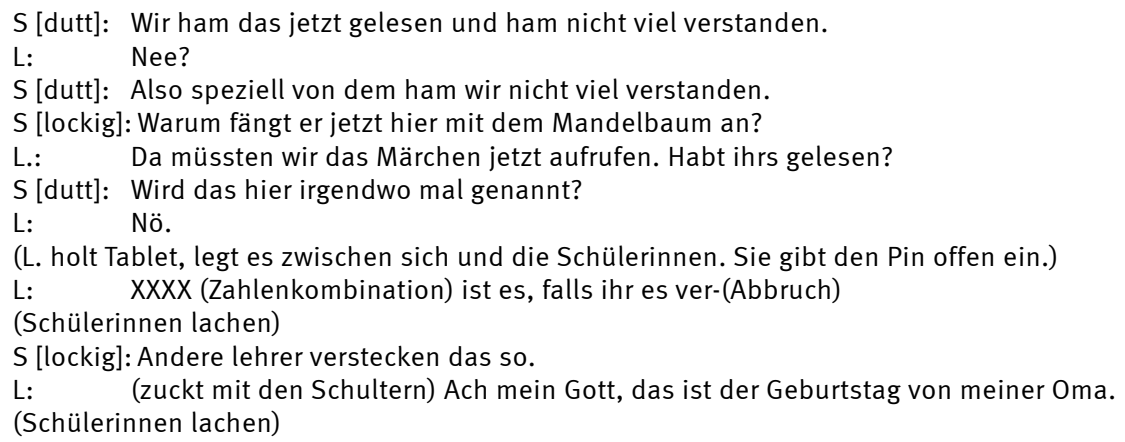

Noch bevor sie richtig angekommen ist, wird ihr von einer Schülerin erklärt, dass die Gruppe „das“ - vermutlich die Aufgabenstellung - gelesen und von „dem“ nicht viel verstanden habe, insbesondere scheint unklar zu sein, was die Aufgabe mit dem „Machandelbaum“ zu tun habe. Damit erklärt sie, dass es trotz ihres Bemühens nicht im Verschulden der Gruppe lag, die Aufgabe noch nicht bearbeitet zu haben und richtet gleichzeitig implizit ein Hilfeersuchen an die Lehrerin. Diese reagiert auf Letzteres und gibt zu verstehen, dass die Arbeitsfähigkeit nur hergestellt werden kann, indem man das Märchen kennt und verbindet damit zugleich die Aufforderung, das Märchen gemeinsam ,aufzurufen“. Der Text wird nicht in einem Buch oder auf einem Arbeitsblatt, sondern mithilfe eines digitalen Mediums (Tablet) bereitgestellt. Durch die digitale Transformation eines traditionell (und eigentlich auch heute noch) in Papierform verfügbaren Textverweises wird die Funktion der kulturellen Überlieferung aktualisiert (vgl. Hickethier 2013: 25) und außerdem eine weitere performative Dimension im Hinblick auf die Handlungsmöglichkeiten und die Rollen von SchülerInnen und LehrerIn erschlossen. Das von den Schülerinnen angesprochene Problem, nämlich die Stellung des Märchens in der Aufgabenbearbeitung, wird von der Lehrerin hier nur teilweise aufgelöst: Sie eröffnet einen technischen, aber wenig inhaltlichen Zugang zur Aufgabe, indem sie keine Interpretation anbietet, sondern das Märchen per Tablet nur verfügbar macht.

2 Im Märchen vom Machandelbaum tötet die neue Frau eines Mannes ihren ungeliebten Stiefsohn und überträgt die Schuld auf die gemeinsame Tochter. Im weiteren Verlauf des Märchens verwandelt sich der Junge in einen Vogel und tötet die Stiefmutter aus Rache. Gretchen nimmt in ihrem Lied „Vom Machandelbaum“ direkt auf dieses Märchen Bezug. 
Interessant ist an dieser Stelle die Tatsache, dass die Lehrerin keine Mittlerfunktion zwischen den verschiedenen Textbezügen zum besseren Verständnis einnimmt. Sie stellt lediglich die Texte zur Verfügung. Es handelt sich also keinesfalls um eine Vereinfachung der Aufgabenbearbeitung, sondern die Schülerinnen werden aufgefordert, eigenständig inhaltliche Bezüge herzustellen. Das Medium Tablet verkörpert an der Stelle zwei Funktionen: Erstens die Mittlerfunktion mit dem Verweis auf das Märchen. Somit wäre auch die Geschichte vom Machandelbaum selbst ein Medium, das wiederum medial vermittelt wird. Zweitens nimmt es eine performative Funktion ein, indem das Tablet selbst symbolisch für einen modernen Unterricht steht, in dem SchülerInnen aufgefordert werden, selbstständig zu lernen. Damit werden die Schülerinnen als technisch versierte Mediennutzerinnen zu ,Entdeckerinnen des Wissens'. Indem die Lehrerin zum einen nicht die vermittelnde Funktion zwischen den Texten einnimmt, sondern dies vollständig in die Verantwortlichkeit der Schülerinnen überträgt, und zum anderen das Tablet als frei zugängliche Informationsquelle inszeniert, zeigt sich eine Autoritätsverschiebung von der klassischen Lehrerinnenrolle in Richtung Medien.

Durch die Nebenerzählung von ihrer Oma verstärkt die Lehrerin die von Offenheit und Zugänglichkeit geprägte Arbeitsatmosphäre. Gleichzeitig stellt dies eine Abkehr von den üblichen in der Schule geltenden Normen dar und signalisiert Nahbarkeit sowie das Nivellieren der Grenzen zwischen den Generationen beziehungsweise der Asymmetrie in der LehrerInnen-SchülerInnen-Beziehung. Im Verlauf des Unterrichts gibt es weitere Nachfragen der Schülerinnen, die die Lehrerin nicht beantwortet, vielmehr verweist sie auf das Märchen als Informationsquelle und reagiert mit stichwortartigen Antworten.

Nach einiger Zeit geht die Lehrerin erneut zur Gruppe „Machandelbaum“, die sich gerade über das Märchen austauscht:

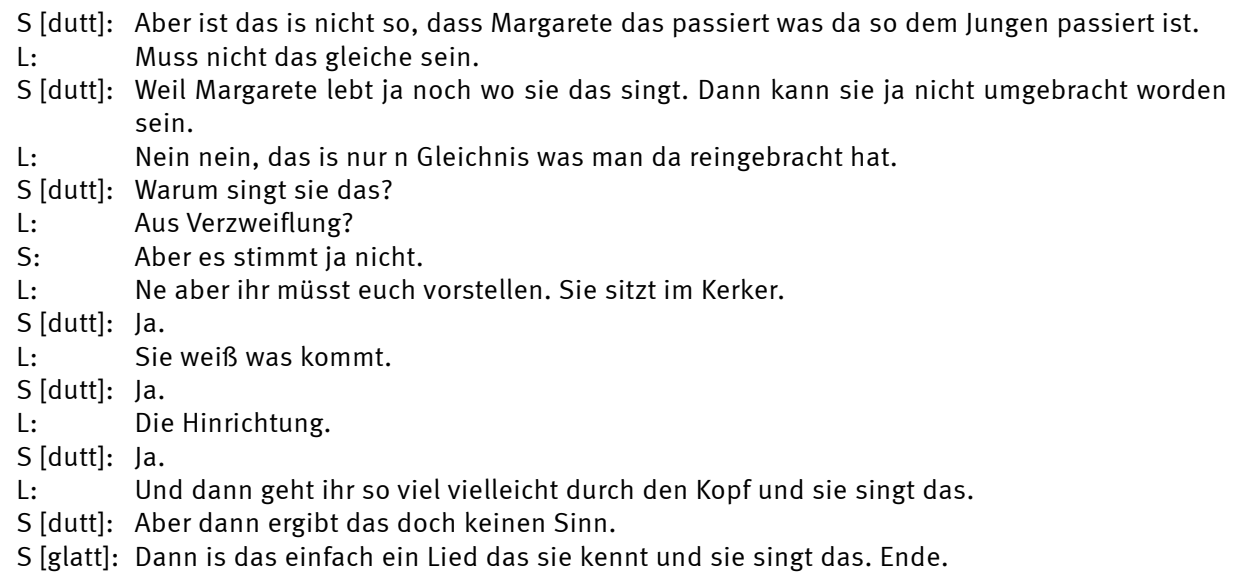

Die Schülerinnen richten inhaltliche Fragen an die Lehrerin, die Schwierigkeiten bei der Trennung der Ebenen Fausttext und Märchen bei der Bearbeitung der Aufgabenstellung erkennen lassen. Drama und Märchen stehen damit aus Sicht der Schülerinnen unvermittelt nebeneinander, der (vermittelnde) Zusammenhang kann (noch) nicht hergestellt 
werden. Die Lehrerin lässt sich auch an dieser Stelle zunächst nicht auf eine inhaltliche Auseinandersetzung ein: Sie beantwortet die Schülerinnenfragen nur knapp und rekurriert auf das Gleichnis als stilistisches Mittel, das „man“ - also eine unbekannte Autorität - „da reingebracht hat". Erst als eine Schülerin fragt, warum Gretchen das Lied singt, bietet die Lehrerin eine inhaltliche Deutung an, die sich an der Perspektive Gretchens orientiert und unter Rückgriff auf eine dramatische Zuspitzung durch die Lehrerin („Sie weiß ja was kommt“ - Pause - „die Hinrichtung“) realisiert wird, jedoch eher an der Oberfläche bleibt und die Frage nach dem Zusammenhang zwischen Gesang und Märchen nicht beantwortet. Die von der Lehrerin angebotene Deutung wird dann auch durch eine der Schülerinnen zurückgewiesen („Das macht doch keinen Sinn“). Eine andere Schülerin drängt jedoch auf einen Abschluss der Diskussion, in dem sie die Deutung des Liedes als stilistisches Mittel akzeptiert und mit dem Aufruf „Ende“ eine rituelle Konklusion vornimmt.

Bei der Betrachtung des Diskurses fällt auf, dass sich auf der Interaktionsebene eine Rahmenkongruenz zeigt: Die Schülerinnen nehmen sich der Verantwortung der Bearbeitung der Aufgabe an und bemühen sich um eine Lösung. Die Redebeiträge sind aufeinander bezogen. Demgegenüber lässt sich aber eine Rahmeninkongruenz auf der inhaltlichen Ebene erkennen, indem die Lehrerin nicht auf die Fragen nach den Zusammenhängen zwischen den Texten eingeht, sondern lediglich auf die Inhalte der einzelnen Texte verweist.

In der Szene bestätigt sich der Orientierungsrahmen, indem die Verschiebung der Expertise von der Lehrerin hin zu den Texten und Medien wiederholt vorgenommen wird. Die Verlagerung der Lehrerinnenexpertise im Modus des gemeinsamen Erkundens wird auch im weiteren Gespräch aufrechterhalten: Die Lehrerin schließt eine inhaltliche Zusammenfassung der Umstände, die zum Tod der Mutter führten, an und berührt dabei weiterhin das Grundproblem der Schülerinnen, nämlich den Zusammenhang zwischen Fausttext und Märchen herzustellen, nicht.

Im Diskurs zwischen der Lehrerin und der Gruppe findet dann eine thematische Verschiebung von der inhaltlichen Fragestellung zum Modus der Ergebnispräsentation statt:

S: $\quad$ Sollen wir die Szene einfach jetzt erzählen, was passiert ist?

L: $\quad$ LJa, was geschieht eigentlich und ist Gretchen dann am Ende gerichtet oder gerettet, das hat nämlich dann noch einen Sinn. Da würde ich dann nochmal mit einsteigen.

S [hinten, sitz.]: Wir sollen jetzt nicht wie das hier steht genau auf das Lied eingehen?

L: $\quad$ LNein [Kopfschütteln]

L: $\quad$ Nein, nein, nein. [schüttelt den Kopf]

$S$ [ebd.]: Also nur erzählen, was passiert.

L: Ja. [nickt]

$\mathrm{S}$ [ebd.]: Ok.

S [Locken]: Also alles was passiert, das könnte ich jetzt erklären und auch das Lied, aber dieser Kommentar.

L:

L: $\quad$ Ne den braucht ihr nicht. [schüttelt den Kopf]

S [Dutt]: Ok. [lachend] 
Eine Schülerin fragt nach den Anforderungen an die Ergebnispräsentation. Die Lehrerin modifiziert mit ihrer Antwort die ursprüngliche Aufgabe: Statt einer Stellungnahme zum literaturwissenschaftlichen Kommentar unter Beachtung des Gretchenliedes und dessen Vergleich mit dem Märchen vom Machandelbaum soll nun lediglich eine einfache Nacherzählung der Szene mit einer abschließenden Bewertung der Situation Gretchens realisiert werden. An dieser Stelle erfolgt eine Einigung zwischen Lehrerin und der Schülerinnengruppe, die auf der Zurücknahme der Einbeziehung bisher ins Feld geführter intertextueller Bezüge basiert. Die Aufgabe wird deutlich reduziert, hin zu einer Fokussierung auf das literarische Werk, den Fauststoff. Das Märchen vom Machandelbaum und der Kommentar, auf den hier erstmals Bezug genommen wird, ist nun nicht mehr Teil der Aufgabenlösung. Die in der Aufgabenstellung geforderte mediale Kopplung zur Erschließung der intertextuellen Verweise scheint nicht zu gelingen und wird von der Lehrerin schließlich aufgegeben.

\section{Zusammenfassung und Fazit}

Dass didaktisch aufbereitetes Material und Medien eingesetzt werden, um den Unterrichtsgegenstand den SchülerInnen im Sinne einer Transformationsleistung näher zu bringen, entspricht sicher dem klassischen Verständnis von Unterricht. Nach Sünkel (1996: 162) gibt es für LehrerInnen die Möglichkeit, sogenannte „,mediale Unterrichtsstücke“ in ihren Unterricht einzubauen. Im vorliegenden Fallbeispiel wird dies über die in SchülerInnengruppen zu bearbeitende Aufgabenstellung realisiert. Der Unterrichtsgegenstand, der in der präsentierten Aufgabenstellung angelegt ist, erweist sich insofern als besonders komplex, als auf unterschiedliche Texte verwiesen wird, die wiederum aufeinander zu beziehen sind.

Wie die oben dargestellte Rekonstruktion zeigt, werden gerade diese intertextuellen Verweisungszusammenhänge zum Verstehensproblem der Schülerinnen, das weder durch ein zusätzliches Medium noch durch die Lehrerin gelöst wird. Nachdem die Schülerinnen Verständnislücken preisgeben, delegiert die Lehrerin die Weitergabe und Vermittlung von Informationen an das Tablet und den darin aufgerufenen Text. Indem sie sich mit einem eigenen Deutungsangebot zurückhält und stattdessen auf das Tablet verweist, weist sie die Verantwortung für Vermittlungsprozesse zurück und gibt diese über weite Strecken an die unterrichtlichen Medien ab.

Im Interaktionsverlauf zeigt sich darin eine Rahmeninkongruenz der beteiligten Akteure: Während die Schülerinnen die Lehrerin als ,Expertin“ adressieren, die inhaltlich Hilfe leisten kann, ebnet sie die hierarchischen Verhältnisse ein, indem sie sich (scheinbar) selber mit auf die Erkundung mittels der Medien begibt. Die Lehrerin, die (eigentlich) einen Vorsprung an Wissen bezüglich der Einordnung der Texte hat, nimmt keine Strukturierung vor, die den Schülerinnen das Lösen der Aufgabenstellung erleichtert.

Aber auch die Medien, auf die die Lehrerin verweist, leisten diese Vermittlung nicht. Den Medien wird eine Funktion als „Boten“ (Krämer 2008: 108ff.) zugesprochen, die sie in diesem Fall für die Schülerinnen offenbar nicht einlösen. Zwar präsentiert beispielsweise das Tablet den Text des Märchens, allerdings wird der fragliche Verweisungszusammenhang für die Schülerinnen dadurch noch nicht wahrnehmbar. Trotzdem 
bleiben die Schülerinnen allein verantwortlich für die Vermittlung der unterschiedlichen Textbezüge, ohne dass die Lehrerin oder ein weiteres Medium als Vermittlungsinstanz fungiert und die Lösung des ,Problems ' beziehungsweise der Aufgabe durch inhaltliche Vorgaben oder eigene Deutungen steuert. Das Verstehensproblem wird schließlich so aufgelöst, dass die Komplexität der ursprünglichen Aufgabe mit Blick auf die anstehende Ergebnispräsentation reduziert wird.

Das Fallbeispiel verweist auf das grundlegende Handlungsproblem von Lehrkräften, die eigene Vermittlungsarbeit zu der in Medien realisierten Vermittlungsarbeit in Beziehung zu setzen. Es zeigt sich, dass das Zusammenspiel zwischen personal-medialer und im weitesten Sinn technisch-medialer Vermittlungsarbeit nicht selbstverständlich ist und intermediale Konfigurationen (vgl. Wirth 2007: 256) konzeptuell besonderer Aufmerksamkeit bedürfen.

\section{Autorenangaben}

\author{
Prof. Dr. Maria Hallitzky \\ maria.hallitzky@uni-leipzig.de \\ Tel.: 0341/ 9731421 (Ansprechpartnerin) \\ Dr. Beate Beyer \\ beate.beyer@uni-leipzig.de \\ Christopher Hempel \\ christopher.hempel@uni-leipzig.de
}

\author{
Johanna Leicht \\ johanna.leicht@uni-leipzig.de \\ Ellen Schroeter \\ eschroet@rz.uni-leipzig.de \\ Universität Leipzig \\ Erziehungswissenschaftliche Fakultät \\ Arbeitsbereich für Allgemeine Didaktik und \\ Schulpädagogik des Sekundarbereichs \\ Dittrichring 5-7 \\ 04109 Leipzig,
}

\section{Literatur}

Berndt, Frauke/Tonger-Erk, Lily (2013): Intertextualität. Eine Einführung. Grundlagen der Germanistik, Bd. 53. Berlin: Erich Schmidt Verlag.

Bohnsack, Ralf (2007): Performativität, Performanz und dokumentarische Methode. In: Wulf, C./Zirfas, J. (Hrsg.): Pädagogik des Performativen. Theorien, Methoden, Perspektiven. Weinheim/Basel: Beltz, S. 200-212.

Bohnsack, Ralf (2014): Rekonstruktive Sozialforschung. Einführung in qualitative Methoden. 9. Aufl. Opladen/Farmington Hills: Barbara Budrich.

Deterding, Sebastian (2013): Mediennutzungssituationen als Rahmungen. Ein Theorieangebot. In: Jandura, O./Fahr, A./Brosius, H.-B. (Hrsg.): Theorieanpassungen in der digitalen Medienwelt. Baden-Baden: Nomos Verlagsgesellschaft, S. 47-69.

Fritzsche, Bettina/Wagner-Willi, Monika (2013): Ethnografie und Videografie in praxeologischer Perspektive. In: Loos, P./Nohl, A.-M./Przyborski, A./Schäffer, B. (Hrsg.): Dokumentarische Methode. Grundlagen - Entwicklungen - Anwendungen. Opladen: Barbara Budrich, S. 268284.

Gruschka, Andreas (2005): Das Kreuz mit der Vermittlung. In: Stadtfeld, P. (Hrsg.): Allgemeine Didaktik im Wandel. Bad Heilbrunn: Julius Klinkhardt, S. 13-37. 
Hallitzky, Maria/Beyer, Beate/Hempel, Christopher/Herfter, Christian/Leicht, Johanna/Saupe, Anja/Schroeter, Ellen (2016): „,...ich weiß nicht was er uns sagen will damit.“ (In-) Transparenz in der Begegnung mit dem Gegenstand am Beispiel des Literaturunterrichts. In: Schude, S./Moegling, K. (Hrsg.): Transparenz im Unterricht und in der Schule. Teil 2. Forschungsergebnisse und Diskussion. Theorie und Praxis der Schulpädagogik, Bd. 33. Immenhausen bei Kassel: Prolog-Verlag, S. 121-145.

Hallitzky, Maria/Beyer, Beate/Hempel, Christopher/Herfter, Christian/Leicht, Johanna/ Schroeter, Ellen (2016): „Was muss'n jetzt nochmal auf den Strich?“. Zur Konstitution von Ergebnisräumen im Literaturunterricht der Sekundarstufe. In: Zeitschrift für interpretative Schul- und Unterrichtsforschung 5(1), 46-62.

Hickethier, Knut (2013): Mediale Wechselwirkungen - Modelle des medialen Zusammenwirkens. In: Höger, I./Oldörp, C./Wimmer, H. (Hrsg.): Mediale Wechselwirkungen. Adaptionen Transformationen - Reinterpretationen. Bonn: Reimer Verlag, S. 13-27.

Hummrich, Merle (2016): Was ist der Fall? Zur Kasuistik in der Erziehungswissenschaft. In: Hummrich, M./Hebenstreit, A./Hinrichsen, M./Meier, M. (Hrsg.): Was ist der Fall? Kasuistik und das Verstehen pädagogischen Handelns. Wiesbaden: Springer VS, S. 13-37.

Kerlen, Dietrich (2003): Einführung in die Medienkunde. Stuttgart: Reclam.

Krämer, Sybille (2004a): Die Heteronomie der Medien. Versuch einer Metaphysik der Medialität im Ausgang einer Reflexion des Boten. In: Journal Phänomenologie, 22, S. 18-38, online: https://drive.google.com/file/d/0B4uR4CFMAGQ3RXBtNHICZ1ZCTE0/view (zuletzt geprüft am 30.03.2017).

Krämer, Sybille (2004b): Was haben ,Performativität" und ,Medialität‘ miteinander zu tun? Plädoyer für eine in der ,Aisthetisierung' gründende Konzeption des Performativen. In: Krämer, S. (Hrsg.): Performativität und Medialität. München: Wilhelm Fink Verlag, S. 13-32.

Krämer, Sybille (2008): Medium, Bote, Übertragung. Kleine Metaphysik der Medialität. Frankfurt am Main: Suhrkamp Verlag.

Martens, Matthias/Petersen, Dorthe/Asbrand, Barbara (2015): Die Materialität von Lernkultur. Methodische Überlegungen zur dokumentarischen Analyse von Unterrichtsvideografien. In: Bohnsack, R./Fritzsche, B./Wagner-Willi, M. (Hrsg.): Dokumentarische Video- und Filminterpretation. Methodologie und Forschungspraxis. Opladen: Barbara Budrich, S. 179-206.

Münker, Stefan (2009): Was ist ein Medium? In: Münker, S. (Hrsg.): Philosophie nach dem „Medial Turn“. Bielefeld: transcript Verlag, S. 39-53.

Nentwig-Gesemann, Iris (2014): Die Unterrichtssequenz Parallelogramme II - Fallrekonstruktion mit der Dokumentarischen Methode. In: Pieper, I./Frei, P./Hauenschild, K./ Schmidt-Thieme, B. (Hrsg.): Was der Fall ist. Beiträge zur Fallarbeit in Bildungsforschung, Lehramtsstudium, Beruf und Ausbildung. Wiesbaden: Springer Fachmedien, S. 123-138.

Pflugmacher, Torsten (2015): Verstehen verstehen - verstehen. Literaturpädagogische Professionalität als Herausforderung von Literaturdidaktik und Literaturvermittlung. In: Bräuer, C./ Wieser, D. (Hrsg.): Lehrende im Blick. Empirische Lehrerforschung in der Deutschdidaktik. Wiesbaden: Springer VS, S. 131-158.

Reh, Sabine/Rabenstein, Kerstin (2013): Die soziale Konstitution des Unterrichts in pädagogischen Praktiken und die Potentiale qualitativer Unterrichtsforschung. Rekonstruktionen des Zeigens und Adressierens. In: Zeitschrift für Pädagogik 59, 3, S. 291-307.

Sünkel, W. (1996): Phänomenologie des Unterrichts. Grundriß der theoretischen Didaktik. Beiträge zur pädagogischen Grundlagenforschung. Weinheim und München: Juventa Verlag.

Tulodziecki, Gerhard/Herzig, Bardo/Grafe, Silke (2010): Medienbildung in Schule und Unterricht. Bad Heilbrunn: Verlag Julius Klinkhardt.

Wirth, Uwe (2007): Intermedialität. In: Anz, T. (Hrsg.): Handbuch Literaturwissenschaft. Gegenstände - Konzepte - Institutionen. Gegenstände und Grundbegriffe, Bd. 1. Stuttgart und Weimar: Verlag J.B. Metzler, S. 254-264. 


\title{
Essbare Dinge in Lehr- und Lernprozessen- Sprachförderung mit Lebensmitteln im Kindergartenalltag
}

\begin{abstract}
Zusammenfassung
Lebensmittel werden in Kindergarten und Schule häufig als didaktische Objekte eingesetzt. Anhand ethnographisch erhobener Daten untersucht der Beitrag aus erziehungswissenschaftlicher und ernährungspädagogischer Perspektive, welche Konsequenzen dies haben kann. Lebensmittel können durch didaktische Transformation ihre ursprüngliche Funktion des Genusses verlieren und Zwänge mit sich bringen. Zugleich wird ihr didaktisches Potenzial in der Sprachförderung bzw. Ernährungsbildung nicht umfassend genutzt.

Schlagwörter: Sprachdidaktik, Ernährungsbildung, ethnographische Forschungsstrategie
\end{abstract}

\section{Edible things in teaching and learning processes - language promotion with food in everyday life in kindergarten}

Foodstuffs are often used in kindergarten and school as pedagogical objects. Drawing on ethnographic data, this article examines the possible consequences this may have from the perspectives of education and nutrition education. Food can lose its original pleasurable function through such pedagogical transformation and bring compulsive behaviour in its wake. At the same time, the pedagogical value of food in language promotion and nutrition education is not fully exploited.

Keywords: Language teaching, nutrition education, ethnographic research strategy

\section{Einleitung}

Der Kindergarten gilt sowohl aus erziehungswissenschaftlicher als auch aus ernährungspädagogischer Sicht als wichtiger Lernort. Dort werden nicht nur die Grundsteine der sprachlichen Bildung gelegt (Neumann 2012), sondern wesentliche Grundlagen für das spätere Ernährungsverhalten entwickelt (Schwartz et al. 2011). Um diese doppelte Bedeutung umfassend erschließen zu können, werden im folgenden Beitrag beide Perspektiven und wissenschaftliche Positionen berücksichtigt.

In den Mittelpunkt des Beitrags soll ein Aspekt gestellt werden, der im Forschungszusammenhang von Erziehung und Ernährung bislang eine eher nachgeordnete Rolle spielt: die Verwendung von Lebensmitteln als „,didaktische Objekte“ in Lehr-Lernprozessen (Neumann 2013: 108; vgl. Röhl 2015). Typischerweise kommen Lebensmittel in Schulfächern wie Arbeitslehre, Biologie, Ernährungsbildung etc. zum Einsatz und sind dort in entsprechende Fachkulturen eingebettet (Bender 2012). Lebensmittel werden jedoch auch in anderen unterrichtlichen Zusammenhängen und Fächern didaktisch genutzt, insbesondere in der sprachlichen Bildung, im Rahmen der kulturellen Bildung oder bspw. sogar in der Mathematik. ${ }^{1}$

1 Die_der Lesende denke an die Tafel Schokolade zur Veranschaulichung des Bruchrechnens. 
Der vorliegende Beitrag bezieht sich auf ethnographische Beobachtungen in einem Schweizer Kindergarten, der von Kindern mit unterschiedlichem Migrationshintergrund besucht wird; Lebensmittel sollen hier die Sprachförderung unterstützen. Für die vorliegende Arbeit erscheint die Verortung des Kindergartens im Schweizer Bildungssystem von Bedeutung: Entsprechend dem aktuellen Volksschulgesetz aus dem Jahr 2005 tritt der obligatorische zweijährige Kindergarten als erste Stufe der elfjährigen Volksschule auf (Kantonsrat 2005: §3). Dementsprechend werden die Kindergärtnerinnen und Kindergärtner als Lehrerinnen und Lehrer bzw. als Lehrpersonen bezeichnet sowie die Sequenzen des organisierten Lehrens und Lernens im Kindergarten als Unterricht. Der Beitrag geht folgender Frage nach: Wie werden Lebensmittel in Lehr-Lernprozesse des Sprachförderunterrichts im Kindergarten einbezogen und welche Herausforderungen können sich dabei ergeben? Dabei interessiert uns vor allem der Umstand, dass Lebensmittel essbar sind bzw. die Option der Essbarkeit als zentrales Merkmal mit sich bringen. Diese Option unterscheidet Lebensmittel von anderen ,Dingen', die im Unterricht verwendet werden.

Nach einem Einblick in den Forschungsstand zum Thema ,Ernährung und Erziehung' und einer kurzen Beschreibung des Forschungsprojekts und der Methode skizzieren wir die curricularen Vorgaben zur Ernährungsbildung und sprachlichen Bildung in vorschulischen Bildungsinstitutionen im Kanton Basel-Landschaft. Danach werden die ausgewählten Szenen unter den Kategorien ,Lebensmittel als didaktische Objekte im Rollenspiel‘ (Feldvignette 1) sowie ,Lebensmittel als didaktische Objekte zur Veranschaulichung' (Feldvignette 2) analysiert bzw. rekonstruiert. In einem letzten Schritt formulieren wir Schlussfolgerungen hinsichtlich des Umgangs mit Lebensmitteln in didaktischen Inszenierungen.

\section{Forschungsstand}

Der Zusammenhang zwischen Ernährung und Erziehung oder Ernährung und Bildung ist in jüngerer Zeit sowohl in der englischsprachigen (z.B. Daniel/Gustafsson 2010; Punch/ McIntosh/Emond 2011; Punch/McIntosh 2014) als auch in der deutschsprachigen Erziehungswissenschaft breit untersucht worden (Althans/Schmidt/Wulf 2015; Althans/Bilstein 2016; Rose/Sturzenhecker 2009; Schütz 2015; Seichter 2012; Täubig 2016). Insbesondere Seichter (2012) legt hinsichtlich der These „Erziehung durch Ernährung“ dar, wie Ernährung in historischen und aktuellen Konzepten u. a. zur moralischen und disziplinarischen Erziehung genutzt wurde und wird. Mehrere erziehungswissenschaftliche Publikationen zum Zusammenhang von Ernährung und Bildung beruhen auf ethnographischen Forschungen (Punch/McIntosh 2014; Schulz 2010a; Schütz 2015). Der Forschungsfokus liegt dabei auf der Frage, wie die Mahlzeit als soziale Situation des gemeinsamen Essens und Trinkens in Bildungsinstitutionen konstituiert und pädagogisch interpretiert wird. Von einigen Forschenden wird dabei auch kritisch dargestellt, wie Erwachsene teilweise regulierend und restriktiv in die Nahrungsaufnahme der Kinder eingreifen, um gesundheitspräventive Ziele durchzusetzen (Punch/McIntosh 2014; Rose 2010).

Ernährungspädagogische Publikationen zeigen teilweise eine etwas andere Perspektive auf die Mahlzeit bzw. auf die Ernährung (Bender 2013; Heindl 2003; Martins 
2006; Methfessel 2015). Hier geht es um die Frage, inwieweit im Rahmen institutioneller Mahlzeiten auch Gestaltungen einer ,Ernährungsbildung' möglich seien (Methfessel 2015). ,Ernährungsbildung' wird dabei abgegrenzt gegenüber einer traditionellen ,Ernährungserziehung', die mit zahlreichen Vorschriften für eine vorgeblich ,richtige Ernährung eher genussfeindliche und teilweise rigide ernährungsbezogene Maßnahmen in Bildungsinstitutionen (und in Familien) hervorbringen kann (Heindl 2003). Diese deutschsprachige kritische Sicht auf diverse ernährungserzieherische Maßnahmen wird unterstützt durch Befunde aus der internationalen ernährungspsychologischen Forschung, die unterschiedliche „Strategien“2 von Erwachsenen und Peers während der Mahlzeiten in privaten und institutionellen Zusammenhängen feststellen und darauf hinweisen, wie ungünstig sich einige auf das Ernährungsverhalten der Kinder in Gegenwart und Zukunft auswirken. Hierzu zählen unter anderem rigide Regulierungen während des Essens, die Verwendung von Lebensmitteln oder Speisen als Bestrafung oder die Anwendung von Zwängen, damit Kinder bestimmte Speisen essen (Martins 2006; Orrell-Valente et al. 2007).

Blickt man jedoch genauer auf den Zusammenhang von Ernährung und Bildung, sind neben einer Ernährungsbildung, die während der Mahlzeiten stattfindet, zusätzlich Fächer wie Ernährungs- und Verbraucherbildung, Biologie, Sachunterricht etc. angesprochen, in denen Lebensmittel zu didaktischen Zwecken zubereitet und gegessen, experimentell untersucht oder aus anderer Perspektive im Unterricht bearbeitet werden. Auch im Kindergarten spielen Lebensmittel aus didaktischer Sicht eine Rolle, etwa zur Sinnesbildung (Meier-Ploeger 2004).

Anders als sonstige „Dinge des Wissens“, wie Wandtafeln oder bestimmte Lehrmittel (Röhl 2013; vgl. Wiesemann/Lange 2015), sind Lebensmittel jedoch zunächst nicht auf didaktische Funktionen ausgerichtet, sondern sollen in erster Linie der Ernährung dienen. Sie sind folglich in vielfältige alltägliche Kontexte eingebettet. Diese Einbettung und Vieldeutigkeit erfordert ,praktische Transformationsleistung[en]“" (Röhl 2015: 162) durch Lehrende und Lernende, um zu bewirken, dass Lebensmittel als didaktische Objekte die beabsichtigten spezifischen didaktischen Funktionen im Unterricht erfüllen können. Die jeweiligen didaktischen Funktionen entspringen zunächst den Planungen der Lehrperson: Im Rahmen des von ihr geplanten und gestalteten Lehrprozesses sollen die Dinge auf ganz bestimmte Weise die anvisierten Lernprozesse der Schülerinnen und Schüler unterstützen. Andere Eigenschaften, welche den alltäglichen Dingen darüber hinaus noch innewohnen und die jenen Planungen nicht dienlich sind, gelten aus Sicht der Lehrperson im Unterricht folglich als überflüssig oder unerwünscht. Gleichzeitig aber, so Röhl (ebd.), ermöglichen gerade die alltäglichen Dinge eine engere Verbindung zwischen ,Welt' und wissenschaftlicher Disziplin. Zudem zeichnen sich Lebensmittel dadurch aus, dass sie mehr oder weniger genussvoll gegessen werden können und eine vielfältige sinnliche Präsenz haben (Morris 2016). Der Umgang mit ihnen ist mit Emotionen (Macht 2008) und teilweise hoher biografischer Bedeutung (Heindl 2003) verbunden. So könnte ihre Nutzung als didaktische Objekte auf Seiten der Lernenden möglicherweise hohe Motivation hervorrufen. Allerdings schließt die didaktische Transformation ein, dass die Lehrperson darüber entscheidet, inwieweit sie der zentralen Option

2 In englischsprachigen Publikationen sind die Begriffe „strategies“ oder „feeding strategies“ gebräuchlich (Martins 2006; Orrell-Valente et al. 2007). 
von Lebensmitteln, der Essbarkeit, im Unterricht Raum geben möchte. Anders als im Kontext von Mahlzeiten innerhalb der Institution Volksschule steht der Verzehr von Lebensmitteln im Unterricht somit prinzipiell zur Disposition, wie aus den folgenden Protokollausschnitten deutlich wird.

\section{Methode}

Die im Beitrag interpretierten Protokollausschnitte sind im Rahmen des Forschungsprojekts MEMOS (Mehrsprachigkeit und Mobilität im Übergang vom Kindergarten in die Primarschule in der Schweiz) der Pädagogischen Hochschule der Fachhochschule Nordwestschweiz verfasst worden. ${ }^{3}$ Das MEMOS-Projekt (2011-2014) wurde als Anschlussprojekt des europäischen Forschungsprojekts HeLiE (Heterogenität und Literalität im Übergang vom Elementar- in den Primarbereich im europäischen Vergleich) der Universität zu Köln ins Leben gerufen und untersuchte den Umgang mit migrationsbedingter Heterogenität und Mehrsprachigkeit in frühkindlichen Bildungsinstitutionen in der Deutschschweiz (Panagiotopoulou/Krompàk 2014; Krompàk 2015; Panagiotopoulou/Kassis 2015). Im Zentrum des MEMOS-Projekts stand die Erforschung des sprachlichen Alltags der Kinder mit Migrationshintergrund. Das Thema ,Lebensmittel' spielte dabei zunächst keine Rolle. Allerdings zeigte eine zweite Analyse des empirischen Materials, dass mehrfach Situationen protokolliert worden waren, in denen Lebensmittel im Zusammenhang mit der unterrichtlichen Sprachförderung verwendet wurden. Die betreffenden Protokollabschnitte spiegelten deutlich die Irritationen der Beobachterin wider. Diese subjektiven Irritationen wurden zum Anlass genommen, das Material aus erziehungswissenschaftlicher und ernährungspädagogischer Perspektive erneut zu untersuchen (Amann/Hirschauer 1997; Goffman 1996; Strauss/Corbin 1996).

Die Interpretation der Protokollabschnitte im vorliegenden Beitrag folgte den methodischen Schritten der Grounded Theory (Charmaz 2006; Strauss/Corbin 1996). Das empirische Material wurde dabei nach Charmaz (2006) in zwei Phasen kodiert: In der ersten Phase erfolgte das streng datenbasierte offene Kodieren (initial coding). In der zweiten Phase, durch das fokussierte Kodieren (focused coding), wurden die offenen Kodes selektiert und die signifikantesten ausgewählt, um sie weiter zu untersuchen. Im Sinne von Strauss und Corbin (1996) sowie Charmaz (2006) wurden die entwickelten Kodes und Kategorien dann verglichen und unter Einbezug von Theorien interpretiert. Im Rahmen des vorliegenden Beitrags ist die Darstellung dieses Verfahrens nur ausschnitthaft möglich.

Im Folgenden werden zwei ausgewählte Protokollabschnitte in Gestalt sogenannter Feldvignetten (Schulz 2010b) wiedergegeben und Kodierungen sowie Analysen vorgenommen (Breidenstein et al. 2015). Um die Vignetten mit Blick auf die Lehrperson angemessen interpretieren zu können, wird vorher der institutionelle Hintergrund skizziert.

3 Am Projekt beteiligte Forscherinnen waren A. Panagiotopoulou, M. Kassis und E. Krompàk. 


\section{Ernährungsbildung und sprachliche Bildung in vorschulischen Bildungsinstitutionen in Basel- Landschaft}

Die beobachteten Situationen stammen aus einem Kindergarten in Basel-Landschaft. In der Schweiz gehört der Kindergarten zur Volksschule. Im Stufenlehrplan (Erziehungsrat des Kantons Basel-Landschaft 1998/2003) sind Stichworte wie ,Ernährung' oder ,Essen' nicht explizit aufgeführt, doch kann die Lehrperson entsprechende Ziele und Themen aufgreifen. Insbesondere in den „Leitideen“ des Lehrplans wird darauf hingewiesen, dass der Kindergarten eine „ganzheitliche Bildung“ ermöglichen und den Kindern unter anderem helfen solle, Anforderungen des täglichen Lebens zunehmend selbstständig zu bewältigen. Der Kindergarten ziele ,auf ein gesteigertes Wohlbefinden der Kinder hinsichtlich Körper, Geist und Seele“ ab (ebd.: 3). Zudem gehe es darum, zu lernen, die eigenen Gefühle und Bedürfnisse wahrzunehmen (ebd.: 8). Auch die sozialen Erfahrungen seien zu unterstützen (ebd.).

Im Vergleich zum Bereich Ernährung stellt die sprachliche Bildung einen expliziten Bestandteil des Stufenlehrplans dar. Im Bildungsbereich Sprache werden Grobziele formuliert, die am Ende des zweiten Kindergartenjahres zu erreichen sind. Darunter finden sich etwa die folgenden: „Wahrnehmen und ausdrücken, beschreiben und erzählen, sprechen und zuhören, sowie verstehen und wiedergeben“ oder „Eigene Empfindungen, Gefühle, Absichten, Wünsche und Bedürfnisse in Worten ausdrücken“ (ebd.: 10). Die Grobziele des Stufenlehrplans sind hierbei nicht themenspezifisch formuliert.

Im Weiteren erscheint wichtig, die Regelungen zu beachten, die den Sprachgebrauch im Kindergarten betreffen. Gemäß Reglement des Bildungsrats Basel-Landschaft von 2009 wird hier ein Gleichgewicht von Standarddeutsch und Schweizerdeutsch angestrebt (Amt für Volksschulen 2007). In dem im Kanton Basel-Landschaft verwendeten Kindergartenlehrmittel LEZUS (Aellig/Alt 2006) wird allerdings, gestützt auf empirische Studien über den unterschiedlichen Erfolg dieser beiden Modalitäten des Sprechens für die Sprachförderung, für einen konsequenten Gebrauch der Standardsprache plädiert (ebd.: 42).

Zusammenfassend kann festgestellt werden, dass die Bildungsbereiche Ernährung und Sprache im Curriculum Basel-Landschaft implizit (Ernährung) und explizit (Sprache) vorkommen. Während die beiden Bereiche auf curricularer Ebene nicht miteinander verknüpft sind, konnten in der ethnographischen Studie viele Situationen rekonstruiert werden, in denen das Thema Ernährung mit Zielen sprachlichen Lernens verbunden wurde. Das folgende Kapitel zeigt dies anhand von Lehr-Lernprozessen rund um Maroni und Karottensaft. 


\section{Ergebnisse}

\section{1 „Heiße Maroni, Maroni von Toni!“ - Lebensmittel als didaktische Objekte im Rollenspiel}

\section{Feldvignette 1: ,Maroni“}

Die Lehrperson bereitet mit einem Kind den Maroniofen vor, in dem Maroni zubereitet werden. Die anderen Kinder spielen währenddessen mit der Geisterbahn oder mit den Spielzeugen aus der Herbstmesse. Es riecht nach Maroni. Die Kinder kommen in den Kreis. Einige von ihnen fangen an, ein Lied über Maroni zu singen. „Ihr habt es gesehen, aber habt ihr nichts gerochen?“, fragt die Lehrperson. „Ich habe gericht“, sagt Erkan. „Gerochen“, korrigiert die Lehrperson. „Auf Schweizerdeutsch heißt es ,geschmöckt““, ergänzt sie. Dann singen Kinder und Lehrperson zusammen das Lied über Maroni. „Wenn man Maroni kauft, darf man sie nicht mit bloßen Händen anfassen. Bekommt ihr es in die Hände?“, fragt die Lehrperson. „Nein, in eine Tüte“, antwortet ein Kind. Die Lehrperson zeigt den Kindern, wie man eine Tüte aus Zeitungspapier faltet. Als die Papiertüten fertig sind, singen die Kinder nochmals das Lied. Die Lehrperson nimmt einen Hut und ruft laut: „Heiße Maroni, Maroni von Toni!“ Sie spielt die Rolle eines Maroniverkäufers. Die Kinder kommen zu ihr und kaufen von ihr die inzwischen heißen Maroni. „Guten Tag, was wünschen Sie?“, fragt der ,Maroniverkäufer“. „Hundert Gramm Maroni“, sagt Flamur. „Vier Franken. Bitteschön“, antwortet der ,Maroniverkäufer‘. Die Kinder kommen einzeln zum ,Maroniverkäufer‘ und sagen, wie viel Gramm Maroni sie möchten, und sie bezahlen vier Franken, indem sie viermal in die Hand der Lehrperson schlagen. Ich werde in's Spiel einbezogen und werde aufgefordert zum ,Maroniverkäufer‘ zu gehen, um Maroni zu kaufen. Am Ende des Spiels singen die Kinder das ,Guten-Appetit-Lied“ und gehen in die Garderobe, um die Maroni zu essen (Protokoll vom 1.11.2011, Kindergarten, Krompàk).

Die im Text wiedergegebene didaktische Inszenierung ist jahreszeitlich eng mit der Basler Herbstmesse ${ }^{5}$ verbunden. Das Lebensmittel Maroni, die Esskastanie, die es vor allem (aber nicht nur) in Herbst und Winter auf den Jahrmärkten zu kaufen gibt, wird in vielfältiger und aufwändiger Weise, insbesondere in einem Rollenspiel, didaktisch genutzt. Dabei werden sinnliche Erlebnisse angeboten, die mit einer doppelten Intervention der Lehrperson zur Sprachförderung verknüpft sind. Hierbei dient das Bilden des Partizip Perfekts des Verbs ,riechen“ innerhalb der Standardsprache nicht nur grammatikalischen Zielen, sondern wird verbunden mit einer dialektalen Ergänzung und der Pflege des Dialekts. Bei dem in der Situation genutzten Maroni-Lied handelt sich um ein bekanntes Kinderlied auf Schweizerdeutsch. ${ }^{6}$ Die didaktische Inszenierung endet, indem die Kinder mit einem anderen Lied das Ritual zur Einleitung der Znüni-Mahlzeit ${ }^{7}$ vollziehen, dabei verlassen sie das Lern- und Spielzimmer und essen in der Garderobe ihre ,selbstgekauften` Maroni.

In der beschriebenen didaktischen Inszenierung stehen die Maroni im Mittelpunkt. Schaut man nur auf die inszenierte Kaufsituation, würde sich das Lebensmittel prinzipiell durch andere Gegenstände ersetzen lassen. Innerhalb der didaktischen Gesamtkomposition, die auf einen Besuch der Herbstmesse folgt und auf diesen jahreszeitlichen kulturellen Kontext abgestimmt ist, wäre es jedoch nicht austauschbar. Durch die In-

4 Die Namen der Teilnehmenden sind anonymisiert.

5 Die Herbstmesse in Basel wird in den Bildungsinstitutionen mit den Kindern besucht.

6 Es handelt sich um ein Lied von A. Bond (siehe: http://andrewbond.ch). Zur Verdeutlichung sei die erste Strophe des Liedes hier zitiert: „Marini, Maruni, Maroni, die chaufed mer immer bim Toni, er schnidt en Schlitz und denn chunt dHitz, Marini, Maruni, Maroni“. Der „Znüni“ ist die Zwischenmahlzeit am Vormittag. 
szenierung eines kulturell geprägten Kontextes und die aktive Partizipation der Kinder werden hier Prozesse der sozialen Reproduktion (Bourdieu 1977; Corsaro 2005) sowie die Aneignung von kulturellen Werten (Mead 1934) sichtbar. Obwohl die Maroni zubereitet und ,gekauft' werden, endet die aufwändige Inszenierung vor ihrem Verzehr. Dies irritiert, denn angesichts der Vielfalt (Zubereitung, Singen, Basteln, Rollenspiel) der didaktischen Inszenierung und der damit verbundenen Alltagsnähe wäre das Essen der Lebensmittel zu erwarten gewesen: Nach dem Erwerb der Maroni werden sie üblicherweise warm gegessen. Der Verzehr der Esskastanien findet jedoch außerhalb des organisierten Lehr-Lernprozesses in der Garderobe statt. Die räumliche Abgrenzung deutet an, dass die Verzehrsituation zwar pädagogisch-ritualisiert eingeleitet, aber ansonsten von der Lehrperson nicht als didaktische Aufgabe angesehen wird. Die zentrale Option des Lebensmittels, seine Essbarkeit, bleibt aus dem Unterricht ausgeklammert.

Indem die Lehrperson den Verzehr der Maroni in der Garderobe lokalisiert, verhindert sie in pragmatischer Weise eine Verschmutzung des Lern- und Spielzimmers. Beim Essen der Maroni könnten Krümel und Schalenreste auf den Boden fallen. Das Vorgehen entspricht verbreiteten Regelungen an der Volksschule, die den Verzehr von Lebensmitteln während des Unterrichts verbieten (höchstens das Trinken ist gestattet). Die körperliche Disziplinierung im schulischen Unterricht (Foucault 1977) schließt also zumeist ein Essverbot ein, sodass das Essen während des Unterrichts von Lernenden allenfalls heimlich durchgeführt wird und nur in den Pausen offiziell erlaubt ist. Zugleich sind für die schulischen Zwischenmahlzeiten der jüngeren Kinder im Allgemeinen die Eltern verantwortlich. Lehrpersonen gelten als für den Unterricht zuständig - und nicht für die Beköstigung und körperliche Versorgung der Kinder. Die Garderobe als Ort für die Zwischenmahlzeit ist somit als räumlicher Übergangsbereich zu deuten, der symbolisiert, dass die Zuständigkeit des Kindergartens an diesem Ort (fast) endet: Hier löst zum einen die Pause oder die Freizeit den Unterricht ab und es beginnt zum anderen die Verantwortung der Eltern (Jäger/Biffi/Halfhide 2006). Der Verzehr der Lebensmittel in der Garderobe markiert diese doppelte ,Grenze' (zwischen Unterricht und Pause/ Freizeit und zwischen den Zuständigkeitsbereichen), und die Lehrperson folgt dieser Grenzziehung (Fritzsche/Rabenstein 2009).

\section{2 „Nase zu und runter damit“ - Lebensmittel als didaktische Objekte zur Veranschaulichung}

\section{Feldvignette 2: ,Karottensaft"}

Die Kinder sitzen im Kreis und die Lehrperson zeigt ihnen verschiedene Bilder, mit deren Hilfe die Kinder zusammengesetzte Wörter bilden sollen. Nun kommt das Bild vom Karottensaft und die Lehrperson fragt die Kinder: „Hat jemand von euch schon Karottensaft getrunken?“ „Nein!“, antworten die Kinder im Chor. „Dann holt mal eure Becher“, gibt die Lehrperson den Kindern die Anweisung. Alle Kinder bekommen ein wenig Karottensaft in ihren Becher. Sie vergleichen die Farbe des Karottensaftes mit der Farbe des abgebildeten Karottensaftes auf der Wortkarte. Alle Kinder probieren den Karottensaft, nur Flamur weigert sich davon zu trinken. „Pfui!“, sagt Flamur. „Das ist nicht Pfui“, mahnt ihn Aara. „Das ist fein!“ „Pfui!“, sagt Flamur nochmals. „Du trinkst das Ganze, ich habe dir ganz wenig gegeben“, sagt die Lehrperson zu Flamur. Dann wendet sie sich zu mir und erklärt: „Flamur ist ganz heikel.“ „Nase zu und runter damit“, ermuntert die Lehrperson Flamur. „Apfelsaft gibt erst, wenn man den Karottensaft getrunken hat.“ „Mach die Nase zu!“, rufen die Kinder. Flamur weigert sich immer noch, den Karottensaft zu trinken. Er versteckt sein Gesicht in seinem Pullover. 
„Karottensaft ist so lecker“, sagt Aara. „Du musst noch trinken“, sagt die Lehrperson. „Ich trinke es niemals!“, ruft Flamur. „Flamur, wenn du es trinkst, ist es schon fertig“, ermutigt ihn Tobias. Die Kinder bekommen kleine Putzschwämme, um die Becher am Waschbecken zu putzen. Flamur hat den Karottensaft doch nicht getrunken. Er darf aber seinen Becher mit dem Schwamm putzen. Luana fragt ihn beim Abwaschen der Becher: „Hast du den Karottensaft getrunken?“ „Nein“, antwortet Flamur. Luana ruft gleich die Lehrperson: „Frau B., Frau B., Flamur hat den Karottensaft nicht getrunken!“ „Ich weiß“, antwortet die Lehrperson und fügt noch hinzu: „Er ist ganz heikel“ (Protokoll vom 31.1. 2012, Kindergarten, Krompàk).

Um den Kindern das zusammengesetzte Wort ,Karottensaft' nicht nur visuell und akustisch nahezubringen, sondern auch olfaktorisch und gustatorisch, hat die Lehrperson Saft dabei. Ziel ist wahrscheinlich die umfassende sinnliche Veranschaulichung des zu erlernenden Wortes. Nachdem die Lehrperson den Saft verteilt hat, stößt sie auf den Widerstand von Flamur, der den reibungslosen Verlauf der didaktischen Inszenierung stört. Die Lehrperson setzt anschließend mehrere Strategien ein, um Flamur dazu zu bewegen, den Saft zu kosten. Dabei wird sie von den anderen Kindern unterstützt, die diese zum Teil wiederholen und verstärken oder durch andere Strategien ergänzen.

Zunächst übt die Lehrperson Druck auf den Jungen aus, sie versucht ihn zugleich zu überzeugen, indem sie darauf verweist, dass die zu verzehrende Menge sehr gering sei. Das Quantitätsargument erinnert an die unabdingbare, aber unangenehme Einnahme einer Arznei, die ja auch zumeist in geringen Mengen verzehrt werden muss. Flamur wird von der Lehrperson hier zum ersten Mal in der Situation als ,ganz heikel“ bezeichnet. Später kommt diese Formulierung erneut vor. Im Schweizerdeutschen wird das Wort ,heikel' synonym zu ,empfindlich` verwendet; weiterhin bleibt hier aber seine konkrete Bedeutung offen. Die Bezeichnung, heikel sein' kann einerseits auf eine mögliche Erklärung des kindlichen Verhaltens gegenüber der Beobachterin hindeuten, andererseits stellt der Status einen gewissen Schutz für Flamur dar. Durch das Adverb ,ganz' wird das ,Heikel-Sein' verstärkt und Flamur noch mehr in einen Sonderstatus gerückt: Ein ,heikles' Kind fordert behutsame und vorsichtige Behandlungen sowie entsprechende Anpassungen des eigenen Verhaltens, was die Lehrerin zuletzt auch tut.

In der zweiten Strategie der Lehrperson kommt der konstruierte Arznei-Charakter des Saftes noch deutlicher zum Ausdruck. Das Riechen soll ausgeschaltet werden, um den Saft zu schlucken. Dieser Trick funktioniert bei Nahrungsmitteln, bei denen der Geruchssinn den Geschmackssinn ergänzt und verstärkt. Die verschlossene Nase soll Flamur also helfen, die Sinneswahrnehmung zu minimieren und die Nahrungsaufnahme schnell hinter sich zu bringen.

Dann wird die dritte Strategie, das Versprechen einer Belohnung bzw. die Verweigerung einer möglichen Alternative eingesetzt: „Apfelsaft gibt erst, wenn man den Karottensaft getrunken hat.“ Diese Strategie erinnert an die außerschulische ernährungserzieherische Strategie „Dessert gibt es erst nach dem Essen“ (Orrell-Valente et al. 2007). Flamurs Reaktionen jedoch zeigen weiterhin heftige Ablehnung und sogar Ekel gegenüber dem Saft. Aus ernährungspädagogischer Sicht ist diese Ablehnung wenig erstaunlich: Gerade Kinder im Kindergartenalter entwickeln häufig Neophobien, also starke Abneigungen gegenüber unbekannten Lebensmitteln (Martins 2006), und der Ekel vor einem Lebensmittel hängt nicht von der zu verzehrenden Menge ab (Rozin/ Fallon 1987). Flamurs Verweigerung äußert sich in zunehmend deutlichen Reaktionen, zuerst mit Worten, einem mehrmaligen „Pfui“, dann mit der Körperhaltung. Die Geste 
des ,Sich-Versteckens' unter dem Pullover kann als Scham in Form von Schutzsuche interpretiert werden. Im Sinne von Magyar-Haas (2011) weist die Körperposition von Flamur auf ein ,sich abgrenzen, verstecken, distanzieren Wollen“ (Magyar-Haas 2011: 284) und folglich auf eine für ihn beschämende Situation hin. Doch obwohl der Druck durch die Lehrerin eskaliert („Du musst noch trinken“), bleibt Flamur standhaft und formuliert schließlich seine felsenfeste Überzeugung: „Ich trinke es niemals.“ Während der gesamten Situation wird der Druck, den die Lehrperson ausübt, von den anderen Kindern verstärkt. Sie zeigen zwar auch Solidarität, wie Tobias, treten aber zugleich als Hüter_innen der Autorität der Lehrperson auf. Letztlich verdankt Flamur es seinem Sonderstatus des ,Heikel-Seins', dass er den Karottensaft nicht trinken muss. Während der ganzen Szene wird weder von der Lehrperson noch von den Kindern nach dem Grund gefragt, warum Flamur den Karottensaft nicht trinken möchte.

Die Lehrperson realisiert im Rahmen der Sprachförderung hier eine didaktische Inszenierung, in der die Kinder, überwiegend Kinder mit Migrationshintergrund, zusammengesetzte Wörter kennenlernen. Dabei orientiert sie sich am Lehrmittel LEZUS von Aellig und Alt (2009), in dem verschiedene Übungen zur Wortzusammensetzung angeboten $\operatorname{sind}^{8}$; u. a. wird das Lebensmittel Karottensaft mit dem Ziel der Veranschaulichung von ,Karotte“ und ,Saft‘ verwendet. Im Zuge der Auseinandersetzung mit Flamur verliert die Lehrperson das Ziel der Sprachförderung jedoch aus den Augen und nutzt ernährungserzieherische Aktionen (im obigen normativen Sinn) und „Ernährung zur Disziplinierung“ (Seichter 2012), um das Kind zum Trinken zu bewegen. Die Lehrperson hält daran fest, dass Flamur den Saft kosten muss und versucht nicht, die Sprachförderung auf andere Weise zu realisieren. Auch die ursprüngliche didaktische Funktion der Veranschaulichung durch ein umfassendes Angebot von Sinneseindrücken wird mit der Strategie des ,Nase-Zuhaltens“ beim Trinken bei Flamur aufgegeben.

Die Störung des didaktischen Ablaufs geht damit einher, dass die Lehrperson das Einverleiben des Saftes erzwingen möchte. Hier endet jedoch ihre didaktische Macht. Letztlich versucht sie, eine Form der Kontrolle über den Körper des Kindes zu erlangen, die über das übliche Maß in Unterrichtssituationen (,Stillsitzen-Müssen', ,Nicht-Sprechen-Dürfen“ etc.) hinausgeht (Foucault 1977) und den Schüler als „ganze Person“ (Kolbe et al. 2009: 155) erfassen möchte. Wird die Macht über den Körper, in diesem Fall das Trinken des Karottensaftes, forciert, kann es zu Scham, Ekel und Verweigerung auf Seiten der Kinder führen. Die Lehrperson beendet diesen Versuch, indem sie darauf verweist, dass Flamur ein „heikles Kind“ sei.

Bei der Lehrperson und den Kindern sind zudem Praktiken zu rekonstruieren, die aus dem alltäglichen Umgang zwischen Erwachsenen und Heranwachsenden bei Mahlzeiten stammen (Orrell-Valente et al. 2007; Martins 2006). Außerunterrichtliche Praktiken sickern somit im Umgang mit dem Essen in den Unterricht ein (Rabenstein/ Steinwand 2012: 69). Zugleich sind im Kontext von Flamurs Weigerung auf Seiten der Lehrperson keine „Lehrerpraktiken“ (Bennewitz 2014) zu rekonstruieren, die sich im Sinne des Kindergartencurriculums und der Sprachförderung anbieten würden: etwa eine Aufforderung an Flamur, von möglichen Erfahrungen mit dem Karottensaft zu er-

8 „Wer den Aufwand nicht scheut, kann zudem mit der Klasse aus Orangen selber Saft pressen, eine Erfahrung, die heute nicht mehr alle Kinder kennen. Eine Saft- (oder Frucht-) Blinddegustation wäre eine beliebte Ergänzung oder Alternative“ (Aellig/Alt 2009: 110). 
zählen oder seine Abneigung zu verbalisieren. Die durch die Lehrperson beabsichtigte didaktische Transformation des Lebensmittels bleibt in diesem Fall unvollständig.

\section{Lebensmittel als didaktische Objekte}

Die Interpretationen der beiden Situationen aus dem Kindergarten spiegeln die Nutzung von Lebensmitteln in zwei vielseitigen unterrichtlichen Inszenierungen wider. Die Lebensmittel werden als didaktische Objekte zur Sprachförderung eingesetzt, einerseits bei einem Rollenspiel (Feldvignette 1), andererseits zur sinnlichen Veranschaulichung (Feldvignette 2). Ihr zentrales Merkmal, die Essbarkeit, ist dabei in unterschiedlicher Weise mit der didaktischen Funktion verbunden: Im Rahmen der Situation ,Maroni klammert die Lehrperson den Verzehr der Esskastanien aus dem Unterricht konsequent aus und kann störungsfrei didaktisch agieren. Hingegen möchte die Lehrperson im Rahmen der Situation ,Karottensaft‘ die Essbarkeit des Lebensmittels didaktisch nutzen und wird mit nicht geplanten Irritationen konfrontiert.

Als essbare Dinge des Alltags bringen die Lebensmittel die Option des Genusses mit sich, die jedoch eigentlich nicht zum schulischen Unterricht gehört und dort nur ausnahmsweise erlaubt ist. Die Rolle der_des Schüler_in impliziert üblicherweise nicht, dass sie_er sich im Unterricht ein bestimmtes Lebensmittel einverleiben muss - ein Anspruch, der auch außerhalb des Unterrichts bei schulischen Mahlzeitensituationen für disziplinarische Irritationen sorgen kann, wie die eingangs skizzierten Forschungen zeigen. Essen im Unterricht überschreitet strukturell gesetzte Grenzen in der Volksschule.

Für Lernende werden die Lebensmittel durch das Verbot, das in der Grenzüberschreitung liegt, ebenso wie durch das Genussversprechen (sofern sie die Lebensmittel gerne verzehren würden) einerseits zu emotional anregenden didaktischen Objekten - andererseits können die Lebensmittel dazu führen, dass Kinder und Jugendliche als „ganze Personen“ (Kolbe et al. 2009: 155) unter didaktischen Druck geraten, wenn die Lehrperson ihnen zumutet, ein Lebensmittel zu verzehren und somit die Grenzen der Schülerrolle nicht respektiert.

Aus Sicht der Lehrenden bergen die Lebensmittel, mehr noch als andere Dinge, die üblicherweise nicht in den Unterricht gehören, didaktische Ambivalenzen: Sie verbinden Schule und Alltag und gerade ihr Genussversprechen könnte hochmotivierend wirken. Essen gilt als ,anständige Lust“ (Zischka/Ottomeyer/Bäumler 1993). Diese wäre im Unterricht aus institutioneller Sicht prinzipiell erlaubt - verursacht aber möglicherweise Unruhe auf Seiten der Lernenden oder Schmutz. Lebensmittel als essbare Dinge könnten sich im Unterricht somit als „,nicht gefügig erweisen“ (Röhl 2013: 151). Wollte eine Lehrperson dies vermeiden, wie anhand der ersten Feldvignette interpretiert, würde sie die zentrale Option der Lebensmittel, die Essbarkeit, im Zuge der Transformation in didaktische Objekte ,wegdidaktisieren'. Sie würde damit aber auch gezielt auf die didaktischen Angebote verzichten, die sich gerade durch die Essbarkeit eröffnen könnten.

Dass Lebensmittel im Alltag insbesondere gegessen werden, beeinflusst ihre didaktische Objektivierung darüber hinaus auch deshalb, weil der Verzehr nicht nur für die Lernenden, sondern ebenso für die Lehrenden mit außerunterrichtlichen Essstrategien verbunden ist. Unterrichtsstörungen im Kontext von alltäglichen Dingen sind nichts Unge- 
wöhnliches (Röhl 2013). Üblicherweise jedoch stellen die Lehrenden die unterrichtliche Ordnung wieder her. Im vorliegenden Fall sind allerdings mahlzeitenbezogene außerunterrichtliche Praktiken so dominant, dass die beabsichtigte didaktische Objektivierung der Lebensmittel im Dienste der Sprachförderung kurzzeitig nicht mehr vollzogen wird.

Die Interpretationen zu den Feldvignetten zeigen somit, dass sich Lebensmittel als Dinge des Alltags in didaktischen Zusammenhängen vielseitig inszenieren lassen - dabei aber möglicherweise spezifische Widerstände zeigen, die mit ihrer typischen Option des Verzehrs zusammenhängen. Sowohl aus dem ,Essen-Dürfen“ als auch aus dem ,Nicht-Essen-Müssen“ ließe sich mit Blick auf beide Szenen didaktischer Gewinn im Sinne curricularer Vorgaben generieren, wobei im Zuge der didaktischen Transformation jedoch jeweils mit Herausforderungen zu rechnen wäre. Die Essbarkeit von Lebensmitteln stellt in Lehr-Lernprozessen somit eine Option dar, welche den didaktischen Umgang mit ihnen erheblich beeinflusst.

\section{Autorenangaben}

Prof. Dr. Ute Bender

Pädagogische Hochschule FHNW, Basel

Institut Sekundarstufe I und II

Professur für Gesundheit und Hauswirtschaft

ute.bender@fhnw.ch
Dr. phil. Edina Krompàk

Pädagogische Hochschule FHNW, Basel

Institut Sekundarstufe I und II

Professur für Professionsentwicklung

edina.krompak@fhnw.ch

\section{Literatur}

Aellig, Steff/Alt, Elsbeth (2009): LEZUS - Von der Lauterfassung zur Schrift. Jahresprogramm zur Sprachförderung in Vorschule und Kindergarten. Handbuch für Lehrpersonen und Projektleitungen. 4. Aufl. Bern: Schulverlag.

Althans, Birgit/Bilstein, Johannes (Hrsg.) (2016): Essen - Bildung - Konsum. Wiesbaden: Springer.

Althans, Birgit/Schmidt, Friederike/Wulf, Christoph (Hrsg.) (2014): Nahrung als Bildung. Interdisziplinäre Perspektiven auf einen anthropologischen Zusammenhang. Weinheim u. a: Beltz Juventa.

Amann, Klaus/Hirschauer, Stefan (1997): Die Befremdung der eigenen Kultur. Ein Programm. In: Hirschauer, S./Amann, K. (Hrsg.): Die Befremdung der eigenen Kultur. Zur ethnographischen Herausforderung soziologischer Empirie. Frankfurt a. M.: Suhrkamp, S. 7-52.

Amt für Volksschulen (2007): Reglement zur Gebrauch der deutschen Standardsprache. Liestal: Bildungs-, Kultur- und Sportdirektion Basel-Landschaft. Amt für Volksschulen [http:// edudoc.ch/record/33422/files/reglement_standardsprache.pdf], Zugriff am 23.12.16.

Bender, Ute (2012): Aufgabenkulturen in der Konsumbildung entwickeln. In: Haushalt in Bildung \& Forschung 1, 3, S. 77-88.

Bender, Ute (2013): Fachdidaktik Ernährungs- und Konsumbildung. In: Bender, U. (Hrsg.): Ernährungs- und Konsumbildung. Perspektiven und Praxisbeispiele für den Hauswirtschaftsunterricht. Bern: Schulverlag plus AG, S.13-20.

Bennewitz, Hedda (2014): „Doing Teacher“ - Forschung zum Lehrerberuf in kulturtheoretischer Perspektive. In: Terhart, E./Bennewitz, H./Rothland, M. (Hrsg.): Handbuch der Forschung zum Lehrerberuf. 2. überarb. u. erw. Aufl. Münster u. a.: Waxmann, S. 262-284. 
Bourdieu, Pierre (1977): Outline of a theory of practice. Cambridge: Cambridge University Press. Breidenstein, Georg/Hirschauer, Stefan/Kalthoff, Herbert/Nieswand, Boris (2015): Ethnografie. Die Praxis der Feldforschung. 2. überarb. Aufl. Konstanz: UVK.

Charmaz, Kathy (2006): Constructing Grounded Theory. A Practical Guide Through Qualitative Analysis. London u. a: Sage.

Corsaro, William A./Molinari, Luisa (2005): I Compagni. Understanding Children`s Transition from Preschool to Elementary School. New York/London: Teachers College Press.

Daniel Paul/Gustafsson Ulla (2010): School lunches: Children's services or children's spaces? In: Children's Geographies 8, 3, S. 265-274.

Erziehungsrat des Kantons Basel-Landschaft (1998/2003): Stufenlehrplan Kindergarten Kanton Basel-Landschaft. Ergänzt im Bildungsbereich Sprache gem. Beschluss des Erziehungsrats vom 26. Februar 2003. Liestal: Erziehungsrat. [www.sprachenunterricht.ch/sites/default/ files/Stufenlehrplan\%20Kindergarten\%20Kanton\%20Basel-Landschaft.pdf], Zugriff am 23.12.16.

Foucault, Michel (1977): Überwachen und Strafen. Die Geburt des Gefängnisses. Frankfurt a. M.: Suhrkamp.

Fritzsche, Bettina/Rabenstein, Kerstin (2009): „Häusliches Elend“ und „Familienersatz“: Symbolische Konstruktionen in Legitimationsdiskursen von Ganztagsschulen in der Gegenwart. In: Ecarius, J./Groppe, C./Malmede, H. (Hrsg.): Familie und öffentliche Erziehung. Theoretische Konzeptionen, historische und aktuelle Analysen. Wiesbaden: Verlag für Sozialwissenschaften, S. 183-200.

Goffman, Erving (1996): Über Feldforschung. In: Knoblauch, H. (Hrsg.): Kommunikative Lebenswelten. Zur Ethnographie einer geschwätzigen Gesellschaft. Konstanz: UVK, S. 261-269.

Heindl, Ines (2003): Studienbuch Ernährungsbildung. Ein europäisches Konzept zur schulischen Gesundheitsförderung. Bad Heilbrunn: Klinkhardt.

Jäger, Marianna/Biffi, Cornelia/Halfhide, Therese (2006): Schlussbericht. Grundstufe als Zusammenführung zweier Kulturen - Teil 1. Eine Ethnographie des Kindergartens. Pädagogische Hochschule Zürich. [https://phzh.ch/MAPortrait_Data/53613/8/A\%20Schlussbericht\%20 Alltagskultur_Erster\%20Schultag_CB_MJ_ohne\%20Kap.6.pdf], Zugriff am 23.12.16.

Kantonsrat (2005): Volksschulgesetz (VSG) [http://www2.zhlex.zh.ch/appl/zhlex_r.nsf/0/B6DFC 1347AA5482FC12575C1003D4B7F/\$file/412.100_7.2.05_65.pdf], Zugriff am 16.12.2016.

Kassis-Filippakou, Maria/Panagiotopoulou, Argyro (2015): Sprachförderpraxis unter den Bedingungen der Diglossie - Zur „Sprachentrennung“ bzw. „Sprachenmischung“ als Normalität im Kindergartenalltag der deutschsprachigen Schweiz. In: Schweizerische Zeitschrift für Bildungswissenschaften 37, 1, S. 113-129.

Kolbe, Fritz-Ulrich/Reh, Sabine/Idel, Till-Sebastian/Fritzsche, Bettina/Rabenstein, Kerstin (2009): Grenzverschiebungen des Schulischen im Ganztag - Einleitung zur schultheoretischen Diskussion. In: Kolbe, F.-U./Reh, S./Idel, T.-S./Fritzsche, B./Rabenstein, K. (Hrsg.): Ganztagsschule als symbolische Konstruktion. Fallanalysen zu Legitimationsdiskursen in schultheoretischer Perspektive. Wiesbaden: Verlag für Sozialwissenschaften, S. 151-157.

Krompàk, Edina (2015): Sprachliche Realität im Schweizer Kindergarten- und Schulalltag. CodeSwitching und Sprachentrennung bei mehrsprachigen Kindern. In: Schnitzer, A./Mörgen, R. (Hrsg.): Mehrsprachigkeit und (Un)gesagtes. Sprache als soziale Praxis im Kontext von Heterogenität, Differenz und Ungleichheit. Weinheim u. a.: Beltz Juventa, S. 175-193.

Macht, Michael (2008): How emotions affect eating: A five-way model. In: Appetite 50, 1, S. 1-11. Magyar-Haas, Veronika (2011): Subtile Anlässe von Scham und Beschämung in (sozial)pädagogischen Situationen. In: Arbeitskreis Jugendhilfe im Wandel (Hrsg.): Jugendhilfeforschung. Kontroversen - Transformationen - Adressierungen. Wiesbaden: Verlag für Sozialwissenschaften, S. 277-289. 
Martins, Yolanda (2006): Dietary Experiences and Food Acceptance Patterns from Infancy through Early Childhood. In: Food, Culture \& Society 9, 3, S. 287-298.

Mead, Georg H. (1934): Mind, self, and society. Chicago: University of Chicago Press.

Meier-Ploeger, Angelika (2005): Sinnesschulung bei Kindern. In: Engelhardt von, D./Wild, R. (Hrsg.): Geschmackskulturen. Vom Dialog der Sinne beim Essen und Trinken. Frankfurt a. M./New York: Campus, S. 248-261.

Methfessel, Barbara (2014): Essen geben und Essen lehren: Von der Ernährungserziehung zur Esskultur-Bildung. In: Althans, B./Schmidt, F./Wulf, C. (Hrsg.): Nahrung als Bildung. Interdisziplinäre Perspektiven auf einen anthropologischen Zusammenhang. Weinheim u. a.: Beltz Juventa, S. 190-204.

Morris, Ceri (2017): Making sense of education: sensory ethnography and visual impairment. In: Ethnography and Education 12, 1, S. 1-16 [DOI: 10.1080/17457823.2015.1130639], Zugriff am 23.12.16.

Neumann, Sascha (2012): Some children are more different than others: Language practices in Luxembourgian nurseries. In: Qualitative Research Journal 12, 2, S. 183-192.

Neumann, Sascha (2013): Die anderen Dinge der Pädagogik. Zum Umgang mit alltäglichen Gegenständen in Kinderkrippen. In: Nohl, A.-M./Wulf, Ch. (Hrsg.): Mensch und Ding. Die Materialität pädagogischer Prozesse. ZFE Sonderheft, 25. Wiesbaden: Springer, S.107-121.

Orrell-Valente, Joan K./Hill, Laura G./Brechwald, Whitney A./Dodge, Kenneth A./Pettit, Gregory S./Bates, John E. (2007): „Just three more bites”: An observational analysis of parents' socialization of children's eating at mealtime. In: Appetite 48, 1, S. 37-45.

Panagiotopoulou, Argyro/Krompàk, Edina (2014): Ritualisierte Mehrsprachigkeit und Umgang mit Schweizerdeutsch in vorschulischen Bildungseinrichtungen. Erste Ergebnisse einer ethnographischen Feldstudie in der Schweiz. In: Knobloch, P. D. Th./Müller, A./Rühle, S. (Hrsg.): Mehrsprachigkeit - Diversität - Internationalität. Erziehungswissenschaft im transnationalen Bildungsraum. Münster: Waxmann, S. 51-70.

Panagiotopoulou, Argyro/Kassis, Maria (2015): Frühkindliche Sprachförderung oder Forderung nach Sprachentrennung? Ergebnisse einer ethnographischen Feldstudie in der deutschsprachigen Schweiz. In: Geier, Th./Zaborowski, K. U.(Hrsg.): Migration: Auflösungen und Grenzziehungen. Perspektiven einer erziehungswissenschaftlichen Migrationsforschung. ZSB-Reihe, 15. Wiesbaden: Verlag für Sozialwissenschaften, S. 153-166.

Punch, Samantha/McIntosh, Ian/Emond, Ruth (Hrsg.) (2011): Children's Food Practices in Families and Institutions. New York: Routledge.

Punch, Samantha/McIntosh, Ian (2014): „Food is a funny thing within residential child care“: Intergenerational relationships and food practices in residential care. In: Childhood 21, 1, S. $72-86$.

Rabenstein, Kerstin/Steinwand, Julia (2012): Ganztagslehrer/innen in einer familiarisierten Schule: Aufgaben und Zuständigkeiten angesichts multiprofessioneller Kooperation. In: Nerowski, C./Hascher, T./Lunkenbein, M./Sauer, D. (Hrsg.): Professionalität im Umgang mit Spannungsfeldern. Bad Heilbrunn: Klinkhardt, S. 59-72.

Röhl Tobias (2013): Dinge des Wissens. Schulunterricht als sozio-materielle Praxis. Stuttgart: Lucius \& Lucius.

Röhl, Tobias (2015): Die Objektivierung der Dinge. Wissenspraktiken im mathematischnaturwissenschaftlichen Schulunterricht. In: Zeitschrift für Soziologie 44, 3, S. 162-179.

Rose, Lotte (2010): Hauptsache gesund. Zur Medikalisierung des Essens in pädagogischen Einrichtungen. In: Sozial Extra 3/4, 34, S. 50-53.

Rose, Lotte/Sturzenhecker, Benedikt (Hrsg.) (2009): „Erst kommt das Fressen?!“ Über Essen und Kochen in der Sozialen Arbeit. Wiesbaden: Springer.

Rozin, Paul/Fallon, April E. (1987): A perspective on disgust. In: Psychological Review 94, 1, S. 23-41. 
Schulz, Marc (2010a): Bildung während des Essens? Mahlzeiten in Kindertagesstätten im Spannungsverhältnis von Gesundheitserziehung und Bildungsförderung. In: Sozial extra 3/4, 34, S. 38-41.

Schulz, Marc (2010b): Gefrorene Momente des Geschehens. Feldvignetten aus der Kinder- und Jugendarbeit. In: Heinzel, F./Thole, W./Cloos, P./Köngeter, S. (Hrsg.): „Auf unsicherem Terrain“. Ethnographische Forschung im Kontext des Bildungs- und Sozialwesens. Wiesbaden: Verlag für Sozialwissenschaften, S. 171-179.

Schütz, Anna (2015): Schulkultur und Tischgemeinschaft. Eine Studie zur sozialen Situation des Mittagessens an Ganztagsschulen. Wiesbaden: Springer.

Seichter, Sabine (2012): Erziehung und Ernährung. Ein anderer Blick auf Kindheit. Weinheim u. a.: Beltz Juventa.

Schwartz Camille/Scholtens, Petra/Lalanne, Amandine/Weenen, Hugo/Nicklaus, Sophie (2011): Development of healthy eating habits early in life. Review of recent evidence and selected guidelines. In: Appetite 57, 3, S. 796-807.

Strauss, Anselm/Corbin, Juliet (1996): Grounded Theory: Grundlagen Qualitativer Sozialforschung. Weinheim: Psychologie Verlags Union.

Täubig, Vicki (Hrsg.) (2016): Essen im Erziehungs- und Bildungsalltag. Weinheim u. a.: Beltz Juventa.

Wiesemann, Jutta/Lange, Jochen (2015): „Education in a Box“. Die Herstellung schulischer Artefakte in der Lehr- Lernmittelindustrie. In: Zeitschrift für interpretative Schul- und Unterrichtsforschung (ZISU) 4, 4, S. 80-91.

Zischka, Ulrike/Ottomeyer, Hans/Bäumler, Susanne (Hrsg.) (1993): Die anständige Lust. Von Esskultur und Tafelsitten. München: Edition Spangenberg. 


\title{
Der bewegte Körper im Unterricht: zwischen medialer Inszenierung und Manifestation des Leiblichen
}

\begin{abstract}
Zusammenfassung
Schulische Akteure - Lehrpersonen wie Schülerinnen und Schüler - verkörpern im alltäglichen Vollzug des Unterrichts eine organisationsspezifische Ordnung, welche aus institutionalisierten Normen, Regeln und Anforderungen besteht. Sie handhaben ihren Körper dabei medial im Hinblick auf die Ordnung, die sie aufführen; dabei verleiben sie sich diese Ordnung aber auch ein. Im und am Leib wird die institutionell gerahmte Ordnung sowohl als ermöglichende wie auch einschränkende und deformierende Kraft erfahrbar.

Im Artikel wird das Spannungsverhältnis zwischen medialem Körper und phänomenaler Leiblichkeit im Kontext des Unterrichts empirisch untersucht. In Interviews mit Unterstufenlehrpersonen, welche nach der Methode der „Video Elicitation“ durchgeführt wurden, wurde deren Wahrnehmung der körpergebundenen Praktiken im eigenen Unterricht besprochen. Dabei stand die Frage im Zentrum, inwiefern der Körper der im Unterricht Agierenden in seiner Medialität, inwiefern er in seiner Leiblichkeit wahrgenommen wird und in welchem Verhältnis diese Wahrnehmung zur Reproduktion der organisationsspezifischen schulischen Ordnung steht. Als Ergebnis konnten zwei Typen von Wahrnehmungsperspektiven herausgearbeitet werden: Während die eine den mediatisierten und instrumentalisierten Körper in den Dienst der Reproduktion der Ordnung stellt, fokussiert die andere auf den Leib und entdeckt dabei die feldimmanenten Widersprüche und Zumutungen, welche die tagtägliche Aufführung dieser Ordnung für die darin involvierten Subjekte bedeuten.
\end{abstract}

Schlagwörter: Körper, Leiblichkeit, Medialität, Unterrichtswahrnehmung, Lehrperson

To observe and to be observed: How teachers perceive videography and experience its camera gaze in further education

This article analysis how teachers experience to be observed by videography during their teaching. Although video cameras are ubiquitously used in educational research and teacher education and although the possibilities and limitations of video research are reflected mainly in qualitative studies, there is still a dearth of analysis that query the experiences of the observed teachers themselves. This analysis is based on the example of a teacher training course with the aim of professional development, during which lessons were researched by videography. Based on the method of video elicitation, these videos served as stimulus for a reflection in qualitative interviews. The method not only reactivates decision making and thoughts during the teaching, but moreover elicits expectations and attitudes related to the videography itself. The analysis reveals that videography, teaching and teacher habitus produce a complex techno-social assemblage.

Keywords: Videography, Video Elicitation, Teacher, Further Education, Habitus 


\section{Einleitung}

In ihrem Aufsatz „Was verkörpert der Körper des Schauspielers?“ fragt sich Elisabeth Fischer-Lichte (Fischer-Lichte 2004), ob der menschliche Körper überhaupt als Medium begriffen werden könne. Sie untersucht dies anhand der Situation des Performativen schlechthin: der Situation des Schauspielers auf der Bühne. Ihre Antwort: Nein, der menschliche Körper ist nicht als Medium zu denken.

Analytisch entwickelt sie diese Antwort anhand verschiedener theatergeschichtlicher Positionen, welche bis ins 18. Jahrhundert zurückreichen. Grundlagentheoretisch orientiert sie sich an der phänomenologischen Unterscheidung zwischen Körper haben und Leib sein (Plessner 1928). Während Ersteres Voraussetzung dafür ist, dass wir unseren Körper bearbeiten, objektivieren, gezielt einsetzen und gewissermaßen instrumentalisieren können, ist Letzteres eine unhintergehbare Gegebenheit menschlichen Inder-Welt-Seins. Der Leib ist dabei immer der gefühlte, empfundene Körper, welcher in seiner Materialität eigengesetzlich auf unser Fühlen, Denken und Handeln einwirkt. Die Grundlage dafür, dass der Körper überhaupt zum Objekt und zur „Quelle von Symbolbildungen“ (Csordas 1994) werden kann, ist der Leib und unser leibliches In-der-WeltSein.

Im Körper des Schauspielers nun wird diese „Doppelung“ (Fischer-Lichte 2004: 142) von Leib-Sein und Körper-Haben unmittelbar sicht- und erfahrbar. Die Spannung zwischen dem phänomenalen Leib des Schauspielers und der Figur, die er darstellt, verbürgt die Einzigartigkeit der Darstellung. Die Tatsache, dass die Figur von einem ganz bestimmten Körper aufgeführt wird, bewirkt, dass nicht einfach literarisch festgeschriebene Bedeutungen perpetuiert werden, sondern dass in und durch die Aufführung neue Bedeutung erzeugt wird. Der Körper des Schauspielers verkörpert also nicht einfach einen literarischen Text, wie dies die Verkörperungstheorien des 18.Jahrhunderts wollten, sondern er fungiert als „Wirkpotential“ (Fischer-Lichte 2004: 148) für das Entstehen neuer, über den Text hinausgehender Bedeutungen. Für den Zuschauer wiederum wird die Doppelung von phänomenaler Leiblichkeit des Schauspielers und verkörperter Figur als Spannungsverhältnis zwischen Performer und Figur wahrnehmbar. Dabei springt die Wahrnehmung immer wieder von der Wahrnehmung des leiblichen Körpers des Schauspielers zu derjenigen der dargestellten Figur um und umgekehrt.

Die Beantwortung der Titelfrage, ob der Körper des Schauspielers als Medium begriffen werden könne, ist abhängig davon, wie dieses Spannungsverhältnis wahrgenommen wird. Soll im Körper des Schauspielers ausschließlich die Figur respektive der Text sicht- und erfahrbar werden, der Körper also in der Verkörperung aufgehen, so kann er als Medium begriffen werden. In dem Maße, wie jedoch der Leib in Erscheinung tritt, wird die Medialität des Schauspielerkörpers problematisiert.

Dieses Spannungsverhältnis, welches Fischer-Lichte am Beispiel des Schauspielerkörpers herausarbeitet, scheint uns für einen neuen Blick auf den Unterricht vielversprechend. Dabei geht es nicht darum, von vornherein zu unterstellen, dass das Unterrichtsgeschehen als analog zu demjenigen des Theaters zu begreifen sei. Vielmehr soll Fischer-Lichtes theoretischer Ansatz als heuristisches Instrument eingesetzt werden. Das Spannungsverhältnis zwischen leibgebundenem Performer und verkörperter Figur 
soll auf die „Unterrichtsaufführungen“ übertragen werden, um diese versuchsweise in einem neuen Licht erscheinen zu lassen und Forschungsansätze, welche sich mit der Rolle des Körpers im Unterricht beschäftigen, weiterzuentwickeln.

Forschungsarbeiten, wie diejenigen von Alkemeyer und Pille (Alkemeyer 2009; Alkemeyer \& Pille 2008; Pille 2009), untersuchen aus einer praxistheoretischen Perspektive den Erwerb eines professionellen Habitus bei Lehrpersonen und zeigen auf, wie dieser sich als Resultat eines körperlichen Trainings konsolidiert, indem feldspezifische Haltungen, Gesten und Bewegungen eingeübt und an die organisationsspezifischen Anforderungen angepasst werden. Andere Ansätze, wie derjenige von Langer und Richter (Langer 2008, 2011; Langer \& Richter 2015), legen einen diskursanalytischen Fokus auf die verschiedenen körpergebundenen Disziplinierungspraktiken, wie sie in einschlägiger, praxisnaher Literatur einerseits diskursiv gefordert, andererseits von Lehrpersonen praktisch umgesetzt und gedeutet werden. Beiden Forschungsrichtungen ist gemeinsam, dass sie sich auf die instrumentelle Zurichtung des Lehrerkörpers im Hinblick auf dessen Einsatz als Medium der Steuerung von Unterrichtsprozessen konzentrieren. Die Spannung zwischen der Leiblichkeit als wahrgenommener, erlebter Erfahrung und dem Körper als medial gehandhabtem Instrument der Inszenierung wird dabei höchstens in Randbemerkungen oder Fußnoten angesprochen (Alkemeyer \& Pille 2008: 141; Langer 2008: 275).

Um diese soll es jedoch im Folgenden gehen. Die übergreifende Zielfrage, der wir unsere Überlegungen unterordnen, lautet, inwiefern der Körper der im Unterricht Agierenden in seiner Medialität, inwiefern er in seiner Leiblichkeit in Erscheinung tritt und in welchem Verhältnis die beiden Modalitäten zueinander stehen.

Wir werden hierzu empirisches Material aus einem laufenden Forschungsprojekt hinzuziehen. Vorbereitend werden wir in einem ersten Schritt das Verhältnis von Performativität, Medialität und Körperlichkeit im Hinblick und mit dem Fokus auf unser Material näher zu bestimmen versuchen. Anschließend werden wir Forschungskontext und Methode kurz erläutern. Dann werden wir das Material - es handelt sich um zwei Fallbeispiele - vorstellen und analysieren. Schließlich werden wir versuchen, auf unsere Hauptfrage zurückzukommen und unsere Ergebnisse mit den theoretischen Anfangsüberlegungen zu verklammern.

\section{Performativität, Medialität und die Rolle des Körpers im Unterricht}

Dass Performativität und Medialität verwandte Konzepte sind, scheint - wenn man an deren konstruktiven Grundgestus denkt - naheliegend. Krämer (2004: 23) ortet eine gewisse „Gleichgerichtetheit von Positionen der Performativitätsdebatte und dem Mediengenerativismus“, welche sich in der „Privilegierung des Machens im Darstellen, des Erzeugens im Widerfahren“ zeige. Diese Ähnlichkeit im Gestus der mit den beiden Begriffen verbundenen Theorietraditionen (vgl. einführend Hempfer \& Volbers 2011; Kloock \& Spahr 2012) sollte einen aber nicht davon entbinden, die jeweils unterschiedlichen Akzentuierungen herauszuarbeiten. So steht im Falle der Medialität eines Phä- 
nomens oder Vorgangs primär das Mittel der Übertragung und damit die Materialität im Vordergrund, während bei der Performativität das Ereignis, die Aufführung oder der Vollzug ins Auge gefasst werden. Während Ersteres durchaus auch zeit- und situationsunabhängig gedacht und untersucht werden kann - man denke nur an die Analyse der Funktionsweise technischer Medien -, so ist Letzteres nur als Geschehen in actu, eben im Vollzug untersuchbar. Denkt man Performativität und Medialität zusammen, so überblenden sich Aufführungscharakter und Materialität. Das, was ein Akteur in einem Vermittlungsgeschehen performativ hervorbringt, wird von Betrachtern auf eine Weise rezipiert, welche die Intention des Vollzugs überschreitet; die Qualität dieser Überschreitung liegt auch in der Materialität des Mediums begründet, sie ist immer auch die „Spur“ des Mediums (Krämer 1998).

Überträgt man diese Überlegungen nun auf das Feld des schulischen Unterrichts und berücksichtigt man überdies die Ausführungen, die wir zu Beginn im Anschluss an Fischer-Lichte formuliert haben, so nimmt das Phänomen „Unterricht“ ganz bestimmte Konturen an. Grundsätzlich orientieren wir uns in der theoretischen Konzeption von Unterricht an aktuellen ethnographischen, praxistheoretischen und interaktionistischen Unterrichtstheorien, welche sich bemühen, die normativen Rahmungen, welche die Unterrichtsforschung begleiten, auszuklammern und die Sozialität und die Kontingenz des Unterrichts zu betonen (Breidenstein 2010, Meseth u. a. 2012). Wir integrieren in diese theoretischen Ansätze nun Dimensionen, die wir oben im Zusammenhang mit Performativitäts- und Medialitätstheorien herausgearbeitet haben. Damit fokussieren wir ein Verständnis von Unterricht, welches - auf ein paar Stichworte verkürzt - den praktischen Vollzug und das körperliche Aufführen (Göhlich 2001), die Zeitgebundenheit und den Bezug zur Gegenwart (Dirksmeier \& Helbrecht 2008) und das Wechselspiel von Planung und Emergenz (Fischer-Lichte 2012) in den Vordergrund rücken.

Unterricht ist in dieser Perspektive also ein Geschehen, bei dem Tag für Tag Lehren und Lernen aufgeführt werden. Diesen Aufführungen liegt ein jeweiliger „Text“ oder eine „Konvention“ (Göhlich \& Zirfas 2001: 32) zugrunde: ein disziplinär organisierter Wissenskanon z. B., oder soziale Normen und Haltungen, kulturelle Übereinkünfte, institutionell bedingte Regeln, Peer-Rituale etc. Im täglichen Vollzug des Unterrichts wird dieser „Text“ wiederholt und eingeübt. Im Einüben wird nicht nur der „Text“ aktualisiert; vielmehr üben die Akteure damit auch eine bestimmte Denk- und Wahrnehmungsweise ein, entwickeln habitualisierte Haltungen und Verhaltensweisen, welche als Effekte einverleibter institutioneller Strukturen gelten können.

Üben basiert auf Wiederholung. Aber obschon es sich jeweils um den gleichen Text handelt, ist dessen Aufführung nicht nur immer wieder anders, sondern auch anders als gedacht und geplant. Durch die raumzeitliche Verschiebung, welcher die Wiederholung oder das Zitat unterliegt, taucht in jeder Wiederholung etwas Neues auf, wird Raum gegeben für die Emergenz von Unvorhergesehenem.

Die Medien nun, die in diesen Inszenierungen zum Einsatz kommen, sind vielfältig - gemeinhin, d. h. innerhalb eines (fach-)didaktischen Rahmens denkt man an audiovisuelle Geräte, an Lehrmittel und didaktische Objekte. Uns interessieren jedoch im Folgenden der menschliche Körper und die Frage, inwiefern und unter welchen Bedingungen er in Unterrichtsaufführungen als Medium eingesetzt und begriffen werden kann. Der Körper fungiert, wenn medial eingesetzt, als Träger und Vermittler einer sym- 
bolischen Ordnung; im Kontext des Unterrichts wird diese in Form institutionalisierter Normen und Regeln und kulturell geformtem und tradiertem Wissen wirksam. Dem instrumentellen Einsatz des Körpers als Medium steht jedoch der Leib in seiner ungerichteten, phänomenalen Selbstbezüglichkeit gegenüber. Die menschlichen Körper im Unterricht - die Körper der Lehrpersonen wie diejenigen der Lernenden - denken wir also analog zum Körper des Schauspielers auf der Bühne. Wie dieser aktualisieren jene das Spannungsverhältnis zwischen phänomenalem Leib und semiotischem Körper, dem sie unterworfen sind. Aber wenn der Körper des Schauspielers eine literarische Figur verkörpert, so derjenige der schulischen Akteure eine soziale Ordnung. Verkörperung meint dabei einen doppelten Prozess: der Prozess des Aufführens ist auch ein Prozess der Einverleibung. Unterricht ist ein Geschehen, in dem ein soziales Regelsystem wiederholt zitiert und vollzogen wird. Wir denken hierbei an Interaktions- und Kommunikationsregeln, an Rituale und didaktische Routinen, mit deren Hilfe Unterricht immer wieder hergestellt wird (Kalthoff \& Kelle 2000). Vollzugsinstrumente dieses Regelsystems sind nicht nur sprachliche Explikationen, sondern auch körpergebundene Praktiken wie Gesten, Blicke, Haltungen, Bewegungen im Raum etc. Schulische Akteure - dies die Annahme - verkörpern die soziale Ordnung des Unterrichts. Sie handhaben ihren Körper dabei medial und funktional im Hinblick auf die Ordnung, die sie aufführen. Gleichzeitig verleiben sie sich jedoch im Akt der Aufführung diese Ordnung auch ein. Im und am Leibe wird die organisationsspezifische Ordnung erfahrbar, in der individuellen Physis wird sie spürbar - sowohl in ihrer ermöglichenden wie in ihrer einschränkenden und deformierenden Kraft.

Leibliche Manifestationen sind also nicht von präkultureller, naturalisierter Qualität. Der Leib ist in unserem Verständnis kein a-gesellschaftliches Apriori, auf den das Subjekt einen ,Exklusivitätsanspruch“ hätte (vgl. kritisch Hirschauer 2008: 180), sondern vielmehr eine bestimmte Verfassung des Subjektes in seinem Verhältnis zur Welt (Csordas 1994). Der Leib, so formulieren wir im Anschluss an eine „negative Medientheorie“ (Mersch 2006), ist in seiner Materialität und Eigenlogik am ehesten negativ umschreibbar. Er manifestiert sich als Grenze und Widerständigkeit, als Ort, wo die Mediatisierung des Körpers an ihre Grenze stößt und das Vermittlungsgeschehen gestört oder - durchaus auch spielerisch - unterlaufen werden kann.

Ausgehend von dieser theoretischen Rahmung wollen wir im Folgenden die Frage nach dem Verhältnis von medialem Körper und phänomenaler Leiblichkeit im Kontext des Unterrichts empirisch untersuchen. Wir fokussieren dabei auf die Lehrperson. Folgende Fragen sind leitend: Wie nehmen die Lehrpersonen in den alltäglichen Aufführungen von Unterricht das Verhältnis von Leib und Körper wahr? Welche „Texte“ versuchen sie mit ihren Körpern aufzuführen? Welche Regeln und Normen, welche „Konvention“ bemühen sie sich mit ihrem Lehrer-Körper zu mediatisieren? Und welche Rolle spielt in ihrer Wahrnehmung dabei der Leib? 


\section{Körpergebundene Inszenierungen in Unterrichtsprozessen- Fallbeispiele aus einem schweizerischen Lehrerweiterbildungsprojekt}

\subsection{Projektkontext}

Das Forschungsprojekt, anhand dessen wir diese Fragen empirisch untersuchen wollen, soll im Folgenden kurz vorgestellt werden: Es handelt sich um ein Projekt an der PH Bern, welches einen Weiterbildungs- und einen Forschungsteil umfasst. Ziel der halbjährigen Weiterbildung war es, über das Mittel tanzkünstlerischer Arbeit den Lehrpersonen die Gelegenheit zu geben, sich mit den stummen, nonverbalen Praktiken des Unterrichts auseinanderzusetzen. Über die Dekontextualisierung des habituell gewordenen Bewegungsrepertoires und dem experimentellen Spiel mit feldspezifischen Haltungen, Gesten und Blicken sollte die Möglichkeit geboten werden, sich mit eben diesen habituellen Mustern - Bewegungs-, Denk- und Wahrnehmungsschemata - auseinanderzusetzen. Im Zentrum der Begleitforschung stand und steht dagegen die Erforschung der Körperkonzepte der Lehrpersonen; untersucht wird hier die Unterrichtswahrnehmung der Lehrpersonen. Gefragt wird, welche Bedeutung sie den nonverbalen und körpergebundenen Praktiken zumessen. Welche körperlichen Aktionen nehmen sie überhaupt wahr, wie interpretieren sie sie und welche Bedeutung haben sie für ihre Unterrichtspraxis?

Für die Erhebung der Daten in der Begleitforschung wurden die Methoden der Unterrichtsvideographie und der „Video Elicitation“ (Henry \& Fetters 2012) eingesetzt. Der Unterricht der acht Kursteilnehmerinnen - es handelt sich um weibliche Unterstufenlehrkräfte unterschiedlichen Alters - wurde im Verlaufe von ca. sechs Monaten dreimal videographiert, wobei den Lehrpersonen die Wahl der Stunde, des Fokus und die Kameraregie unterlag. Nach dem Durchschauen des gesamten Videos wählten die Teilnehmerinnen jeweils einzelne Sequenzen aus, welche in einem nach den methodischen Regeln der „Video Elicitation“ ausgerichteten Interview besprochen wurden. Ziel dabei war es, über die Besprechung der Sequenzen Assoziationen und Erinnerungen an Entscheidungs- und Denkprozesse hervorzulocken, die während der konkreten Situation stattfanden.

Bei der Auswertung der Daten folgte auf die Transkription, bei welcher gleichzeitig die Übersetzung ins Hochdeutsche vorgenommen wurde, ein offenes und nahe am Text angelegtes Codieren. In einem nächsten Schritt wurde axial codiert, um dann über fallinterne sowie fallvergleichende Analysen Kernkategorien zu bestimmen. Die Fallrekonstruktionen erfolgten entlang dieser Kernkategorien und mündeten in eine Typenbildung. Der Typus erfasst dabei die Wahrnehmungsperspektive der Lehrpersonen, d. h. die Art und Weise, wie die Lehrpersonen die körpergebundenen Praktiken des Lehrens und Lernens wahrnehmen und beurteilen, und die Bedeutung, die sie ihnen im Unterricht zuweisen.

Anhand der zwei folgenden Fallbeispiele sollen zwei diesbezüglich kontrastierende Typen vorgestellt werden. Die Gegenüberstellung zweier unterschiedlicher Wahrnehmungsperspektiven ist also analytisch motiviert und Resultat einer Abstraktion. Die exemplarisch ausgewählten Textpassagen, welche wir zur Illustrierung heranziehen, 
entstammen jeweils aus den ersten zwei von insgesamt drei Interviews, die mit den Teilnehmerinnen durchgeführt wurden.

\subsection{Fallbeispiel 1: Körpergebundene Inszenierungen als Instrument zur Herstellung von Disziplin}

L1 unterrichtet eine jahrgangsübergreifende Klasse der Unterstufe (1./2. Klasse) in einer ländlichen Gemeinde der Schweiz. Die folgenden Interviewauszüge stammen vorwiegend aus dem ersten Interview; Gegenstand dieses Interviews ist die Videographie einer Doppelstunde in Bildnerischem Gestalten. Die Kamera war hinten im Schulzimmer positioniert, damit sie gewissermaßen in die räumliche Struktur des Zimmers integriert blieb und die Lernenden nicht abgelenkt wurden. Der Kamerafokus erfasst sowohl die ganze Klasse wie auch die Lehrkraft.

In der Besprechung der Videographie setzt L1 folgende zwei Foki:

Ich sage dir vielleicht, was mir nachher aufgefallen ist und warum ich es anschauen möchte. /I: $\mathrm{mhm} / /$ Es ist mir aufgefallen, dass ich teilweise (+) also, dass ich recht oft in Bewegung bin. /I: $\mathrm{mhm} / /$ Und dass ich teilweise eine Bewegung beginne und diese nachher abrupt abbreche und etwas/ in eine andere Richtung gehe. [...] Das ist das eine, was ich gedacht habe, ehm: möchte ich für mich selbst mal anschauen. Und das andere ist, ehm: es war mir nicht bewusst, wie sehr/ wieviel ehm: ich gestikuliere mit der Körpersprache. Also den Körper einbeziehe, um Sachen zu verdeutlichen. Jetzt habe ich auch gerade wieder so/ Oder? /I: $\mathrm{mhm} / /$ Und das war mir also wirklich nicht/ Das war mir nicht bewusst. Und mit dem Schauen dieses Videos dachte ich *poah:* [LACHT] Eben diesen Körper, wie die Tanzkursleiterin sagte, der Körper ist sehr präsent im Unterricht und ist ehm: ein wichtiger/ ein wichtiger Botschafter.

L1 konzentriert sich im ersten Interview auf zwei Sequenzen: Die erste Sequenz wählt sie aus, weil sie dort bei sich eine abrupte Bewegung bemerkt hat. L1 hält fest, dass ihr die eigene Bewegtheit im Allgemeinen sowie schnelle Bewegungswechsel aufgefallen sind und sie sich vertieft damit auseinandersetzen möchte. Eine zweite Sequenz interessiert sie, weil in ihr verbale Erklärungen, die sie der Klasse gibt, mit Gesten einhergehen. Durch das Anschauen des Videos ist ihr bewusst geworden, wie sehr sie sich dieser Kommunikationsform bedient. L1 wiederholt in der Folge mehrfach ihr Erstaunen darüber, dass sie auch körpersprachlich agiert, was hier im Ausruf ,ppoah“ zum Ausdruck kommt.

Ausgehend von der aus ihrer Perspektive abrupt abgebrochenen Körperbewegung entfaltet L1 in der Besprechung der ersten Sequenz die Überzeugung, dass ein ruhiger Lehrer-Körper eine positive Wirkung haben kann. Hier, wie auch in späteren Zitaten, verweist L1 auf die Logopädin, die sich phasenweise ebenfalls im Raum befindet und mit der L1 im Teamteaching unterrichtet. Für L1 dient die Logopädin als Kontrastfolie zu sich selbst:

Aber siehst du, sie [gemeint ist die Logopädin] steht viel oft da, so: So wie ein Fels hier, so in der Brandung. So, weißt du, so: [MACHT ES VOR] und schaut. /I: Ja.// Und ich/ ich (+) Ich gehe hier und dort und/ Schon nicht laut - weißt du - absolut nicht laut, aber die Bewegung selbst kann bereits Unruhe bringen. /I: Ja.// Ich habe mich nun fast ein bisschen als unruhig/ Gegenüber der Logopädin habe ich mich als unruhig erlebt. 
Im assoziativen Teil der Sequenzbesprechung verweist L1 auf die Bewegung, die sie gestört hat. Ausgehend von dieser Bewegung thematisiert sie die Norm des „RuhigSeins“, welche sie in der Logopädin verwirklicht sieht. Mit der Metapher des Felsens, der in der Brandung steht, erfährt die Logopädin eine Idealisierung: So ruhig sie dasteht, vermag sie der Brandung bzw. der Klasse oder den Schülerinnen und Schülern standzuhalten. Sich selbst erlebt L1 hingegen als unruhig. Aus ihrer Sicht ist dieses In-Bewegung-Sein problematisch, da es möglicherweise Auswirkungen auf die Situation hat und - nicht erwünschte - Unruhe bringen kann. Es wird deutlich, dass L1 die körperlichen Inszenierungen des Lehrkörpers funktional im Hinblick auf Herstellung bzw. Aufrechterhalten von Ruhe und Disziplin begreift. Das Ideal eines ruhigen Körpers und damit eines ruhigen Unterrichtsklimas kann - so ihre Vorstellung - durch Disziplinierung des eigenen Körpers erreicht werden:

Ich hatte den Eindruck, es würde die Ruhe, die die Kinder ausstrahlen/ (+) Für mich selbst/ Ich hatte diese Ruhe nicht ehm: übernommen. [...] Ich möchte sie eigentlich unterstützen. Wenn sie schon so ruhig sind. Dass wenn sie schon so ruhig sind, dass ich mich eben auch ruhig halte. /I: Ja.// Und ich kann mich erinnern, eine Klasse gehabt zu haben, die es nicht ertragen hat, wenn man viel herumgegangen ist. Dann wurden sie gerade unruhig. /I: $\mathrm{mhm} / /$ Und da hatte ich wirklich in der Planung/ Das kommt mir jetzt gerade in den Sinn. Das ist mir vorher/ Das hatte ich ganz vergessen gehabt. Und dort hatte ich auch in der Planung überlegen müssen: Wann bin ich da vorne? Wann halte ich mich da vorne still? /l: $\mathrm{mhm} / /$ Wie organisiere ich es, wenn ich schauen will, wo sie sind. Eben vielleicht auch mal wie die Logopädin, so [MACHT ES VOR.]. /I: mhm// Das muss ich noch etwas schauen.

L1 erfährt die Lernenden hier als Ruhe ausstrahlend. Sie selbst vermag aber diese Ruhe nicht zu übernehmen. Dies möchte sie ändern, indem sie sich selber zur Ruhe zwingt. Das Aufrechterhalten von Ruhe erfolgt damit über einen strategischen Einsatz des eigenen Körpers. Dies wird deutlich, als sie von der Reaktion einer Klasse erzählt, die ausgesprochen sensibel auf das Herumgehen der Lehrperson reagierte. Ausgehend von der Erfahrung mit dieser Klasse thematisiert sie den strategischen Einsatz des Lehrerkörpers, der auf bewusster Planung basiert: Wo soll er stehen? Wann soll er sich stillhalten? Wie soll der Blick geführt werden und inwiefern soll er sich am Vorbild „Fels in der Brandung" orientieren?

Das Herstellen von Ruhe soll folglich nicht über äußeren Zwang oder aktive Disziplinierung (z.B. mündlicher Ermahnung) erfolgen, vielmehr verfolgt L1 das Ideal, Disziplin im Klassenraum über Selbstdisziplin herzustellen, über einen planvollen und bewusst gestalteten Einsatz des eigenen Körpers. Die Disziplinierung des eigenen Lehrerinnenkörpers geschieht dabei über dessen Mediatisierung: gezielte Bewegungsabläufe, kontrollierte Blickarbeit, strategische Verortung im Raum - der Körper dient als Medium zur Herstellung von Ruhe. Damit dies gelingt, müssen unmittelbare, chaotische und ungerichtete Bewegungsimpulse neutralisiert werden. Die ,abrupten“ Bewegungen, die L1 beim Betrachten der Videographie sofort negativ aufgefallen sind, sind ein Irritations- und Störfaktor, den sie als Anlass nimmt, um gezielt an ihrem Körper zu arbeiten - wie sie an anderer Stelle sich vornimmt.

In der zweiten Sequenz, welche L1 auswählt, denkt sie am mediatisierten Einsatz des Körpers weiter und führt das Thema „Körpersprache“ ein; Mimik und vor allem 
Gestik können in der Wahrnehmung von L1 die gesprochene Sprache unterstützen oder gar ersetzen:

Und (+) also ehm: man könnte mal versuchen, (+) einmal nur mit Körpersprache gewisse Sachen/ einen Auftrag zu geben. /I: $\mathrm{mhm} / /$ Die Worte mal wegzulassen. [...] Ja, genau. (+) Das habe ich mir überlegt. Also Körpersprache finde ich eben nicht schlecht als Unterstützung.

Gezielt eingesetzte Körpersprache scheint L1 also vielversprechend, da daraus mögliche Unterstützung resultiert. Dies zeigt sich in der Beschreibung der gleichen Unterrichtssequenz an anderer Stelle im Interview, wo L1 festhält, dass sie alle ihre Anordnungen mit Bewegung, insbesondere mit den Händen unterstütze und Kinder bisweilen lediglich über Mimik und Gestik maßregle bzw. zum Zuhören animiere. Sie bedient sich damit gewissermaßen einer anderen Sprache, nämlich der Körpersprache, um die sie die gesprochene Sprache ergänzen oder sogar ersetzen möchte.

Neben dem Ideal des ruhigen Körpers, der Disziplin vermitteln kann (Sequenz 1), erscheint also in dieser Sequenz ein bewegter Körper, der aber in Form von ritualisierten Gesten letztlich auch als Instrument eingesetzt wird, um einen störungsfreien Unterricht zu garantieren. Dies spiegelt sich in einer anderen Sequenz aus dem zweiten Interview wider, in dem L1 überprüfen möchte, ob die „Arbeit“ an ihrem Körper, die sie sich vorgenommen hat, erfolgreich gewesen ist. Sie möchte hier schauen, ob sie die Ziele, die sie sich gesetzt hat - „dass ich mich still halte und sitze“ sowie „mehr einfach mit Gesten [zu] arbeiten“ - umsetzen kann:

Ehm: diese Sequenz hat mir von daher sehr gut gefallen. [...] Und nun schaue ich, damit ich mög-

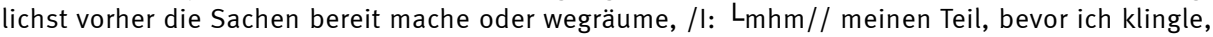
damit ich wirklich dastehen kann und Ruhe/ (+) Ruhe geben kann. (++) Oder eben auch damit/ Das, was ich zum Ziel hatte. Ich habe hier nonverbal (+) viele Dinge gemacht. (++) Die sich nun einfach ein wenig eingeschliffen haben. /l: $\mathrm{mhm} / /(+)[. .$.$] Aber für solche Sachen, für irgendwas, was wir$ abgemacht haben oder ich von ihnen verlange, dass ich dies eben nur durch ein Zeichen bestätige oder nochmals zeige, (+) eben ohne Worte.

L1 evaluiert die Sequenz positiv, weil es ihr jetzt gelingt, sich so zu organisieren, dass Ruhe gewährleistet ist. Dabei stellt sie klar, dass sich die nonverbalen Inszenierungselemente bewähren, insbesondere dann, wenn sie „eingeschliffen“, d. h. ritualisiert sind. Sie könnten die mündliche Sprache - als Quelle von Tönen, Geräuschen und somit potentieller Unruhe - ersetzen. Nonverbale Zeichensprache vermittelt Inhalte und Haltungen stumm; ritualisierte Gesten sollen wie Signale funktionieren - eindeutig und informationstheoretisch gewendet ohne Rauschen. Sind die Lernenden entsprechend konditioniert, so werden die gesendeten Signale eindeutig verstanden und führen scheinbar unmittelbar zu den adäquaten Handlungen. Die Stimme als mögliche Geräuschquelle bzw. als Störfaktor ist eliminiert. Die Lernenden sollen wissen, was zu tun ist bzw. wie sie sich verhalten sollen und erhalten diese Informationen lautlos übermittelt.

Zusammenfassend lässt sich festhalten, dass für L1 ein ruhiges Arbeitsklima und ein reibungsloser Unterrichtsverlauf zentrale Desiderata ihrer Unterrichtspraxis darstellen. Dies soll über die Mediatisierung des eigenen Lehrerinnenkörpers erreicht werden: das Ruhig-Halten des eigenen Körpers, der strategische Einsatz von Bewegung und das Benutzen von gezielten Gesten als Ersatz für verbalsprachliche Anweisungen dienen 
dabei der Herstellung einer normativen Ordnung, die als solche nie in Frage gestellt wird. Dort, wo die Mediatisierung nicht restlos gelingt und ihr der Leib gewissermaßen dazwischen funkt - in den unwillkürlichen Bewegungsimpulsen, dem ruhelosen Umhergehen -, hakt L1 in der „Video Elicitation“ ein und interpretiert diesen Mangel als persönliches Scheitern. Strukturell bedingte widersprüchliche Anforderungen an den Lehrerinnenkörper werden dabei nicht reflektiert, vielmehr werden mögliche körperliche Optimierungen angestrebt, um auf diesem Wege die Unterrichtsordnung (wieder-) herzustellen oder aufrechtzuerhalten. Der Lehrerinnenkörper wird aus der Perspektive von L1 als Medium wahrgenommen, das zielgerichtet eingesetzt werden kann. Der Körper fungiert, wie L1 im eingangs zitierten Interviewausschnitt festhält, als „Botschafter"; in den Unterrichtsinszenierungen von L1 dominiert die Idee des Körper-Habens, wohingegen der Leib eine neutralisierende Bearbeitung erfährt.

\subsection{Fallbeispiel 2: Manifestationen des Leibes als Reflexionsanlass}

Eine andere Wahrnehmung körpergebundener Unterrichtsphänomene findet sich bei L2 . L2 unterrichtet eine erste Klasse in einer Agglomerationsgemeinde einer größeren Stadt in der Schweiz. Die folgenden Interviewauszüge stammen mehrheitlich aus der ersten Interviewphase, in der L2 eine Lektion im Fach NMM (Natur Mensch Mitwelt) videographieren ließ. Die Kamera fokussierte den Sitzkreis, in dem das Unterrichtsgeschehen stattfand und wo sich auch die Lehrperson befand. Im Interview wählt L2 drei Sequenzen aus; dabei interessiert sie sich vor allem dafür, was beim Erzählen im Kreis oder bei örtlichen Wechseln der Kinder geschieht und welche Interaktionen oder Unruhemomente in welcher Form und aus welchem Grund erfolgen. Obwohl L2 von der Kamera miterfasst wurde, liegen ihr Wahrnehmungsfokus und ihr Erkenntnisinteresse auf den Kindern. In ihrer „Video Elicitation“ stehen nicht die körpergebundenen Inszenierungen des Lehrerinnenkörpers im Zentrum, sondern die Frage, was sich im körperlichen Verhalten der Schüler und Schülerinnen manifestiert.

Die folgenden Interviewausschnitte stammen aus der Besprechung einer Videosequenz, welche zeigt, wie die Kinder über längere Zeit im Kreis sitzen und ihnen von L2 eine Geschichte vorgelesen wird:

Ja, man sieht viele Kinder, bei denen ich den Eindruck habe, sie mögen ${ }^{1}$ nicht mehr, die sind müde. Schüler4 liegt auf der Bank, ehm: Schülerin4 spielt mit den Haaren des Kindes vor ihr, Schüler1 und Schülerin1 turnen auch wieder irgendetwas zusammen herum, Schüler5 ist sich auch am auf und ab räkeln und ihm ist, glaube ich, auch nicht mehr so wohl. Schüler6 sieht man sich auch zwischen durch einmal strecken und eben gähnen. Also die Kinder zeigen eigentlich sehr stark, dass sie müde sind, also nicht müde, weil es schon am Abend wäre, sondern weil sie einfach in dieser, ja, Situation (+) jetzt einfach etwas anderes bräuchten. [...] Da sieht man die Differenz zwischen meiner Vorstellung, die ich habe, wie es jetzt, was ich machen möchte, und wie die Kinder darauf reagieren, was für sie eigentlich gerade das richtige wäre. (+) Und dann beginnen sie halt, sich selbst zu bewegen oder zu beschäftigen [BEIDE LACHEN] oder abzulenken. Ja.

In dieser Interviewpassage beschreibt L2 relativ detailliert die Körper der Schüler und Schülerinnen. Sie interpretiert deren körperliche Haltungen und Aktivitäten als Aus-

1 Mundartlicher Ausdruck; dem mundartlichen Ausdruck „nicht mehr mögen“ entspricht in der Hochsprache am ehesten „ermüdet sein“, „keine Lust mehr haben“ 
druck von Müdigkeit bzw. als Ausdruck des Wunsches nach einer anderen Beschäftigungs- oder Unterrichtsform. Sie stellt dann fest, dass sie sich während der Stunde nicht von ihrem Plan bzw. ihrer Planung zu lösen vermochte. Erst durch das Sehen des Videos nimmt sie die körperlichen Manifestationen der Lernenden wahr und schließt daraus, dass eine andere Unterrichtsform adäquater gewesen wäre.

Bereits hier wird eine im Vergleich zu L1 differente Wahrnehmung des Körpers im Unterrichtsgeschehen erkennbar: Im Vergleich zu L1, die den Lehrerinnenkörper in seiner Medialität wahrnimmt, erfährt L2 die bewegten Körper der Lernenden als Ausdruck von leiblicher Verfasstheit, von der aus sie ihre Unterrichtsplanung reflektiert. Der bewegte Körper der Schülerinnen und Schüler erscheint nicht als Anlass zu Disziplinierung, sondern als Ausdruck der Überforderung mit der Situation.

Dies zeigt sich an einer anderen Stelle, wo zwei Schüler miteinander interagieren und mit Handzeichen kommunizieren. Wiederum von einer relativ genauen Beschreibung der körperlichen Aktivitäten der Lernenden ausgehend kommt L2 zum Schluss, dass die Schüler nicht mehr sitzen und zuhören und dies auch zum Ausdruck bringen wollten. Von der Beobachtung der (Kinder-)Körper ausgehend hält L2 fest, dass das Unterrichtsarrangement für die beiden Schüler nicht mehr passe. Sie führt weiter aus:

[...] Wie soll ich sagen? - (+) Ich kann sie gut verstehen. Und alles, was drum herum arrangiert ist, (+) das können sie ja nicht ändern, es ist nicht/ (+) Also ja, in diesem Moment sage ich ja ,Schülerin1 und Schüler1 hört auf' so quasi, und ,Haltet euch jetzt noch still', und ,Wir sind jetzt noch da.' Aber eigentlich (+) ja, ist es gewissermassen Unrecht getan, wenn man so die äußeren Faktoren anschaut, die zusammen spielen. $(00: 12: 11)$

L2 zeigt Verständnis für die Lernenden, die das Unterrichtssetting nicht mitbestimmen können. Sie reflektiert dabei ihre Einforderung von Ruhe und kommt zum Fazit, dass sich diese nicht rechtfertigen lässt. Blieb im tatsächlichen Vollzug des Unterrichts die Norm des Still-sein-Müssens in Kraft, erachtet L2 in der Reflexion dieser Unterrichtssequenz das Durchsetzen der Norm als ,unrecht“.

In der Folge fokussiert L2 den Moment, in dem die Geschichte zu Ende erzählt ist und sie die Kinder zum Aufstehen auffordert, da danach ein Lied gesungen wird. Im assoziativen Teil der Sequenzbesprechung hält L2 fest, dass dieser Wechsel für die Kinder gewissermaßen erlösend gewesen sei und im Anschluss daran „recht $(+)$ recht viel untereinander" geschehe. Auch diese Sequenz beschreibt L2 detailliert und reflektiert daraufhin das Unterrichtsgeschehen:

Ich finde, es gibt für die anderen Kinder rundherum halt einen Moment, in dem man warten muss bis dann jedes steht und bereit ist. (+) Ehm: (+) Ja. Eben eigentlich finde ich, Kinder dürfen zeigen was/

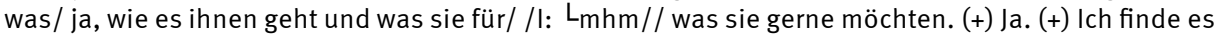
noch schwierig. Es ist eine Gratwanderung zwischen wie viel will ich, dass die gesamte Klasse dies zusammen macht oder ich weiß auch nicht. Wenn er hätte/ Wenn ich gesagt hätte: ,Wenn du schnell liegen willst, dann geh doch ein bisschen auf die Matratze und / Ja. Würde von mir her auch drin liegen. /I: mhm// Aber dann würden wohl alle auf die Matratze wollen. [BEIDE LACHEN] Ja. Eigentlich erwarte ich hier sehr viel, finde ich.

Für L2 steht der Aspekt, dass alle warten müssen, dem Anspruch gegenüber, dass der Schüler zum Ausdruck bringen darf, wie es ihm geht und was er möchte. Sie erfährt die- 
sen Widerspruch (viele Kinder in einer Klasse, die funktionieren sollen vs. Bedürfnisse einzelner Kinder) als „schwierig“ bzw. als Grat, auf dem Lehrpersonen gehen müssen. Grundsätzlich erachtet sie es als legitim, dass der Schüler sich auf einer Matratze hinlegen kann, wenn er müde ist, sieht sich dann aber mit dem Problem eines möglichen «Dammbruchs» konfrontiert: Was wäre, wenn dann alle auf die Matratze möchten? Damit gelangt L2 über die Betrachtung des Schülers und dem Analysieren seiner Bedürfnisse zur Frage nach strukturellen Bedingungen, in denen sie sich als Lehrkraft zu bewegen hat.

Zusammenfassend kann festgehalten werden, dass L2 über die Beobachtung körperlicher Manifestationen der Kinder in die Reflexion der Unterrichtsordnung geht. So hinterfragt sie ihre Unterrichtsgestaltung (Rhythmisierung) und stellt dabei auch die Norm des Ruhig-Seins in Frage. Die bewegten Körper erfahren nicht in erster Linie eine Problematisierung, sondern werden als Ausdruck (leiblicher) Verfasstheit verstanden, auf deren Basis weiterführende Überlegungen erfolgen. Was L2 verändern möchte, sind nicht die bewegten Körper der Lernenden. Diese müssen folglich nicht in erster Linie diszipliniert werden. Vielmehr sind äußere Faktoren derart zu gestalten, dass sich die Lernenden in einem ihnen adäquaten Lernarrangement befinden.

L2 hält fest, dass sie auf die (leibliche) Verfasstheit der Schüler und Schülerinnen eingehen und ihnen die Möglichkeit geben möchte, sich nach den eigenen Bedürfnissen zu richten. Gleichzeitig sieht sie sich mit der Problematik konfrontiert, dass dann vielleicht alle dasselbe haben möchten und dies nicht für alle gleichzeitig realisierbar wäre. Sie erfährt den Widerspruch zwischen dem Anspruch, eine Klasse als Ganze zu führen und dem Prinzip der Gleichheit aller Genüge zu tun und dem Wunsch, die Individualität einzelner Lernender zu berücksichtigen, als schwierig. Damit erfasst sie ein strukturelles Schulproblem.

\section{Der Körper im Unterricht: zwischen Medialität und Leiblichkeit}

Anhand zweier Fallbeispiele wurden zwei unterschiedliche Perspektiven auf körpergebundenes Geschehen im Unterricht dargestellt. Aus analytischem Interesse wurden die beiden Wahrnehmungsmodi kontrastiv ausdifferenziert.

Im Fallbeispiel 1 richtet sich der Wahrnehmungsfokus der Lehrperson auf den Lehrkörper und seine Inszenierungspraxis. In der „Video Elicitation“ wurde deutlich, dass L1 ihren Körper medial einsetzt: durch die Selbstdisziplinierung des Lehrerinnenkörpers versucht sie, die als notwendig erachtete Disziplin im Schulzimmer zu schaffen und aufrecht zu erhalten. Der Körper der Lehrerin wird zum Medium ,,indirekter Disziplinierung“, wie sie von Langer beschrieben worden ist (Langer 2008; Langer u. a. 2015). Bei einer indirekten Form der Disziplinierung soll Fremddisziplinierung vermieden werden. Anstatt direkt ermahnend oder strafend auf die Lernenden einzuwirken, wird versucht, über das Vorbild eines disziplinierten Lehrkörpers die Selbstdisziplin auf die Lernenden zu übertragen. Diese Strategie spiegelt sich im Interview mit L1. Das Ruhig-Halten des eigenen Körpers und die Neutralisierung oder Kanalisierung unwillkürlicher Bewegungsimpulse sind dabei zentral, der Leib oder die eigene leibliche Verfasstheit sollen 
ausgeblendet, der Lehrerinnenkörper medial genutzt und im Dienste der Herstellung oder Aufrechterhaltung einer sozialen Ordnung eingesetzt werden. Letztere wird dabei nicht in Frage gestellt, sondern vielmehr als sinnvoll vorausgesetzt. Der gezielte Einsatz körperlicher Haltungen und Gesten ist Teil feldspezifischer Verhaltensweisen im Umgang mit Unterrichtsstörungen. Die Perspektive, die L1 auf ihren eigenen Lehrerinnenkörper und den Umgang mit Unterrichtsstörungen einnimmt, ähnelt in gewisser Weise derjenigen, welche Pille bei Referendaren beschrieben hat (Pille 2009): mit dem bewussten, feldspezifischen Gebrauch des eigenen Körpers im Unterricht allgemein, insbesondere aber zur Herstellung von Ruhe, üben die Referendare nicht nur unterrichtliche Praktiken ein, sondern bilden auch eine Akzeptanz der sozialen Ordnung, die mit diesen Praktiken vollzogen wird, aus. Die Ordnung als solche wird dabei nicht in Frage gestellt, sondern immer wieder performativ hergestellt.

Im Vergleich zu L1 spielt bei L2 die Vorstellung eines Lehrkörpers, der als Medium fungiert, eine untergeordnete Rolle. In den Fokus stellt L2 nicht den eigenen und damit den Lehrerinnenkörper, sondern den Körper der Kinder, den sie im Hinblick auf deren körperliche Verfassung zu lesen versucht. In ihren Videobesprechungen rückt sie die Leiblichkeit bzw. das Leib-Sein der Kinder ins Zentrum. Die exakten Beschreibungen bzw. Beobachtungen der leiblichen Verfasstheit von Lernenden führen bei L2 in die Reflexion. Zum einen überdenkt sie ihre Unterrichtsplanung und ihr Bemühen, während des Unterrichts trotz Unruhen nicht von ihrem Programm abzuweichen. Dabei stellt sie auch institutionell verankerte Normvorstellungen, wie einen störungsfreien Unterricht, in Frage. Zwischen ihrem Auftrag als Lehrperson, planmäßig eine Unterrichtssequenz durch- und aufzuführen, und den Reaktionen und Bedürfnissen derjenigen, welche als stumme Mitspielende in diesem Plan vorgemerkt sind, klafft offensichtlich ein Widerspruch. Diesen - strukturellen - Widerspruch entdeckt sie, indem sie die Leiblichkeit der Lernenden in ihrer Widerständigkeit gegen die Inanspruchnahme durch die schulische Ordnung wahrnimmt und deutet.

Zum andern gelangt sie über die Betrachtung und die Analyse der leiblichen Verfasstheit eines individuellen Schülers zu einem überindividuellen, auch hier strukturellen Problem, demjenigen nämlich, als Lehrperson gleichzeitig verschiedenen Ansprüchen, demjenigen nach Individualisierung und demjenigen nach Gleichbehandlung, gerecht werden zu müssen.

Wie also, so lässt sich zum Schluss noch einmal fragen, tritt der Körper im Unterricht in Erscheinung? In welchem Verhältnis stehen die beiden Modalitäten Medialität und Leiblichkeit zueinander? In den beiden Fallrekonstruktionen ist die Beantwortung der Frage von der Wahrnehmung der Lehrpersonen abhängig. Im einen Fall wird der Körper primär in seiner Medialität wahrgenommen, im anderen in seiner Leiblichkeit. Die beiden Wahrnehmungsweisen, die anhand zweier unterschiedlicher Fälle ausdifferenziert worden sind, treten in Wirklichkeit wohl gemeinsam auf. Das Schwanken des Zuschauers bei der Wahrnehmung des Körpers des Schauspielers, das Umspringen der Wahrnehmung, welches Fischer-Lichte beim Zuschauer im Theater ausgemacht hat, dürfte auch für die Wahrnehmung des körpergebundenen Geschehens im Unterricht charakteristisch sein. Aber die zwei unterschiedlichen Perspektiven auf den Körper bzw. den Leib gehen - und das scheint uns entscheidend - Hand in Hand mit zwei unterschiedlichen Perspektiven auf die strukturellen Widersprüche, welchen die Körper 
der Lehrenden und Lernenden ausgesetzt sind: der Fokus auf den mediatisierten und instrumentalisierten Lehrkörper steht im Dienste einer reproduzierenden Aufführung des „Textes“, sprich der organisationsspezifischen Erwartungen und Anforderungen. Der Fokus auf die Leiblichkeit dagegen entdeckt die feldimmanenten Widersprüche und die Zumutungen, welche die tagtägliche Aufführung dieses ,Textes“ an die darin involvierten Subjekte bedeuten.

\section{Autorenangaben}

Dr. Regula Fankhauser

PH Bern

Institut für Weiterbildung und Medienbildung

Bereich Forschung und Entwicklung

regula.fankhauser@phbern.ch

\author{
Angela Kaspar \\ $\mathrm{PH}$ Bern \\ Institut für Forschung, Entwicklung und \\ Evaluation \\ angela.kaspar@phbern.ch
}

\section{Literatur}

Alkemeyer, Thomas (2009): Lernen und seine Körper. Habitusformungen und -umformungen in Bildungspraktiken. In: Friebertshäuser, B./Rieger-Ladich, M./Wigger, L. (Hrsg.): Reflexive Erziehungswissenschaft. Forschungsperspektiven im Anschluss an Pierre Bourdieu. Wiesbaden: Verlag für Sozialwissenschaften, S. 119-140.

Alkemeyer, Thomas/Pille, Thomas (2008): Schule und ihre Lehrkörper. Das Referendariat als Trainingsprozess. In: Zeitschrift für Soziologie der Erziehung und Sozialisation 28, 2, S. 137-154. [https://we.riseup.net/assets/75051/versions/1/alkemeyer-lehrkoerper.pdf], Zugriff am 15.01.

Breidenstein, Georg (2010): Überlegungen zu einer Theorie des Unterrichts. In: Zeitschrift für Pädagogik 56, 6, S. 869-887.

Csordas, Thomas J. (Hrsg.) (1994): Embodiment and Experience. The existential ground of culture and self. Cambridge: University Press.

Dirksmeier, Peter/Helbrecht, Ilse (2008): Time, Non-representational Theory and the "Performative Turn" - Towards a New Methodology in Qualitative Social Research. In: Forum Qualitative Sozialforschung 9, 2, Art. 55.

Fischer-Lichte, Erika (2004): Was verkörpert der Körper des Schauspielers? In: Krämer, S. (Hrsg.): Performativität und Medialität. München: Wilhelm Fink, S. 141-162.

Fischer-Lichte, Erika (2012): Performativität. Eine Einführung. Bielefeld: transcript.

Göhlich, Michael (2001): Performative Äusserungen. John L. Austins Begriff als Instrument erziehungswissenschaftlicher Forschung. In: Wulf. C./Göhlich, M. Zirfas, J. (Hrsg.): Grundlagen des Performativen. Eine Einführung in die Zusammenhänge von Sprache, Macht und Handeln. Weinheim und München: Juventa, S. 25-46.

Göhlich, Michael/Zirfas, Jörg (2001): Kommunikatives Handeln in der Lebenswelt. Die Theorie der performativen Einstellung von Jürgen Habermas. In: Wulf,C./Göhlich, M./Zirfas, J. (Hrsg.): Grundlagen des Performativen. Eine Einführung in die Zusammenhänge von Sprache, Macht und Handeln. Weinheim und München: Juventa, S. 47-73.

Hempfer, Klaus/Volbers, Jörg (Hrsg.) (2011): Theorien des Performativen. Sprache - Wissen Praxis. Eine kritische Bestandesaufnahme. Bielefeld: transcript. 
Henry, Stephen G./Fetters, Michael D. (2012): Video Elicitation Interviews: A Qualitative Research Method for Investigating Physician-Patient Interactions. In: Annals of Family Medicin,10,2, S. 118-125.

Hirschauer, Stefan (2008): Körper macht Wissen: für eine Somatisierung des Wissensbegriffs. In: Rehberg, K. S. (Hrsg.): Die Natur der Gesellschaft. Verhandlungen des 33. Kongresses der Deutschen Gesellschaft für Soziologie in Kassel. Frankfurt/M.: Campus, S. 974-984.

Kalthoff, Herbert/Kelle, Helga (2000): Pragmatik schulischer Ordnung. Zur Bedeutung von „Regeln“ im Schulalltag. In: Zeitschrift für Pädagogik 46, 5, S. 691-710.

Kloock, Daniela/Spahr, Angela (Hrsg.) (2012): Medientheorien. Eine Einführung. Paderborn: Fink.

Krämer, Sybille (1998): Das Medium als Spur und als Apparat. In: Krämer, S. (Hrsg.): Medien, Computer, Realität. Wirklichkeitsvorstellungen und Neue Medien. Frankfurt/M.: Suhrkamp, S. 73-94.

Krämer, Sybille (2004): Was haben ,Performativität ${ }^{`}$ und ,Medialität ${ }^{\star}$ miteinander zu tun? Plädoyer für eine in der ,Aisthetisierung' gründende Konzeption des Performativen. In: Krämer, S. (Hrsg.): Performativität und Medialität. München: Wilhelm Fink, S. 13-32.

Langer, Antje (2008): Disziplinieren und entspannen. Körper in der Schule-eine diskursanalytische Ethnographie. Bielefeld: transcript.

Langer, Antje (2011): „Körperbewusste Schule“? Funktionen und Adressierungen von pädagogischen Körperkonzepten. In: Keller, R./Meuser, M. (Hrsg.): Körperwissen. Wiesbaden: Verlag für Sozialwissenschaften, S. 317-334.

Langer, Antje/Richter, Sophia (2015): Disziplin ohne Disziplinierung. Zur diskursanalytischen Ethnographie eines ,Disziplin-Problems' von Schule und Pädagogik. In: Fegter, S./ Kessl, F./ Langer, A./Ott, M./Rothe, D./Wrana, D. (Hrsg.): Erziehungswissenschaftliche Diskursforschung. Empirische Analysen zu Bildungs- und Erziehungsverhältnissen. Wiesbaden: Verlag für Sozialwissenschaften, S. 211-229.

Mersch, Dieter (2006): Mediale Paradoxa. Zum Verhältnis von Kunst und Medien. Einleitung in eine negative Medienphilosophie. In: Sic et Non 6, 1, S. 1-14.

Meseth, Wolfgang/Proske, Matthias/Radtke, Frank-Olaf (2012): Kontrolliertes Laissez-faire. Auf dem Weg zu einer kontingenzgewärtigen Unterrichtstheorie. In: Zeitschrift für Pädagogik 58,2 , S. 223-241.

Pille, Thomas (2009): Organisierte Körper. Eine Ethnographie des Referendariats. In: Alkemeyer, T./Brümmer, K./Kodalle, R./Pille, T. (Hrsg.): Ordnung in Bewegung. Choreographien des Sozialen. Körper in Sport, Tanz, Arbeit und Bildung. Bielefeld: transcript, S. 161-178.

Plessner, Helmuth (1928): Die Stufen des Organischen und der Mensch. Einführung in die philosophische Anthropologie. Berlin: De Gruyter. 


\title{
Möglichkeitsräume des Performativen. Potenziale handlungsorientierter Methoden zur Förderung von Mündigkeit im Politikunterricht
}

\begin{abstract}
Zusammenfassung
Im fachdidaktischen Diskurs gilt Mündigkeit als ein ebenso konsensfähiges wie interpretationsbedürftiges Leitziel sozialwissenschaftlicher Bildung. Verstanden wird darunter vor allem die Fähigkeit zu eigenständig begründetem politischen Urteilen und Handeln, was die Fähigkeit der sozialen Perspektivübernahme wie der Reflexion handlungsleitender Emotionen mit einschließt. Vor diesem Hintergrund fragt der Beitrag nach den Potenzialen performativer Vollzüge von Perspektivwechseln beim Einsatz handlungsorientierter Methoden im Politikunterricht. Fokussiert wird dabei auf die Bedeutung von Ambiguitätserfahrungen und leibgebundenen Emotionen, die im Sinne des umrissenen Mündigkeitsverständnisses einer Reflexion bedürfen. Unter Rückgriff auf das theaterpädagogische Konzept der Differenzerfahrung des „Dazwischenstehens“ wird anhand eines Fallbeispiels exemplarisch herausgearbeitet, wie im performativen Nachvollzug einer sozialen Perspektivenübernahme emotionale Aspekte und Grenzen von Rationalität im Kontext politischer Urteile zugänglich gemacht werden können. Schlagwörter: Politikunterricht, politische Urteilsfähigkeit, handlungsorientierter Unterricht, Unterrichtsvideographie
\end{abstract}

\section{Potentials of action-oriented instruction methods to foster political judgment in civics courses}

Within the didactical discourse there is no doubt that the concept of "maturity" (Mündigkeit) is a core principle of civic education. As basic idea this concept includes competencies of autonomous and responsible judgment and participation as well as the ability to change perspectives and to thoroughly reflect on emotions in relation to politics. With this in mind, the article discusses the potentials of performing changes in perspective in the context of action-oriented instruction methods in civics courses. The focus is on experiencing ambiguity and emotions, which call for reflections in order to foster the competency of responsible political judgment. An exemplary case study borrows the concept of "being-in-between" from theatre education to demonstrate how emotions and bounded rationality in politics become evident in performing changes of perspective.

Keywords: civics courses, political judgment, action-oriented instruction, video studies

\section{Einleitung}

Ausgangspunkt des vorliegenden Beitrags ist ein im Kontext des Projekts „Level“1 an der Goethe-Universität Frankfurt am Main verfolgtes Forschungsvorhaben, das Mündigkeit als Leitziel sozialwissenschaftlicher Bildung fokussiert. Anhand videographier-

1 Das Projekt „Level - Lehrerbildung vernetzt entwickeln“ wird im Rahmen der gemeinsamen „Qualitätsoffensive Lehrerbildung“von Bund und Ländern aus Mitteln des Bundesministeriums für Bildung und Forschung gefördert. Dem Artikel gehen gemeinsame Vorarbeiten innerhalb des sozialwissenschaftlich-historischen Fächerverbundes voraus, für inhaltliche Anregungen ist insbesondere Maria Theresa Meßner und Tim Engartner sowie den Gutachter/innen und Herausgeber/innen des Beitrags zu danken. 
ter Unterrichtseinheiten sollen zentrale Aspekte der Förderung dieses Leitziels in Unterrichtsprozessen identifiziert, analysiert und interpretiert werden, um daraus didaktischmethodische Schlüsse für Lehrer/innenhandeln ableiten zu können. Unter Mündigkeit als Zielorientierung politischer Bildung wird vor allem die Fähigkeit zu eigenständig begründetem politischen Urteilen und Handeln verstanden, womit nicht nur Prinzipien der Rationalität, sondern auch die Fähigkeit der sozialen Perspektivenübernahme sowie die Reflexion handlungsleitender Emotionen angesprochen werden (2.). In dem Zusammenhang fragt der Beitrag nach möglichen Potenzialen performativer Bildungsprozesse beim Einsatz handlungsorientierter simulativer Methoden im Politikunterricht und greift dafür auf den theaterpädagogischen Begriff der Differenzerfahrung des „,Dazwischenstehens" zurück, der im Sinne einer für politisches Lernen bedeutsamen Ambiguitätserfahrung interpretiert wird (3.). Anhand eines Fallbeispiels wird daran anschließend exemplarisch herausgearbeitet, wie im performativen Nachvollzug einer sozialen Perspektivenübernahme emotionale Aspekte und Grenzen von Rationalität als leibgebundene in einem Erfahrungsmodus des „Dazwischen“ zugänglich gemacht werden können (4.), um aus diesen konzeptionellen Vorarbeiten abschließend eine Perspektive für das aktuelle Forschungsvorhaben zu entwickeln (5.).

\section{Mündigkeit als Leitziel Politischer Bildung}

Mit dem Begriff der Mündigkeit wird nicht nur ein erziehungswissenschaftlicher Leitbegriff, sondern zugleich eine - wenn nicht die - „Leitidee von Bildung und [...] Maxime politischer Bildung“" (Autorengruppe Fachdidaktik 2016: 13) angesprochen, die als mindestens ebenso interpretationsbedürftig wie konsensfähig gilt (ebd.). Im fachdidaktischen Selbstverständnis wird das Leitziel der Mündigkeit in aufklärerischer Tradition in der Regel mit dem Konzept einer rationalen politischen Urteilsfähigkeit verbunden (vgl. ebd.: 17; Henkenborg 2012: 28-29). Gleichwohl besteht auch im Hinblick auf diesen Begriff Klärungsbedarf, welche Arten von Urteilen darunter zu fassen sind und welche Rationalitätsdimensionen davon berührt werden (vgl. Henkenborg 2012). Ein politisches Urteil impliziert den Einbezug zugrunde liegender Wertorientierungen sowie die Fähigkeit, diese auch in kompensatorischer Weise gegeneinander abzuwägen und zu einer eigenständig begründeten Entscheidung zu gelangen (vgl. Henkenborg 2012; Juchler 2005a). Abgegrenzt wird es damit von einer an Kriterien der sachlichen Richtigkeit orientierten Analysekompetenz, mit der die Fähigkeit bezeichnet wird, politische Sachverhalte zu erfassen und in ihren Zusammenhängen zu erklären. Innerhalb des politikdidaktischen Diskurses entwikkelte sich dabei vor allem ein mit einem kognitiven Vernunftbegriff verbundenes Rationalitätsverständnis als herausragendes Merkmal politischer Urteile (vgl. Juchler 2005a: 63), was kritisch als „rationalistische Schlagseite“ (Weber 2016: 170) zentraler Begriffe der Politischen Bildung gekennzeichnet wird. Davon ausgehend werden hier zwei Aspekte skizziert, die im Diskurs um mündige politische Urteilsbildung bisweilen als Leerstellen beschrieben werden, um im Folgenden die Potenziale handlungsorientierter simulativer Unterrichtsmethoden im Hinblick auf diese Aspekte in den Blick nehmen zu können.

Angesprochen wird zum einen eine Vernachlässigung der Anteile von Emotionen bei der politischen Urteilsbildung und beim politischen Handeln (z. B. Breit 1991: 69). 
Dabei leisten Konflikte um sowie Reflexionen über allgemeinverbindliche (moralische) Grundwerte einer Gesellschaft einen wesentlichen Beitrag zur Befähigung zur politischen Urteilsbildung (Reinhardt 2005a: 368-369), in deren Rahmen auch die handlungsleitende Bedeutung von Emotionen zu diskutieren ist. Eine demokratische Argumentationskultur, die nicht nur, ,gleichwertige Teilhabe und wechselseitige Anerkennung der Diskurspartner“, sondern auch die „Akzeptanz von Differenz des Anderen und NichtEigenen“ (Oeftering/Uhl 2010: 68) voraussetzt, geht dabei in ihren Anforderungen noch über die Reflexion eigener emotionaler Betroffenheit hinaus. Angesichts der in demokratischen Systemen vorzufindenden Pluralität von Meinungen (Juchler 2005b: 121122) wird eine zunehmende Komplexität der Ausgangssituationen politischer Urteilsbildung konstatiert und allgemein Zweifel an umfassender und konsensfähiger Rationalität geäußert (Schröder 2015). Als Korrektiv wird mit dem Konzept der erweiterten Denkungsart eine Form reflektierenden Urteilens vorgeschlagen (ebd.: 147-148; Juchler 2005b: 141), die mit der Fähigkeit zur sozialen Perspektivenübernahme im Sinne Kohlbergs verknüpft wird und so eine Verbindung der, gefühlsmäßige[n] Wertorientierung der Schüler mit politischem Denken“ (Breit 1991: 63) herstellt.

\section{Performative Bildungsprozesse und Potenziale handlungsorientierter Methoden im Politikunterricht}

Ausgehend von der skizzierten Grundüberlegung, dass die Reflexion handlungsleitender Emotionen, die Einsicht in die Grenzen von Rationalität und die Fähigkeit zur sozialen Perspektivenübernahme als Bestandteile einer mündigen Urteilsbildung im Politikunterricht zu fördern sind, wird von einem besonderen Potenzial handlungsorientierter Unterrichtsmethoden ausgegangen (Massing 1998: 8). Handlungsorientierung kann ähnlich dem Begriff der Mündigkeit einerseits als ein Leitbegriff der Didaktik bezeichnet werden, andererseits läuft der Begriff in seiner konzeptionellen Diffusität und oftmals in Verbindung mit einer emphatisch-naiven Verwendungsweise des Handlungsbegriffs Gefahr, auf eine Leerformel reduziert zu werden (Grammes 1999: 212). Nach einer gängigen Klassifizierung können drei als Reales Handeln, Simulatives Handeln und Produktives Gestalten bezeichnete Verfahren handlungsorientierter Unterrichtsmethoden in schulischen Kontexten unterschieden werden (Reinhardt 2005b: 146), wobei dieser Beitrag vor allem nach den Potenzialen simulativer Unterrichtsmethoden, wie z. B. Rollen- oder Planspielen, fragt. Zwar kann mit Blick auf die erweiterte Denkungsart, die im vorangegangenen Abschnitt gegenüber einer einseitig rationalen Sichtweise als Korrektiv in Stellung gebrachte wurde, argumentiert werden, dass diese auf die Fähigkeit zielt, unterschiedliche eigene Perspektiven zu entwickeln, einzunehmen und daran das eigene Urteil zu schärfen. Simulativen Unterrichtsmethoden wäre in dem Zusammenhang entgegenzuhalten, dass Perspektiven anderer Menschen nur in direkter Konfrontation und nicht durch fiktives Hineinversetzen nachvollzogen werden können, sondern dieses vielmehr die „Gefahr der Konstruktion und Verstetigung von Vor- und Fehlurteilen“ (Schröder 2015: 148) durch stereotype Darstellungen birgt. Demgegenüber möchte dieser Beitrag nun vor allem die Potenziale der performativen Prozesse im Zuge solcher Unterrichtsmethoden in den Fokus der Aufmerksamkeit rücken, um 
angesichts der skizzierten Herausforderungen nach Möglichkeiten der Förderung der Fähigkeit zur sozialen Perspektivenübernahme im Sinne des umrissenen Mündigkeitsverständnisses zu fragen.

\subsection{Perspektivwechsel im Kontext simulativer Unterrichtsmethoden}

Über die von Massing beschriebenen Potenziale im Hinblick auf die Aneignung nicht unmittelbar sach- und gegenstandsbezogener Fähigkeiten und Fertigkeiten hinaus (1998: 8) steht an dieser Stelle vor allem der performative Vollzug eines Perspektivwechsels beim Einsatz simulativer Unterrichtsmethoden im Fokus des Interesses. Ausgegangen wird von einem Lernen in Interaktion (Reinhardt 2005b: 147), wobei die Übernahme einer Rolle in einer Simulation von den Lernenden verlangt, sich sowohl in die ,eigene Position als auch in die der ,anderen' hineinversetzen zu können, um sich produktiv in diese simulierte Interaktion einbringen zu können (Engartner et al. 2015: 198-200). Von besonderer Bedeutung ist dabei der Charakter des Als-Ob-Handelns (Reinhardt 2005b: 146), der die „Möglichkeit realitätsnaher Probehandlungen“ eröffnet, sodass „Konsequenzen von Handlungen [...] ohne reale Auswirkungen erfahrbar“ werden können (Engartner et al. 2015: 197; Herv. i. O.). Dass die simulierten Handlungen im Spielverlauf allerdings nur im Verhältnis zu realem politischem Handeln als Probehandlungen ohne reale Auswirkungen beschrieben werden können, innerhalb des übergeordneten und mit Beurteilungsprozessen verbundenen Handlungsrahmens Unterricht für die Schüler/innen aber durchaus reale und nicht unbedingt folgenlose Handlungen darstellen, verweist auf eine im Fachdiskurs nur selten thematisierte Differenzierung (z.B. Birgmayer 2011). Entsprechend wird das für die politische Urteilsbildung als relevant erachtete Potenzial des performativen Vollzugs eines Perspektivwechsels an dieser Stelle zunächst in Absehung von dieser Einschränkung ausgeführt, um im Weiteren der offenen Frage nachzugehen, ob und inwieweit dieser übergeordnete Handlungsrahmen zumindest zu Ambivalenzen im Agieren in den Rollen wie in der Verkörperung der Positionen in der Interaktion führt.

Der erwähnten „Gefahr der Konstruktion und Verstetigung von Vor- und Fehlurteilen“" (Schröder 2015: 148) soll vor allem mittels eines dezidierten inhaltlichen Sachbezugs entgegengearbeitet werden, der durch die Einnahme ,unterschiedliche[r] Perspektiven auf gesellschaftliche und politische Problemstellungen" sowie durch die darauf aufbauende Auseinandersetzung mit „Konflikten, Ambiguitäten und Vorurteilen“ (ebd.: 200; Herv. i. O.) hergestellt wird. Ein besonderer Stellenwert kommt in diesem Zusammenhang der an die Simulationsphase anschließenden Auswertungsphase zu, in der eine umfassende Reflexion der Spielhandlung und möglicher in ihr enthaltener Verkürzungen der Realität erfolgt (Engartner et al. 2015: 203; Grammes 1999: 213). Wenn das dabei zu beobachtende Zusammenspiel von Emotionalität und Rationalität als affektive Lerndimension bezeichnet wird, sind damit in der Regel motivationale Aspekte oder der Erwerb sozialkommunikativer Fähigkeiten und Fertigkeiten gemeint. Allerdings kann dieser affektive Aspekt darüber hinaus insofern als Anbahnung einer „Denkoperation“ (Breit 1991: 63) gedacht werden, als dass an die auch leiblich zu vollziehende soziale Perspektivenübernahme Erfahrungen gebunden sind, die in der Reflexion politisches Lernen ermöglichen können (Engartner et al. 2015: 203). 


\subsection{Perspektivwechsel im Verhältnis von Mimesis und Performativität}

Mit dem Fokus auf den der Reflexion vorausgehenden performativen Vollzug einer sozialen Perspektivenübernahme wird zunächst an Grammes angeschlossen, der Simulationen als „Re-Präsentationen von Wirklichkeit“ im Sinne „mimetische[n] Nachbilden[s] und Ausprobieren[s] sozialer Vorgänge“ (1999: 216) bezeichnet. Es kann dabei insofern von einem mimetischen Verhältnis ,zwischen der internen Ordnung des Spiels und der Ordnung der Gesellschaft, in der es inszeniert und aufgeführt wird" (Wulf 2005b: 16) ausgegangen werden, als dass die Handlungen des Spiels Elemente und Strukturen der gesellschaftlichen Ordnung aufnehmen, diese in der Inszenierung und Aufführung des Spiels sichtbar machen, sie potenziell verändern und auf sie zurückwirken können (ebd.). Die dabei vollzogenen, als mimetisch beschriebenen Prozesse lassen sich im Kontext der Wahrnehmung von und der Teilnahme an szenischen Aufführungen sozialer Handlungen sowie ritueller Szenen verorten, die sich körperlich-aisthetisch vollziehen (Wulf 2005a: 94-95). Erworben bzw. aktualisiert wird dabei eine spezifische Art mimetischen Wissens, das als atheoretisches und reflexives charakterisiert wird und eine grundlegende Voraussetzung sozialen Handelns bildet. Mimesis wird in diesem Kontext als eine kreative, menschliche Fähigkeit verstanden, mit deren Hilfe über die lediglich kopierende Imitation eines Vorbilds hinaus Neues geschaffen wird, wobei eine Beziehung zur vorgefundenen Wirklichkeit bzw. zu einer vorgestellten Welt grundlegend für den Mimesis-Begriff bleibt (ebd.: 72f.). Die Performativität körperlicher Aufführungen konstituiert sich in so verstandenen mimetischen Prozessen und zeichnet sich durch die damit einhergehende Hervorbringung von etwas Neuem aus, das erst im Vollzug als Einmaliges hergestellt wird (Wulf 2005a: 70; Wulf/ Zirfas 2007: 31).

Da der Als-ob-Charakter und möglicherweise im Spiel gestellte Leistungsanforderungen nicht reibungslos ineinander aufgehen (Wulf 2005b: 20), kommt dem Spiel ein agonaler Charakter zu, der in Bezug auf simulative Unterrichtsmethoden in besonderer Weise zu denken ist: Insofern der übergeordnete Handlungsrahmen selbst ein mit Leistungsanforderungen und -bewertungen verknüpftes Szenario darstellt, ist von Ambivalenzen im Verhältnis von Spiel und Unterricht auszugehen, die es auch mit Blick auf die Förderung politischer Urteilsbildung zu reflektieren gilt.

\subsection{Im „Dazwischen“ der Verkörperung - Möglichkeitsräume des Performativen}

In der Performativität sozialen Handelns in seinen kulturellen, sprachlichen und ästhetischen Dimensionen wird - so die leitende Annahme - praktisches performatives Wissen mimetisch erworben. In der spielerischen Simulation kommt die Körperlichkeit der Spielenden in einer besonderen Weise, nämlich in ihrer Verdopplung, zum Tragen (Wulf 2005b: 16), was in der Theaterwissenschaft mit der Kategorie der „Verkörperung“ beschrieben wird (Fischer-Lichte 2001: 17). So besteht zwar eine Differenz zwischen Subjekt und verkörperter Simulation, die dabei gemachten Erfahrungen sind dennoch als an den Leib als „Ausdrucksorgan“ und „Empfindungsträger“ (Westphal 2005: 109; Herv. 
i. O.) gebundene und damit körperliche zu verstehen (Wulf 2005a: 61) und wirken als solche wieder auf das Subjekt zurück (ebd.: 95).

Auch wenn mit Blick auf das Theater als eine ästhetische Gestaltungspraxis sowie hinsichtlich der Prozesse künstlerischer Ausdrucksgestaltung (Fischer-Lichte 2004) von einer Differenz zwischen theatralem Spiel und der Anwendung handlungsorientierter Unterrichtsmethoden in Form von Simulationen auszugehen ist (Wulf 2005a: 11; Engartner et al. 2015: 198), wird hier auf die Gemeinsamkeit der mit beidem verbundenen Differenzerfahrung des „Dazwischenstehens“ fokussiert, die sich auch als für politisches Lernen bedeutsame Ambiguitätserfahrung beschreiben lässt (Hentschel 2010: 238; Engartner et al. 2015: 200). Dabei wird davon ausgegangen, dass „Ambiguitätstoleranz“ als die „Fähigkeit, bestehende und eventuell gar nicht lösbare Konflikte und Doppeldeutigkeiten auszuhalten sowie alternative Meinungen bestehen zu lassen" (Engartner et al. 2015: 200), durch die in Simulationen zu vollziehende soziale Perspektivenübernahme gefördert wird (ebd.). In Bezug auf das politische Handeln innerhalb der Simulation wird zum anderen von der Annahme ausgegangen, dass das Experimentierfeld einer handlungsentlasteten Spielsituation im Unterschied zur sozialen Praxis einen Möglichkeitsraum zur Erprobung neuer Perspektiven sowie der Neukonstruktion von Zusammenhängen und zur Kreation neuer Wahrnehmungsweisen ermöglicht (Westphal 2005: 119). Der Erfahrungsmodus des „Dazwischen“ (Hentschel 2010: 160161 ) ist demnach in diesen unterschiedlichen Relationen zu denken.

Die Aufmerksamkeit in der exemplarischen Analyse des Fallbeispiels soll sich dabei vorrangig auf meist vernachlässigte Aspekte sprachlicher und körperlicher Performativität in der Ausgestaltung sozialer Beziehungen (Wulf 2005a: 12) sowie auf die mit mimetischen Prozessen einhergehenden emotionalen Erfahrungen (ebd.: 33) richten. Nachgegangen werden soll der Frage, ob das Fallbeispiel Anhaltspunkte dafür gibt, dass sich in der Simulation im Erfahrungsmodus des „Dazwischen“ neue zunächst performativ ausgestaltete Möglichkeitsräume eröffnen, die in der anschließenden Reflexion wiederum so erschlossen werden können, dass sie einer reflexiv-anspruchsvollen Form politischen Urteilens zugänglich gemacht werden können. Zu fragen wäre also, ob mit der sozialen Perspektivenübernahme einhergehende Erfahrungen der Grenzen von Rationalität zu einer angemessenen Anerkennung pluraler Sichtweisen und darüber hinausgehender emotionaler Aspekte bei der eigenen Urteilsbildung führen. Ausgegangen wird dabei zunächst von der These, dass sich in der Verkörperung einer eingenommenen Rolle neben der bewussten argumentativen Ausgestaltung der inhaltlichen Position mimetische Prozesse vollziehen, die in Beziehung zu vorausgehenden (Welt-)Erfahrungen zu interpretieren sind, eigenen Dynamiken unterliegen und in diesen wiederum auf die Verkörperung und die interaktiven Prozesse in der Simulation rückwirken. 


\section{4. „Du kannst das doch nicht wegschmeißen wie einen Brocken Fleisch.“ - Das Fallbeispiel einer Talkshow zum $\S 218$ StGB}

Bei dem herangezogenen Fallbeispiel handelt es sich um eine Unterrichtseinheit zur politischen Auseinandersetzung um den $\S 218$ StGB (Schwangerschaftsabbruch), in der das Szenario der Talkshow zum Einsatz kommt. Die Unterrichtseinheit wurde am 08.01.1993 in einer elften Klasse in Berlin im Rahmen eines Weiterbildungskurses durchgeführt und aufgezeichnet. ${ }^{2}$ Thematisch wird eine zu diesem Zeitpunkt gesellschaftlich breit geführte Debatte aufgegriffen; die gewählte Methode Talkshow wird im didaktischen Diskurs als ambivalent eingeschätzt: Dem Potential, unterschiedliche Positionen einer politischen Debatte prägnant herausarbeiten und darstellen zu können, wird die Gefahr vordergründiger Selbstdarstellungen und beliebiger Meinungsdeklarationen gegenübergestellt (Massing 1998: 42).

\subsection{Methodische Überlegungen zur Analyse und Interpretation des Fallbeispiels}

Davon ausgehend, dass Kommunikation das zentrale Medium darstellt, in dem in „ko-produktive[r] Auseinandersetzung aller Beteiligten“ (Meseth/Proske/Radtke 2012: 213) Unterrichtsgegenstände konstituiert sowie Aushandlungsprozesse über deren Sinn und Bedeutung vollzogen werden, setzen die Analyse und Interpretation auch auf dieser Ebene an (Jehle/Blessing 2014). Dabei soll weniger die inhaltlich-argumentative Ausgestaltung der sozialen Perspektivenübernahme als deren mimetischer wie performativer Vollzug in den Blick genommen werden. Im Hinblick auf die Zusammenhänge von Sprache und Performanz sind sprachliche Äußerungen dabei auch in ihrem deklarativen Charakter und mit Blick auf die in ihnen enthaltenen Selbstdeutungen von Bedeutung (Wulf 2005a: 87-89). Die Rekonstruktion der kommunikativen und performativen Prozesse im Zuge von Perspektivenübernahmen in der Durchführungs- und Auswertungsphase der Talkshow greift dabei auf Grundannahmen und Verfahren der ethnomethodologischen Konversationsanalyse zurück (Breidenstein/Tyagunova 2012). Die gesamte Aufzeichnung wurde in Anlehnung an Verfahren ethnographischer Mikroanalysen Erickson 1992; Herrle 2013) in Sequenzen unterteilt und Feinanalysen unterzogen. Da aufgrund des begrenzten Umfangs dieses Beitrags die einzelnen Analyseschritte nicht vollumfänglich dargelegt werden können, beschränkt sich die Darstellung

2 Die Aufzeichnung gehört zu einem Bestand historischer Videoaufzeichnungen von Unterricht aus der Bundesrepublik Deutschland, die im Rahmen eines Forschungsprojektes an der Universität Wien in Kooperation mit dem Deutschen Institut für Internationale Pädagogische Forschung (DIPF) in einer Online-Datenbank der wissenschaftlichen Forschung zugänglich gemacht wurden (Schluß/Jehle 2013): https://www.forschungsdaten-bildung. de/studiendetails.php?id=116 (20.07.2017). Das anonymisierte Transkript der hier diskutierten Unterrichtsaufzeichnung (Aufzeichnungseinheit 1993) wird ebenfalls in dieser Datenbank zur Verfügung gestellt. Im Projekt „Level“ wurden diese und andere Aufzeichnungen für konzeptionelle Vorarbeiten innerhalb des sozialwissenschaftlich-historischen Fächerverbunds zur Analyse der Förderung von Mündigkeit im sozialwissenschaftlichen Unterricht mittels spezifischer Unterrichtsmethoden genutzt. 
auf Auffälligkeiten im Diskussionsverlauf, dessen spezifische Dynamik anhand einer ausgewählten Sequenz exemplarisch nachvollzogen wird. Eine performative Sichtweise ist dabei insbesondere an „Prozesse[n] der Interaktion“, „dramaturgische[n] Handlungsvollzügen“ sowie der „Körperlichkeit“ (Wulf/Zirfas 2007: 9) der Akteure interessiert und behält sich im Rückgriff auf ethnographische und interaktionistische Modelle eine gewisse - nicht mit Beliebigkeit zu verwechselnde - methodische Offenheit vor. Besondere Aufmerksamkeit gilt dabei der jeweiligen Situationsspezifik, die sich wiederum aus der Offenheit handlungsorientierter Methoden ergibt (Blötz 2015: 230). Die aus der spezifischen Situation entstehenden Möglichkeitsräume werden dabei im Erfahrungsmodus des „Dazwischen“ performativ ausgestaltet. Insbesondere sollen die der Unterrichtsmethode Talkshow zugeschriebenen Dynamiken (Massing 1998: 43) in ihrem performativen Vollzug im Fokus des Interesses stehen, wofür auf den aus der dokumentarischen Methode stammenden Begriff der „Fokussierungsmetapher“ zurückgegriffen wird. Mit diesem werden Passagen in Diskursverläufen bezeichnet, die sich „durch einen gemeinsamen Rhythmus und somit durch eine ,interaktive Dichte" auszeichnen“. Einher geht das ,,in der Regel mit einer ,metaphorischen Dichte“, einer Dichte hinsichtlich der Bildhaftigkeit und Detailliertheit der Darstellung“ (Bohnsack/Przyborski 2010: 234), ohne dass damit bereits die Annahme eines konjunktiven Erfahrungsraums im Sinne der dokumentarischen Methode gesetzt sein muss (ebd.: 246).

\subsection{Exemplarische Analyse einer ausgewählten Sequenz}

Für die exemplarische Analyse wurde eine Sequenz ausgewählt, die sich nicht nur durch eine hohe interaktive Dichte auszeichnet, sondern anhand derer sich eine Reihe von Aspekten ausweisen lassen, die als charakteristisch für den gesamten Diskussionsverlauf bezeichnet werden können. Die Sequenz ist am Beginn des letzten Drittels der 45-minütigen Simulationsphase zu verorten. Nachdem die Teilnehmenden in einer ersten Phase vergleichsweise längere Beiträge in einer durch den Moderator geordneten Form vorgebracht haben, gewinnt das Gespräch an Dynamik, als sich Sm11 in der Rolle des Kirchenvertreters mit der entschiedenen Position einbringt, dass Schwangerschaftsabbruch Mord und gegen den Willen Gottes sei. Mit zahlreichen Unterbrechungen und teilweise erheblichen Steigerungen der Lautstärke ist eine deutliche Zunahme an Fokussierungsmetaphern zu verzeichnen, was auf einen hohen Grad an Involviertheit der Akteure verweist. Bis hierhin wurden in der Diskussion bereits Fragen des beginnenden Lebens und der Definition von Mord sowie der sozialen Verantwortung für die Kinderversorgung und deren Finanzierung in der Diskussion bearbeitet. In der Rolle eines Vertreters des Gruppenantrages, der 1992 als fraktionsübergreifender Kompromiss in den Bundestag eingebracht wurde und sich für eine Fristenregelung ausspricht, greift $\mathrm{Sm} 3$ erneut die Frage auf, wann menschliches Leben beginne, womit die in der ausgewählten Sequenz dokumentierte Auseinandersetzung zwischen Sm3 und Sm11 beginnt (Abb. 1). 


\begin{tabular}{|c|c|c|c|c|}
\hline Zeit & Kamera & $\begin{array}{l}\text { Spre- } \\
\text { cher } \\
\text { in }\end{array}$ & Audio-Ebene & Video-Ebene \\
\hline $00: 49: 00$ & S.I. & $\begin{array}{l}\text { Sm? } \\
\text { Sm3 } \\
\text { Sm11 } \\
\text { Sm3 } \\
\text { Sm11 } \\
\text { Sm3 } \\
\text { Sm11 } \\
\text { Sus } \\
\text { Sm11 } \\
\text { Sm? } \\
\text { Sm11 } \\
\text { Sm? } \\
\text { Sm? } \\
\text { Sus } \\
\text { Sm? }\end{array}$ & 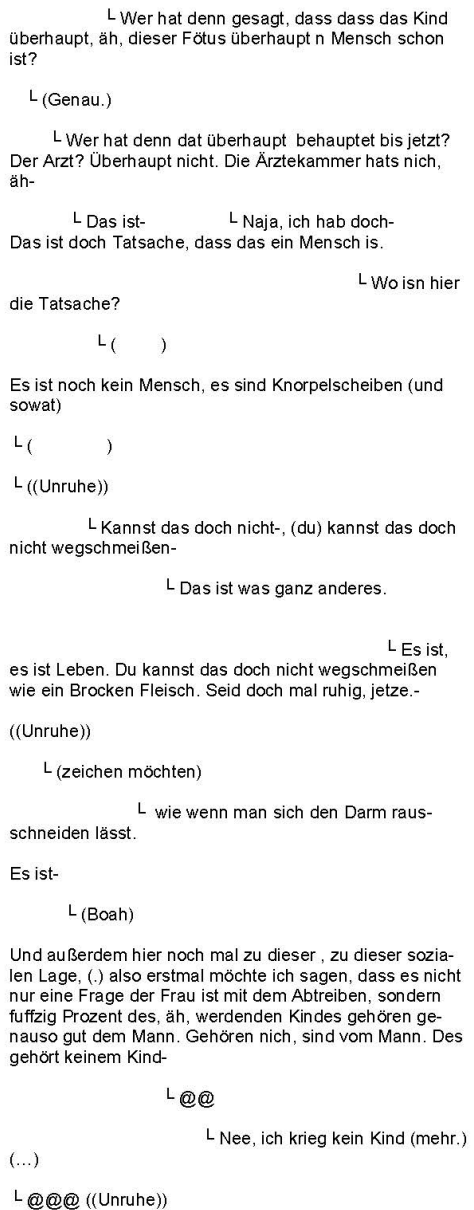 & $\begin{array}{l}\text { Sm11 sieht in Unterlagen } \\
\text { Sm11 sieht auf, gestikuliert mit Stift in rechter } \\
\text { Hand, sieht nach rechts, lässt rechte Hand sinken, } \\
\text { gestikuliert mit linker Hand } \\
\text { Sm11 sieht in seine Unterlagen, sieht auf, sieht } \\
\text { nach rechts } \\
\text { Sm11 sieht Richtung Klasse } \\
\text { Sm11 sieht in seine Unterlagen }\end{array}$ \\
\hline
\end{tabular}

Abb. 1: Transkriptauszug (Aufzeichnungseinheit 1993: 00:48:56-00:49:52)3

$\mathrm{Zu}$ beobachten ist dabei ein zunehmendes, sich auch körperlich ausdrückendes In-derRolle-Sein, wie es in den illustrierenden Standbildern zum Ausdruck kommt: Stellte das erste Statement von Sm3 vor allem ein Verlesen der vorbereiteten Position dar, bei dem er den Blick auf die Unterlagen gerichtet hält und sich gewissermaßen an diesen ,festhält"“ (Abb. 2), spricht er jetzt frei und agiert Raum einnehmend mit dem Oberkörper. Als er seine Frage nach dem Beginn des Lebens an Sm11 richtet, beugt er sich so weit

3 Legende der verwendeten Abkürzungen und Transkriptionszeichen: $\mathrm{Lw}=$ Lehrerin $/ \mathrm{Sw}=$ Schülerin / Sm = Schüler; ( ) = unverständlich; $(x x x)=$ leise oder schwer verständlich; L = Überlappung; @ = Lachen oder lachend gesprochen; (.) = Pause (1s); (..)= Pause (2s); S.r. = Schwenk nach rechts; S.I. = Schwenk nach links. 
nach vorne, dass er die Grenze des Nahbereichs zu dem neben ihm sitzenden Sm5 überschreitet (Abb. 4). Während Sm11 bei seinem zeitgleich vorgetragenen Einwand nicht zu sehen ist, richtet Sm3 sich im Weitersprechen kurz auf und weist mit einer Geste auf den rechts neben ihm sitzenden Sm4 in der Rolle des Arztes, um sich dann mit der Nachfrage „Wo isn hier die Tatsache?“ wieder nach vorne in Richtung Sm11 zu beugen. Die erste Erwiderung von Sm11 ist aufgrund der Unruhe in der Klasse nicht zu verstehen; bei seiner Äußerung ,,(du) kannst das doch nicht wegschmeißen“ wendet er sich mit dem Oberkörper der Klasse zu, womit er möglicherweise auf von dort kommende Einwände reagiert (Abb. 5). Dabei lassen sich auch bei Sm11 deutliche Differenzen in der Körperhaltung im Vergleich zu seinem ersten in der Diskussion vorgebrachten Statement beobachten: In der Positionierung seines Oberkörpers ist er zunächst vor allem der Gesprächsrunde zugewandt, der Blick ist bei weitestgehend ruhiger Körperhaltung größtenteils auf die Unterlagen gerichtet (Abb. 3). Im Verlauf der Diskussion weitet er den Aktionsradius, den er mit seinem Oberkörper einnimmt, mit raumgreifenden Gesten aus und interagiert verbal wie nonverbal ebenso mit dem Publikum.



Abb. 2: Standbild 00:20:28

(Aufzeichnungseinheit 1993)

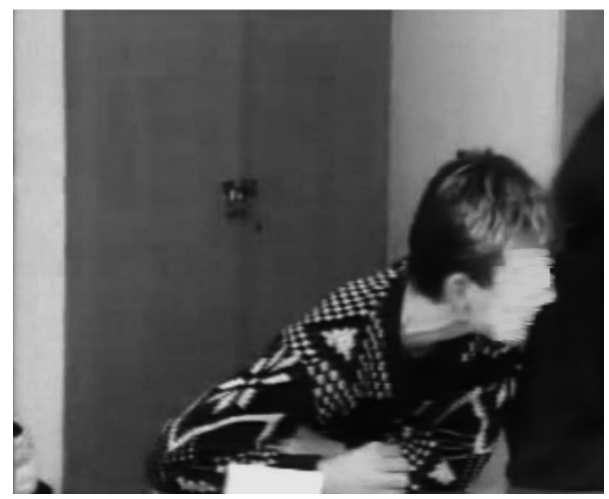

Abb. 4: Standbild 00:49:02 (ebd.)

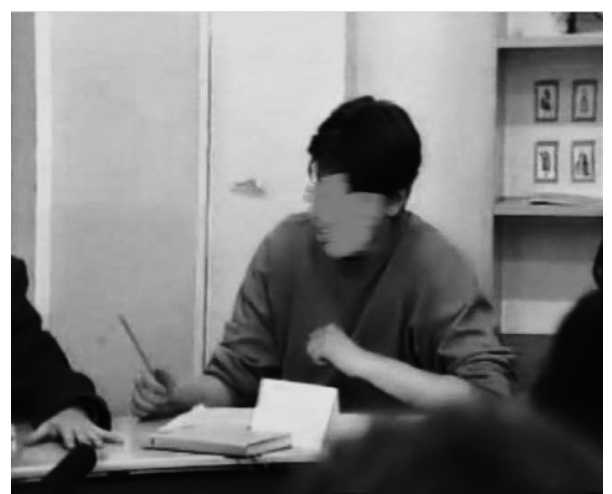

Abb. 3: Standbild 00:32:42 (ebd.)

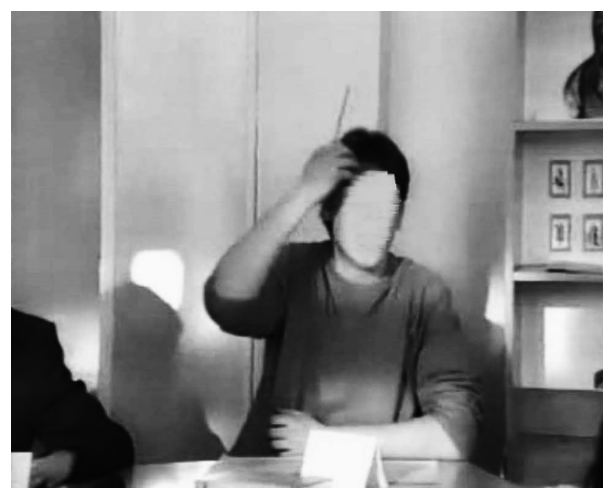

Abb. 5: Standbild 00:49:19 (ebd.) 
Diese Haltungen und Gesten lassen sich als Verkörperungen emotionaler Ausdrucksformen auffassen, die soziale Beziehungen konstituieren, Beziehungsgefüge regulieren (Kellermann 2012: 100), innerhalb dieser ,,als Körper-Ausdrücke und -Eindrücke wechselseitig zirkulieren" (ebd.: 102) und wiederum zurückwirken. Die im Rahmen der Talkshow widerstreitenden Positionen bringen die Schüler Sm3 und Sm11 in ihren Positionierungen zueinander auch expressiv als konkurrierende zum Ausdruck. Betrachtet man diese interaktiven Positionierungen als eine Aufführung von Statusrepräsentationen (ebd.: 109), kann die zunehmende Vehemenz, mit der die Rollenspieler/innen ihre Positionen vertreten, als Resultat einer wechselseitigen Verstärkung von Körper-Ausdrücken und -Eindrücken, interpretiert werden. Gebündelt werden diese Beobachtungen in der These, dass die in der Verkörperung der zum Ausdruck gebrachten Emotionen innerhalb des interaktiv hergestellten Beziehungsgefüges zirkulieren, dabei Transformationsprozesse durchlaufen und wiederum als leibgebundene in neuer Intensität erfahren werden können.

Nachdem im Verlauf der Diskussion inzwischen die meisten der vorbereiteten Argumente eingebracht worden sind, lassen sich in der Konfrontation der Positionen auch Veränderungen auf der Ebene der sprachlichen Performanz beobachten: So bemüht sich Sm3 in der Rolle eines Bundestagsabgeordneten um eine gewählte Ausdrucksweise und sachliche Begründungen, nach der eine Äußerung wie „,es sind Knorpelscheiben (und sowat)“ als der Rolle nicht angemessen zu klassifizieren wäre. Zugleich wird aber gerade in dieser Formulierung die in der Äußerung enthaltene Abwertung performativ hervorgebracht, was wiederum auf das In-der-Rolle-Sein von Sm3 verweist. Auch Sm11 kann an dieser Stelle keine inhaltlich stichhaltigen Argumente vorbringen, sodass er im Anschluss an seinen Versuch, für Ruhe zu sorgen, nach einem Blick in seine Unterlagen einen Themenwechsel versucht. Der zuvor in mehreren Anläufen eingebrachte Ausruf „Du kannst das doch nicht wegschmeißen wie einen Brocken Fleisch“ adressiert vielmehr auf emotionaler Ebene dahinter stehende Wertorientierungen. Derartige Ambivalenzen im sprachlichen Agieren in der Rolle können einerseits als ein Überspielen von Defiziten auf der inhaltlichen Ebene interpretiert werden, andererseits verweisen sie auf mögliche Grenzen argumentativer Begründungen und lassen auf der performativen Ebene Zusammenhänge von Wertorientierungen und Emotionen erkennen.

Mit der körperlich ausgedrückten Involviertheit und den beschriebenen Ambivalenzen kommt zugleich auch der Erfahrungsmodus des „Dazwischenstehens“ im Sinne eines „Nebeneinander[s] von nicht zu vereinbarenden Zuständen und Situationen“ (Hentschel 2010: 238) zum Ausdruck, in dem die Schüler/innen im Kontext der Simulation oszillieren. So kann beispielsweise auch die mit Blick auf die bisherige Rollenausgestaltung für den Kirchenvertreter sprachlich eher unangemessene Äußerung „Seid doch mal ruhig jetze“ in verschiedenen Relationen interpretiert werden: Zum einen wird damit in Reaktion auf die Unterbrechungen und vorgebrachten Einwände die als leibgebundene erfahrene Emotion des Ärgers und der Ungeduld angesichts der Unterbrechungen zum Ausdruck gebracht; zum anderen verweist die Äußerung auf im Handlungsrahmen Unterricht geltende Regeln und Normen. Darüber hinaus lassen sich auch - nicht direkt mit diesem Handlungsrahmen in Beziehung stehende - ironisierende Distanzierungen beobachten, wie beispielsweise in der Äußerung „Nee, ich krieg kein Kind (mehr.)“ bis hin zu einem expliziten Verlassen der Rolle wenig später (Sm?: „Wo steht dat denn?“, Sm11: „Hier auf meinem Zettel.“, SuS: @@@(Aufzeichnungseinheit 1993: \# 00:50:34 )). Dass die Dramaturgie des Diskurses 
an solchen Stellen in gemeinsamen Gelächter konvergiert, führt zu der Vermutung, dass es sich bei diesem Modus des „Dazwischen“ in seinen verschiedenen Relationen um einen konjunktiv geteilten Erfahrungsraum handelt (Bohnsack/Przyborski 2010: 234-235) - zumal im gesamten Spielverlauf eine Reihe ähnlicher Distanzierungen der Akteure von ihren Rollen beobachtet werden können: So lässt sich beispielsweise Sm16 in der Rolle des CDU/CSU-Vertreters wiederholt zu selbstironischen Äußerungen hinreißen („Das Geld, äh, kommt aus der Staatskasse wie immer“ (Aufzeichnungseinheit 1993: \# 00:29:29); „Ja also, was das Volk glaubt, das weiß ich jetzt nicht so genau“ (ebd.: \# 00:30:11)) oder Sm4 distanziert sich mittels kleiner, relativierender Zusätze wie „,doch schon Leben“ und „im Prinzip beendet" in den Formulierungen von in der Rolle des Arztes vertretenen Inhalten (,und insofern sollte da die Frau nicht mehr entscheiden können, dass sie dieses dann doch schon Leben im Prinzip beendet“" (ebd.: \# 00:23:05)).

Wenn nun dieser Erfahrungsmodus des „Dazwischen“ im Sinne einer für politisches Lernen bedeutsamen Ambiguitätserfahrung interpretiert werden soll, erscheint es gewinnbringend, die Aufmerksamkeit auf den Schüler Sm11 zu richten, der die Position des Kirchenvertreters, die ihm - wie Äußerungen in der Vorbereitungs- und Auswertungsphase vermuten lassen (Aufzeichnungseinheit 1993: \# 00:04:25; \# 01:18:39) nicht besonders nahe zu sein scheint, äußerst engagiert vertritt. Es käme nun der Auswertungsphase zu, die mit dem performativen Vollzug dieses Perspektivwechsels verbundenen leibgebunden Erfahrungen auch diskursiv zugänglich zu machen, Dynamiken des Diskursverlaufes herauszuarbeiten und schließlich einen inhaltlichen Sachbezug zur realen politischen Debatte herzustellen. Mit Blick auf die hier exemplarisch analysierte, videographierte Unterrichtseinheit ist allerdings relativ schnell zu erkennen, dass die Auswertungsphase solchen Anforderungen nicht gerecht wird: Eine Distanzierungsphase, in der auch Emotionen im Spielverlauf thematisiert werden, ist nicht zu erkennen und insgesamt ist das Verhältnis von Durchführung und Auswertung mit 3:1 geradezu auffällig umgekehrt. Nichtsdestotrotz erlauben es abschließende Statements - wie z. B. das von Sm11: „Ich möcht auch sagen, dass man erst mal überhaupt richtig erfahren hat, was es da eigentlich, was es da eigentlich für Möglichkeiten für verschiedene Gruppen gibt undsoweiter, [...] also dass man sich eben ne richtige Meinung bilden konnte.“ (Aufzeichnungseinheit 1993: \# 01:19:25) - an der Unterstellung eines spezifischen Potenzials simulativer Unterrichtsmethoden im Politikunterricht festzuhalten. Begründet ist dieses im performativen Vollzug der sozialen Perspektivenübernahme, der es erlaubt, Prozesse politischer Urteilsbildung und Entscheidungsfindung als nicht nur an Rationalität gebundene zu erfahren und zu verstehen.

\section{Fazit und Ausblick}

Es ist nun nicht die Absicht dieses Beitrags, die nicht hinreichend erfolgte Aufarbeitung der Talkshow in der anschließenden Reflexionsphase in dieser bereits über 20 Jahre zurückliegenden Unterrichtsaufzeichnung zu kritisieren. Vielmehr soll abschließend der Ertrag dieser als konzeptionelle Vorarbeit verstandenen exemplarischen Analyse im Sinne eines Ausblicks auf anstehende Forschungsvorhaben zur Förderung von Mündigkeit im sozialwissenschaftlichen Unterricht zusammengefasst werden. Angesichts der Zweifel, 
die an einer einseitigen Orientierung am Rationalitätsprinzip in der politischen Bildung geäußert wurden, hieße die Förderung von Mündigkeit auch, darüber hinausgehende, die Prozesse politischen Urteilens, Handelns und Entscheidens leitende Faktoren im Unterricht thematisch werden zu lassen und zu reflektieren. Insbesondere in Bezug auf die Förderung der politischen Urteilsbildung im Kontext von Konflikten, die auf unterschiedliche Wertorientierungen zurückgehen, sind der rationalen Analyse und Problemlösung Grenzen gesetzt, und es gilt zunächst, die verschiedenen Dimensionen des Konfliktes überhaupt erst nachvollziehbar werden zu lassen (Breit/Reichenbach 2005: 32).

Wie mit der exemplarischen Analyse gezeigt wurde, können diese verschiedenen Dimensionen, die einer ausschließlich kognitiven Auseinandersetzung vermutlich so nicht zugänglich wären, im performativen Vollzug der Perspektivenübernahme leibgebunden erfahren werden. Der spezifischen und situativen Dynamik des Diskurses folgend werden die Begrenztheit rationaler Argumente sowie die Bedeutung emotionaler Aspekte in dem simulierten Prozess der politischen Urteilsbildung und Entscheidungsfindung von den Schüler/-innen in der wechselseitigen Verstärkung von Körper-Eindruck und -Ausdruck interaktiv und performativ hervorgebracht und im Erfahrungsmodus des „Dazwischen“ weiter bearbeitet. Wie schließlich die leibgebundenen Erfahrungen innerhalb der simulierten Auseinandersetzung im Sinne eines nicht auf Rationalitätsaspekte reduzierten Mündigkeitsbegriffes und einer damit verbundenen Ambiguitätstoleranz in der anschließenden Reflexionsphase fruchtbar aufgearbeitet werden können, wird Gegenstand zukünftiger Forschungen sein.

\section{Autorenangaben}

May Jehle

Goethe-Universität Frankfurt am Main

Fachbereich Gesellschaftswissenschaften, Institut für Politikwissenschaft

jehle@em.uni-frankfurt.de

\section{Literatur}

Aufzeichnungseinheit (1993): Talkshow § 218 (v_fu_14a). Aus: Datenkollektion (20132015): Schluß, Henning/Jehle, May: Quellensicherung und Zugänglichmachung von Videoaufzeichnungen von Unterricht der Freien Universität Berlin. In: Audiovisuelle Aufzeichnungen von Schulunterricht in der Bundesrepublik Deutschland. Forschungsdatenzentrum Bildung am DIPF, Frankfurt/Main. DOI: 10.7477/19:1:2.

Autorengruppe Fachdidaktik (2016): Was ist gute politische Bildung? Leitfaden für den sozialwissenschaftlichen Unterricht. Schwalbach/Ts.: Wochenschau-Verlag.

Bilstein, Johannes/Winzen, Matthias/Wulf, Christoph (Hrsg.) (2005): Anthropologie und Pädagogik des Spiels. Weinheim, Basel: Beltz Verlag.

Birgmayer, Renate (2011): Planspielleistungen beurteilen - ein Widerspruch? In: Hitzler, S./Zürn, B./Trautwein, F. (Hrsg.): Planspiele - Qualität und Innovation. Neue Ansätze aus Theorie und Praxis. Norderstedt: Books on Demand, S. 39-56. 
Blötz, Ulrich (Hrsg.) (2015): Planspiele und Serious Games in der beruflichen Bildung. Auswahl, Konzepte, Lernarrangements, Erfahrungen - aktueller Katalog für Planspiele und Serious Games 2015. Bielefeld: W. Bertelsmann Verlag.

Bohnsack, Ralf/Przyborski, Aglaja (2010): Diskursorganisation, Gesprächsanalyse und die Methode der Gruppendiskussion. In: Bohnsack, R./Przyborski, A. (Hrsg.): Das Gruppendiskussionsverfahren in der Forschungspraxis. Opladen, Farmington Hills: Verlag Barbara Budrich, S. 233-248.

Breidenstein, Georg/Tyagunova, Tanja (2012): Ethnomethodologie und Konversationsanalyse. In: Bauer, U./Bittlingmayer, U. H./Scherr, A. (Hrsg.): Handbuch Bildungs- und Erziehungssoziologie. Wiesbaden: Springer VS, S. 387-403.

Breit, Gotthard (1991): Fühlen und Denken im politischen Unterricht. In: Schiele, S./Schneider, H. (Hrsg.) (1991): Rationalität und Emotionalität in der politischen Bildung. Stuttgart: J. B. Metzler, S. 58-78.

Breit, Heiko/Reichenbach, Roland (2005): Emotion und demokratisches Lernen. In: Reichenbach, R./Breit, H. (Hrsg.): Skandal und politische Bildung. Aspekte zu einer Theorie des politischen Gefühls. Berlin: Logos Verlag, S. 13-42.

Engartner, Tim/Siewert, Markus B./Meßner, Maria T./Borchert, Christiane (2015): Politische Partizipation 'spielend' fördern? Charakteristika von Planspielen als didaktisch-methodische Arrangements handlungsorientierten Lernens. In: Zeitschrift für Politikwissenschaft 25, 2, S. 189-217.

Erickson, Frederick (1992): Ethnographic Microanalysis of Interaction. In: LeCompte, M./ Millroy, W. L./Preissle, J. (Hrsg.): The Handbook of Qualitative Research in Education. San Diego, London: Academic Press, S. 201-225.

Fischer-Lichte, Erika (2001): Verkörperung/Embodiment. Zum Wandel einer alten theaterwissenschaftlichen in eine neue kulturwissenschaftliche Kategorie. In: Fischer-Lichte. E./Horn, C./ Warstat, M. (Hrsg.): Verkörperung. Tübingen, Basel: A. Francke Verlag, S. 11-25.

Fischer-Lichte, Erika (2004): Ästhetik des Performativen. Frankfurt am Main: Suhrkamp.

Grammes, Tilman (1999): Handlungsorientiertes Lernen. In: Mickel, W. W. (Hrsg.): Handbuch zur politischen Bildung. Bonn: Bundeszentrale für politische Bildung, S. 212-217.

Henkenborg, Peter. (2012): Politische Urteilsfähigkeit als politische Kompetenz in der Demokratie - der Dreiklang von Analysieren, Urteilen und Handeln. In: Zeitschrift für Didaktik der Gesellschaftswissenschaften 3, 2, S. 28-50.

Hentschel, Ulrike (2010): Theaterspielen als ästhetische Bildung. Über einen Beitrag produktiven künstlerischen Gestaltens zur Selbstbildung. Berlin et al.: Schibri-Verlag.

Herrle, Matthias (2013): Mikroethnographische Interaktionsforschung. In: Friebertshäuser, B./ Seichter, S. (Hrsg.): Qualitative Forschungsmethoden in der Erziehungswissenschaft. Eine praxisorientierte Einführung. Weinheim, Basel: Beltz Juventa, S. 119-152.

Jehle, May/Blessing, Benita (2014): Using Classroom Recordings in Educational History Research. An East German Civics Lesson. In: Journal of Social Science Education 13, 1, S. 118-136.

Juchler, Ingo (2005a): Politische Urteilsbildung - Kernkompetenz für den Politikunterricht. In: Weißeno, G. (Hrsg.): Politik besser verstehen. Neue Wege der politischen Bildung. Schwalbach/Ts.: Wochenschau-Verlag, S. 62-75.

Juchler, Ingo (2005b): Demokratie und politische Urteilskraft. Überlegungen zu einer normativen Grundlegung der Politikdidaktik. Schwalbach/Ts.: Wochenschau-Verlag.

Kellermann, Ingrid (2012): Emotionen - Formen - Gesten. Ein ethnographischer Blick auf verborgene Dimensionen von Unterricht. In: Zeitschrift für Erziehungswissenschaft 15, Sonderheft 16, S. 97-114.

Massing, Peter (1998): Handlungsorientierter Politikunterricht. Ausgewählte Methoden. Schwalbach/Ts.: Wochenschau-Verlag. 
Meseth, Wolfgang/Proske, Matthias/Radtke, Frank-Olaf (2012): Kontrolliertes Laissez-faire. Auf dem Weg zu einer kontingenzgewärtigen Unterrichtstheorie. In: Zeitschrift für Pädagogik 58, 2, S. 223-241.

Oeftering, Tonio/Uhl, Herbert (2010): Emotionen und politisches Lernen. In: Lange, D./Reinhardt, V. (Hrsg.): Inhaltsfelder der politischen Bildung. Band 3. Baltmannsweiler: Schneider-Verlag Hohengehren, S. 56-70.

Reinhardt, Sybille (2005a): Moralisches Lernen. In: Sander, W. (Hrsg.) (2005): Handbuch politische Bildung. Schwalbach/Ts.: Wochenschau-Verlag, S. 363-378.

Reinhardt, Sybille (2005b): Handlungsorientierung. In: Sander, W. (Hrsg.) (2005): Handbuch politische Bildung. Schwalbach/Ts.: Wochenschau-Verlag, S. 146-155.

Schluß, Henning/Jehle, May (Hrsg.) (2013): Videodokumentation von Unterricht. Zugänge zu einer neuen Quellengattung der Unterrichtsforschung. Wiesbaden: Springer VS.

Schröder, Hendrik (2015): Eine kritische Betrachtung von Rationalität als zentraler normativer Bezugspunkt des Politischen Urteils in Hinblick auf die Praxis. In: Juchler, I. (Hrsg.): Hermeneutische Politikdidaktik. Perspektiven der politischen Ethik. Wiesbaden: Springer VS, S. 139-151.

Weber, Florian (2016): Emotion und Kognition in der Politischen Bildung. Überlegungen aus emotionstheoretischer Sicht. In: Deichmann, C./May, M. (Hrsg.): Politikunterricht verstehen und gestalten. Wiesbaden: Springer VS, S. 165-183.

Westphal, Kristin (2005): Möglichkeitsräume im theatralen Spiel und ihre Bedeutung für Sinnstiftungsprozesse. In: Bilstein, J./Winzen, M./Wulf, C. (Hrsg.) (2005): Anthropologie und Pädagogik des Spiels. Weinheim, Basel: Beltz Verlag, S. 103-122.

Wulf, Christoph (2005a): Zur Genese des Sozialen. Mimesis, Performativität, Ritual. Bielefeld: transcript Verlag.

Wulf, Christoph (2005b): Spiel. Mimesis und Imagination, Gesellschaft und Performativität. In: Bilstein, J./Winzen, M./Wulf, C. (Hrsg.) (2005): Anthropologie und Pädagogik des Spiels. Weinheim, Basel: Beltz Verlag, S. 15-22.

Wulf, Christoph/Zirfas, Jörg (2007): Performative Pädagogik und performative Bildungstheorien. Ein neuer Fokus erziehungswissenschaftlicher Forschung. In: Wulf, C./Zirfas, J. (Hrsg.): Pädagogik des Performativen. Theorien, Methoden, Perspektiven. Weinheim, Basel: Beltz Verlag, S. 7-40. 


\title{
Allgemeiner Teil
}

Alexandra Flügel

\section{Die Organisation der Arbeit am Gruppentisch - Subjektpositionen im Spannungsfeld zwischen Aufgabenstellung und Peers}

\begin{abstract}
Zusammenfassung
In diesem Beitrag wird die Platzierung von Schüler_innen im Unterricht an Gruppentischen aus einer anerkennungstheoretischen Perspektive betrachtet und danach gefragt, welche Subjektpositionen der Akteure am Gruppentisch sichtbar werden. Anhand der Rekonstruktion der Subjektpositionen über das Adressierungsgeschehen am Gruppentisch in einer videographierten Sequenz wird beispielhaft das Verhältnis von peerkulturellen und unterrichtlichen Anforderungen ausgelotet.

Schlagwörter: Gruppentisch, Anerkennungstheorie, Peer-Kultur-Forschung, Videographie
\end{abstract}

The Organisation of Work at the Group Table - Subject Positions in the Tension Field between Task and Peers

This paper takes a recognition-theoretical look at the positioning of students at group tables in class and discusses, which subject positions of the actors at the group table become visible. Using the reconstruction of subject positions with regard to the addressing at the group table in a videotaped sequence, the ratio of peer-cultural to instructional requirements is assessed. Keywords: Group Table, Recognition Theory, Peer Culture Research, Videography

\section{Einleitung}

In Grundschulen ist die Platzierung der Schüler_innen an Gruppentischen eine gängige Sitzordnungsvariante. Dies wird naheliegenderweise über Gruppenarbeit begründet und es wird argumentiert, dass durch Gruppentische „die Face-to-face-Situation innerhalb der Gruppe sehr gut vollzogen werden kann“ (Weichsel 2014: 23). Jenseits der Praxisliteratur finden sich kaum theoretische Auseinandersetzungen und empirische Befunde zum Unterricht am Gruppentisch. ${ }^{1}$ Dennoch liegt auf der Hand, dass die räumliche Organisation der Schüler_innen an Gruppentischen die Unterrichtssituation und die Bezugnahme der Schüler_innen aufeinander präfiguriert (vgl. z. B. Breidenstein 2006). Ausgehend von einer anerkennungstheoretisch inspirierten Perspektive, wie sie beispielsweise von Balzer und Ricken (2010) ausgearbeitet wurde, eröffnet sich ein spezifisches Interesse für die Mikrosituation am Gruppentisch:

„Die Tatsache, dass wir uns von anderen her selbst als ein Selbst erlernen, dass wir uns auf uns selbst zu beziehen lernen in den Kategorien und Sprachen der anderen, hintertreibt jeden Gedanken eines bloßen Nebeneinanders.“ (Ricken 2016: 51)

1 Eine Ausnahme stellt der Aufsatz von Schicke (2007) dar. 
An diese Überlegungen anknüpfend wende ich mich in diesem Artikel der Frage zu, welche Anforderungen für Schüler_innen aus der Bearbeitung von Arbeitsaufgaben am Gruppentisch erwachsen bzw. welche Subjektpositionen im Schüler_innen-Sein unter der spezifischen Platzierung im Raum an Gruppentischen unter den Bedingungen der Bearbeitung von Aufgaben bereitgestellt bzw. eingenommen werden. Eine erste Annäherung möchte ich über die Rekonstruktion einer videographierten Unterrichtssequenz versuchen. Dazu werde ich zunächst die theoretische Einbettung der Fragestellung und das methodische Vorgehen skizzieren (2). Hiernach erfolgt die Interpretation einer Sequenz hinsichtlich der Positionierungen der Akteure am Gruppentisch (3) und eine abschließende theoretische Einbettung der Interpretation in Überlegung der Peerkulturforschung im Kontext von Schule und Unterricht (4).

\section{Fragestellung und methodisches Vorgehen}

Die dem Artikel zugrunde liegende Fragestellung ist eingelassen in ein videographisches Forschungsprojekt, in dem der Frage nachgegangen wird, wie die Hervorbringung von Differenz und Gleichheit in den sozialen Praktiken der Schüler_innen geschieht, wie also ,doing bzw. undoing difference' (vgl. Hirschauer 2014) unter Schüler_innen erfolgt. Es wird danach gefragt, wie Schüler_innen unter den Bedingungen von Schule und Unterricht sich gegenseitig adressieren und welche Subjektpositionen darin zum Ausdruck kommen. Die Perspektivnahme auf Subjektpositionen bzw. das Subjektivierungsgeschehen basiert auf dem analytischen, nicht-normativen Anerkennungsbegriff in Anlehnung an Butler, wie er in der Erziehungswissenschaft beispielsweise von Balzer/Ricken (2010) und Reh/Ricken (2012) herausgearbeitet wurde. Anerkennung und Anerkennbarkeit sind an Normen ge- und mit Subjektpositionen verbunden. Der subjektkonstituierende Akt der Anerkennung ist als ambivalentes Geschehen zwischen Ermächtigung und Unterwerfung zu verstehen. Die Macht der Anerkennung verleiht Subjekten bestimmte Rechte, unterwirft sie gleichzeitig bestimmten Deutungen und legt sie auf bestimmte Eigenschaften fest. Macht ist demnach nicht jenseits des Anerkennungsgeschehens zu verorten, sondern diesem immanent (vgl. Balzer/Ricken 2010). Das Anerkennungsgeschehen findet hierbei ,nie nur zwischen zwei Personen statt, sondern stets vor dem Horizont von Normen, die erst die Kriterien dafür liefern, als wer jemand anerkannt oder eben nicht anerkannt wird“ (Fritzsche 2015: 175). Normen und Normsysteme sind allerdings nicht als vorgängige, eindeutige und stabile Systeme zu verstehen, sondern können miteinander konkurrieren und im Widerspruch zueinander stehen. Einer Rekonstruktion zugänglich werden die Normen der Anerkennung im Adressierungsgeschehen, welches in Praktiken erfolgt. Orientiert an der von Balzer und Ricken (2010) formulierten heuristischen Frage wird versucht, das Adressierungsgeschehen rekonstruierbar zu machen: „Als wer [wird; A.F] jemand von wem und vor wem wie angesprochen und adressiert [...] und zu wem [wird; A.F.] er/sie dadurch vor welchem (normativen) Horizont sprachlich bzw. materiell etablierter Geltung gemacht $[\ldots]^{\text {“ }}($ ebd.: 73$)$ ?

Im Kontext dieses Forschungsprojektes wurden fünf Grundschulklassen (3. und 4. Schuljahr) aus verschiedenen Grundschulen (Stadt, Land, verschiedene Stadtteile mit 
unterschiedlicher Sozialstruktur, Grundschulen mit inklusivem Anspruch und nicht explizit inklusiv arbeitende Schulen) videographisch (vgl. Herrle/Dinkelaker 2016) begleitet. Im Zuge einer fokussierten Ethnographie (Knoblauch 2001) wurde der Kamerafokus auf Gruppentische ausgerichtet, um das Interaktionsgeschehen unter den Schüler_innen in den Blick nehmen zu können. Bei der Auswertung des Materials hinsichtlich des gegenseitigen Adressierungsgeschehens der Schüler_innen und den darin enthaltenen Praktiken des , doing bzw. undoing difference' habe ich mich dazu entschieden, um der Gefahr der Reifizierung bewusst zu begegnen und offenzuhalten, welche Praktiken im Feld relevant werden, keine Suchstrategie orientiert an einzelnen und vorab bestimmten Differenzlinien vorzunehmen. Allerdings müssen, nicht zuletzt auf Grund der Datenfülle und der Überkomplexität von Unterricht, die sich in den videographischen Aufzeichnungen niederschlägt, Selektionsentscheidungen getroffen werden. Da der Fokus auf die gegenseitige Adressierung von Schüler_innen und die darin zum Ausdruck kommenden Subjektpositionen unter den Bedingungen von Schule und Unterricht gerichtet ist, segmentiere ich das Datenmaterial entlang der Situationskonstellationen im Unterricht, also des Bearbeitungssettings, welchem die Schüler_innen im Unterricht zu begegnen haben. Für diesen Artikel konzentriere ich mich auf die Situationskonstellation ,Einzelarbeit am Gruppentisch' und frage nach den (verschiedenen) Subjektpositionen im Schüler_innen-Sein im Fall der spezifischen Platzierung im Raum an Gruppentischen unter den Bedingungen der Einzelarbeit.

Die Auswertung des Materials kann als eine Kombination von Kodierung ${ }^{2}$ und sequenzanalytischer Rekonstruktion beschrieben werden (vgl. z.B. Breidenstein/ Hirschauer u. a. 2015). Zuerst wird die offene Kodierung jeweils separat für das spezifische Bearbeitungssetting (Einzelarbeit, Gruppenarbeit, Frontal-, Übergangsphasen) vorgenommen. Hieran schließt sich das fokussierte, systematische Kodieren an, welches zum Ziel hat, aus dem Datenmaterial eine analytische Ordnung zum Vorschein zu bringen (vgl. ebd.: 135f.). Auf der Basis dieser Kodierung werden einzelne Sequenzen, die fragestellungsgeleitet für die jeweilige Kategorie als besonders dicht und exemplarisch gewertet werden (zu den Kriterien vgl. ebd. 140ff.), einer sequenzanalytisch, rekonstruktiven Feinanalyse unterzogen (vgl. Deppermann 2008). Der Wechsel zwischen systematischem Kodieren und sequenzanalytischer Rekonstruktion ist als iterativer Prozess zu verstehen. Die ausgewählten Sequenzen werden verbal transkribiert und ebenso hinsichtlich des im Video visuell Wahrnehmbaren sequenziell interpretiert (vgl. ebd.). Das jenseits der Tonspur Stattfindende (Mimik/Gestik, Körper-Raum-Konstellationen, Artefakte) wird dabei, in Anlehnung an Reh (2012), ebenfalls verschriftlicht und festgehalten, jedoch bleibt die Videoaufnahme das zentrale Datum der Interpretation: „Das schriftliche Erfassen der abgebildeten Abläufe dient als Protokoll eines genauen Hinsehens“" (Reh 2012: 163). In einer szenischen Beschreibung wird dann Gesagtes und

2 Orientiert an dem sensibilisierenden Konzept der Anerkennung und den sich darin zeigenden Subjektpositionen werden die Daten orientiert am Kodierverfahren der Grounded Theory (Strauss/Corbin 2010) analytisch aufgebrochen. Allerdings wurde das Kodierverfahren angesichts der praxistheoretischen Reformulierungen der Grounded Theory dahingehend angepasst, so dass nicht mehr dem handlungstheoretischen Kodierparadigma gefolgt wird und so nicht mehr die Teilnehmer_innen im Vordergrund stehen, sondern Situationen (vgl. Strübing 2014: 100ff.). 
Gesehenes einer Sequenz „möglichst präzise als ,verstandene [s; A.F.]' notiert“ (ebd.), was bedeutet, dass der Beschreibung ,die Rekonstruktion des körperlichen Tuns und der sprachlichen Äußerungen [...] in ihrem spezifischen räumlich-zeitlichen Kontext“ (ebd.: 163f.) inhärent ist und diese den sequenziellen Sinnaufbau abbildet. Insofern handelt es sich bei der szenischen Beschreibung immer auch um ein bereits, verstandenes ' und gedeutetes Produkt, welches verdichtete Beschreibungen und somit Kürzungen in der Beschreibung des Gesehenen und Gesagten mit sich bringt.

\section{Subjektpositionen am Gruppentisch}

Im Folgenden wird eine Sequenz dargestellt und interpretiert, die der aus dem Material herausgearbeiteten Kategorie der, Gleichzeitigkeit von schulischer Bearbeitung und peerkultureller Aktivität‘ zugeordnet wurde. Das anonymisierte Standbild, welches in die szenische Beschreibung der Sequenz integriert wurde, dient an dieser Stelle lediglich der Orientierung für den/die Leser_in.

\section{1 „Da hab’ ich ja noch mal Glück gehabt, dass ich da heile durchgekommen bin mit der Nummer" - Szenische Beschreibung}

Im Deutschunterricht einer vierten Klasse gibt die Lehrerin die Aufgabe, dass jede_r Schüler_in vier Sätze mit wörtlicher Rede formulieren und notieren soll. Die Lehrerin, Frau Hinterland, beendet ihre Aufgabenstellung mit den Worten: „Es gibt Smileys für die Kinder, die fleißig arbeiten. Stillezeit beginnt.“ Drei der videographierten Schüler_innen am Gruppentisch blicken in ihre Hefte, Timo (rechts hinten) blickt umher und dreht seinen Stift mit den Fingern, berührt dann seinen Sitznachbarn Dustin (rechts vorne) an der Schulter und sagt: ,Schreib doch: ,Dustina denkt.““ Dabei blickt er erst lächelnd zu Dustin und schaut dann zu dem ihm schräg gegenübersitzenden Jonas (links vorne), der auch kurz lächelt und sofort formuliert: „A Astrid denkt.“ Dabei blickt Jonas im Klassenraum umher, öffnet weit die Augen und nickt (als wäre ihm eine Idee gekommen). Er beugt sich über sein Heft und beginnt zu schreiben. Timo: „Was ist? Was ist, Jonas?“ Dieser reagiert nicht. Timo: „Jonas, was ist?“ Kichernd blickt Jonas weiterhin auf sein Heft. 


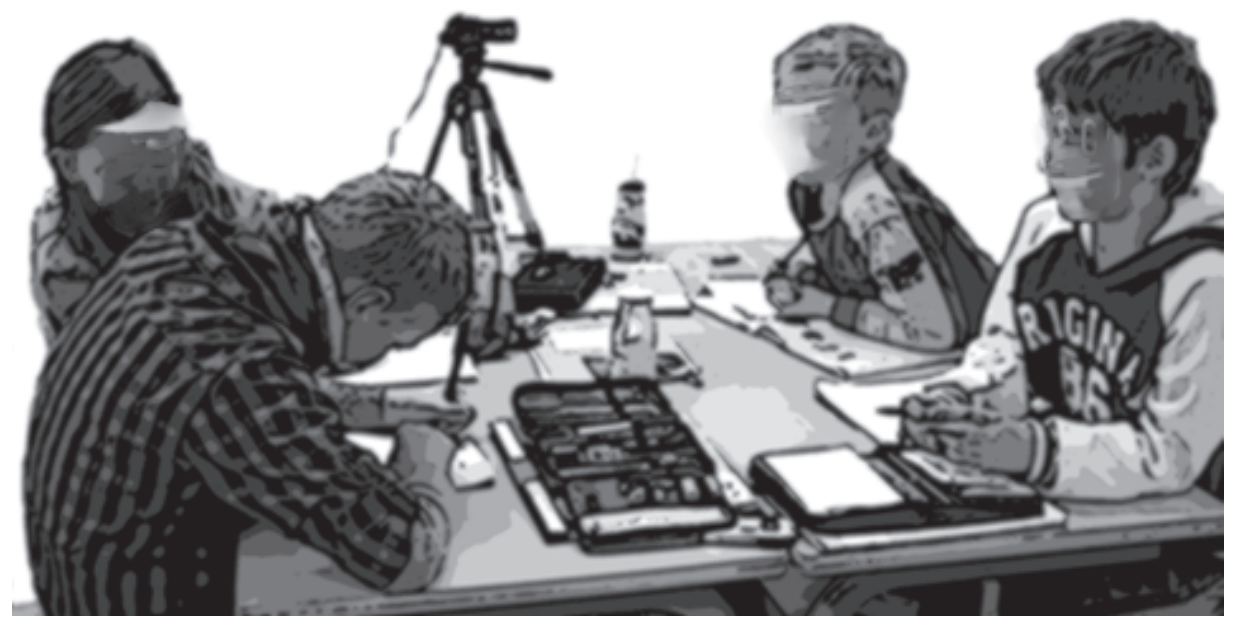

Abb. 1: Standbild zur beschriebenen Sequenz

Die drei anderen am Tisch beugen sich zu Jonas und versuchen in sein Heft zu blicken. Eine Schülerin vom Nachbartisch mutmaßt: „Der weint“, was Dustin verneint. Jonas blickt auf, schaut umher und lacht. Erneut fragt Timo: „Was steht da? Was steht da?“ Jonas liest vor: „Ingeborg ruft“, und lacht hinter den vor den Mund gehaltenen Händen. Timo versucht über den Tisch gebeugt weiter in Jonas` Heft zu lesen. Jonas liest weiter vor: „Ingeborg ruft: ,Scheiße, die Eier!'“ Timo, Angeline und Jonas lächeln und lehnen sich auf ihren Stühlen zurück. Dustin blickt zu Angeline und lehnt sich ebenfalls auf seinem Stuhl zurück. Timo, Jonas und Angeline schreiben, Dustin bewegt, während er auf sein Heft blickt, die Stifthülle mit seinen Händen. Timo hebt sein Heft nach oben und wendet es Jonas zu: „Guck mal, was ich geschrieben habe.“ Jonas lächelt. Timo lächelt. Dustin sucht den Blickkontakt zu Timo, welcher nicht erwidert wird. Dustin blickt noch einige Zeit lang auf Timos Heft, während die anderen drei wieder in ihre Hefte schreiben, und wendet sich dann seinem Heft zu und schreibt. Leicht schnaubend formuliert Timo: „Karl sagt.“ Er sucht wiederum Blickkontakt zu Jonas, der diesen allerdings nicht erwidert, sondern auf sein Heft blickt und schreibt. Dustin hingegen blickt sofort auf und sucht Blickkontakt zu Timo, der diesen nicht erwidert. Alle am Tisch Sitzenden schauen wieder auf ihre Hefte herab und schreiben. Jonas fängt nach einiger Zeit an zu sprechen. Sofort unterbricht Dustin sein Schreiben und wendet seine Aufmerksamkeit ihm zu. Dieser formuliert ohne aufzublicken: „Weißt du, was ich geschrieben habe? Spacko labert: ,Ich kaufe eine Zeitung. '“ Er blickt auf zu Timo und lächelt dann in die Runde. Timo erwidert sein Lächeln und sagt: „Was?“ Jonas wiederholt das bereits Vorgelesene und alle Schüler_innen am Gruppentisch blicken zu Jonas und lächeln. Dustins Blicke wandern zwischen Jonas und Timo hin und her. Timo sagt leicht ermahnend Jonas' Namen, lächelt und blickt zur Videographin, auch Jonas wendet sich zu ihr um. Die Lehrerin, die mit dem Rücken zum Gruppentisch steht, dreht sich um und blickt, ohne 
etwas zu sagen, in die Runde. Jonas, Timo und Angeline blicken wieder auf ihre Hefte und beginnen zu schreiben. Dustin schaut umher und spielt mit der Hülle seines Stiftes.

Timo lacht leise auf und sagt: „Carlos sagt:,Meine Güte, is dat geil““, worauf Dustin zu Timo schaut. Dieser blickt Jonas an und lacht. In diesem Moment beugt sich Dustin zu seinem Heft hinunter und schreibt. Nachdem eine Zeit lang alle vier in ihre Hefte geschrieben haben, Dustin immer wieder auch umhergeblickt, mit der Stifthülle gespielt und seinen Kopf mit dem Gesicht zur Tafel auf den Tisch gelegt hat, schiebt Jonas sein Heft unter sein Etui, schaut zu Timo hinüber und sagt, dass er fertig sei. Timo fragt nach Jonas' Sätzen und dieser holt sein Heft wieder hervor und liest die Sätze vor. Timo und Dustin blicken Jonas beim Vorlesen an: „Ich habe geschrieben: Astrid denkt: ,Habe ich Deutsch?' Ingeborg ruft: ,Scheiße, die Eier!' Spacko ...“ Jonas und Timo fangen an zu lachen, Dustin lächelt und auch Angeline richtet sich nun auf und blickt zu Jonas: „... labert: ,Ich kaufe eine Zeitung' Jonas fragt: ,Habe ich heute Schule?'“ Jonas senkt seinen Kopf und schaut wieder auf sein Heft und sofort beginnt Timo, seinen Blick immer wieder zu Jonas gewandt, aus seinem Heft vorzulesen: „Carlos sagt: ,Meine Güte, is dat geil!"“ „Was?“, fragt Jonas, während er sein Heft wieder unter sein Etui schiebt und Timo wiederholt seinen Satz. Frau Hinterland kommt zum Gruppentisch und zieht Jonas Heft unter seinem Etui hervor. Im Flüsterton sagt sie: „Zeig mir mal!“ Angeline und Timo lachen, während die Lehrerin ins Heft blickt. Jonas hält seine Hände vor sein Gesicht. Dustin blickt zu Jonas und spielt mit seinem Stift. Die Lehrerin sagt lächelnd: „Ingeborg.“ Jonas darauf: „Ja, so heißt unsere Nachbarin mit Vornamen.“ Darauf Timo: „Echt?“ Die Lehrerin wendet ihr Gesicht in Richtung anderer Gruppentische und macht ein „Pscht“-Geräusch. Sie reicht Jonas das Heft und sagt: „Ja. Gut“, wendet sich ab und geht vom Tisch weg. An die Klasse gewandt formuliert sie: „So, hier ist Stillezeit.“ Angeline und Timo blicken wieder auf ihre Hefte und schreiben. Dustin blickt in Richtung Jonas und führt seinen Stift an den Mund. Nur für die Tischgruppe hörbar sagt Jonas: „Da hab ich ja noch mal Glück gehabt, dass ich da heile durchgekommen bin mit der Nummer.“ [...] Frau Hinterland, mit dem Rücken zum videographierten Tisch stehend, ruft in die Klasse: „So, Edem und Vanessa haben einen Heuli!“”

\subsection{Interpretation der Sequenz}

Die Lehrerin rahmt die Situation als Leistungssituation, in der die Schüler_innen als solche adressiert werden, die eine bestimmte Arbeitshaltung (fleißig sein) einzunehmen haben. Die in Aussicht gestellte Honorierung bezieht sich auf die Arbeitshaltung und nicht auf die Qualität eines Arbeitsproduktes oder -ergebnisses. Potentiell wird allen Schüler_innen die Zugänglichkeit zur Belohnung (Smileys) suggeriert, obwohl ebenso angedeutet wird, dass nicht alle Schüler_innen diese erhalten werden. Die erwartete Arbeitshaltung wird an Lautstärke gebunden: Stillezeit! Im akustischen Raum und der Öffentlichkeit des Gruppentisches findet eine spielerisch-humoristisch aufgeladene Bearbeitung des Arbeitsauftrages statt, die bei allen Schüler_innen am Gruppentisch zu sichtbarer Freude und Spaß führt. Jedoch sind unterschiedliche Positionen zu beobachten: Produzenten der humorvollen Bearbeitung sind Timo und Jonas, wobei Jonas seine witzige Idee mimisch und gestisch pointiert in Szene setzt. In der, vermeintlichen Geheimnisproduktion`, an der sich alle beteiligen, wird die Bedeutsamkeit dessen, 
was Jonas in sein Heft notiert, kollektiv hervorgebracht. Dustin und Angeline zeigen sich als Interessierte, Zuhörende und Aufmerksamkeit-Schenkende. Timo, der zu Beginn die Spielidee eingeführt hat, bereitet Jonas durch sein beharrliches Nachfragen den Aufmerksamkeitsraum für das In-Szene-Setzen seiner Satzkonstruktion, die eine schulische Aufgabe zu einem Moment des peerbezogenen Amüsements modelliert. Jonas wird adressiert als einer, der etwas Geheimnisvolles und Bedeutsames produziert hat. Dass diese Inszenierung über den Gruppentisch hinweg Beachtung findet, markiert die Schülerin, die sich an der Deutung von Jonas“ Verhalten beteiligt („Der weint.“). Das gemeinsame Amüsement wahrt den unterrichtlichen Rahmen (stille und fleißige Bearbeitung einer Einzelaufgabe) und wird weder von der Lehrerin noch von anderen Schüler_innen als unpassend markiert. Dass die unterrichtliche Rahmung ohnehin dominant bleibt, zeigt sich darin, dass alle Beteiligten nach der ,Pointe“ wieder in ihre körperlich demonstrierte und sichtbare Arbeitshaltung zurückkehren.

Im weiteren Verlauf der Sequenz sind wieder Jonas und Timo diejenigen, die sprechend agieren und sich durch die Blickrichtung und die Wahl der Ansprache gegenseitig als Ansprechpartner adressieren (Timo zu Jonas: „Guck mal, was ich geschrieben habe."). Timo adressiert Jonas exklusiv, aber tischöffentlich und präsentiert seine Arbeitsleistung. Dustins Bemühungen, auch in das Heft zu schauen, werden nicht berücksichtigt. Das in der Öffentlichkeit des Gruppentisches stattfindende Zwiegespräch zwischen Jonas und Timo kreist um die gegenseitige Aufmerksamkeit. Entscheidend dabei scheint jedoch zu sein, ob von Jonas Beachtung geschenkt wird. Timos Sprachhandlung bleibt unvollendet, da sie von Jonas nicht mit Aufmerksamkeit belegt wird. Während Timos Blicke fortwährend evaluierend zu Jonas wandern, spricht dieser hingegen ohne aufzublicken in die Runde. Er versichert sich nicht, ob sein Gesagtes auf Interesse bei den anderen stößt, sehr wohl aber, welche Wirkung dieses erzeugt. Dustin gelingt es nicht auf seine Kontakt suchenden Blicke Erwiderung zu erfahren. Jonas' zweite Satzkonstruktion erfährt wiederum kollektive Beachtung und erregt Amüsement. Der Blick zur Videographin, nachdem Jonas ein Schimpfwort in seine Formulierung eingebaut hat, deutet darauf hin, dass ausgelotet wird, ob im Grenzgang zwischen Bearbeitung und Konvertierung eine Überschreitung stattgefunden hat bzw. ob im Verhalten der Videographin eine Duldung markiert wird. Hieran wird deutlich, dass die Tischöffentlichkeit nicht als privater Raum gesehen wird, sondern allgegenwärtige Sichtbarkeit und Hörbarkeit mitschwingt. Dieses Risiko wird durch den ermahnenden Blick der Lehrerin, die sich am Nachbartisch im Gespräch befindet, bestätigt. Die wortlose Erinnerung der Lehrerin an eine unausgesprochene Norm (es ist hier nicht eindeutig herauszuarbeiten, ob es sich um den Hinweis auf den Arbeitsmodus „Stillezeit“" oder den Verweis auf die soziale Norm, keine Schimpfworte zu benutzen, handelt) überführt Jonas, Angeline und Timo sofort in ihre Arbeit dokumentierende Körperhaltung. Dustin allerdings findet nicht umstandslos in die Bearbeitung seiner Einzelaufgabe zurück und verbleibt in selbstbezüglichen Aktivitäten.

Im Austausch zwischen Timo und Jonas über ihre jeweiligen Satzkonstruktionen entsteht das Bild einer spielerischen, freundschaftlichen Bezugnahme aufeinander, die sich in Exklusivität präsentiert. Dustins Versuche der Kontaktaufnahme (Suche nach Blickkontakt, Zuschauen, körperliche Hinwendung zu Jonas und Timo) führen nicht zu einer Erwiderung. Vielmehr wird er in dieser Sequenz als Nicht-Gemeinter positioniert. 
Die Lehrerin überführt die zuvor in der Halböffentlichkeit des Gruppentisches präsentierte Aufgabenbearbeitung von Jonas in die weiterhin halböffentliche, nun aber offizielle Begutachtungs- und Bewertungssituation. Die Aufgabenmodellierung von Jonas wird von den am Tisch Sitzenden kollektiv als spielerisch-humoristische Bearbeitung mit der Option zur Riskanz re-inszeniert (lachen, Hände vor das Gesicht halten). Das Lachen der am Tisch Sitzenden bricht die Inszenierung jedoch dahingehend, dass die Situation nicht von wirklicher Sorge bestimmt zu sein scheint, was eher auf eine spielerische Aufführung von Riskanz verweist. Frau Hinterland schließt sich der humorvollen Deutung der Bearbeitung der Aufgabe an, indem sie eine inhaltlich ,unverfängliche“ Stelle (,Ingeborg“) sprachlich und lachend markiert, während die Begriffe „Scheiße“ und „Spacko“ de-thematisiert bleiben. Jonas“ Arbeitsprodukt wird von der Lehrerin, positiv evaluiert (,Ja. Gut.“) und als unterrichtsadäquat markiert. Anderen bleibt dies hingegen verwehrt, was sich in der Vergabe von Sanktionen (Heulis) dokumentiert. Indem Jonas - in der Hörweite seiner Mitschüler_innen - auf „Glück“" verweist, das ihn vor weiteren Konsequenzen bewahrt habe, wird die spielerisch-humoristische Bearbeitung seiner Aufgabe wieder in den Vordergrund gerückt, die von Beachtung durch die Peers begleitet war und somit peerkulturelle Bedeutsamkeit hat.

\subsection{Subjektpositionen - Zwischen Gemeinschaft und Nicht-Gemeint- Sein}

Positionen werden zugewiesen, zurückgewiesen, von Akteuren eingenommen oder es wird ihnen entsprochen, sie sind aber keineswegs auf eine statische Positionierung im Feld zu reduzieren: „Subjektpositionen sind [...] in einen Raum eingebunden, der durch die Subjekte erst hervorgebracht wird und dabei spezifische Möglichkeiten transportiert“ (Schütz 2015: 81). Bezogen auf die hier eingenommene Forschungsperspektive bedeutet dies, dass der Raum ,Gruppentisch in der Schule' einen Möglichkeitsraum für verschiedene Subjektpositionen darstellt, in die Akteure unterschiedlich über Adressierungen verwiesen werden. In diesen Positionierungen re-positionieren sie sich different über Re-Adressierung (vgl. Ricken 2015: 143). Die Bezugnahmen der Akteure aufeinander (körperlich und verbal) werden hinsichtlich der ,emergierenden Situationsdeutungen, normativen Orientierungen und einander zugewiesenen Positionen“" (Rabenstein/Steinwand 2016: 255) rekonstruiert. Bereits Bennewitz und Meier (2010) haben eindrücklich herausgearbeitet, dass Unterricht nicht eine Situation ist, „die von allen Schülern in gleicher Weise getragen und erfahren wird, sondern dass sie sich aus mehreren kleinen Welten zusammensetzt“" (ebd.: 101). Im Folgenden werden die in der Sequenz simultan ${ }^{3}$ sich zeigenden und miteinander interferierenden Subjektpositionen kontrastierend herausgearbeitet, um so den Möglichkeitsraum ,Gruppentisch“ hinsichtlich des Verhältnisses von Peerkultur und unterrichtlichen Anforderungen näher bestimmen zu können.

3 Bedeutung generiert sich nicht nur daraus, „dass etwas gesagt [oder getan; A.F.] wird und etwas anderes nicht, sondern auch dadurch, dass [es; A.F.] sich parallel zu anderem ereignet, was nicht diese Mitteilung, aber dennoch wahrnehmbar ist“" (Dinkelaker/Herrle 2009: 49). 
In der oben dargestellten Sequenz findet am Gruppentisch eine gemeinsame Belustigung und die Teilhabe an der humorvollen Bearbeitung einer Arbeitsaufgabe statt, in der das Amüsement unter anderem darin liegt, die Grenzen des im Unterricht Sagbaren im halböffentlichen akustischen Raum des Gruppentisches zu ,bespielen“. Die Aufführung der spielerischen Bearbeitung und gemeinsamen Belustigung ist jedoch auf die Öffentlichkeit der Gruppe angewiesen, die sich so situativ als Peer-Gruppe etabliert. Hier erfolgt nun eine Aufführung von Handlungsfähigkeit im Spielraum zwischen Peerorientierung und Unterrichtsorientierung, den der Unterricht bietet. Handlungsfähigkeit, im Sinne eines praxeologischen Zugriffs auf Agency, ist nicht zu verstehen als immer schon bestehende Eigenschaft eines autonom handelnden Akteurs, sondern wird von Kindern „in und durch Adressierungspraktiken situiert. Kinder statten sich demnach durch ihre wechselseitige Adressierung mit Handlungsmächtigkeit aus“ (Eckermann/Heinzel 2015: 35). Die verschiedenen Positionierungen der Akteure finden ihren Ausdruck über „Ermöglichung und Verhinderung von Tätigkeiten“ (Fritzsche/Idel/Rabenstein 2011: 32f.), hergestellt über die Adressierungen zum Beispiel in didaktischen Arrangements (z. B. Einzelarbeit), durch Schüler_innen und Lehrer_innen, durch die Anordnung des Mobiliars (z. B. Gruppentische) und anderer Artefakte. Mit Blick auf die Akteure in der beschriebenen Sequenz und die herauszuarbeitenden Subjektpositionen werden unterschiedliche Möglichkeitsräume von Agency erkennbar.

Betrachtet man Jonas ${ }^{4}$, wird sichtbar, dass eine Unterscheidung von Schülerpraktiken, d. h. unterrichtsnahe Praktiken, und peerkulturelle Praktiken kaum möglich ist, da ,jedes Handeln vor dem Hintergrund beider analytisch getrennter Anforderungsbereiche interpretiert werden kann“ (Bennewitz 2009: 68). Jonas kann sich einerseits als handlungsfähig hinsichtlich peerrelevanter Themen zeigen und wird von den am Tisch Sitzenden als ein solcher adressiert. Seine Äußerungen werden als relevant eingestuft, ihnen wird Beachtung geschenkt und die anderen beteiligen sich an der Inszenierung seiner humorvollen Bearbeitung. Die Nischen des Schüler innen-Seins werden also genutzt für eine kreative, humorvolle, ein wenig ,aufmüpfige' Bearbeitung. Andererseits passt sich diese mit positiver Resonanz belegte Aufführung reibungslos in die Bearbeitung der Aufgabe und die schulischen Ansprüche ein. Jonas kann sich vor der Lehrerin und auch vor seinen Mitschüler innen als ein Könnender hinsichtlich der formalen Bearbeitung der Arbeitsaufgaben präsentieren, als ein schnell Arbeitender, denn er ist als erster am Gruppentisch fertig, und als ein solcher, dessen Bearbeitung von der Lehrerin beachtet, begutachtet und gelobt wird („Ja. Gut.“). Die Lehrerin unterwirft ihn in der Adressierung als „Gut"-Leistender der Anforderungs- und Bewertungslogik des Unterrichts. Jedoch erfolgt durch Jonas ' Verweis auf die spielerisch humoristische Bearbeitung seiner Aufgabe (,Da hab ich ja noch mal Glück gehabt, dass ich da heile durchgekommen bin mit der Nummer.“) eine Re-Positionierung vor der Öffentlichkeit seiner Peers am Gruppentisch.

Während die Fokussierung auf Jonas, dessen Handlungsfähigkeit in das Zentrum rückt, sowohl hinsichtlich der Darstellung von Freundschaftsbeziehung (zu Timo), Achtung durch die Peers, aber auch hinsichtlich der unterrichtlichen Anforderungen

4 Auf Grund des begrenzten Umfangs des Artikels ist auf die Explizierung der Subjektposition von Timo verzichtet worden, da die Kontrastierung zwischen Angeline und Dustin vor der Folie von Jonas im Vordergrund steht. 
(sich arbeitend präsentieren; Arbeiten (schnell) fertig stellen; sich unterrichtsadäquat inszenieren), möchte ich nun Dustin und Angeline genauer betrachten, um ihre beiden Positionen kontrastierend gegenüberzustellen. Beide, sowohl Angeline als auch Dustin, haben bei der Aufführung der humoristischen Bearbeitung der Unterrichtsaufgabe die Position des interessierten, neugierigen, aber möglicherweise austauschbaren Publikums inne. Hierdurch sind sie als Beteiligte der sich situativ herstellenden Peer-Gruppen-Vergemeinschaftung für diese konstitutiv. Hinsichtlich der dyadischen Kommunikation zwischen Timo und Jonas werden sie jedoch zu den Nicht-Gemeinten einer exklusiven Beziehung.

Betrachtet man die Blickwechsel und Blickrichtungen in der videographischen Aufnahme genauer, wird deutlich, dass Angeline sich den am Tisch Sprechenden hin und wieder zuwendet, lacht, mit am Amüsement beteiligt ist. Sie blickt, wenn sie von ihrem Heft hochschaut, zu demjenigen, der spricht: Sie ist Beteiligte und Interessierte an der gruppenbildenden Praktik des gemeinsamen Amüsements. Jedoch wendet sie ihre Blicke nicht sofort von ihrem Heft ab, sobald Jonas oder Timo kommunizieren. Vielmehr kann eine partielle Involvierung aus Angelines Aufmerksamkeitsfoki abgeleitet werden. Beispielsweise blickt Angeline auf ihr Heft und schreibt, während Jonas und Timo in gegenseitiger Ansprache dyadisch kommunizieren. Erst als ein Schimpfwort fällt bzw. das Lachen ansteigt, richtet sie ihren Kopf auf und dreht sich zum kommunikativen Geschehen am Gruppentisch. Anders als Dustin wirkt Angeline nicht darum bemüht, Teil der Bezogenheit von Timo und Jonas sein zu wollen. So wird zwar die Position als die Nicht-Gemeinte in der freundschaftlichen und exklusiven Bezogenheit zwischen Timo und Jonas hergestellt, aber gleichzeitig macht Angeline „qua Re-Adressierung sich selbst zu jemandem“ (Ricken 2015: 143), der auch nicht danach sucht, Zugang zu dieser Exklusivität zu erlangen. Parallel dazu kann sie sich in dieser Sequenz auch als eine zeigen, die kontinuierlich zu der körperlichen Arbeitshaltung zurückkehrt und ebenso stilles und fleißiges Arbeiten präsentieren kann.

Ausgehend von der detaillierten Analyse der Blickwechsel und Blickrichtungen fällt kontrastierend hierzu bei Dustin auf, dass dieser meist zu Timo und Jonas schaut. Seine Blicke, aber auch seine körperliche Hinwendung zu den Heften von Jonas und Timo wirken so, als suche er nach Kontakt und versuche eine Position in der freundschaftlichen Bezugnahme zwischen Jonas und Timo zu etablieren, die ihm allerdings verwehrt bleibt (Seine Blicke werden nicht erwidert, Timo präsentiert ihm sein Heft nicht). Die Aufführung von freundschaftlicher und exklusiver Bezogenheit zwischen Timo und Jonas adressieren Dustin in dieser Sequenz als Ausgeschlossenen. Während Dustin im Kontext der Vergemeinschaftung am Gruppentisch über das gemeinsame Amüsement eine legitime Position des Dazugehörigen erlangt und darin gleichsam die Vergemeinschaftung mit herstellt - Unterwerfung und Ermächtigung -, bleibt es ihm verwehrt, der Gemeinte und Dazugehörige hinsichtlich der Aufführung einer freundschaftlichen Bezogenheit zu sein.

Im Gegensatz zu Angeline, die auch durch die Exklusivität zwischen Jonas und Timo die Position der Nicht-Gemeinten erhält, sich jedoch re-positionieren kann als eine, die auch nicht danach sucht, erhält Dustin in dieser Sequenz die Position des Ausgeschlossenen. Prekär wird Dustins Situation dadurch, dass er dieser Positionierung nicht durch ein ,Aus-der-Situation-Gehen` entfliehen kann. So sind seine Bewegungs- 
optionen und damit auch seine Handlungsfähigkeit durch das unterrichtliche Setting und die damit verbundenen Reglementierungen eingeschränkt. Ein Ausweichen auf andere Mitschüler_innen oder das Verlassen der Situation sind im schulischen Ordnungsrahmen nicht vorgesehen. So trägt die bestehende Sitzordnung übersituativ zur Stabilität von Positionierungen bei (vgl. Budde 2015). Am Gruppentisch sind Personen füreinander wahrnehmbar, andere sind jenseits der unmittelbaren Sichtbarkeit, da sie sich nicht im Blickfeld befinden, aber möglicherweise dennoch im akustischen Nahbereich verbleiben. Die Positionierung am Gruppentisch schafft räumliche Bedingungen für Interaktionen, Zonen der gesteigerten Sichtbarkeit und Exponierung, der Bezogenheit und Exklusivität, Räume, die die Möglichkeit zu ,privater' Kommunikation bieten, die körperliche Zugriffe zulassen und die Körper aufeinander ausrichten (vgl. Hnilica 2010).

Auch hinsichtlich der unterrichtsrelevanten Perspektive wird eine Differenz zwischen Angeline und Dustin sichtbar. Während Angelines Körper immer wieder in eine Arbeitshaltung überführt wird, zeigt sich dies bei Dustin im Verlauf der Sequenz immer weniger. Zunehmend wird er sichtbar als ein Schüler, der sich nicht als Arbeitender zeigen kann, sondern der als einer wahrnehmbar wird, der - scheinbar selbstvergessen - selbstbezogenen Aktivitäten nachgeht (schaut umher, spielt mit seiner Stifthülle, legt seinen Kopf auf den Tisch), während die anderen am Tisch auf ihre Hefte blicken und schreiben. Die Aufgabe, die die Schüler_innen dieser Klasse - und somit dieses Gruppentisches - zu bewältigen haben, ist als Einzelaufgabe formuliert und somit von dem Einzelnen zu bearbeiten und zu verantworten. In der Mikroperspektive wird jedoch deutlich, dass die Arbeitsprozesse nicht unabhängig von den Anderen gestaltet werden (vgl. Breidenstein 2006: 175). Die haptische, akustische und visuelle Nähe am Gruppentisch kann auch zu einer gewissen Schutzlosigkeit gegenüber den Handlungen der Anderen führen, die sich auf die Bearbeitungsprozesse der Einzelnen auswirken, die wiederum unter die Begutachtung schulischer Bewertungsnormen fallen.

\section{Zur Ordnung am Gruppentisch - Ausblick}

Die in der Interpretation des Geschehens am Gruppentisch sichtbar gewordene Gleichzeitigkeit, Verschränkung und Verwobenheit von peerorientierten und unterrichtsorientierten Aktivitäten ist für die Unterrichtsforschung nicht neu (vgl. z. B. Bennewitz/Maier 2010; de Boer 2009): „lehrseitige und peerkulturelle Anforderungen“ (Bennewitz/Meier 2010: 109) verzahnen sich ineinander. Die Schüler_innen stehen einerseits vor dem Auftrag, eine Einzelaufgabe alleine zu bearbeiten und diese Bearbeitung, als strukturelles Merkmal von Einzelarbeit, alleine zu verantworten. Die Platzierung am Gruppentisch verweist die Schüler_innen andererseits - und dies zeigt sich nicht nur in dieser Sequenz, sondern ist charakteristisch für das gesamte videographierte Material - in einer spezifischen Weise aufeinander: Kommunikation und Bezugnahme aufeinander scheint konstitutives Moment des Gruppentisches zu sein. Die Gestaltung der gegenseitigen Bezugnahme und die Kommunikationspflege stellt die Schüler_innen allerdings vor die Herausforderung, die immer wiederkehrende, dauerhafte Kommunikationsaufnahme, Erwiderung und auch Ablehnung mit der Erledigung der unterrichtlichen Aufgaben in Einklang zu bringen und auszutarieren. Das soziale Prinzip der Kommunikationspflege folgt dabei nicht nur den 
Ansprüchen, die sich aus den Logiken der Peer-Kultur ergeben, also Freundschaft, Sympathie und Antipathie, Zugehörigkeit und Abgrenzung, Imagepflege etc. auszuhandeln (vgl. Bennewitz/Breidenstein/Meier 2015: 289), sondern positioniert die Kinder als Schüler_innen am Tisch im Unterricht zueinander: Kommunikationspflege ist auch eine angemessene Reaktion auf die gestellte Anforderung im Unterricht, als didaktisches Setting am Gruppentisch platziert zu sein, und gehört damit zum schulischen Code. ${ }^{5}$

Demnach verweist die Interpretation darauf, dass der Unterricht in dieser Sequenz weder einfach von peerkulturellen Prozessen überformt wird (vgl. de Boer 2009: 105), noch dass diese, quasi als Hinterbühne, heimlich einen Nebenstrang zum Unterrichtsgeschehen bilden. Vielmehr treten das „Ineinander“ und die „untrennbare Aufeinanderbezogenheit" (Schütz 2015: 222) hervor. In den Interferenzen zwischen peerkulturellem und unterrichtlichem Agieren (vgl. Hertel/Pfaff 2015: 270) werden verschiedene Subjektpositionen realisiert und je spezifische Formen der Agency interaktiv hergestellt (vgl. auch Bollig/Kelle 2013: 275). Während Jonas, Timo und Angeline sich positionieren bzw. positioniert werden als Beherrscher_innen des Wechsels zwischen ,spielerische[r] Umfunktionierung von Unterrichtsinhalten“" (Breidenstein 2006: 193) und Anpassung an die Verhaltenserwartung im Unterricht (still und leise arbeiten), erfährt Dustin keine Anerkennung als ,Gemeinter' und ,Adressierter' in einer freundschaftlichen Inszenierung, der er sich, auf Grund des unterrichtlichen Settings, nicht entziehen kann. Parallel kann er sich situativ nicht mehr gemäß den Anforderungen des Unterrichts als Arbeitender zeigen. Die Bezugnahme und Herstellung von Differenz in den peerkulturellen Aufführungen wurde bislang hinsichtlich von Sympathie (Antipathie), Freundschaft (z. B. Bennewitz 2004) oder hinsichtlich der ,schulischen Leistungserbringung“ (Rose/Gerkmann 2015: 205) herausgearbeitet. In dieser Sequenz tritt jedoch darüber hinaus hervor, wie die Positionierung innerhalb der Mitschüler_innen in den peerbezogenen Aktivitäten mit der Handlungsfähigkeit und den Positionierungen im Rahmen der unterrichtlichen Codes verwoben ist. Die Betrachtung der Mikroperspektive, in der das Geschehen am Gruppentisch fokussiert wird, bietet das Potential, die über (Re-)Adressierungen und (Re-)Positionierungen hergestellten Differenz- und Hierarchieverhältnisse zwischen den Schüler_innen hinsichtlich ihres Zusammenhangs und ihrer Verwobenheit mit der unterrichtlichen Ordnung bzw. ihrem ,institutionellen Unterbau[s]“" (Eckermann/Heinzel 2015: 36) zu untersuchen. Denn es ist davon auszugehen, dass in den Adressierungen immanente Strukturen der pädagogischen Institution prozessiert und zur Aufführung gebracht werden, so wie in der hier interpretierten Sequenz die Anforderungen aus der Platzierung am Gruppentisch (Kommunikationspflege und Bezugnahme aufeinander) und der Bearbeitung einer Einzelaufgabe zu differenten Subjektpositionen (sich Leistungsfähig-zeigen-Können, Dazu-Gehören, Nicht-gemeintSein, Ausgeschlossen-Sein, Sich-Ermächtigen und Handlungsfähig-Sein) führen, die

5 Diese These wirkt, angesichts der in der Sequenz explizit geforderten Arbeitshaltung „still und leise“ zu arbeiten, widersprüchlich. Allerdings zeigt sich, sowohl in dieser Sequenz als auch über alle Einzelarbeitsphasen im videographierten Material hinweg, dass die Schüler_innen wie selbstverständlich miteinander dauerhaft kommunizieren und dies auch von den Lehrer_innen nicht kommentiert wird. Wann die Grenze überschritten wird, ab der sie einschreiten, variiert bei den einzelnen Lehrer_innen stark. 
durch Ermöglichung und Verschließung der Handlungsfähigkeit hergestellt werden und diese gleichzeitig bedingen.

\section{Autorenangaben}

Prof'in Dr. Alexandra Flügel

Department Erziehungswissenschaft und Psychologie

(Fakultät II: Bildung - Architektur - Künste)

Universität Siegen

alexandra.fluegel@universität-siegen.de

\section{Literatur}

Balzer, Nicole/Ricken, Norbert (2010): Anerkennung als pädagogisches Problem. Markierungen im erziehungswissenschaftlichen Diskurs. In: Schäfer, A./Thompson, C. (Hrsg.): Anerkennung. Paderborn: Ferdinand Schöningh Verlag, S. 35-87.

Bennewitz, Hedda (2009): Zeit zu Zetteln! - Eine Praxis zwischen Peer- und Schülerkultur. In: de Boer, H./Deckert-Peaceman, H. (Hrsg.): Kinder in der Schule. Zwischen Gleichaltrigenkultur und schulischer Ordnung. Wiesbaden: VS Verlag, S. 119-136.

Bennewitz, Hedda/Meier, Michael (2010): Zum Verhältnis von Jugend und Schule. Ethnographische Studien zu Peerkultur und Unterricht. In: Brake, A./Bremer, H. (Hrsg.): Alltagswelt Schule. Die soziale Herstellung schulischer Wirklichkeit. Weinheim: Juventa Verlag, S. 97-110.

Bennewitz, Hedda/Breidenstein, Georg/Meier, Michael (2015): Zum Verhältnis von Peerkultur und Schulkultur. In: Böhme, J./Hummrich, M./Kramer, R.-T. (Hrsg.): Schulkultur. Theoriebildung im Diskurs. Wiesbaden: Springer Verlag, S. 285-305.

de Boer, Heike (2009): Peersein und Schülersein - ein Prozess des Ausbalancierens. In: de Boer, H./Deckert-Peaceman, H. (Hrsg.): Kinder in der Schule. Zwischen Gleichaltrigenkultur und schulischer Ordnung. Wiesbaden: VS Verlag, S. 105-118.

Bollig, Sabine/Kelle, Helga (2014): Kinder als Akteure oder als Partizipanden von Praktiken? Zu den Herausforderungen für eine akteurszentrierte Kindheitssoziologie durch Praxistheorien. In: Zeitschrift für Soziologie der Erziehung und Sozialisation 34, 3, S. 263-279.

Breidenstein, Georg (2004): KlassenRäume - eine Analyse räumlicher Bedingungen und Effekte des Schülerhandelns. In: Zeitschrift für qualitative Bildungs-, Beratungs- und Sozialforschung 5, 1, S. 87-107.

Breidenstein, Georg (2006): Teilnahme am Unterricht. Ethnographische Studien zum Schülerjob. Wiesbaden: VS Verlag.

Breidenstein, Georg/Hirschauer, Stefan/Kalthoff, Herbert/Nieswand, Boris (2015): Ethnografie. Die Praxis der Feldforschung. 2. Aufl. Konstanz: UTB.

Budde, Jürgen (2015): Reflexionen zur Bedeutung von Handlung und Praktik in der Ethnographie. In: Zeitschrift für Qualitative Forschung 16, 1, S. 7-24.

Deppermann, Arnulf (2008): Gespräche analysieren. Eine Einführung. 4. Aufl. Wiesbaden: VS Verlag.

Dinkelaker, Jörg/Herrle, Matthias (2009): Erziehungswissenschaftliche Videographie. Eine Einführung. Wiesbaden: VS Verlag.

Eckermann, Torsten/Heinzel, Friederike (2015): Kinder als Akteure und Adressaten? - Praxistheoretische Überlegungen zur Konstitution von Akteuren und (Schüler-)Subjekten. In: Zeitschrift für Soziologie der Erziehung und Sozialisation 35, 1, S. 23-38. 
Fritzsche, Bettina (2015): Wenn niemand zu Schaden kommen darf: Eine kulturvergleichende Analyse schulischer Praktiken der Konfliktbearbeitung. In: Zeitschrift für Qualitative Forschung 16, 2, S. 173-190.

Fritzsche, Bettina/Idel, Till-Sebastian/Rabenstein, Kerstin (2011): Ordnungsbildung in pädagogischen Praktiken. Praxistheoretische Überlegungen zur Konstitution und Beobachtung von Lernkulturen. In: Zeitschrift für Soziologie der Erziehung und Sozialisation 31, 1, S. 28-44.

Herrle, Matthias/Dinkelaker, Jörg (2016): Qualitative Analyseverfahren in der videobasierten Unterrichtsforschung. In: Rauin, U./Herrle, M./Engartner, T. (Hrsg.): Videoanalysen in der Unterrichtsforschung. Weinheim: Beltz Juventa Verlag, S. 76-129.

Hertel, Thorsten/Pfaff, Nicole (2015): Studien zur Konstruktion sozialer Klassenzugehörigkeit im schulischen Feld - eine Perspektive der Bildungsgleichheitsforschung. In: Bräu, K./ Schlickum, C. (Hrsg.): Soziale Konstruktionen in Schule und Unterricht. Zu den Kategorien Leistung, Migration, Geschlecht, Behinderung, Soziale Herkunft und deren Interdependenzen. Opladen: Budrich, S. 263-278.

Hirschauer, Stefan (2014): Un/doing Differences. Die Kontingenz sozialer Zugehörigkeiten. In: Zeitschrift für Soziologie 43, 3, S. 170-191.

Hnilica, Sonja (2010): Schulbank und Klassenzimmer - Disziplinierung durch Architektur. In: Egger, R./Hackl, B. (Hrsg.): Sinnliche Bildung. Pädagogische Prozesse zwischen vorprädikativer Situierung und reflexivem Anspruch. Wiesbaden: VS, S. 141-162.

Knoblauch, Hubert (2001): Fokussierte Ethnographie. Soziologie, Ethnologie und die neue Welle der Ethnographie. In: Sozialer Sinn 2, 1, S. 123-141.

Rabenstein, Kerstin/Steinwand, Julia (2016): Praktiken der Differenz(re)produktion im individualisierten Unterricht: Ethnographische Videobeobachtungen. In: Rauin, U./Herrle, M./Engartner, T. (Hrsg.): Videoanalysen in der Unterrichtsforschung. Weinheim: Beltz Juventa Verlag, S. 242-262.

Reh, Sabine (2012): Mit der Videokamera beobachten. Möglichkeiten qualitativer Unterrichtsforschung. In: de Boer, H./Reh, S. (Hrsg.): Beobachtung in der Schule - Beobachten lernen. Wiesbaden: VS Verlag, S. 151-169.

Reh, Sabine/Ricken, Norbert (2012): Das Konzept der Adressierung. Zur Methodologie einer qualitativ-empirischen Erforschung von Subjektivation. In: Miethe, I./Müller, H.-R. (Hrsg.): Qualitative Bildungsforschung und Bildungstheorie. Opladen: Verlag Barbara Budrich, S. 35-56.

Ricken, Norbert (2015): Pädagogische Professionalität - revisited. Eine anerkennungstheoretische Skizze. In: Böhme, J./Hummrich, M./Kramer, R.-T. (Hrsg.): Schulkultur. Theoriebildung im Diskurs. Wiesbaden: VS Verlag, S. 137-157.

Ricken, Norbert (2016): „Streit trennt, Streit verbindet“. Das Problem der Heterogenität zwischen Immunisierung und Communisierung. In: Dogmus, A./Karakasoglu, Y./Mecheril, P. (Hrsg.): Pädagogisches Können in der Migrationsgesellschaft. Wiesbaden: VS Verlag, S. 43-58.

Rose, Nadine/Gerkmann, Anna (2015): Differenzierung unter Schüler_innen im reformorientierten Sekundarschulunterricht - oder: warum wir vorwiegend ,Leistung' beobachten, wenn wir nach ,Differenz‘ fragen. In: Zeitschrift für Qualitative Forschung 16, 2, S. 191-210.

Schicke, Christiane (2007): Oder doch lieber im Hufeisen? Vom Experimentieren mit Sitzordnungen. In: Grundschule 10, S. 18-21.

Schütz, Anna (2015): Schulkultur und Tischgemeinschaft. Eine Studie zur sozialen Situation des Mittagsessen an Ganztagsschulen. Wiesbaden: VS Verlag.

Strauss, Anselm/Corbin, Juliet (2010): Grounded Theory. Grundlagen qualitativer Sozialforschung. Weinheim: Beltz PVU.

Weichsel, Felix (2014): Plädoyer für eine variable Sitzordnung. Wie sich didaktisch-methodische Unterrichtsansätze im Arrangement von Tischen und Stühlen wiederfinden lassen. In: Lernende Schule 17, 65, S. 22-24. 


\section{Zwischen Mitgliedschaft und Teilhabe. Praxeologische Rekonstruktionen von Teilhabeformen im inklusiven Unterricht}

\section{Zusammenfassung}

Mit der Umstellung auf Inklusion wird Schulen eine weitgehende Teilhabe aller Schüler_innen vorgegeben. Im Unterricht inklusiver Klassen entstehen Variationen von Teilhabe, die sämtlich auf eine temporäre Reduktion von Anforderungen für bestimmte Schüler_innen verweisen. Reduzierte Anforderungen zeigen sich sowohl in unterrichtlichen als auch in organisationalen Praktiken sowie in diskursiven Verständigungen der Pädagog_innen. Trotz der verbrieften Mitgliedschaft wird Teilhabe paradoxerweise durch partielle Nicht-Teilhabe sichergestellt. Die damit einhergehende Ambivalenz von Schonung und Ausschluss deutet auf ein im Unterricht inklusiver Klassen hervorgebrachtes institutionalisiertes Adressierungsmuster hin, das Mitgliedschaft aller Schüler_innen unter Bedingungen reduzierter Teilhabe gewährleistet.

Schlagwörter: Inklusionsforschung, teilnehmende Beobachtung, Praxistheorie, Unterricht, Differenz

\section{Between Membership and Participation. Praxeological Reconstructions of Participation in In- clusive Classrooms}

Due to inclusion, schools are required to enable participation of all pupils. In inclusive classes variations of participation arise. So far, forms of participation refer to a temporary reduction of requirements for certain pupils. Reduced requirements are reflected in teaching and in organizational practices as well as in discursive understandings of the teachers. Despite the given membership, paradoxically, participation for certain pupils is ensured through partial nonparticipation. The resulting ambivalence of caring and exclusion points out an institutionalized addressing pattern, which ensures membership under conditions of reduced participation. Keywords: Inclusion Research, Participatory Observation, Practice Theory, Classes, Difference

Die bildungspolitische Ausgangssituation in Nordrhein-Westfalen erfährt mit der 9. Schulrechtsänderung seit dem Schuljahr 2014/15 insofern eine Zäsur, als gemeinsames Lernen aller Schüler_innen mit und ohne sonderpädagogischem Förderbedarf nun als Regelfall vorgegeben wird (vgl. Ausschuss für Schule und Weiterbildung des Landtags Nordrhein-Westfalen 2014: $§ 20$ Abs. 2). Wie in anderen Bundesländern auch ist die Rechtslage dahingehend eindeutig, dass im Zuge der Umsetzung von Inklusion eine Veränderung der Schulstruktur angestrebt wird, die auf Formen der äußeren Differenzierung weitgehend verzichtet und die Teilhabe aller Schüler_innen programmatisch vorgibt. Zum Prinzip wird die innere Differenzierung, ,realisiert in Interaktion und gestützt auf (...) Ziele, Praktiken und Materialien“ von Inklusion (Tenorth 2013: 10). Dabei bleibt unbestimmt, wie gemeinsames Lernen im inklusiven Unterricht umgesetzt werden kann. Wie in anderen Transformationsprozessen auch (vgl. Reh et al. 2015), stehen 
schulische Akteure vor der Herausforderung, Inklusion ${ }^{1}$ für ihre Unterrichtspraxis zu adaptieren und (weiter) zu entwickeln.

Der vorliegende Beitrag rekonstruiert in praxeologischer Perspektive (vgl. Schatzki 1996, 2002; vgl. Schatzki/Knorr-Cetina/Savigny 2001), welche Formen von Teilhabe in inklusiven Klassen zur Aufführung kommen und welche (impliziten) Handlungslogiken diesen Teilhabeformen zugrunde liegen. Dabei fokussieren wir die performative Modulation von Teilhabe, die systematisch über Aushandlungsprozesse der Nicht-Teilhabe ${ }^{2}$ in den Blick gerät. Wir nehmen Bezug auf Studien zum doing difference, welche auch in inklusiven Lernsettings auf Momente von Exklusion aufmerksam machen (vgl. Blasse et al. 2014) und etwa auf Marginalisierungen in Praktiken der Fürsorge (vgl. Fritzsche 2014) verweisen. ${ }^{3}$ In unseren Rekonstruktionen geraten dabei vorrangig die so benannten „sayings“ (Schatzki 2002), also die Sprachlichkeiten der Praktiken, in den Blick.

Unser empirisches Material ist im Kontext des Forschungsprojekts „EFiS-NRW Auf dem Weg zur Inklusion: Ethnographische Feldstudien in Schulen in NRW“ entstanden, welches wir zunächst kurz vorstellen (Abschnitt 1). Anschließend wird aufgezeigt, wie wir unterrichtliche Teilhabe in praxistheoretischer Perspektive konzeptualisiert haben (Abschnitt 2). Auf dieser Grundlage rekonstruieren wir anhand ausgewählter Beobachtungen unterrichtliche Teilhabeformen und ergänzen diese um Auszüge aus ethnographischen Interviews und ein schulisches Dokument (Abschnitt 3). Abschließend diskutieren wir, welche Subjektpositionen mit den rekonstruierten Teilhabeformen verbunden sind und ob sich davon ausgehend eine pädagogische Ordnung inklusiven Unterrichts konturieren lässt (Abschnitt 4).

\section{Das Forschungsprojekt EFiS-NRW}

Das Forschungsprojekt „EFiS-NRW - Auf dem Weg zur Inklusion: Ethnographische Feldstudien in Schulen in NRW“4 zielt darauf ab, aktuelle Herausforderungen, die mit Inklusion und deren konkreter Umsetzung in der schulischen Praxis zusammenhängen, ethnographisch zu erfassen. ${ }^{5}$ Damit wird eine Forschungsstrategie gewählt, die im Kontext von Inklusion bislang eher selten umgesetzt wird (vgl. Budde/Heynoldt/Offen 2016: 114; vgl. Merl/Winter 2014: 57). Die leitende Untersuchungsfrage des Projekts lautet: Wie rezipieren die beteiligten Schulen schulrechtliche und bildungspolitische

1 Auch im aktuellen (schul-)pädagogischen Diskurs zeigt sich ein divergentes Inklusionsverständnis (vgl. hierzu Sturm 2012; Budde/Hummrich 2013; Hazibar/Mecheril 2013; Cramer/Harant 2014; Werning 2014; Hinz 2015).

2 Wir sprechen von „Nicht-Teilhabe“ anstelle von „Ausschluss“ oder „Befreiung“ vom Unterricht, da unsere Rekonstruktionen den Vollzug der Praktiken fokussieren und nicht auf die Intentionen der Akteure zielen.

3 In Arbeiten zu individualisierendem Unterricht wird deutlich, dass über die implizite Norm der Selbständigkeit Differenzen zwischen Schüler_innen hervorgebracht werden, die dieser Norm entsprechen bzw. diese Norm nicht erfüllen (vgl. hierzu Reh 2013; Reh/Rabenstein 2012).

4 Das Projekt wird geleitet von Petra Herzmann, Argyro Panagiotopoulou und Lisa Rosen.

5 Für eine ausführlichere Darstellung des Forschungsprojekts und erste Ergebnisse vgl. Herzmann et al. (i.D.). 
Vorgaben zu Inklusion und welche Konzepte inklusiven Unterrichts entwickeln sie? Das Projekt EFiS-NRW erhebt Daten in verschiedenen Schulformen: Hierzu gehören eine Grundschule, eine Sekundarschule, eine private, staatlich anerkannte Gesamtschule und ein Gymnasium. Alle Schulen beschreiben sich oder zumindest einzelne Klassen ihrer Schule als ,inklusiv“. Der vorliegende Beitrag basiert auf Erhebungen von Thorsten Merl in einer Gesamtschule und einer Sekundarschule.

Neben der Priorisierung der teilnehmenden Beobachtung als zentraler Methode ethnographischer Unterrichtsforschung (vgl. Panagiotopoulou 2013: 771) werden Beobachtungen in der Pause und in Teambesprechungen aufgezeichnet. Darüber hinaus haben wir ethnographische Interviews im Sinne von „friendly conversations“ (Spradley 1979: 55ff.) durchgeführt sowie schulische Dokumente erfasst. An die von Diehm, Kuhn und Machold (2013: 47) vorgeschlagene Artefaktanalyse (vgl. Abschnitt 3.2) schließen wir insofern an, als wir nach Zusammenhängen zwischen performativ hervorgebrachten Praktiken im Unterricht, Äußerungen von Lehrerpersonen und Schuldokumenten fragen. Wir werten unsere Daten in Anlehnung an die Methodologie der Grounded Theory aus (vgl. Charmaz 2006). Um die Daten zu sichten, haben wir diese kodiert und ausgewählte Sequenzen bzw. Äußerungen und Dokumente, die auf die Verhandlung oder Begründung von Teilhabe verweisen, sequenziell interpretiert (vgl. Abschnitt 3). Dabei steht gegenwärtig - neben der weiteren axialen Kodierung und der Kategorisierung des (Beobachtungs-)Materials - die Relationierung von Konzepten unterrichtlicher Teilhabe im Fokus unserer Analysen. Für den vorliegenden Beitrag schlagen wir eine Systematisierung unterrichtlicher Teilhabeformen vor, die - wie im Folgenden ausgeführt wird - zwischen institutionell verbriefter Mitgliedschaft und Teilhabe unterscheidet.

\section{Unterrichtliche Teilhabe in praxistheoretischer Perspektive}

In praxistheoretischer Perspektive werden Praktiken verstanden als ,,arrays of activity“ (Schatzki 2001: 11). Praktiken emergieren aus dem körperlich-sprachlichen Vollzug von Aktivitäten. Diese ,doings or sayings are ,linked“ by practical understanding“ (Schatzki 2002: 78). Sie sind als „collective action“ (Barnes 2001: 32) der „Ort des Sozialen“ (vgl. Reckwitz 2003: 286). Dabei besteht das Soziale einer Praktik in der „Repetitivität gleichartiger Aktivitäten über zeitliche und räumliche Grenzen hinweg, die durch ein praktisches Wissen ermöglicht wird“ (ebd.: 292). Damit von Teilhabe an bestimmten Praktiken gesprochen werden kann, bedarf es Aktivitäten, die „operativ aufeinander bezogen“ sind (Hillebrandt 2014: 59). Die singuläre Hervorbringung von „doings“ und „sayings“ wird noch nicht als Teilhabe an einer sozialen Praktik verstanden. „A doing or saying belongs to a given practice if it expresses components of that practice's organization“ (Schatzki 2002: 87). Eine einzelne Aktivität muss demnach Ausdruck eines Elements der gesamten Organisation einer Praktik sein, was wiederum geteiltes praktisches Verstehen, geteilte Regeln und geteilte (normative) Zwecke (vgl. Schatzki 2002: 77-86; ders. 2012: 15) voraussetzt. Ein Akteur wird „Beteiligter an einer Praxis, wenn er in seinen Aktivitäten der Organisiertheit der Praxis Ausdruck verleiht, an ihr mitwirkt“" (Reh et al. 2015: 302). 
Von diesen Überlegungen ausgehend verstehen wir unterrichtliche Teilhabe als Teilhabe an Praktiken, die Ausdruck geteilter Regeln der unterrichtlichen Akteure sind und von diesen sprachlich und körperlich im sozialen Gefüge der Schulklasse hervorgebracht werden. Teilhabe wird also nicht nur durch schulstrukturelle Zuweisungen bestimmt, wonach ein_e Schüler_in aufgrund der Beschulung am gemeinsamen Unterricht per se teilhat. Diese Art von Mitgliedschaft (vgl. Stichweh 2013) in einer Klasse ist zwar eine notwendige, nicht aber eine hinreichende Bedingung von Teilhabe. Teilhabe muss vielmehr über Praktiken performativ aufgeführt und in Aktivitäten des Teilnehmens prozessiert werden (vgl. Breidenstein 2006). Diese sprachlich-körperlichen Vollzüge sind der Beobachtung zugänglich, weshalb wir mittels ethnographischer Zugänge rekonstruieren, welche Akteure mittels welcher Aktivitäten an welchen Praktiken teilhaben und welche (impliziten) Anforderungen an Teilhabe gestellt werden. Dabei fokussieren wir vorrangig sprachliche Vollzüge.

Unter Berücksichtigung von Arbeiten zu Subjektivierung und Anerkennung kann der Vollzug von Praktiken weitergehend als Hervorbringung spezifischer Subjektpositionen verstanden werden (vgl. Balzer/Ricken 2010): „Menschen werden zu entsprechenden Subjekten gemacht, weil und indem sie Praktiken selbst ausüben. Subjektformierung bzw. Subjektivierung sind nicht nur mit Praktiken verbunden, sondern diesen inhärent" (Reh/Ricken 2012: 39). Dies ist insofern der Fall, als Subjektivierung durch Anerkennungsprozesse geschieht und sich Anerkennungsprozesse wiederum als Adressierungen in Praktiken zeigen lassen (vgl. ebd.). Demzufolge kann über die Rekonstruktion von Praktiken auch auf Ordnungen im inklusiven Unterricht geschlossen werden: Die Antwort auf die Frage, wer im Unterricht wie adressiert wird und als welches Subjekt am Unterricht teilhaben kann, konturiert Möglichkeiten des Teilnehmens im inklusiven Unterricht.

\section{Rekonstruktionen unterrichtsbezogener Teilhabeformen}

Wir betrachten im Folgenden von diesem Konzept der Teilhabe ausgehend solche Unterrichtssequenzen, in denen Teilhabe zwischen den Akteuren ausgehandelt wird. Ziel ist es, spezifische Formen von Teilhabe herauszuarbeiten, die sich im Unterricht der beobachteten Klassen zeigen. Wir unterscheiden dabei einerseits Teilhabeformen, die ein zeitweiliges Verlassen des Klassenraumes hervorbringen, und andererseits Teilhabeformen, in denen Schüler_innen zwar in der Klasse verbleiben, an der Bearbeitung des Unterrichtsgegenstandes zeitweise aber nicht partizipieren (vgl. Abschnitt 3.1). Diese beiden Teilhabeformen werden wir um einen ethnographischen Interviewauszug ergänzen, um aufzuzeigen, wie pädagogisch Professionelle eigene Praktiken und in ihnen eingelagerte Entscheidungen kommentieren und so Formen der Teilhabe und NichtTeilhabe begründen. Anhand der Hinzunahme einer tabellarischen Übersicht zu Fördernotwendigkeiten von Schüler_innen werden wir abschließend darstellen, wie durch Artefakte Möglichkeiten der Teilhabe und Nicht-Teilhabe manifestiert werden (vgl. Abschnitt 3.2). 


\subsection{Unterrichtliche Praktiken}

Das folgende Protokoll bezieht sich auf eine Unterrichtsszene einer sechsten Klasse an einer Sekundarschule:

„Während eines Klassengesprächs zum Umgang mit dem Gasbrenner sagt Herr Roland ${ }^{6}$ : ,Stopp, stopp mal kurz. Tom, schaffst du's oder brauchst du 'ne Auszeit?' Tom: ,Äh, brauch 'ne Auszeit.” Herr Roland: ,Gut, dann nimm sie dir. ' (...) Tom verlässt die Klasse. Sein Schulbegleiter folgt ihm. Sie kommen nach etwa 20 Minuten zurück.“ (Sekundarschule; 2014-06-02)

In diesem Auszug wird Tom vorübergehend aus dem Klassenraum entlassen. Auf Rückfrage erklärt Toms Schulbegleiter dem Ethnographen, dass Tom und er während der Auszeit „auf dem Klettergerüst“ des Schulgeländes waren. Das Klassengespräch wird von Herrn Roland nur kurz unterbrochen (,Stopp, stopp mal kurz“) und läuft dann ohne Tom weiter. Diese Unterbrechung steht in Zusammenhang mit seiner Frage an Tom, ob er es weiterhin schaffen kann, am Unterricht teilzunehmen, oder ob er eine Auszeit braucht. Tom bestätigt explizit, eine Auszeit zu brauchen. Aus dieser Bestätigung der Auszeit resultiert für Herrn Roland, Tom aus dem Klassenzimmer zu entlassen. Die Regulation mittels Auszeit durch Herrn Roland ist insofern eine prophylaktische, als diese dann (pädagogisch) sinnvoll erscheint, wenn angenommen wird, dass die mit der Teilhabe an unterrichtlichen Praktiken einhergehenden Anforderungen für Tom eine Überforderung darstellen.

Dass das Klassengespräch nur kurz unterbrochen wird, verweist außerdem darauf, dass es sich bei dieser Auszeit um eine Abweichung von allgemein gültigen Anforderungen handelt. Über die Ausnahme, die für Tom gemacht wird, werden die geltenden Anforderungen und die Abweichung von diesen verhandelt. Insofern stellt diese Auszeit eine zeitlich limitierte Dispensation der unterrichtlichen Anforderungen für Tom dar: Für die Dauer der Auszeit nimmt Tom nicht am Klassengespräch teil. Die vorübergehende Auszeit, verstanden als zeitlich begrenzte und durch räumliche Trennung vollzogene Nicht-Teilhabe, erscheint in der Logik der Praxis als Form der Teilhabe bei gleichzeitiger Nicht-Teilhabe. Paradoxerweise wird Teilhabe also in Form der vorübergehenden Nicht-Teilhabe aufrechterhalten und damit begründet, dass eine andere Form der Teilhabe nicht möglich ist. Das Protokoll gibt keinen zusätzlichen Hinweis darauf, ob dem dargestellten Auszug das Klassengespräch störende Tätigkeiten von Tom vorausgingen. Dennoch erfüllt die vorübergehende Auszeit auch die Funktion, potenzielle Störungen durch Tom zu vermeiden.

Diese Form der zusätzlichen Auszeit - neben den festgelegten Pausen - stellt im von uns beobachteten Unterricht insofern eine legitime Option für bestimmte Schüler innen dar, als dass Tom die Auszeit angeboten bekommt. Sie stellt keinen sanktionierenden Rauswurf dar, sondern bezeichnet im Kollektiv der Klasse ein geteiltes Verständnis einer lehrerseitigen Schonung gegenüber Schüler_innen, denen zugeschrieben wird, nicht (mehr) zu können. Eine Sanktionierung erscheint als unangemessen, weil bei der Zuschreibung von ,Nicht-mehr-Können“ nicht auf Motivation, sondern auf Fähigkeit attribuiert wird. Die Funktion dieser Auszeit ist demnach Regeneration, nicht Sanktion. Ausdruck des klassenöffentlichen Commitments ist auch, dass die Legitimität dieser

6 Die Namen aller Akteure sind aus Gründen der Anonymisierung geändert. 
pädagogischen Intervention über eine Delegation eingeholt wird: Nicht Herr Roland, sondern Tom soll entscheiden, ob er es schafft oder nicht. Dadurch verlagert sich die Begründungslast der Intervention von der Lehrkraft auf den Schüler und wird über dessen Zustimmung auch von ihm selbst als gültig anerkannt.

Der folgende zweite Auszug wurde im Unterricht einer jahrgangsübergreifenden Klasse der Stufen 5-7 an einer Gesamtschule protokolliert. Die Schüler_innen arbeiten in dieser Phase des Unterrichts an Wochenplänen. Frau Juhn, eine der beiden Klassenlehrerinnen, sitzt neben einem Schüler und bearbeitet mit ihm Aufgaben im Fach Deutsch.

„Frau Juhn schaut zu Leon und Sibo, die Mandalas malen. Andere Schüler_innen stehen daneben, schauen und reden miteinander. Dann sagt Frau Juhn: ,Ich möchte nicht, dass jetzt jeder zuguckt, wie Mandalas gemalt werden. Ihr habt was zu tun'. Dann dreht sie sich wieder zu Lucca und liest mit ihm weiter.“ (Gesamtschule; 2015-03-20)

Leon und Sibo bekamen etwa zwanzig Minuten vor dem zitierten Auszug angeboten, sich Motive für Mandalas auszusuchen und diese vorübergehend auszumalen, anstatt an den Aufgaben des Wochenplans zu arbeiten. Diese malen sie nun aus. Es ist in der Klasse üblich, dass Schüler_innen zur Organisation ihrer Arbeit an den Wochenplänen bspw. aufstehen und zu ihren Regalfächern gehen. Der Auszug macht zunächst die Gleichzeitigkeit unterschiedlicher Aktivitäten der Schüler_innen während der Wochenplanarbeit kenntlich: Einige Schüler_innen organisieren ihre Materialien, andere Schüler_innen bearbeiten ihre Aufgaben, ein Schüler arbeitet gemeinsam mit der Lehrerin. Diese Aktivitäten sind sämtlich Ausdruck der unterrichtlichen Praxis der Wochenplanarbeit ${ }^{7}$. Dass nun einige Schüler_innen stehen bleiben und Leon und Sibo beim Malen der Mandalas zuschauen, wird durch Frau Juhn als unerwünscht markiert. Sie ermahnt die Herumstehenden und fügt hinzu, dass diese schließlich etwas zu tun hätten. Das Herumstehen wird hierdurch als illegitime Aktivität der Schüler_innen während der Wochenplanarbeit kenntlich gemacht. Für Leon und Sibo gilt das Ausmalen der Mandalas als eine zulässige Aktivität während der Wochenplanarbeit. Im Kollektiv der Klasse wird somit öffentlich aufgeführt, was als legitim und was als illegitim gilt.

Ähnlich der gewährten Auszeit von Tom geht mit dem Malen von Leon und Sibo eine vorübergehende Entbindung von unterrichtlichen Anforderungen einher. Das Beobachtungsprotokoll macht deutlich, dass Leon und Sibo zwar am Unterricht teilhaben, eine Teilhabe an Praktiken, die auf den Wochenplan bezogen sind, momentan jedoch nicht stattfindet. Ebenso wie die regenerative Auszeit von Tom gilt auch diese Form der Beschäftigung als legitime Praktik. Während jedoch die Auszeit von Tom Ausdruck einer räumlichen Nicht-Teilhabe ist, verbleiben Leon und Sibo im Klassenraum. Die partielle Nicht-Teilhabe wird hier dadurch hervorgebracht, dass die Schüler von sachlichen Anforderungen des Unterrichts zeitweilig freigestellt werden. Teilhabe bleibt unter den Bedingungen partieller Nicht-Teilhabe im Sinne der Kopräsenz im Raum grundsätzlich aufrechterhalten.

Fasst man die unterrichtlichen Teilhabeformen bis hierhin zusammen, dann zeigt sich, dass es möglich ist, an bestimmten unterrichtlichen Praktiken teilzuhaben und an

7 Zu Rekonstruktionen der sozialen Ordnung des Wochenplanunterrichts vgl. Reh/Labede (2009). 
anderen Praktiken nicht. Performativ entstehen Figurationen von Teilhabe, die über die schlichte Dualität von Teilhabe vs. Nicht-Teilhabe hinausgehen. Zwischen Mitgliedschaft und weitgehender Teilhabe an unterrichtlichen Praktiken entstehen Variationen einer „exkludierenden Inklusion“ (Stichweh 2013). Differenzierungen können in Bezug auf sachliche, soziale und zeitliche Prozesse des Teilnehmens vorgenommen werden: ${ }^{8}$ So besteht die Reduktion von sachbezogenen Anforderungen etwa darin, die Beschäftigung mit dem Unterrichtsgegenstand zeitweilig auszusetzen. Das Verlassen des Klassenraums und die Bereitstellung von Extra-Aufgaben verweisen auch auf die soziale Dimension der Teilhabepraktiken, da sie klassenöffentlich verhandelt werden. Nicht zuletzt werden sämtliche Formen der Nicht-Teilhabe über die Zeit reguliert. In der Logik der Praxis ist die partielle Nicht-Teilhabe aber nur für jene Schüler_innen legitim, denen lehrerseitig zugeschrieben wird, zumindest phasenweise die allgemein gültigen Anforderungen des Unterrichts nicht erfüllen zu können. Wenn also aufgrund der schulrechtlichen Vorgabe der inklusiven Beschulung die Mitgliedschaft aller Schüler_innen organisational zwar festlegt ist, so bleibt Teilhabe an den gemeinsamen Aktivitäten im Unterricht limitiert.

\subsection{Diskurse und Artefakte}

Aufgrund der Schwierigkeit einer „Situationszentrierung“ (Diehm/Kuhn/Machold 2013: 42ff.) des ethnographischen Paradigmas wird vorgeschlagen, Beobachtungsprotokolle um zusätzliche Materialsorten zu erweitern. Zur Kontextualisierung des Situativen können ,aneinander gekoppelte Aggregatzustände“ (Reckwitz 2008: 202) des Sozialen - Praktiken, Diskurse, Artefakte, etc. - aufeinander bezogen und an das Material herangetragen werden. Das folgende Beobachtungsprotokoll verweist darauf, wie die Lehrkräfte Frau Juhn und Herr Terra, die gemeinsam eine jahrgangsübergreifende Lerngruppe leiten, eigene Praktiken und in ihnen eingelagerte Entscheidungen begründen. In der protokollierten Sequenz sitzen sie vor dem Beginn der ersten Unterrichtsstunde an ihren Plätzen im Lehrerzimmer und schauen in Unterlagen, die auf ihrem Schreibtisch liegen.

„Ich bekomme mit, dass Frau Juhn Herrn Terra zwei Bücher zeigt. Dabei sagt sie, dass, wenn Leon heute nicht mehr könne, er entweder Mandala malen oder das Fußballbuch (beides hält sie in den Händen) bekommen solle. Herr Terra nickt daraufhin.“ (Gesamtschule; 2015-04-13)

Frau Juhn, die Sonderpädagogin, spricht Herrn Terra, den Regelschullehrer, mit zwei Büchern in der Hand an. Sie erklärt ihm, dass diese für Leon seien. Das Fußballbuch scheint eine Art Comic zu sein, das zweite Buch enthält Kopiervorlagen für das Ausmalen von Mandalas. Diese Bücher sollen dann zum Einsatz kommen, wenn Leon „heute nicht mehr könne". Sie stellen somit eine Alternative zu den geplanten und für die Klasse geltenden sachbezogenen Anforderungen dar. Bedingung für die Gewährung alternativer Tätigkeiten ist die vage Zuschreibung der Lehrpersonen von ,Nicht-mehr-Können“. Unbenannt bleibt etwa, was ,Nicht-Können“ konkret bedeutet und woran der Zeitpunkt des ,Nicht-mehr-Könnens' festgestellt werden kann. Dieses diffuse Deutungsmuster

8 Für die Unterscheidung einer Sach-, Sozial- und Zeitdimension des Unterrichts vgl. auch Meseth/Proske/Radtke (2011). 
wird im Gespräch der Lehrkräfte insofern aufrechterhalten, als dass keine weitere Explikation des Gemeinten stattfindet. Dass die Lehrkräfte sich trotzdem verstehen, verweist vielmehr auf ein kollektives Wissen. So macht der kurze Hinweis auf die Bücher, der von Herrn Terra mit einem Nicken quittiert wird, kenntlich, dass die vorübergehende Aufhebung von Anforderungen eine gängige Praxis darstellt. Die vorsorgliche Bereitstellung alternativer Tätigkeiten macht überdies deutlich, dass zumindest die Sonderpädagogin die Notwendigkeit der Anforderungsreduktion bei bestimmten Schüler_innen regelhaft antizipiert. Zudem wird hier erneut kenntlich, dass fachliche Anforderungen nur vorübergehend ausgesetzt werden. Auch Leon hat Arbeitsaufgaben erhalten.

Der folgende Auszug aus einem ethnographischen Interview verweist ebenfalls auf diese Argumentation der Pädagog_innen. Es hat sich während einer Pause ereignet:

„In einem kurzen Gespräch mit Herrn Roland erklärt mir dieser, dass sie das Beantragen von sonderpädagogischem Förderbedarf immer ,Krankschreiben“ nennen.“ (Sekundarschule; 2014-06-02)

Eine Krankschreibung ist ein arbeitsrechtliches Instrument, das einen formalen Status herstellt, der berufliche Anforderungen vorübergehend aussetzt und eine zeitweilige Arbeitsunfähigkeit attestiert. „Eigentlich“ bestehende Anforderungen werden für die Dauer der Krankschreibung gänzlich aufgehoben. Darüber hinaus ist das Instrument der Krankschreibung in der Regel auf ein Ende, die Gesundung, bezogen - im anderen Falle werden die Arbeitnehmer_innen dauerhaft als berufs- bzw. erwerbsunfähig statuiert. In der Bezeichnung ,Krankschreiben“ zeigt sich, wie Herr Roland die Beantragung des sonderpädagogischen Förderbedarfs versteht: Er verwendet den Begriff ,Krankschreibung' und das schulrechtliche Konstrukt der Beantragung des sonderpädagogischen Förderbedarfs synonym. Herr Roland markiert zugleich, dass die Bezeichnung ,Krankschreiben“ von seinen Kolleg_innen geteilt wird. Damit wird erneut auf ein kollektives Deutungsmuster der Professionellen hingewiesen. An dieser Stelle zeigt sich, in Analogie zum Vollzug pädagogischer Praktiken im Unterricht, eine temporäre Reduktion der unterrichtlichen Anforderungen für bestimmte Schüler_innen, die über das Diagnostikum des sonderpädagogischen Förderbedarfs bereitgestellt werden. Während die zusätzliche Auszeit Tom vorübergehend von den Anforderungen des Klassengesprächs entbindet und das Malen die fachlichen Anforderungen der Wochenplanarbeit für Leon und Sibo zeitweilig aufhebt, verweisen die Diskurse der Pädagog_innen auf die Zuschreibung einer temporär eingeschränkten Leistungsfähigkeit bestimmter Schüler innen, deren Schonung damit legitimiert wird. Jenseits des Vollzugs der unterrichtlichen Praktiken ist die Regulation von Teilhabe also auch über Begründungsfiguren der Pädagog_innen und auf der Ebene der organisationalen Praktiken des Diagnoseschreibens angesiedelt. Anders als bei einer arbeitsrechtlichen Krankschreibung sind die Schüler_innen aber nicht in vollem Umfang von der Teilhabe ausgeschlossen. Im schulischen Kontext handelt es sich mit der Beantragung des sonderpädagogischen Förderbedarfs deshalb um ein paradoxes Konstrukt einer relativ dauerhaften Krankschreibung, die zeitlich befristete und changierende Formen der Nicht-Teilhabe unter Aufrechterhaltung der Anwesenheit in der Schule ermöglicht und zugleich pädagogisch begründet. Zugleich (re-)produzieren die Lehrkräfte mit der Bezeichnung ,Krankschreibung' ein medizinisch ontologisches Verständnis von Behinderung, das Nicht-Teilhabe 
der Schüler innen individualisiert.

Beim folgenden Artefakt handelt es sich um ein Dokument, das dem Ethnographen mit der Beschreibung übergeben wurde, es sei eine Übersicht, ,alle[r] Schüler, die einen Förderschwerpunkt haben“. Darauf sind zehn Schüler_innen jeweils mit Foto, Namen und kurzen Beschreibungen abgebildet. Es wurde von der für die Lerngruppe zuständigen Sonderpädagogin erstellt. Der folgende, bildlich anonymisierte Auszug aus dieser Übersicht bezieht sich auf den Schüler Jan:

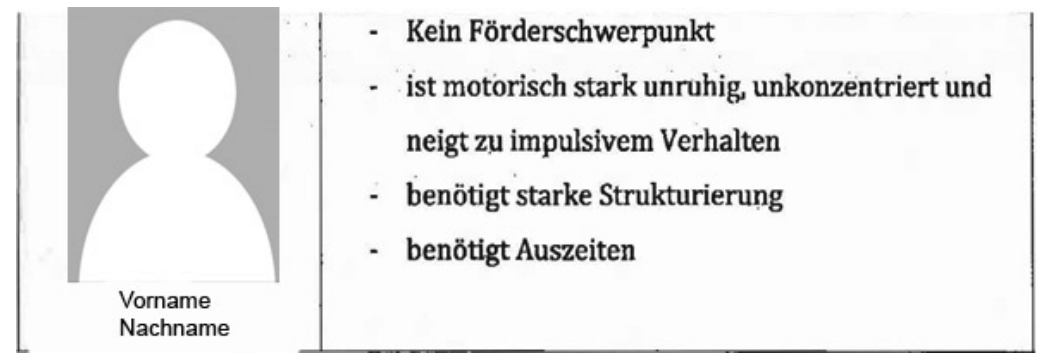

Abbildung 1: Übersicht

Zunächst einmal wird Jan ,kein Förderschwerpunkt“ zugeschrieben. Er ist damit der einzige Schüler, der ohne formal zugeschriebenen Förderbedarf (nach Schulgesetz NRW) auf der Übersicht erscheint. Als zweiter Punkt folgt eine Verhaltenszuschreibung: Jan ist ,motorisch stark unruhig, unkonzentriert und neigt zu impulsivem Verhalten“. Abschließend werden Unterstützungsbedarfe aufgeführt (,,benötigt ...“).

Geht man davon aus, dass die Zuschreibung der vermeintlichen Bedarfe in einem Zusammenhang mit der Verhaltensbeschreibung steht, dann wird deutlich, wie aus der Zuschreibung eines bestimmten Verhaltens reduzierte Anforderungen resultieren: Weil Jan unruhig und unkonzentriert ist, braucht er Struktur und Auszeiten. Jan wird durch das Artefakt als ein Schüler ausgewiesen, der zwar formal keinen Förderschwerpunkt hat, jedoch Verhaltensmerkmale aufweist, denen durch partielle Nicht-Teilhabe in Form von Auszeiten begegnet werden kann. Dass hier ein Schüler ohne formal zugeschriebenen sonderpädagogischer Förderbedarf aufgelistet ist, macht deutlich, dass die spezifischen Formen der partiellen Nicht-Teilhabe an unterrichtlichen Praktiken nicht ausschließlich entlang eines formal zugeschriebenen sonderpädagogischen Förderbedarfs organisiert sind. Der Zusammenhang von sonderpädagogischem Förderbedarf und Teilhabeformen lässt sich deshalb als systematisch, nicht aber als deterministisch bezeichnen.

Insgesamt verweisen auch die Kontextualisierungen auf die zugrundeliegende Handlungslogik der legitimen Reduktion und Dispensation von Anforderungen als spezifische Teilhabeformen für bestimmte Schüler_innen. Das Artefakt wird auf dem Schulserver auf unbestimmte Zeit zur Verfügung gestellt. Damit werden eingeschränkte Teilhabeformen in der Ambivalenz von Förderbedürftigkeit einerseits und Unterstützungsangeboten andererseits manifestiert. Auch der Begriff der ,Krankschreibung' markiert im Zusammenhang mit dem schulrechtlichen Konstrukt des sonderpädagogischen Förderbedarfs die Verständigung der Pädagog_innen auf die Reduktion von Anforde- 
rungen als Schonung für bestimmte Schüler_innen, die zugleich Ausdruck der ihnen zugeschriebenen eingeschränkten Leistungsfähigkeit sind. Zusammenfassend lässt sich konstatieren, dass die Teilhabeformen der partiellen Nicht-Teilhabe in unterrichtlichen Praktiken hervorgebracht werden, diese aber durch Verständigungen der Pädagog_innen sowie durch organisationale Praktiken des Diagnostizierens und der Förderplanung verstetigt werden, wie dies in der Bezeichnung ,Krankschreibung“ pointiert zum Ausdruck kommt.

\section{Diskussion: Teilhabe im Modus der ,Krankschreibung“}

Unsere Rekonstruktionen inklusiven Unterrichts zeigen, dass bei bestimmten Schüler innen unterrichtliche Teilhabe aufgrund eines zugeschriebenen Mangels (,Nicht-mehrKönnen') als Teilhabe unter reduzierten und vorübergehend dispensierten Anforderungen hervorgebracht wird. Zwischen Mitgliedschaft im Unterricht und weitreichender Teilhabe an unterrichtlichen Praktiken entstehen Formen der Teilhabe, die sich entlang der sachlichen, der sozialen und der zeitlichen Ordnung des Unterrichts (vgl. Meseth/ Proske/Radtke 2011) differenzieren lassen. So ist in der rekonstruierten Logik der Praxis, beispielsweise in Form regenerativer Auszeiten, für bestimmte Schüler_innen nur eine körperlich-räumliche, sachlich-inhaltliche und zeitlich eingeschränkte Form der Teilhabe möglich. Diese zeitweilige Nicht-Teilhabe wird von den Pädagog_innen als Schonung bestimmter Schüler_innen pädagogisch begründet. Ihre partielle Nicht-Teilhabe stellt in diesem Sinne eine legitime Praxis im inklusiven Unterricht dar.

Die Regelhaftigkeit der Teilhabeformen (vgl. Reh/Rabenstein/Idel 2011) zeigt sich zum einen in den wiederholt beobachtbaren Praktiken im inklusiven Unterricht. Zum anderen wird Regelhaftigkeit im kollektiven Wissen von Schüler_innen und Pädagog_innen sichtbar: So macht etwa die Verständigung der Lehrkräfte über alternative Beschäftigungsangebote deutlich, dass aus der Zuschreibung von ,Nicht-Können“ zugleich Schonung in der Erledigung sachlicher Anforderungen und Ausschluss aus der Beschäftigung mit dem Unterrichtsgegenstand resultieren. Die Rückfrage eines Lehrers an seinen Schüler, ob er dessen Einschätzung, den unterrichtlichen Anforderungen nicht mehr genügen zu können, teilt, hat nach dessen signalisiertem Einvernehmen eine regenerative Auszeit auf dem Schulhof zur Folge. Mit Hilfe von Artefakten, die auf organisationale Praktiken der Pädagog_innen verweisen, kann insofern eine weitergehende Gültigkeit beansprucht werden, als nicht nur die Ebene des Vollzugs pädagogischer Praktiken berührt ist. Vielmehr geben sowohl die Begründungsfiguren der Pädagog_innen für Praktiken als auch die schriftlichen Dokumentationen von Förderbedarfen, wie diese etwa in der Übersicht der Förderschüler_innen kenntlich werden, Hinweise auf ordnungsbildende Strukturen im inklusiven Unterricht.

Diese Teilhabeformen bezeichnen wir in Orientierung am in-vivo-Kode ,Krankschreibung' als Teilhabe im Modus der Krankschreibung, um von den regelhaften Figurationen der partiellen Nicht-Teilhabe ausgehend auf ein der pädagogischen Ordnung inhärentes Adressierungsmuster hinzuweisen: Entlang der Logik ,Krankschreibung ‘ werden Schüler_innen regelhaft als nicht hinreichend fähig für die mit der Teilhabe am Unterricht einhergehenden Anforderungen adressiert. Es finden sich partielle Teil- 
habeformen einerseits als Kopräsenz im Klassenraum, bspw. in Form der fachlichen Anforderungsreduktion, und andererseits als zeitweiliger Ausschluss aus dem Klassenraum. Diese Formen differenzierter Teilhabe sind mit Bezug auf den der Arbeitswelt entlehnten Krankheitsstatus pädagogisch begründbar, wenngleich damit auch die reduzierte Leistungsfähigkeit bestimmter Schüler_innen ontologisch festgeschrieben wird. In diesem Sinne wird eine in Praktiken inklusiven Unterrichts einnehmbare Subjektposition beschreibbar, die einerseits einen Mangel, andererseits eine legitime Form der Schonung impliziert. Die in dieser Weise ,krankgeschriebenen' Schüler_innen nehmen aufgrund der Zuschreibung mangelnder Fähigkeiten demnach als nur begrenzt teilhabefähige Schüler_innen teil. Zugleich bleibt aber die Mitgliedschaft aller Schüler_innen im inklusiven Unterricht garantiert. Dass es sich in der pädagogischen Ordnung inklusiven Unterrichts um ein institutionalisiertes Adressierungsmuster (vgl. Rabenstein/Idel/ Ricken 2015) handelt, zeigt sich am deutlichsten in den lehrerseitigen Bezugnahmen auf die schulrechtlich für inklusive Klassen relevante Kategorie des sonderpädagogischen Förderbedarfs.

Davon ausgehend stellt sich nun die Frage, inwiefern ein verändertes Ordnungsprinzip inklusiven Unterrichts entsteht, das dauerhafte Mitgliedschaft trotz limitierter Teilhabe im Modus der ,Krankschreibung' impliziert. Inwiefern entsteht im inklusiven Unterricht eine Schonung für bestimmte Schüler_innen, die diese zwar als Besondere positioniert, ohne aber ihre Mitgliedschaft in Frage zu stellen? Unsere Rekonstruktionen verweisen darauf, dass vor dem Hintergrund der verbrieften Mitgliedschaft im inklusiven Unterricht für jene ,krankgeschriebenen“ Schüler_innen phasenweise die Teilhabe an Praktiken, die sich im engeren Sinne auf den Unterrichtsgegenstand beziehen, in den Hintergrund treten. Inwieweit der regelhafte Ausschluss vom gegenstandsbezogenen Lernen die Teilhabe einzelner Schüler_innen lediglich auf deren Beschäftigt-Sein begrenzt, muss weitergehend untersucht werden. Ebenso bleibt die Frage unbeantwortet, inwiefern Auszeiten die Funktion erfüllen, dass sie Mitschüler_innen in ihrem Lernprozess störungsfreier arbeiten lassen.

Letztlich wird sich die Konturierung einer veränderten Unterrichtsordnung inklusiver Klassen an der „Gretchenfrage“ der Leistungsordnung bemessen müssen (vgl. Rabenstein et al. 2013). In unserem Material zeigt sich bisher, dass Leistungsanforderungen ${ }^{9}$ im Unterricht zwar reduziert werden und ,krankgeschriebene' Schüler_innen im Hinblick auf Leistungsanforderungen geschont werden, sie dadurch aber zugleich $\mathrm{Zu}$ - und Festschreibungen reduzierter Leistungsfähigkeit erfahren. Damit kann an das in der Differenzforschung bekannte Dilemma von Anerkennung und Reproduktion von Differenz (vgl. Kiesel 1996; vgl. Fritzsche 2014) angeknüpft werden. Die Ambivalenz von Schonung und Ausschluss bestimmter Schüler_innen wäre demnach eben nicht spezifisch für inklusiven Unterricht. In Orientierung daran machen unsere Arbeiten aber darauf aufmerksam, dass die Gleichzeitigkeit und Gleichförmigkeit schülerseitiger Verhaltens- und Lernprozesse gerade auch im inklusiven Unterricht eine empirische Illusion sind. Die Konturierung einer spezifischen Ordnung inklusiven Unterrichts wäre demnach obsolet und es käme vielmehr darauf an, die Verschiebung im Umgang mit

9 Leistung verstehen wir in einem umfassenden Sinne nicht nur auf Kognition bezogen, sondern u. a. auch auf Motivation, Disziplin und Schnelligkeit des Lernens (vgl. hierzu exemplarisch Ricken 2014: 127). 
Differenz bei schulrechtlich vorgegebener Mitgliedschaft aller Schüler_innen im inklusiven Unterricht weitergehend auszudifferenzieren.

\section{Autorenangaben}

Prof. Dr. Petra Herzmann

M.A. Thorsten Merl

Universität zu Köln

Fachgruppe Erziehungs- und Sozialwissenschaften

Institut für Allgemeine Didaktik und Schulforschung, Humanwissenschaftliche Fakultät

officeherzmann@uni-koeln.de

thorsten.merl@uni-koeln.de

\section{Literaturverzeichnis}

Ausschuss für Schule und Weiterbildung des Landtags Nordrhein-Westfalen (2014): Schulgesetz für das Land Nordrhein-Westfalen. [https://www.schulministerium.nrw.de/docs/Recht/ Schulrecht/Schulgesetz/Schulgesetz.pdf], Zugriff am 30.11.2016.

Balzer, Nicole/Ricken, Norbert (2010): Anerkennung als pädagogisches Problem - Markierungen im erziehungswissenschaftlichen Diskurs. In: Schäfer, A./Thompson, C. (Hrsg.): Anerkennung. Paderborn u. a.: Schöningh, S. 35-87.

Barnes, Barry (2001): Practice as collective action. In: Schatzki, T. R./Knorr-Cetina, K./Savigny, E. v. (Hrsg.): The practice turn in contemporary theory. New York: Routledge, S. 25-36.

Blasse, Nina/Budde, Jürgen/Hinrichsen, Merle/Hummrich, Merle/Niemeyer-Jensen, Beatrix/ Thon, Christine (2014): Die Exklusivität des Inklusiven. In: Schulpädagogik heute 5, 10. Onlinezeitschrift ohne Seitenangaben. Wiederveröffentlicht 2015 in: Siedenbiedel, C./ Theurer, C. (Hrsg.): Grundlagen inklusiver Bildung, Teil 2. Entwicklung zur inklusiven Schule und Konsequenzen für die Lehrerbildung. Immenhausen bei Kassel: Prolog-Verlag, S. 137-161.

Breidenstein, Georg (2006): Teilnahme am Unterricht. Ethnographische Studien zum Schülerjob. Studien zur Schul- und Bildungsforschung. Wiesbaden: VS Verlag für Sozialwissenschaften.

Budde, Jürgen/Heynoldt, Benjamin/Offen, Susanne (2016): Diversität und Inklusion. In: Ottersbach, M./Platte, A./Rosen, L. (Hrsg.): Soziale Ungleichheiten als Herausforderung für inklusive Bildung. Wiesbaden: Springer Fachmedien Wiesbaden, S. 103-130.

Budde, Jürgen/Hummrich, Merle (2013): Reflexive Inklusion. In: Zeitschrift für Inklusion, 4. Onlinezeitschrift ohne Seitenangaben. [http://www.inklusion-online.net/index.php/ inklusion-online/article/view/193/199], Zugriff am 21.11.2016.

Charmaz, Kathy (2006): Constructing grounded theory. A practical guide through qualitative analysis. London, Thousand Oaks, Calif:: Sage Publications.

Cramer, Colin/Harant, Martin (2014): Inklusion - Interdisziplinäre Kritik und Perspektiven von Begriff und Gegenstand. In: Zeitschrift für Erziehungswissenschaft 17, 4, S. 639-659.

Diehm, Isabell/Kuhn, Melanie/Machold, Claudia (2013): Ethnomethodologie und Ungleichheit? Methodologische Herausforderungen einer ethnographischen Differenzforschung. In: Budde, J. (Hrsg.): Unscharfe Einsätze: (Re-)Produktion von Heterogenität im schulischen Feld. Wiesbaden: VS Verlag für Sozialwissenschaften, S. 29-51.

Fritzsche, Bettina (2014): Inklusion als Exklusion. Differenzproduktionen im Rahmen des schulischen Anerkennungsgeschehens. In: Tervooren, A./Engel, N./Göhlich, M./Miethe, 
I./Reh, S. (Hrsg.): Ethnographie und Differenz in pädagogischen Feldern. Internationale Entwicklungen erziehungswissenschaftlicher Forschung. Bielefeld: Transcript-Verlag, S. 329-347.

Hazibar,Kerstin/Mecheril,Paul(2013):EsgibtkeinerichtigePädagogikinfalschengesellschaftlichen Verhältnissen. Widerspruch als Grundkategorie einer Behinderungspädagogik. In: Zeitschrift für Inklusion, 1. Onlinezeitschrift ohne Seitenangaben. [http://www.inklusion-online.net/ index.php/inklusion-online/article/view/23/23], Zugriff am 18.11.2016.

Herzmann, Petra/Merl, Thorsten/Panagiotopoulou, Argyro/Rosen, Lisa/Winter, Julia (i.D.): »Auszeit« vom inklusiven Unterricht. Erste Ergebnisse zu differenzkonstruierenden Praktiken aus ethnographischen Feldstudien in Nordrhein-Westfalen. In: Budde, J./Dlugosch, A./Sturm, T. (Hrsg.): (Re-)Konstruktive Inklusionsforschung. Differenzlinien - Handlungsfelder Empirische Zugänge. Leverkusen \& Berlin: Verlag Barbara Budrich.

Hillebrandt, Frank (2014): Soziologische Praxistheorien. Eine Einführung. Soziologische Theorie. Wiesbaden: VS Verlag für Sozialwissenschaften.

Hinz, Andreas (2015): Inklusion als Vision und Brücken zum Alltag. Über Anliegen, Umformungen und Notwendigkeiten schulischer Inklusion. In: Häcker, T./Walm, M. (Hrsg.): Inklusion als Entwicklung. Konsequenzen für Schule und Lehrerbildung. Bad Heilbrunn: Klinkhardt, S. 68-84.

Kiesel, Doron (1996): Das Dilemma der Differenz: zur Kritik des Kulturalismus in der interkulturellen Pädagogik. Frankfurt am Main: Cooperative-Verlag.

Machold, Claudia (2015): Kinder und Differenz. Eine ethnografische Studie im elementarpädagogischen Kontext. Wiesbaden: VS Verlag für Sozialwissenschaften.

Merl, Thorsten/Winter, Julia (2014): Qualitative Befunde zur Inklusion in der Schule. In: Franz, E.-K./Trumpa, S./Esslinger-Hinz, I. (Hrsg.): Inklusion: Eine Herausforderung für die Grundschulpädagogik. Baltmannsweiler: Schneider Verlag Hohengehren, S. 47-60.

Meseth, Wolfgang/Proske, Matthias/Radtke, Frank-Olaf (2011): Was leistet eine kommunikationstheoretische Modellierung des Gegenstandes „Unterricht“? In: Meseth, W./Proske, M./Radtke, F.-O. (Hrsg.): Unterrichtstheorien in Forschung und Lehre. Bad Heilbrunn: Klinkhardt, S. 223-240.

Panagiotopoulou, Argyro (2013): Ethnographische Zugänge in der frühkindlichen Bildungsforschung. In: Stamm, M./Edelmann, D. (Hrsg.): Handbuch frühkindliche Bildungsforschung. Wiesbaden: Springer, S. 771-786.

Rabenstein, Kerstin/Reh, Sabine/Ricken, Norbert/Idel, Till-Sebastian (2013): Ethnographie pädagogischer Differenzordnungen. Methodologische Probleme einer ethnographischen Erforschung der sozial selektiven Herstellung von Schulerfolg im Unterricht. In: Zeitschrift für Pädagogik 59, 5, S. 668-690.

Rabenstein, Kerstin/Idel, Till-Sebastian/Ricken, Norbert (2015): Zur Verschiebung von Leistung im individualisierten Unterricht. Empirische und theoretische Befunde zur schulischen Leitdifferenz. In: Budde, J./Blasse, N./Bossen, A./Rißler, G. (Hrsg.): Heterogenitätsforschung. Empirische und theoretische Perspektiven. Weinheim u. a.: Beltz, S. 241-258.

Reckwitz, Andreas (2003): Grundelemente einer Theorie sozialer Praktiken. Eine sozialtheoretische Perspektive. In: Zeitschrift für Soziologie 32, 4, S. 282-301.

Reckwitz, Andreas (2008): Praktiken und Diskurse. Eine sozialtheoretische und methodologische Relation. In: Kalthoff, H./Hirschauer, S./Lindemann, G. (Hrsg.): Theoretische Empirie. Zur Relevanz qualitativer Forschung. Frankfurt am Main: Suhrkamp, S. 188-209.

Reh, Sabine (2013): Die Produktion von (Un-)Selbständigkeit in individualisierten Lernformen. Zur Analyse von schulischen Subjektivierungspraktiken. In: Gelhard, A./Alkemeyer, T./ Ricken, N. (Hrsg.): Techniken der Subjektivierung. München: Wilhelm Fink, S. 189-201.

Reh, Sabine/Idel, Till-Sebastian/Rabenstein, Kerstin/Fritzsche, Bettina (2015): Ganztagsschulforschung als Transformationsforschung. Theoretische und empirische Erträge des 
Projekts. In: Reh, S./Fritzsche, B./Idel, T.-S./Rabenstein, K. (Hrsg.): Lernkulturen. Rekonstruktion pädagogischer Praktiken an Ganztagsschulen. Wiesbaden: VS Verlag für Sozialwissenschaften, S. 297-336.

Reh, Sabine/Labede, Julia (2009): Soziale Ordnung im Wochenplanunterricht. In: Boer, H. de/ Deckert-Peaceman, H. (Hrsg.): Kinder in der Schule. Zwischen Gleichaltrigenkultur und schulischer Ordnung. Wiesbaden: VS Verlag für Sozialwissenschaften, S. 159-176.

Reh, Sabine/Rabenstein, Kerstin (2012): Normen der Anerkennbarkeit in pädagogischen Ordnungen. Empirische Explorationen zur Norm der Selbständigkeit. In: Ricken, N./ Balzer, N. (Hrsg.): Judith Butler: Pädagogische Lektüren. Wiesbaden: VS Verlag für Sozialwissenschaften, S. 225-246.

Reh, Sabine/Rabenstein, Kerstin/Idel, Till-Sebastian (2011): Unterricht als pädagogische Ordnung. Eine praxistheoretische Perspektive. In: Meseth, W./Proske, M./Radtke, F.-O. (Hrsg.): Unterrichtstheorien in Forschung und Lehre. Bad Heilbrunn: Klinkhardt, S. 209222.

Reh, Sabine/Ricken, Norbert (2012): Das Konzept der Adressierung. Zur Methodologie einer qualitativ-empirischen Erforschung von Subjektivation. In: Miethe, I./Müller, H.-R. (Hrsg.): Qualitative Bildungsforschung und Bildungstheorie. Opladen, Berlin, Toronto: Verlag Barbara Budrich, S. 35-56.

Ricken, Norbert (2014): Adressierung und(Re-)Signifizierung. Anmerkungen zum Zusammenhang von sozialer Herkunft und schulischer Leistung aus praktikentheoretischer Perspektive. In: Kleiner, B./Rose, N. (Hrsg.): (Re-)Produktion von Ungleichheiten im Schulalltag. Judith Butlers Konzept der Subjektivation in der erziehungswissenschaftlichen Forschung. Opladen u. a.: Verlag Barbara Budrich, S. 119-133.

Schatzki, Theodore R. (1996): Social Practices. A Wittgensteinian Approach to Human Activity and the Social. Cambridge: Cambridge University Press.

Schatzki, Theodore R. (2001): Introduction: practice theory. In: Schatzki, T. R./Knorr-Cetina, K./Savigny, E. v. (Hrsg.): The practice turn in contemporary theory. New York: Routledge, S. 10-23.

Schatzki, Theodore R. (2002): The site of the social: a philosophical account of the constitution of social life and change. Pennsylvania: Pennsylvania State University Press.

Schatzki, Theodore R. (2012): A primer on practices: Theory and research. In: Higgs, J./Barnett, R./Billet, S./Hutchings, M./Trede, F. (Hrsg.): Practice-based education. Perspectives and strategies. Rotterdam, Boston: SensePublishers, S. 13-26.

Schatzki, Theodore R./Knorr-Cetina, Karin/Savigny, Eike von (Hrsg.) (2001): The practice turn in contemporary theory. New York: Routledge.

Spradley, James P. (1979): The ethnographic interview. New York: Holt, Rinehart and Winston.

Stichweh, Rudolf (2013): Inklusion und Exklusion in der Weltgesellschaft - am Beispiel der Schule und des Erziehungssystems. In: Zeitschrift für Inklusion, 1. Onlinezeitschrift ohne Seitenangaben. [http://www.inklusion-online.net/index.php/inklusion-online/article/ view/22/22], Zugriff am 18.11.2016.

Sturm, Tanja (2012): Praxeologische Unterrichtsforschung und ihr Beitrag zu inklusivem Unterricht. In: Zeitschrift für Inklusion, 1-2. Onlinezeitschrift ohne Seitenangaben. [http:// www.inklusion-online.net/index.php/inklusion-online/article/view/65/65], Zugriff am 18.11.2016.

Tenorth, Heinz-Elmar (2013): Inklusion - Prämissen und Problemzonen eines kontroversen Themas. In: Baumert, J./Masuhr, V./Möller, J./Riecke-Baulecke, T./Tenorth, H.-E./Werning, R. (Hrsg.): Inklusion. Forschungsergebnisse und Perspektiven. Schulmanagement -Handbuch, Band 32. München: Oldenbourg Schulbuchverlag, S. 6-14.

Werning, Rolf (2014): Stichwort: Schulische Inklusion. In: Zeitschrift für Erziehungswissenschaft 17, 4, S. 601-623. 


\title{
Deutungsmuster in der Professionsforschung. Anwendung und Ertrag dieses Analyseverfahrens am Beispiel schulischer Sexualerziehung
}

\begin{abstract}
Zusammenfassung
Nach einer einführenden Darstellung bisheriger Überlegungen und exemplarischer Untersuchungen zur Deutungsmusteranalyse in der Professionsforschung wird präzisiert, wann Deutungsmusteranalysen in schul- und unterrichtsbezogenen Kontexten ertragreich erscheinen. Es wird eine terminologische Bestimmung sowie ein idealtypisches Design der Deutungsmusteranalyse vorgeschlagen. Anhand des Unterrichtsthemas „Prostitution“ werden drei Deutungen rekonstruiert. Diese werden in einem weiteren Schritt zu Deutungsmustern abstrahiert. Abschließend folgt eine kritische Einschätzung der Ergebnisse hinsichtlich des Ertrags von Deutungsmusteranalysen für die Professionsforschung.

Schlagwörter: Deutungsmuster, problemzentriertes Interview, Professionalisierung, Prostitution, qualitative Analyse, Sexualerziehung
\end{abstract}

Interpretation patterns in professorial research. Application and yield of this analysis method using the example of sexual education

After an introductory presentation of previous considerations and exemplary researches on the interpretative pattern analysis, it is specified when interpretive pattern analyzes appear to be productive in school and teaching-related contexts. A terminological determination as well as an ideal-type design of the interpretative pattern analysis is proposed. Based on the lesson about "prostitution", three interpretations are reconstructed. In a further step, these are abstracted to interpretative patterns. Finally, a critical assessment of the results regarding the yield of interpretative pattern analyzes for researches on teacher professionalism is given. Keywords: interpretative pattern, problem-centred interview, professionalization, prostitution; qualitative analysis, sexual education

Der Beitrag hat zum Ziel, das Verfahren der Deutungsmusteranalyse am Beispiel des Unterrichtsthemas „Prostitution“ vorzustellen und zu diskutieren. Die zugrundeliegende professionsbezogene Herausforderung besteht darin, dass schulische Sexualerziehung spezifische Handlungsprobleme für Lehrer_innen aufwirft (vgl. Hoffmann 2016). Diese Handlungsprobleme hängen unmittelbar mit dem Unterrichtsgegenstand ,Sexualität zusammen: Eine eigene Wissensbasierung hinsichtlich des Unterrichtsthemas Sexualität erscheint aufgrund fehlender universitärer Professionalisierungsangebote fraglich. Dennoch sind an Lehrer_innen hohe gesellschaftliche Erwartungen gerichtet, den Jugendlichen im Rahmen schulischer Sexualerziehung einen kompetenten Umgang mit sexualbezogenen Aspekten nahezubringen. Wissenschaftlich unbetrachtet bleibt bislang, wie Lehrer_innen die professionsbezogene Handlungsaufforderung bearbeiten, als Lehrer_in mit Schüler_innen über soziale und moralische Aspekte von Sexualität im Unterricht sprechen zu müssen. 
- Unter einer moralischen Perspektive ist das Thema Prostitution problembehaftet, intimisiert und tabu, gesellschaftlich jedoch ist Prostitution ein Phänomen, das für die Jugendlichen sowohl räumlich als auch thematisch präsent ist. Für Lehrende stellt sich die Frage, wie sich ein professioneller Umgang mit einem Thema ausgestaltet, das moralisch negativ bewertet, gleichzeitig faktisch aber etabliert ist.

- Als zweites Problem müssen Lehrpersonen festlegen, mit welchem Ziel dieses auch unter den Jugendlichen womöglich kontrovers gedeutete Thema angesprochen werden sollte. Was sind Lernziele, die mit dem Thema Prostitution einhergehen?

- Drittens ist die spezifische Wissensbasis von Lehrenden unklar: Lehrende sind ebenso wie Schüler_innen mit vielen unterschiedlichen Deutungen über Prostitution und deren Unteraspekte (z. B. zu Gesundheit, zur historischen Entwicklungen, zur rechtlichen Situation usw.) konfrontiert. Eine gemeinsame, von der Profession spezifisch geteilte Expertise ist fraglich. Was Lehrende für Wissensinhalte anbieten, auf welche Wissensgrundlage sie ihre Überlegungen zum Unterrichtsthema Prostitution stützen, ist bislang nicht erforscht.

Vor dem Hintergrund fehlender Forschungsbefunde zu uneindeutigen Themen der schulischen Sexualerziehung im Allgemeinen sowie fehlenden Erkenntnissen zur unterrichtlichen Behandlung des Themas Prostitution im Besonderen sollen Deutungsmusteranalysen als Analyseverfahren vorgeschlagen werden, um grundlegende professionsbezogene Herausforderungen bezogen auf die Handlungsaufforderung „Sprechen über Prostitution“" offenzulegen.

\section{Zum Stand der Diskussion - Forschungstheoretische Einbettung von Deutungsmusteranalysen}

Ein überwiegend geteiltes Verständnis von ,Deutungsmustern“ ist, dass diese als relativ zeitstabile abstrakte Bündelung ähnlicher Interpretationen von strukturell bedingten Problemen und Unklarheiten durch eine abgrenzbare soziale Gruppe auf konkreter, situativ-sozialer Ebene zu fassen sind (vgl. Bennewitz 2008; Bögelein 2016; Kunze 2011). Diese Muster bilden sich sozialisatorisch in alltäglichen Wahrnehmungs- und Interaktionsprozessen aus. Sie bieten als vielfache einzelne Deutungen einen subjektiven Orientierungsrahmen für grundlegende situative und soziale Wahrnehmungen. Die einzelnen Deutungen lassen das Unbekannte jeder neuen sozialen Situation bekannt erscheinen und halten die eigene Handlungsfähigkeit aufrecht, indem sie praktische Lösungen zu überkomplexen oder uneindeutigen Situationen bieten. Als Deutungsmuster verdichtet bilden sie Interpretations- und Handlungsschemata ab, die auf (typische) Strukturprobleme im betrachteten Handlungsfeld verweisen (vgl. Lüders/Meuser 2002).

Dieses überwiegend geteilte Verständnis besteht jedoch nicht seit Beginn der Diskussion um Deutungsmuster; und auch aktuell herrscht bei verschiedenen Forschungsprojekten eine gewisse Uneinheitlichkeit besonders in der grundlegenden Frage, ob als Fallhorizont eine Person (und damit ein einzelnes Interview) oder ein Bezugsproblem (und damit ein thematischer Vergleich mindestens zweier Interviews) verstanden wird. Um diese zentrale Frage des Fallverständnisses zu erklären, werden zunächst wichtige 
Diskussionsbeiträge zu Deutungsmustern seit den 1970er Jahren skizziert; abschließend wird die eigene Verortung dargestellt.

Meist wird Oevermanns „Analyse der Struktur von sozialen Deutungsmustern“ (1973; weiterführend kommentiert 2001) als Beginn einer konzeptuellen Auseinandersetzung benannt. Für ihn gilt es, ,Sinnzusammenhänge und Interpretationsmuster, die dem konkreten Handlungssubjekt als objektive Strukturen gegenübertreten“ (Oevermann 1973: 3), zu rekonstruieren. Unterstellt werden dabei „strukturbedingte Handlungsprobleme" (ebd.: 4), die unabhängig von konkreten Personen existieren, zu denen sich jedoch Akteure verhalten müssen (wie z. B. Lehrer_innen zur Ambivalenz des Unterrichtsthemas Prostitution). Zwar unterlag Oevermanns damaligem Entwurf ein zeitkontextuell strukturalistisches Verständnis von Sozialität (so sprach er von den „Regelhaftigkeiten der ,inneren Logik“ von Erwartungssystemen“, die zu einer ,,konsistenten Interpretation und Deutung “ führen; vgl. ebd.; Herv. d.V.); aber auf individueller Ebene entstünden ,komplexe Einstellungsmuster wesentlich auch durch vom Individuum selbsttätig geleistetes ,Ausbuchstabieren' von Implikationen weniger zentraler ,Schlüsselkonzepte“"“ (ebd.: 17f.; Herv. d.V.).

Zehn Jahre nach Oevermanns Diskussionsanstoß fokussierte Arnold (1983) in einer vielbeachteten methodologischen Auseinandersetzung besonders die sozialisatorische Aneignung von Deutungsmustern. Diese gelten nicht nur für den individuellen Einzelfall, sondern seien vergleichbar für größere soziale Einheiten. Mit Rückbezug auf den dialektischen Streit zwischen Strukturalismus und Sozialkonstruktivismus schlussfolgert er, dass die „Ermittlung und Explikation von Deutungsmustern [...] auf den Nachvollzug der Prozesse der Bedeutungsverleihung aus der Perspektive der handelnden Subjekte“ (ebd.: 895; Herv. d.V.) zu beziehen sei. Für die heutige Diskussion bedeutsam ist Arnolds handlungspraktische Ableitung, dass Deutungsmuster in Form einzelner Deutungen ,unmittelbares, quasiroutiniertes Handeln auch und gerade unter Zeitdruck sowie angesichts problematischer Situationen“ (ebd.) ermöglichen.

In der weitergeführten Diskussion stellte sich ein überwiegend geteiltes Verständnis von Deutungsmusteranalysen heraus, das besonders von Ullrich (1999) beschrieben wurde. Das hermeneutisch oft genutzte Vorgehen der Einzelfallrekonstruktion stellt sich für Ullrich als nicht zweckdienlich heraus, wenn zwischen den einzelnen Individuen übergreifende „Bezugsprobleme“ (ebd.: 443) von Forschungsinteresse sind. Was als Bezugsproblem wahrgenommen werde, hänge ,zum einen von den sozial verfügbaren Deutungsmustern ab; andererseits üben die strukturellen Gegebenheiten aber auch objektive und insofern interpretationsunabhängige Zwänge auf die Akteure aus" (ebd.: 430). Ein zentrales Moment von Deutungsmusteranalysen stellt sich für Ullrich in der Loslösung von Einzelfallauswertungen dar. Das Argument gegen eine Einzelfallauswertung sei, dass ,nur durch den Vergleich und das ,Übereinanderlegen““ (ebd.: 443; Herv. d.V.) soziale Deutungsmuster identifizierbar und rekonstruierbar seien. Dieses Lösen vom Einzelfallverständnis bezogen auf alle Aussagen einer interviewten Person bestimmt Ullrich als hermeneutische Stärke von Deutungsmusteranalysen. Dabei wendet er sich gegen die von Oevermann stets mit der Deutungsmusteranalyse verknüpfte Objektive Hermeneutik mit der Begründung, dass einerseits mit dem allem zugrundeliegenden Strukturbegriff ,kein theoretisches oder methodisches Mittel zur sozialen Lokalisierung von Deutungsmustern zur Verfügung“ (ebd.: 432) stehe; andererseits 
dürfte die „Rekonstruktion mehrerer, hinsichtlich eines Bezugsproblems konkurrierender Deutungsmuster mit sequentiellen Einzelfallanalysen allein kaum gelingen“ (ebd; Herv. d.V.).

Die hier grundlegende Positionierung des später aufgezeigten empirischen Materials entspricht dem Fallverständnis im Sinne eines Bezugsproblems: Ein für alle Lehrer innen zu bearbeitendes Problem konnte durch den Vergleich mehrerer Interviews identifiziert und rekonstruiert werden. Ein „Fall“ stellt damit nicht jede sinnlogische Sequenz eines einzelnen Interviews dar, sondern die thematische Bündelung unterschiedlicher Sequenzen aus dem gesamten empirischen Material, also allen Interviews, die sich auf das gemeinsame Bezugsproblem richten.

\section{Deutungsmuster als Analyseverfahren für Schul- und Unterrichtsforschungen}

In der Schul- und Unterrichtsforschung bewegt sich der Fallhorizont ebenso zwischen einem Fallverständnis auf Einzelpersonenebene oder einem Fallverständnis i. S. eines interpersonal identifizierten Bezugsproblems.

Kunze (2011) beispielsweise nutzt bei ihren Fragen nach der „Professionalisierung als biographischem Projekt" Deutungsmusteranalysen, um durch den systematischen Vergleich zweier ausgewählter Einzelfallrekonstruktionen Unterschiede der ,grundsätzlichen Haltung bzw. den allgemeinen Zugang zum Beruf“" (vgl. ebd.: 325) zu rekonstruieren. Durch das personale Fall-Verständnis sowie die biographisch-rekonstruktive Erarbeitung werden die Fall-Kontrastierungen vor allem durch intensive induktive Vergleiche zweier Einzel-Interviews generiert. Eine noch deutlichere Orientierung am Fall als Aussagen einer Person lässt sich bei Meister auffinden, die zum „unterrichtlichen Selbstverständnis von LehrerInnen“ Deutungsmuster analysiert. Durch die ausschließliche Orientierung an Oevermanns Deutungsmusterkonzeption sowie die sehr stark biografisch orientierten Einzelfallrekonstruktionen und das induktive Vorgehen endet die empirische Analyse mit einer Rekonstruktion von vier Biografien verstanden als vier Einzelfälle, die Meister als „Muster“ bezeichnet. Weigelt (2008) legt ein Verständnis eines Falls i. S. einer Bearbeitung struktureller professionsbezogener Handlungsprobleme vor. Mit dieser Herangehensweise löst sie die Sequenzen in einem Interview dahingehend auf, dass ein Interview mehrere „Fälle“ beinhalten kann und ein (am Bezugsproblem orientierter) Fall aus Sequenzen unterschiedlicher Interviews besteht. Damit ist für Weigelt nicht die einzelne Person das Fallkriterium, sondern das verbindende Bezugsproblem und deren unterschiedliche Bearbeitungen.

Eine Gemeinsamkeit der Forschungen besteht darin, dass bei einem bestimmten Arrangement von Daten und Theorien Deutungsmusteranalysen gewinnbringend erscheinen. Deutungsmusteranalysen bieten sich besonders an, wenn es (1) über das Forschungsfeld nur wenige Konzepte gibt, die theoretisch-abstrakt vorliegen und keine Ableitung für konkrete Handlungen beinhalten. Diese sogenannten „sensitizing concepts“ (Blumer 1954: 7) sind nicht ohne weiteres in einem Interview expliziert und müssen erst durch Interpretationen identifiziert werden (wie z. B. die professionstheoretische „Nähe-Distanz-Antinomie“). Mit Deutungsmusteranalysen kann rekonstruiert werden, 
wie sich auf der Handlungs- bzw. Erzählebene eine abstrakte Theorie ausgestaltet und wie sie durch die Akteure mit konkreten Inhalten gefüllt wird. Deutungsmusteranalysen eignen sich ebenso, wenn das Forschungsfeld (2) dahingehend unbestimmt ist, dass Unbekanntes in den empirischen Daten grundsätzlich erarbeitet und theoretisiert werden kann. Als letzte Tendenz aktueller Deutungsmusteranalysen lässt sich feststellen, dass (3) nicht alle Aspekte jedes Interviews schlussendlich eine theoretische Modellierung erfahren und damit ein Fall einem Interview gleichkommt. Der Gewinn von Deutungsmusteranalysen ist es, zwischen bereits gut beforschten Sachverhalten bzw. nicht forschungszentralen Aussagen und unbekannten Elementen bzw. forschungsnahen Aspekten zu unterscheiden und fall-übergreifende Bezugsprobleme und deren Modi der Bearbeitung zu rekonstruieren.

\section{Methodologische Offenlegung: Deutungsmusteranalysen als Rekonstruktion von professionsbezogenen Handlungsproblemen am Beispiel „Sprechen über Prostitution“ 1}

Die Interviews wurden mit Lehrpersonen geführt, die in der Sekundarstufe I in den Fächern Biologie bzw. Naturwissenschaften, Religion bzw. entsprechender Ersatzfächer sowie Gesellschaftslehre/Sozialkunde bereits die Unterrichtsreihe Sexualerziehung selbst durchgeführt hatten. Als qualitative Interviewform wurde das Problemzentrierte Interview (Witzel 1989; Witzel/Reiter 2012) wie folgt weiterentwickelt:

1 Erzählungsgenerierende Kommunikationsphase

1a vorformulierte Einleitungsfrage

$1 \mathrm{~b}$ allgemeine Sondierung

2 Verständnisgenerierende Kommunikationsphase

2a spezifische Sondierungen

$2 b$ ad-hoc-Fragen

Zunächst soll in der (1) erzählungsgenerierenden Kommunikationsphase der Interviewte zu möglichst langen Erzählungen über den Gegenstand animiert werden. Dabei ist (a) die vorformulierte, offene Einleitungsfrage das Mittel, um das Gespräch direkt auf das beforschte Problem zu lenken. Nach ersten narrativen Erzählungen dienen (b) allgemeine Sondierungen dazu, die subjektiven Problemsichten der Interviewten vertiefend offenzulegen. In der zweiten Interviewphase werden (2) verständnisgenerierende Kommunikationsstrategien angewandt, bei denen der Interviewer nun Themen vorgibt, alternative Perspektiven einbringt oder Deutungen des Interviewten kritisch widerspiegelt. Dabei kommen (a) spezifische Sondierungen zum Tragen, in denen aus Theorie und Empirie abgeleitete Fragen gestellt werden. Ebenfalls wird in der zweiten Interviewphase

1 Die dargestellten methodologischen und methodischen Konzeptionen und Ausführungen entstammen einem qualitativen Forschungsprojekt zur schulischen Sexualerziehung und sind ausführlich in Hoffmann (2016) beschrieben. 
mit (b) ad-hoc-Fragen gearbeitet; also Fragen, deren Notwendigkeit sich ergibt, wenn bestimmte Themenbereiche nicht angesprochen wurden, die jedoch bei der Deutungsmusteranalyse aufgrund der Vergleichbarkeit der Deutungen eingeholt werden sollen.

Die Datenaufbereitung und Analyse geschah maßgeblich gemäß den methodologischen Überlegungen von Kelle/Kluge (2010). Bei der Datenaufbereitung wurde das transkribierte Material zunächst kodiert (vgl. ebd.: 56ff.). Dabei wurden induktive Kodierungen entlang des gesamten Materials gebildet. Gleichzeitig dienten abstrakte Konzepte als Heuristik für deduktive Kodierungen wie beispielsweise „Nähe-Distanz-Ambivalenz". Nach der Kodierung des Materials geschah die Bildung von Subkategorien und deren Dimensionalisierung (also vergleichende Anordnung nach inhaltlichen Kriterien). Das Ziel dabei war es, Kategorien zu identifizieren, deren Subkategorien möglichst unterscheidbar zu anderen Fällen sind, um eine möglichst hohe Varianz an Ausprägungen jeder Subkategorie aus dem gesamten Material zu generieren. Die dafür angewendete Fallkontrastierung in Form des „Qualitativen Sampling“ (vgl. ebd.: 41ff.) geschah sowohl deduktiv mithilfe der theoriegeleiteten Identifikation von Gegenbeispielen als auch induktiv nach der Maßgabe minimaler und maximaler Kontrastierung. Diese erarbeiteten Kategoriensysteme stellten jeweils Deutungen zu spezifischen Sachverhalten dar, die nun vergleichend angeordnet und in einem letzten Schritt zu empirisch begründeten Deutungsmustern, verweisend auf ein Bezugsproblem, aggregiert wurden.

Hinsichtlich der Begrifflichkeit erfolgt nun eine präzise Bestimmung, um das Vorgehen besser nachvollziehbar zu machen:

- Als Bezugsproblem soll ein mit der Unterrichtsreihe einhergehendes theoretisches Professionsproblem verstanden werden. Bezugsprobleme sind analytisch-abstrakte, vom Interpreten identifizierte Handlungsanforderungen des Unterrichts, auf deren Bewältigung Deutungen in unterschiedlichen Fällen verweisen.

- Deutungen beziehen sich auf die in den Daten vorliegenden konkreten Schilderungen, Behauptungen, Aussagen usw. der interviewten Personen. In Deutungen kommunizierte Aussagen stellen sich als fall-immanente Interpretationen, Lösungen und Perspektiven konkreter Situationen dar.

- Deutungsmuster sind die vom einzelnen Interview und der konkreten Narration abstrahierten Modi der Bearbeitung des Bezugsproblems. Sie sind ebenfalls analytische Kategorien, die im Rahmen der Datenauswertung generiert werden. Sie werden durch den Vergleich von unterschiedlichen Deutungen zum identischen Bezugsproblem herausgearbeitet und voneinander abgegrenzt. Deutungsmuster verweisen auf einen grundsätzlichen Umgang mit dem Bezugsproblem.

Die methodologischen Überlegungen lassen sich schematisch wie folgt darstellen: 


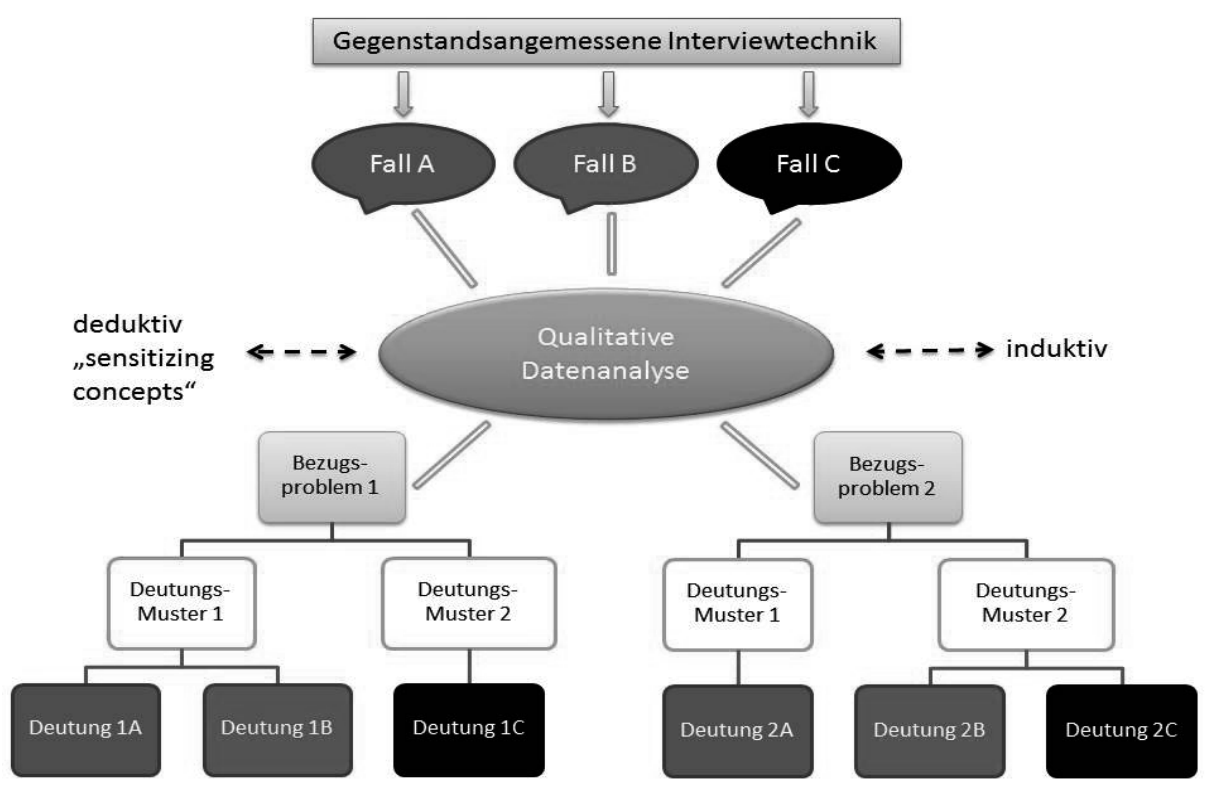

Abbildung 1: Schematische Darstellung Deutungsmusteranalyse

Unterschiedliche induktiv und deduktiv identifizierte schul- und unterrichtsbezogene Probleme (Bezugsproblem 1, 2, ...), die mit professionellen Handlungsaufforderungen einhergehen, werden von Lehrer_innen unterschiedlich interpretiert (Deutungsmuster 1, $2, \ldots)$. Den Deutungsmustern liegen problembezogene Deutungen in unterschiedlichen Fällen (A, B, ...) zugrunde, die auf ein identisches Bezugsproblem verweisen (1 A, 2A, 3A usw.).

\section{Darstellung der Deutungsmuster zu „Prostitution“}

Der Analysegegenstand „Prostitution als mögliches Unterrichtsthema“ birgt eine große Komplexität, da unterschiedliche Argumente zu einzelnen Facetten von Prostitution in Konkurrenz zueinander stehen. Prostitution wird tendenziell selten im Unterricht angesprochen, gleichzeitig von den Jugendlichen allerdings als Thema gewünscht (vgl. BZgA 2010: 42). Das wirft die Frage auf, warum Lehrende ein Themengebiet, das Schüler_innen interessiert und damit hinsichtlich der Lernmotivation besonders ergiebig zu sein scheint, für den Unterricht nicht nutzen. Eine qualitative Rekonstruktion zweier Deutungen und deren Verdichtung zu einem Deutungsmuster zum Unterrichtsthema Prostitution streicht Argumente von Lehrpersonen heraus, die zur Nicht-Behandlung des Themas führen. Außerdem wird ein alternatives Muster vorgestellt, bei dem Prostitution zum Unterrichtsthema gemacht wird. 


\subsection{Deutung: persönliche Erfahrungslosigkeit}

Die folgende Aussage äußert der Lehrer Harald in der ersten, erzählungsgenerierenden Interviewphase. Der Interviewer fragt noch nicht nach konkreten Inhalten, sondern holt allgemeine Erzählungen und Deutungen ein.

Harald: /Ähm/ (2.0) es kommt immer so ,n bisschen /äh/ganz automatisch durch die Fragestunden mit 'rein: Prostitution.

Int: Mhm.

Harald: Und /äh/ich denke, auch das sollte man irgendwo /äh/ thematisieren, /ähm/ wobei manche Jungs das irgendwo so als selbstverständlich hinnehmen. Ja? ,Ja, wo soll man denn hingehen, wenn man keine Freundin hat?' Und dergleichen, /äh/ während Mädchen das dann /äh/ wesentlich kritischer sehen. Und /äh/ das ist sicherlich 'n schwieriges Thema. (83-89)

Zunächst nimmt der Interviewte wahr, dass Jugendliche am Thema Prostitution starkes Interesse zeigen. Das Thema käme durch Fragen der Jugendlichen ,immer [...] ganz automatisch [...] mit rein“. Mit dem Bild eines Automatismus verweist Harald auf einen schematischen Ablauf, dass Fragestunden seitens der Schüler ,immer“ zum Ansprechen des Themas Prostitution führen. Auffallend ist Haralds vages Sprechen über den Gegenstand, sein Verständnis bleibt durch das mehrfach genannte, unspezifisch geäußerte „das“ vage. Er verweist damit auf ein Problem des Sprechens über Prostitution als Unterrichtsthema: Wie kann und darf über Prostitution gesprochen werden? In welchem Modus findet eine professionelle Kommunikation über Prostitution statt? Fehlende oder unpassende Worte drücken das Problem der kommunikativen Tabuisierung von Sexualität aus. Mit dem unspezifischen ,das“ verweist Harald auf das Problem der Unklarheit und des Heiklen im Sprechen über Sexualität.

Eingebettet in seine auf etwas Unpräzises verweisende Deutung, dass „,manche Jungs das [...] als selbstverständlich“, „Mädchen“ im Gegensatz ,das [...] wesentlich kritischer" betrachten, nimmt Harald eine generelle Bewertung des Themas vor: Prostitution als Thema sei ,sicherlich [...] schwierig“. Durch seine auf Geschlechterdualismen rekurrierende Wahrnehmung verweist Harald auf das grundlegende Problem, dass Prostitution Gegenstand unterschiedlicher Auffassungen und Zuschreibungen ist. Von Bedeutung ist nun Haralds Konstruktion von ,Anderen“. Durch sein Bild von ,,manchen Jungs" verweist er in seinem Sprechen auf Andere, auf Dritte, über die gesprochen wird. Dies ist hinsichtlich des moralischen Sprechens über Prostitution aussagekräftig: Weder involviert Harald sich selbst im Sprechen über das Thema noch generalisiert er und benutzt das abstrakt bleibende „man“, sondern er weist von sich fort auf jene, die eine bestimmte, andere Deutung von Prostitution haben. Durch seinen Geschlechter-Vergleich des einerseits ,irgendwo so als selbstverständlich“ der Jungen, andererseits seiner Wahrnehmung einer kritischeren Einstellung der Mädchen bewertet Harald normativ und moralisierend gleichzeitig: Das Laissez-faire der Jungen wird im Vergleich zu den Sichtweisen der Mädchen als für Harald problematischere Seite rekonstruierbar.

$\mathrm{Zu}$ klären ist, welche Lösung Harald bezüglich des immer wiederkehrenden Schülerwunschs nach Prostitution als Unterrichtsthema anwendet, wenn er das Thema als „schwierig“ würdigt. Eine weitere Passage präzisiert seinen Umgang mit der GesprächsAufforderung: 
Und /äh/, aber die, die Fragen waren dann auch einfach wirklich SPANNEND, /äh/ so Sachen wie: ,Wie sieht es im Puff aus?‘ Ne? Was sagt man als Lehrer dazu? Ich konnte ihnen dann nur sagen: ,Tut mir leid, bin noch nie da gewesen. Ich kann ,s euch nicht sagen.' Ne? ,Ich kenn“ das höchstens aus irgendwelchen Fernsehfilmen oder so, wo sowas dargestellt wird. Aber OB das wirklich die Realität ist, /äh/ kann ich auch nicht sagen.' (25)

Bei der Beantwortung der von Harald als „,spannend“ empfundenen Schülerfragen verweist er zunächst auf seine professionelle Funktion als Lehrer: „Was sagt man als Lehrer dazu?" Harald deutet damit an, dass er professionsbezogene Lösungen sucht, die als generalisierbare Antwortmöglichkeiten für Lehrende gelten können. Er fragt im Interview nicht, was er als Person dazu sagen sollte, sondern zielt auf die institutionelle Rolle, was „man als Lehrer“ antworten könne. Im weiteren Verlauf jedoch verlässt Harald im Umgang mit der Schülerfrage ein professionsbezogenes Antwortverhalten und rekurriert auf sich selbst als Privatperson: Er entschuldigt sich und benennt, dass er ,noch nie dagewesen“ sei und er zu den Schülerfragen daher ,nicht[s] sagen“ könne. Auch könne er nicht sagen, ob das mediale Bild von Bordellen der Realität entspreche.

Haralds Aussagen können zu einer Deutung verdichtet werden: Als konkrete Handlungsaufforderung wird der Schülerwunsch nach Prostitution als Unterrichtsthema benannt. Auf einer diskursiven Ebene deutet Harald einen Unterschied im Verständnis von Prostitution zwischen den Geschlechtern an. Harald verweist dabei aus einer bestimmten, nämlich seiner eigenen moralischen Position auf andere, wobei Jungen durch diese Konstruktion der ,Anderen“ als moralisch unangemessenere Seite identifiziert werden. Durch das selbstpostulierte Unwissen eines allgemeinen professionellen Umgangs als Lehrer mit diesem Thema wird von der Rolle des Lehrers abgesehen und als Person, d. h. unter Rückgriff auf individuelle biografische Erfahrungen, geantwortet. Da er bei Prostitution jedoch eine fehlende biografische Erfahrung äußert, wird Prostitution als Unterrichtsthema dergestalt bearbeitet, dass Harald zwar den grundsätzlichen Wunsch der Schüler_innen aufnimmt, jedoch keine unterrichtlichen Informationen einbringt und dem Schüler_innenwunsch, über Prostitution zu sprechen, mit Negationen begegnet.

\subsection{Deutung: Involvierung der Schüler_innen}

Eine zweite Deutung zum Nicht-Behandeln von Prostitution kann durch Aussagen der Lehrerin Vera herausgearbeitet werden. Vera führt die Unterrichtsreihe Sexualerziehung sowohl ko- als auch monoedukativ durch. Im monoedukativen Part unterrichtet sie die Schülerinnen, die Schüler werden parallel von einem Lehrer unterrichtet. Vera kommt in der ersten Phase des Interviews nicht auf Prostitution zu sprechen und wird in der zweiten Phase durch eine ad-hoc-Frage zur Widergabe ihrer diesbezüglichen Deutung aufgefordert.

„Int: Es gibt noch so 'n artverwandtes Thema: Prostitution. Machst du das irgendwie? Oder kommt das zur Sprache, spielt das 'ne Rolle?

Vera: Nee. [...] Aber /ähm/ (4.0) es ist auch so 'n Thema, was, glaub“ ich, dann sehr heikel ist, weil ja die schon auch die Frauen, also die Mütter wechselnde Partnerschaften haben. Viele Kinder sind von unterschiedlichen Vätern. Und die Beleidigung ,Hurensohn', da gehen sie ja sehr, sehr, sehr hoch. Also so dieses, das wollen sie gar nicht. Das ist (4.0) 
Int: Ja?

Vera: Das ist für die 'ne ziemlich, ziemliche /ähm/ Beleidigung.

Int: Okay.

Vera: Also da reagieren sie auch sehr empfindlich, wenn ihre Mutter, ihre eigene Mutter beleidigt wird. Durchgängig. Und das Thema wird dann auch ausgegliedert, weil ich glaube, sie müssten sich mit 'ner, auch mit 'ner Tatsache ausein/, auseinanderspre/, also auseinandersetzen.“ (273-280)

Nach der sofortigen Verneinung der Frage, ob sie das Thema Prostitution im Unterricht behandelt, wertet Vera das Thema als „,sehr heikel“. Mit ihrer Äußerung „,weil““ begründet sie ihre Einordnung: Das Heikle resultiere aus dem realen Bezug, mit dem Schüler_innen diesem Thema begegneten. Ihre Argumentation fokussiert die Mütter der Schüler_innen: Diese hätten „wechselnde Partnerschaften“, die Kinder seien „von unterschiedlichen Vätern“. In diesem Kontext ordnet sie ein Sprechen über Prostitution als Möglichkeit ein, dass die Schüler_innen ihren eigenen familiären Hintergrund mit dem Thema assoziieren und dies als konkrete Anspielung auf den Lebensstil der Mutter verstehen. Als Begründungsfigur führt Vera an, dass „Hurensohn“ für die Jugendlichen eine sehr schwerwiegende Beleidigung darstelle. Vera spezifiziert ihre Deutung, da die Beleidigung auf die ,eigenen“ Mütter der Jugendlichen abziele. Sie betont in der Interviewsituation durch eine verstärkende Wiederholung ,ihre Mütter, ihre eigenen Mütter“ (Herv. d.V.) die in ihrer Deutung mögliche direkte Involvierung der Jugendlichen mit dem Unterrichtsthema, und dies für die Jugendlichen „durchgängig“. Daran anschließend teilt sie mit, dass dieses Thema in ihrem Unterricht folglich ,,ausgegliedert“ werde, da die Jugendlichen sich andernfalls mit jener „Tatsache [...] auseinandersetzen“ müssten. Als Unterrichtskonsequenz entscheidet sich Vera gegen das Thema, da in ihrer Deutung auf diese Weise den Jugendlichen eine falsche Interpretation der eigenen elterlichen Situation erspart bleibe. Sie sieht kein Ziel, keinen Sinn im Unterrichtsgespräch über Prostitution. Im Gegenteil: In ihrer Deutung erachtet sie das Auslassen des Themas aufgrund der Brisanz, die im Milieu der Jugendlichen existiere, als eher zielführend.

Aus Veras Aussagen lässt sich folgende Deutung generieren: Prostitution taucht als Unterrichtsthema nicht auf. Als Begründung wird herangezogen, dass eine mögliche Fehl-Interpretation der Jugendlichen aufgrund des mütterlichen Verhaltens zu dem von Prostituierten gegeben sei. Dies führe bei einer unterrichtlichen Behandlung auf Seiten der Schüler_innen zu einer Auseinandersetzung mit der eigenen familiären Situation und der Möglichkeit, dass die Schüler_innen falsche Schlussfolgerungen ziehen könnten. Weil dies als heikel angenommen wird, meidet Vera das Thema. Damit markiert der Fall das intentionale Auslassen des Themas aufgrund einer angenommenen Brisanz und möglichen biografischen Fehlinterpretationen auf Seiten der Jugendlichen.

\subsection{Deutung: fragend-entwickelnde Offenheit}

Neben den beiden vorherigen Deutungen, die jeweils durch persönliche Involvierung (entweder des Lehrers oder vermeintlich der Jugendlichen) zur Nicht-Behandlung des Themas im Unterricht führen, soll mit Stefan ein Fall dargestellt werden, in dem Prostitution zum Unterrichtsthema gemacht wird. Stefan unterrichtet das Thema ausschließlich monoedukativ, wobei er - komplementär zu Vera und den weiblichen Schülerinnen - mit den männlichen Schülern alleine ist. Die Grundlage seiner Äußerungen zu Pro- 
stitution ist eine im Interview vorgelegte Schulbuchseite, auf der Prostitution unter der Überschrift „Probleme der Sexualität“ gelistet wird. ${ }^{2}$

ja, also auch die Prostitution sozusagen als ein Problem der Sexualität darzustellen finde ich eben SCHWIERIG. Ja? Also ich ähm natürlich würde ich jetzt mit meinen Schülern darauf eingehen OKAY ähm warum gehen wir denn in den Puff? Ja? Also warum gehen eigentlich Männer in den PUFF. So, äh, das würd ich mit denen natürlich besprechen. (46)

Stefan beginnt mit der Aussage, er fände es „schwierig“, Prostitution als problematisch darzustellen und beschreibt, dass er ,natürlich“ mit den Schülern über die Funktion von Prostitution sprechen würde (,warum“). Seine Deutung lässt an dieser Stelle eine Kontrastierung zu Harald und Vera zu: Stefan benennt eine Offenheit gegenüber der Thematik und stützt seine Aussagen zu Prostitution nicht auf unmittelbare biografische Involvierungen. Von Interesse ist seine zunächst geäußerte Vergemeinschaftung mit den männlichen Schülern (,warum gehen wir"), um diese direkt in einer nachfolgenden Wiederholung auf die männliche Geschlechtsgruppe insgesamt zu verallgemeinern (,warum gehen Männer"). Stefan grenzt sich (im Kontrast zu Harald) nicht von anderen $\mathrm{ab}$, sondern nutzt in diesem Kontext die Figur der Kollektivierung. Dies kann er aufgrund des geschlechtshomogenen Unterrichtssettings machen, ohne dabei Schülerinnen kommunikativ auszuschließen. Durch das Weglassen einer Konstruktion von Dritten, von ,Denen', die Prostitution nutzen, normalisiert er das Sprechen über Prostitution. Stefan betont in seiner Ausweitung auf „Männer“ die sozialstrukturelle Ebene, die gesellschaftliche Einbettung von Prostitution. Dies stützend kann eine weitere Sequenz seines Sprechens über die unterrichtliche Behandlung des Themas aufgegriffen werden:

Also so da würd ich vielmehr die Rolle der Frau, ne, wie soll eigentlich die Frau was für ne Rolle hat sie eigentlich. Ist sie wirklich nur das nackte Luder, oder nimmt sie ne ganz andere Rolle ein. Das kann man da wirklich auch schön mit denen machen. (62)

Stefan verweist explizit auf Mehrperspektivität, mit der das Thema Prostitution betrachtet werden kann, und damit auch auf mögliche unterschiedliche Positionierungen, die gegenüber dem Thema eingenommen werden können. Anders als Harald und Vera rekurriert er damit auf sozialwissenschaftliches Wissen, das für das Unterrichtsgespräch aktiviert werden soll. Im Verweis auf die „Rolle“ der Frau betont er, dass es nicht die Rolle gebe, sondern bei Prostitution auch andere Rollen als die des „nackten Luders“ beachtet werden müssen. Die Ansprache von „Rollen“ stützt die Rekonstruktion eines sozialwissenschaftlichen Fokus', da Rollen i.d.R. in einem funktionalen Zusammenhang zu Institutionen oder sozialen Gruppen stehen. Stefan ordnet dies hinsichtlich eines Gesprächspotentials ein: „Das kann man da wirklich auch schön mit denen machen.“ „Schön“ ist kontextuell als für das Unterrichtsgespräch gut nutzbar zu dechiffrieren; aus Stefans Perspektive heraus bietet das Thema Prostitution gute Möglichkeiten, differen-

2 Der Konjunktiv wird innerhalb der Interviewsequenz aufgelöst, wenn Stefan über konkrete Unterrichtsinhalte spricht, die er unter dem Thema „Prostitution“ subsummiert hat (vgl. als erste Änderung Interview-Absatz 62 weiter unten sowie Hoffmann 2016). Für die hier erfolgte Rekonstruktion ist jedoch der Beginn, die generelle Einordnung des Themas als mögliches Unterrichtsgespräch relevant. 
zierte Betrachtungen im Unterrichtsgespräch herauszuarbeiten. Bestärkt wird die gute Eignung für den eigenen Unterricht durch die verstärkende Bestätigung, er könne das „,wirklich schön“ im Unterricht machen. Durch Stefans Ansprache sozialwissenschaftlichen Wissens entpersonalisiert er das Thema und macht es kommunikativ besprechbar.

\section{Vergleichende Darstellung der Deutungsmuster und Ertrag für die Professionsforschung}

Bei der Behandlung des Themas Prostitution müssen die eingangs erläuterten Probleme der Unklarheit des Sprechen-Über (,wie“), die Frage nach legitimen Zielen des Unterrichtsgesprächs (,wozu“) sowie die Frage nach den professionellen Wissensvorräten, auf die rekurriert wird (,was") von Lehrpersonen bearbeitet werden. Ausgehend davon wurden durch qualitative Analysen drei unterschiedliche Deutungen dargestellt, mit denen Lehrpersonen die Handlungsaufforderung „Prostitution als Unterrichtsthema“ bearbeiten. Hinsichtlich einer Rekonstruktion von Deutungsmustern, verstanden als generalisierte Lösungen von professionsbezogenen Handlungsproblemen, können die Deutungen zu zwei grundsätzlichen Deutungsmustern systematisiert werden, die sich in ein biografisch orientiertes und ein kognitiv-beschreibendes unterteilen:

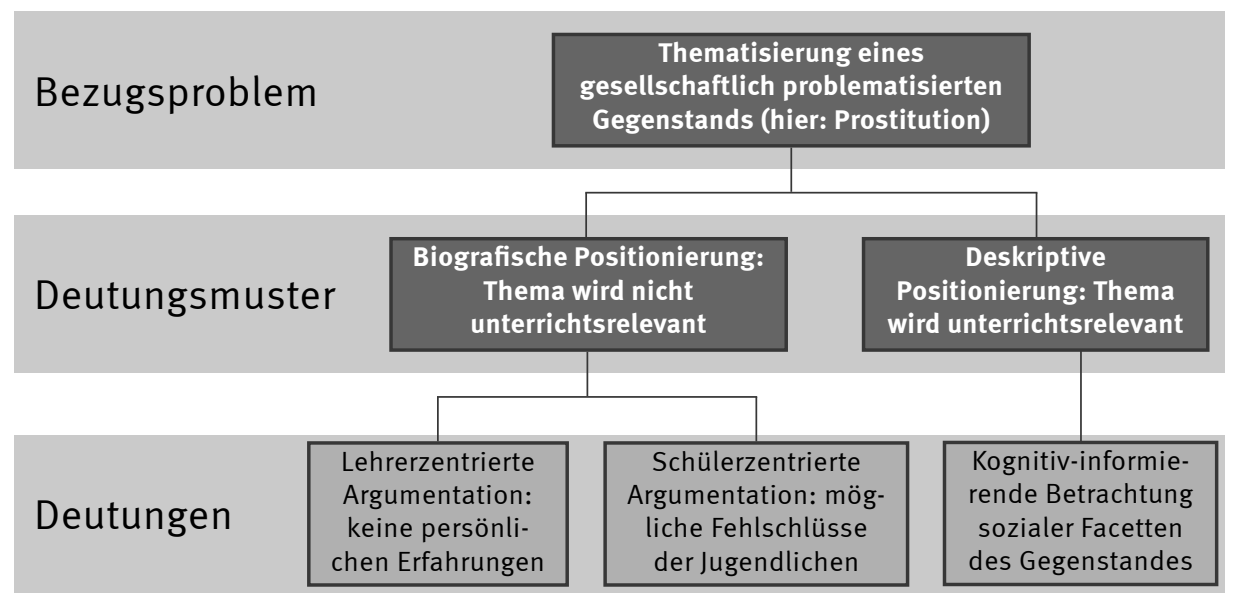

Abbildung 2: Darstellung des Bezugsproblems, Thematisierung gesellschaftlich problematisierter Themen“

In den ersten beiden Deutungen geben die Lehrpersonen an, im Unterricht nicht ausführlich über das Thema Prostitution sprechen zu können. Damit entspricht ihr Vorgehen dem Gros der Lehrkräfte im sexualerzieherischen Unterricht, das Schüler_innen keine kognitiv-informativen Wissensbausteine zu problematisierten Themen der Sexualität anbietet (vgl. BZgA 2010: 42). In den Deutungen lassen sich inhaltliche Gründe und damit erste Erklärungen für eine Nicht-Behandlung rekonstruieren: In der ersten 
Deutung wird konstatiert, dass Prostitution als Unterrichtsgegenstand „ein schwieriges Thema“ sei. Durch den Bezug auf die moralische Mehrdeutigkeit des Gegenstands ist für die Lehrperson unklar, wie sie das Thema professionell gestalten soll. Auf die reale Handlungsaufforderung, mit dem immer wiederkehrenden Schülerwunsch umgehen zu müssen, weiß die Lehrperson unter professionsbezogenen Gesichtspunkten keinen adäquaten Umgang und löst das Handlungsproblem durch Rückgriff auf eigene biografische Erfahrungen: Da die Lehrperson niemals persönlich ein Bordell besucht habe, könne auch zu Prostitution nichts gesagt werden. In der zweiten Deutung ist die leitende Argumentation die Befürchtung, bei den Jugendlichen eine möglicherweise falsche Reflexion über die persönliche Lebensgestaltung der Mütter zu initiieren. Dies führt zu einer Einordnung des Unterrichtsthemas als „,sehr heikel“. In dieser Deutung müssten sich bei Behandlung des Themas Prostitution die Schüler_innen mit eigenen familialen „Tatsachen“ auseinandersetzen. Diese persönlich-biografisch unangemessene Reflexion wird von der Lehrperson angenommen, da die thematisch anschlussfähige Beleidigung (,Hurensohn“) von den Jugendlichen sehr negativ aufgenommen werden würde. In dieser zweiten Deutung wird das Handlungsproblem mit Blick auf die Schüler_innen durch ein konsequentes Auslassen einer unterrichtlichen Bearbeitung von Prostitution gelöst.

Als Kontrast dazu wird in einer dritten Deutung das Thema als unterrichtsgeeignet eingeordnet. Ein biografisch orientierter Zugriff auf im Unterricht anwesende Personen wird von der Lehrperson als ,schwierig“ markiert. Für die Lehrperson liegt die Tauglichkeit gerade im kognitiven Zugang, der dieses Thema für das Unterrichtsgespräch interessant werden lässt. Unter Meidung einer auf Dritte verweisenden Darstellung wird das Sprechen über Prostitution normalisiert. Hinsichtlich des gegenstandsbezogenen Facettenreichtums wird das Thema als unterrichtsgeeignet eingeordnet. Dabei wird eine deskriptive Darstellung des Gegenstands hervorgehoben, bei welcher vor allem kognitive und informative Aspekte der Sexualerziehung angesprochen werden können. Durch eine verallgemeinernde Kommunikationsform wird den Jugendlichen ein einfacherer Zugang zum Sprechen über Sexualität ermöglicht, da Jugendliche ihre diesbezüglichen Gedanken aufgrund der Öffentlichkeit und der Beobachtung durch Peers im Modus von Verallgemeinerungen und Abstraktionen formulieren können.

Zusammengefasst kann formuliert werden, dass bei einer Fokussierung der Biografien der am Unterrichtsgespräch beteiligten Personen das Thema Prostitution zwar als bedeutsam für Jugendliche wahrgenommen, jedoch nicht in eine unterrichtliche Erarbeitung überführt wird. Ein sozialwissenschaftliches Deutungsmuster, bezogen auf ein kognitiv ausgerichtetes Informieren gerade unterschiedlicher Auffassungen zum Thema, scheint das Unterrichtsgespräch dagegen zu begünstigen. Damit liegt ein erster Baustein für eine Systematisierung schulischer Sexualerziehung vor. Unter sexualtheoretischen Gesichtspunkten sollte Schule bei diesem Thema eine primär auf kognitives Lernen bezogene Institution darstellen. Eine kognitiv-informierende Deutung ist entsprechend eher als professionell zu kennzeichnen, da durch dieses Vorgehen der Unterricht vor allem informative Aspekte anbietet. Eine diesbezügliche Aneignung stellt sich als Aufgabe der Lehrerausbildung dar, die wiederum theoretische Erkenntnisse über das Sprechen über Sexualität im Unterricht als Grundlage haben muss. Dafür ist es wichtig, dass gegenstandsorientierte empirische Erkenntnisse über professionstheoretische Bezugsprobleme identifiziert werden. Unter anderem sollte erforscht werden, 
welche Deutungsmuster welche konkreten professionsbezogenen Implikationen enthalten. Hier konnte in einem ersten Zugang gezeigt werden, dass Deutungsmusteranalysen ein vielversprechendes Analyseverfahren darstellen. Ein wichtiges Ziel im Sinne einer Professionalisierung von Lehrenden und einer professionellen Unterrichtsdurchführung scheint dann erreicht, wenn Lehrende auf die in den Interviews auftauchende Frage eine Antwort wissen: „Was sagt man als Lehrer dazu?“

Insgesamt betrachtet stellt sich der Status Quo von Deutungsmusteranalysen in professionsbezogenen Forschungen als heterogen dar. Dies wirkt sich unmittelbar auf die jeweils projekteigene Konkretion der mit der Analyse einhergehenden terminologischen Bestimmungen sowie forschungsbezogenen Vorgehensweisen aus. Es sollte insgesamt eine konsensuale Vereindeutigung zentraler Bezeichnungen sowie methodologischer Prämissen angestrebt werden. Ertragreich scheinen Deutungsmusteranalysen für die Professionsforschung, um Haltungen und Überzeugungen herauszuarbeiten, die elementar für das professionelle Handeln und den Umgang mit berufsspezifischen Herausforderungen sind. Dabei sollten besonders jene methodologischen Überlegungen weiterentwickelt werden, die sich von der Fallbestimmung im Sinne eines personenabhängigen Einzelfallverständnisses lösen. Bei Deutungsmusteranalysen sollten die Fälle vielmehr vor dem Horizont interessierender Bezugsprobleme bestimmt werden, welche als immer wieder auftauchende Sujets durch den thematischen Vergleich von vielen Interviews identifiziert werden können. Gleichzeitig sollte die Frage verfolgt werden, wie theoretisch-abstrakte Herausforderungen oder Probleme des professionellen Lehrerhandelns ihre konkrete Bearbeitung durch Lehrer_innen erfahren, um auch deduktive Analysen zu gewährleisten. Eine entsprechende Diskussion zur systematischen, makroperspektivischmethodologischen Bestimmung von Deutungsmusteranalysen könnte so die Möglichkeiten und Grenzen gegenüber den etablierten rekonstruktiv-qualitativen Analysen zur Professionsforschung um eine erkenntnisorientierte Forschungsmethode bereichern.

\section{Autorenangaben}

Dr. Markus Hoffmann

Universität zu Köln

Department Erziehungs- und Sozialwissenschaften

markus.hoffmann@uni-koeln.de

\section{Literaturverzeichnis}

Arnold, Rolf (1983): Deutungsmuster. Zu den Bedeutungselementen sowie den theoretischen und methodologischen Bezügen eines Begriffs. In: Zeitschrift für Pädagogik 29, 6, S. 893-912.

Bennewitz, Hedda (2008): Lehrende in Schulreformprozessen. Eine Deutungsmusteranalyse. In: Breidenstein, G./Schütze, F. (Hrsg.): Paradoxien in der Reform der Schule. Ergebnisse qualitativer Sozialforschung. Wiesbaden: VS-Verlag, S. 247-260.

Blumer, Herbert (1954): What is Wrong with Social Theory? In: American Sociological Review 19, 1, S. 3-10.

Bögelein, Nicole (2016): Deutungsmuster von Strafe. Eine strafsoziologische Untersuchung am Beispiel der Geldstrafe. Wiesbaden: Springer Fachmedien. 
BZgA (2010): Jugendsexualität 2010. Repräsentative Wiederholungsbefragung von 14- bis 17-Jährigen und ihren Eltern - aktueller Schwerpunkt Migration -; Ergebnisse der aktuellen Repräsentativbefragung. Köln: BZgA.

Hoffmann, Markus (2016): Schulische Sexualerziehung. Deutungsmuster von Lehrenden. Leverkusen: Barbara Budrich.

Kelle, Udo/Kluge, Susann (2010): Vom Einzelfall zum Typus. Fallvergleich und Fallkontrastierung in der qualitativen Sozialforschung. 2. Aufl. Wiesbaden: VS-Verlag.

Kunze, Katharina (2011): Professionalisierung als biographisches Projekt. Professionelle Deutungsmuster und biographische Ressourcen von Waldorflehrerinnen und -lehrern. Wiesbaden: VS-Verlag.

Lüders, Christian (1991): Deutungsmusteranalyse. Annäherungen an ein risikoreiches Konzept. In: Garz, D./Kraimer, K. (Hrsg.): Qualitativ-empirische Sozialforschung. Konzepte, Methoden, Analysen. Opladen: Westdeutscher Verlag, S. 377-408.

Meister, Gudrun (2005): Das unterrichtliche Selbstverständnis von LehrerInnen. Empirische Muster im Kontext von Unterricht und Biographie. Wiesbaden: VS-Verlag.

Oevermann, Ulrich (1973): Zur Analyse der Struktur von sozialen Deutungsmustern. Unveröffentlichtes Manuskript. [http://publikationen.ub.uni-frankfurt.de/frontdoor/deliver/ index/docId/4951/file/Struktur-von-Deutungsmuster-1973.pdf], Zugriff am 21.02.2017.

Oevermann, Ulrich (2001): Die Struktur sozialer Deutungsmuster - Versuch einer Aktualisierung. In: Sozialer Sinn 1, 1, S. 35-81.

Schmidt, Renate-Berenike (2013): Sexualerziehung in der Sekundarstufe I. In: Schmidt, R.-B./ Sielert, U. (Hrsg.): Handbuch Sexualpädagogik und sexuelle Bildung. 2. Aufl. Weinheim: Beltz Juventa, S. 593-600.

Ullrich, Carsten G. (1999): Deutungsmusteranalyse und diskursives Interview. In: Zeitschrift für Soziologie 28, 6, S. 429-447.

Weigelt, Linda (2008): Deutungsmuster von Sportlehrkräften zu Nähe und Distanz im Unterricht. Eine qualitative Studie. In: Oesterhelt, V./Hofmann, J./Schimanski, M./Scholz, M./ Altenberger, H. (Hrsg.): Sportpädagogik im Spannungsfeld gesellschaftlicher Erwartungen, wissenschaftlicher Ansprüche und empirischer Befunde. Schriften der Deutschen Vereinigung für Sportwissenschaft, S. 69-72.

Witzel, Andreas (1989): Das problemzentrierte Interview. In: Jüttemann, G. (Hrsg.): Qualitative Forschung in der Psychologie. Grundfragen, Verfahrensweisen, Anwendungsfelder. 2. Aufl. Heidelberg: Asanger, S. 227-256.

Witzel, Andreas/Reiter, Herwig (2012): The problem-centred interview. Principles and Practice. Los Angeles: Sage. 


\title{
Rezensionen
}

Till-Sebastian Idel

\author{
Alkemeyer, Thomas/Kalthoff, Herbert/Rieger-Ladich, Markus \\ (2015). Bildungspraxis. Körper, Räume, Objekte. \\ Weilerswist: Velbrück Wissenschaft. ISBN 978-3-95832-067-3. \\ 344 S. 29,90 Euro
}

In der pädagogischen Theorietradition wurde und wird Bildung klassischerweise stets als mehr oder weniger individueller Vorgang verstanden, in dem sich der Einzelne in mentalen Prozessen der Selbstentäußerung und des Rückbezugs auf sich in den Status reflexiver Subjektivität bringt. Die qualitative Bildungs-, Schul- und Unterrichtsforschung versteht diese Prozesse per definitionem als sozial kontextuiert und konstituiert. Allerdings ist es erst dem Forschungsprogramm einer Praxeografie des pädagogischen Felds gelungen, die Materialität von Bildungsprozessen theoretisch gehaltvoll und in einer mikroethnografisch feinkörnigen Beobachtung in den Blick zu bekommen und damit eine sozialtheoretische Fixierung auf das Mentale zu überschreiten. Der hier nur in aller Kürze anzuzeigende Band versammelt solche praxistheoretisch inspirierten Detailstudien, die Bildung - so die Herausgeber in der Einleitung - nicht als ,weitgehend kontextfreies, rein geistiges Geschehen eines körperlosen und eigentümlichen weltlosen Subjekts“ (S. 9), sondern als situierte und damit in ihren konkreten Vollzügen beobachtbare und beschreibbare soziale Praxis entwerfen. Praxistheoretisch verstanden werden wir zu Gebildeten gemacht und bilden uns, indem wir uns in soziale Praktiken einfädeln, die Räume als Örtlichkeiten entstehen lassen, uns mit wissensgeladenen Dingen konfrontieren und uns körperhafte Leiblichkeit verleihen. Dinge sind dann nicht einfach nur als Gegenstände da, Räume nicht einfach nur als Behälter vorhanden, Körper nicht einfach nur das auf reine Physis reduzierbare Substrat unseres Daseins und Bildung eben nicht nur die innere Selbstkonstitution des Geists.

Wie dieses Verständnis der Materialität von Praxisvollzügen, die soziale Arrangements und uns als signifizierte Subjekte hervorbringen und die wir in eigenen Handlungen im Rahmen von Praktiken fortschreiben, in unterschiedlichen Ansätzen der Familie der Praxistheorien von Goffman und Garfinkel über Bourdieu und Foucault bis hin zu Latour und Schatzki konzeptualisiert wird, erläutern die Herausgeber in der Einleitung, die das mittlerweile ausdifferenzierte praxeologische Theorietableau sachkundig beschreibt. Die folgenden Einzelbeiträge richten ihre analytische Aufmerksamkeit auf jeweils eine der drei genannten Dimensionen von Materialität, teilweise liegen sie auch an Schnittstellen, und leuchten somit jeweils für sich feinkörnig den Vollzug von pädagogisch institutionalisierten Bildungspraxen aus, vornehmlich im Feld der Schule. Alle Beiträge zeichnen sich durch eine fruchtbare Verbindung von theoretischer Sensitivität und empirischen Detailanalysen aus. Sie fragen etwa danach, wie mit dem Körper gelernt wird, körperliches Wissen entsteht bzw. Kompetenzen verkörpert werden, sie 
setzen sich mit der Aneignung und Nutzung des Raums in poetischen Raumpraktiken auseinander und sie erproben die methodologische und analytische Tragfähigkeit des in der Akteur-Netzwerk-Theorie Latours den Dingen zugewiesenen Akteursstatus. Dabei machen sie die Spannung zwischen den präfigurierenden Kräften der materiellen und sozialen Anordnungsverhältnisse innerhalb von Praxisvollzügen auf der einen Seite und den zugleich schöpferisch-mimetischen Modi des Teilnehmens der Akteure auf der anderen Seite deutlich. Während die Beiträge in ihrer Gesamtheit das mittlerweile erreichte Theorieniveau und die dokumentarische Qualität der mikroethnografischen Tiefenschärfe belegen, erscheinen insbesondere jene Beiträge, die entweder unmittelbar in oder im Schnittfeld zur Fachdidaktik ansetzen, besonders aufschlussreich für die interpretative Unterrichtsforschung zu sein. Der exemplarische Bezug der meisten dieser Beiträge auf den Mathematikunterricht markiert das Desiderat einer praxeologischen Forschung in der fachdidaktischen Vielfalt und Breite. ${ }^{1}$ Der Band insgesamt vermag aber ebenso der bildungstheoretischen Forschung innerhalb der Erziehungswissenschaft, die sich im Zuge ihrer Empirisierung sozial- und subjekttheoretisch auch praxeologisch fundiert, wichtige Impulse zu geben.

\section{Autorenangaben}

Till-Sebastian Idel Universität Bremen idel@uni-bremen.de

1 Vgl. auch die Beiträge zum Themenschwerpunkt des 2015 erschienen Heft 4 der ZISU: Dinge, Wissen, Fachkulturen: Materialitäten in Schule und Unterricht. 